\title{
Métodos matemáticos em teorias de calibre
}

\author{
Henrique de Andrade Gomes
}

\author{
Dissertação Apresentada \\ ao \\ Instituto de Matemática e Estatística \\ da \\ Universidade de São Paulo \\ para \\ Obtenção do Grau de Mestre \\ em \\ Ciências \\ Área de concentração: Matemática \\ Orientador: Prof. Dr. Claudio Gorodski
}

Durante a elaboração deste trabalho o autor recebeu auxílio financeiro da CAPES/CNPq.

São Paulo, outubro de 2006. 


\title{
Métodos matemáticos em teorias de calibre
}

\author{
Este exemplar corresponde à redação \\ final da tese devidamente corrigida \\ e defendida por Henrique de Andrade Gomes \\ e aprovada pela comissão julgadora.
}

São Paulo, outubro de 2006.

Banca examinadora:

- Prof. Dr. Marcos Alexandrino - IME-USP

- Prof. Dr. Frank Michael Forger -IME-USP

- Prof. Dr. Marcos Jardim - IME-UNICAMP 


\section{Agradecimentos}

Primeiramente, gostaria de agradecer encarecidamente ao meu orientador, Professor Claudio Gorodski, pelas ricas discussões e por ensinar-me tanto sobre como fazer matemática. Seu modo de pensar será sempre uma referência para mim.

Dedico esse trabalho aos meus pais, que sempre aturaram com paciência meus hábitos anormais, estimularam a busca pelo conhecimento, instigando em mim uma admiração infindável pela nobreza dessa empreitada, e por vezes até fingiram interesse em meus estudos.

Gostaria de agradecer também à minha companheira Helena, pelo seu estímulo aos meus hábitos anormais, paciência com minha busca pelo conhecimento, e por, vez em quando, também fingir interesse. 


\section{Resumo}

Neste trabalho esmiuçamos a interpretação geométrica de teorias de gauge através de fibrados. Conduzimos ainda uma investigação na área de topologia de fibrados, fornecendo inclusive uma demonstração do teorema de classificação de fibrados. Por último fornecemos algumas aplicações, como eletromagnetismo e teoria de Kaluza-Klein.

\section{Abstract}

In the present work we explore the geometrical interpretation of gauge theories through the formalism of fiber bundles. Moreover, we conduct an investigation in the topology of fiber bundles, providing a proof of the Classification Theorem. In the last chapter we present some applications, such as electromagnetism and generalized Kaluza-Klein Theory. 


\section{Palavras-chave}

Fibrado vetoriais, fibrados principais, conexões, gauge, Kaluza-Klein, classificação de fibrados.

\section{Key words}

Vector bundles, principal fiber bundles, connections, Kaluza-Klein, classification of vector bundles. 


\section{Sumário}

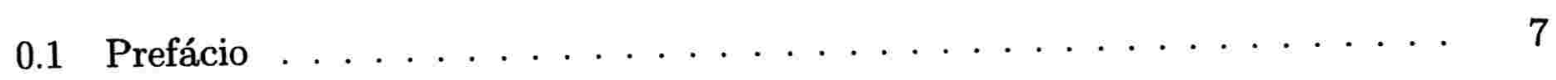

0.2 Introdução . . . . . . . . . . . . . . . . . 7

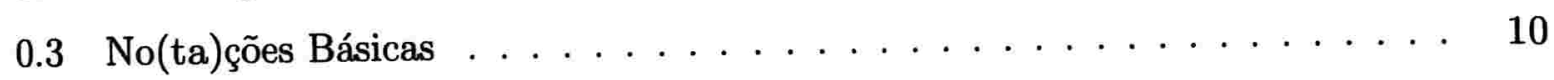

1 Fibrados Vetoriais 12

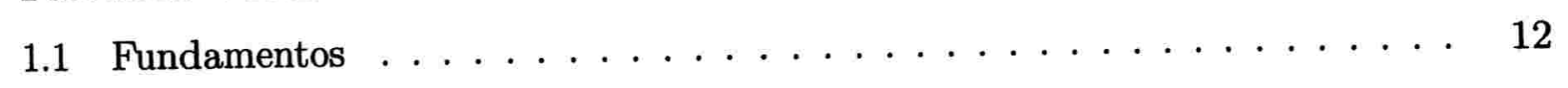

1.2 Operadores Diferenciais Lineares $\ldots \ldots \ldots \ldots \ldots$

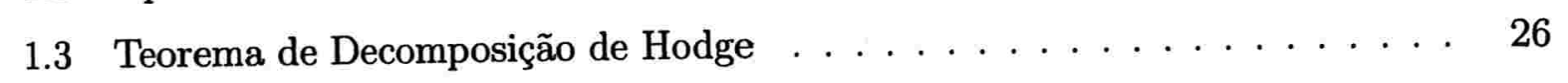

1.4 Conexões em Fibrados Vetoriais . . . . . . . . . . . . . . . . . 33

1.5 Transporte Paralelo e Holonomia . . . . . . . . . . . . . . . . . . . . . . . . . . . . . .

1.6 Conexões Admissíveis e Derivada de Gauge . . . . . . . . . . . . . . . . . . 49

2 Fibrados Principais $\quad \mathbf{5 7}$

2.1 Fundamentos . . . . . . . . . . . . . . . . 57

2.2 Conexões em Fibrados Principais . . . . . . . . . . . . . 66

2.3 Fibrado dos Referenciais . . . . . . . . . . . . . . 75

2.4 Fibrados Associados . . . . . . . . . . . . . . . 77

3 Campos de Yang-Mills e Classes Características 82

3.1 Yang-Mills . . . . . . . . . . . . . . . . . 82

3.2 Classificação de Fibrados . . . . . . . . . . . . . . . . . . 92

4 Aplicações 103

4.1 Eletromagnetismo . . . . . . . . . . . . . . . . . 103

4.2 Kaluza-Klein . . . . . . . . . . . . . . . . 108 


\subsection{Prefácio}

Na matemática, um manipulador formal frequentemente experiencia a desconfortável sensação de que seu lápis é mais inteligente do que ele. - Howard Eves

Esta dissertação é baseada em [7] - notas de um curso ministrado por Richard Palais em 1981 entitulado Geometrização da Física. Este curso, dado no curto período de seis semanas, está em forma bastante resumida, com demonstrações muitas vezes omitidas ou brevemente "indicadas".

Algumas das demonstrações contidas no presente trabalho são mais "sujas" do que as encontradas nos livros textos usuais, por terem sido elaboradas independentemente, com a ajuda de meu orientador, ou ainda com os norteamentos gerais das notas do Palais. Peço paciência aos leitores se lhes parecerem óbvias demonstrações alternativas.

Em todo momento a exposição é acompanhada por um subtexto interpretativo, numa tentativa de compreender em um nível mais intuitivo e geométrico os resultados a que chegamos. Tomei esta atitude unicamente em benefício próprio, com a esperança de permanecer "mais inteligente do que meu lápis". Nessa empreitada sigo o espírito de [8], e [4]. Preferi essa atitude a destinar minha dissertação unicamente aos meus examinadores, que por seu conhecimento profundo do assunto têm provavelmente pouco a ganhar com uma exposição menos seca.

O objetivo deste estudo foi o de me introduzir ao vasto arcabouço matemático necessário ao físico teórico moderno, um horizonte um pouco mais difuso do que a dissertação de mestrado usual (baseada em um teorema ou artigo), e responsável pela aparente desconexão de alguns tópicos, como o Teorema de Hodge, que, apesar de sua enorme importância, não é reutilizado no decorrer da exposição.

Primeiramente, gostaria de agradecer encarecidamente ao meu orientador, Professor Claudio Gorodski, pelas ricas discussões e por ensinar-me tanto sobre como fazer matemática. Seu modo de pensar será sempre uma referência para mim.

Dedico esse trabalho aos meus pais, que sempre aturaram com paciência meus hábitos anormais, estimularam a busca pelo conhecimento, instigando em mim uma admiração infindável pela nobreza dessa empreitada, e por vezes até fingiram interesse em meus estudos.

Gostaria de agradecer também à minha companheira Helena, pelo seu estímulo aos meus hábitos anormais, paciência com minha busca pelo conhecimento, e por, vez em quando, também fingir interesse.

\subsection{Introdução}

A Filosofia é escrita neste grande livro - o Universo - que permanece sempre aberto ao nosso mirar, mas que não pode ser compreendido a não ser que primeiro se aprenda a compreender a linguagem e interpretar os caractéres em que é escrito. Ele é escrito na linguagem da matemática, e seus caractéres são triângulos, círculos, e outras figuras geométricas, sem as quais é humanamente impossivel compreender dele uma só palavra.- Galileo Galilei 
A relação íntima entre geometria e física, apesar de bem explicitada pela citação acima, não começou com Galileo e certamente não se extinguiu desde então. Deveras, desde Pitágoras, Euclides e Arquimedes se busca refletir a irregular realidade no claro espelho da geometria.

Ao tornarmos o olhar para o desenvolvimento científico atual, podemos dizer com segurança que a teoria da Relatividade Geral foi um dos maiores desenvolvimentos da ciência no século XX, um capítulo importantíssimo no grande livro de Galileo, um espelho geométrico límpido que inspirou inúmeras ramificações na física e na matemática. Na mecânica Newtoniana, o espaço e o tempo eram encarados como palcos fixos, onde tinha lugar a dinâmica do Universo conhecido. Estes palcos no entanto, permaneciam alheios, indiferentes ao que neles se desenrolava. Matematicamente, o espaço nesta teoria seria representado por uma cópia de $\mathbb{R}^{3}$ e o tempo por uma cópia de $\mathbb{R}$, i.e.: $\mathbb{R}^{3} \times \mathbb{R}$, ambos fixos e imiscíveis, .

Com o advento da teoria eletromagnética da Maxwell, foi necessária uma revisão destes conceitos. Maxwell mostrou que duas teorias aparentemente distintas, a elétrica e a magnética, eram simplesmente dois aspectos do "campo eletromagnético". Explicou assim a luz como um fenômeno decorrente de distúrbios no campo elétrico gerando distúrbios no campo magnético e vice-versa, criando um mecanismo retroativo, propagando-se no espaço e no tempo. 'Acidentalmente', essa forma de propagação deveria ter uma velocidade fixa, o mecanismo deveria "girar" sempre à mesma velocidade, o que significava que a luz emitida por um corpo seria percebida com a mesma velocidade por todos os observadores, irrespectivamente de seu movimento em relação à fonte.

Isso levou Lorentz, Poincaré e especialmente Einstein, a perceberem que para acomodar a teoria de Maxwell, o espaço e o tempo não poderiam permanecer imiscíveis. Assim originouse a revolução da relatividade restrita, que transformou a nossa visão do espaço e do tempo, fundindo os dois em um só, o espaço-tempo. De $\mathbb{R}^{3} \times \mathbb{R}$, passamos a enxergar o palco da realidade como $\mathbb{H}^{4}$, o espaço de Minkowski quadri-dimensional ${ }^{1}$.

Einstein ao tentar expandir essa teoria para que englobasse também a gravidade, naturalmente tentou seguir o modelo de Maxwell, porém foi forçado a construir uma teoria em que o espaço-tempo não poderiam mais assistir ao desenrolar dos eventos placidamente, mas deveriam ser afetados e afetar a dinâmica dos corpos de uma maneira fundamental. Grosseiramente, as equações de Einstein ${ }^{2}$ dizem que energia e momento afetam a forma como medimos distância e duração no espaço-tempo assim como cargas e correntes afetam o campo eletromagnético. $O$ espaço-tempo deixou de ser globalmente identificado a $\mathbb{H}^{4} \mathrm{e}$ passou a ser localmente identificado a pedaços de $\mathbb{H}^{4}$, a ser encarado como uma variedade Lorentziana quadri-dimensional, onde as trajetórias naturais de partículas ${ }^{3}$ seriam representadas por geodésicas. Isso alimentou as esperanças de que talvez grande parte ou até mesmo toda a física tivesse caráter puramente geométrico, e fertilizou o solo para a invenção das teorias de gauge.

Em física, as chamadas teorias de gauge (ou calibre) são baseadas na idéia de que certas

${ }^{1} \mathrm{O}$ espaço plano de assinatura +++-

$$
R_{\mu \nu}-\frac{1}{2} g_{\mu \nu} R=\kappa T_{\mu \nu}
$$

onde $R_{\mu \nu}$ é o tensor de Ricci, $R$ é o escalar de curvatura e $g_{\mu \nu}$ é a métrica do espaço -tempo, $\kappa$ é uma constante, todos os termos serão definidos apropriadamente mais adiante.

${ }^{3}$ Partículas sem carga, cor, etc. 
transformações de simetria de um sistema podem ser efetuadas tanto local, como (às vezes) globalmente, sem afetar os resultados da teoria, i.e.: que a teoria é invariante por certas transformações. Estas simetrias localmente sempre refletem uma redundância na descrição de um sistema, o que é encarada como uma transformação "passiva", ou de coordenadas, enquanto que globalmente também podem ser relacionadas com transformações " de fato", ou ativas. Como veremos, a teoria eletromagnetica de Maxwell pode ser considerada o caso mais simples das chamadades teorias de gauge, seu exemplo "par excellence". Antecipando um pouco nossa exposição, podemos perceber que a definição de "terra" do potencial elétrico é um exemplo de simetria de gauge: o valor absoluto do potencial é imaterial, o que importa em qualquer sistema elétrico é a diferença entre potenciais. Esse é um caso de uma transformação global, se considerarmos também mudanças do potencial magnético, é possivel fazer mudanças locais sem afetar qualquer resultado.

A importância desta simetria no entanto permaneceu despercebida até uma tentativa de Hermann Weyl [1] de unificar eletromagnetismo e relatividade geral. Inspirado na simetria conforme das teorias de Maxwell, Weyl procurou interpretar o eletromagnetismo como uma distorção de comprimentos relativísticos produzidos pelo deslocamento sobre uma curva fechada. Conjecturou que invariância por mudanças em escala (ou calibre) poderiam também ser uma simetria local da relatividade geral. Superficialmente, seu raciocínio foi de que escala local não deveria ser observável, já que um aumento das dimensões de todos os objetos ao nosso redor não poderia ser detectável ${ }^{4}$. Contudo, formalmente chegou ao resultado de que o espectro de radiação dos átomos dependeria de sua história, um resultado não encontrado na Natureza [2], o que foi apontado por Einstein.

No entanto, após o desenvolvimento da mecânica quântica e suas funções complexas, tornou-se claro que fase, e não escala, era o que deveria construir a ponte com eltromagnetismo ${ }^{5}$. Weyl, Vladimir Fock e Fritz London reutilizaram a idéia inicial, substituindo o fator de escala por uma variável complexa, transformando a mudança de escala em uma mudança de fase (uma simetria de gauge $U(1)$, como veremos mais tarde). Alcançaram assim uma bela explicação para o efeito do campo eletromagnético sobre a descrição quântica de uma partícula carregada ${ }^{6}$. Infelizmente, nessa nova encarnação não havia mais uma maneira de incorporá-la na relatividade geral, e ela tinha de ser superposta como estrutura adicional sobre o espaço-tempo. Nascia assim a teoria de gauge.

Em 1954, na tentativa de resolver problemas na teoria de partículas elementares, Chen Ning Yang e Robert Mills introduziram teorias de gauge com grupos de simetria não abelianos como modelos para a interação forte; a cola que permite a coesão dos núcleos atômicos. No entanto sua ligação com a teoria de fibrados permaneceu largamente ignorada ou considerada irrelevante até os anos 70 , quando aspectos não perturbativos relacionados às soluções clássicas das equações de Yang-Mills (instantons) vieram à tona, incorporando questões globais da teoria de fibrados.

Paradoxalmente, o formalismo matemático das teorias de gauge proveram uma estrutura para a unificação das teorias quânticas de campos, notavelmente incompatíveis com a rela-

\footnotetext{
${ }^{4}$ Matematicamente, ele postulou um transporte paralelo alternativo, que não preservava a norma.

${ }^{5} \mathrm{Ou}$ em linguagem moderna, o grupo de Gauge deveria ser modelado em $S^{1}$ ao invés do grupo multipilcativo $\mathbb{R}$.

${ }^{6}$ Assunto que não abordaremos nessa dissertação, já que nos ateremos às formulações clássicas ( i.e.: não quânticas) da teoria.
} 
tividade geral, um dos conceitos progenitores da teoria de gauge. O Modelo Padrão descreve com alto grau de precisão as interações fraca, forte e eletromagnética, através de um grupo de simetrias não-abeliano $S U(3) \times S U(2) \times U(1)$.

Apesar da motivação inicial destas teorias terem sido de ordem física, assim como em praticamente todas as outras construções relevantes do campo, a interação e benesse recíproca entre física e matemática provinda das teorias de gauge foi extremamente fértil. Aos anos 70, Michael Atiyah, estudou as soluções das equações clássicas de Yang-Mills, e em 1983, Donaldson, aluno de Atiyah, utilizando este trabalho revolucionou o estudo de variedades de dimensão 4. Michael Freedman, continuando esse estudo, conseguiu exibir estruturas diferenciáveis "fake" do $\mathbb{R}^{4}$ ( i.e.: diferentes da canônica). Isso levou a um grande interesse em teorias de gauge por seus resultados puramente matemáticos. Em 1994, Edward Witten e Nathan Seiberg, inventaram métodos de calcular invariantes topológicos baseados em teorias de gauge.

Obviamente, nosso âmbito nessa dissertação é bem mais modesto. Pretendemos fornecer somente um apanhado inicial, e pessoal, da teoria de gauge clássica, com ênfase no aspecto geométrico. O presente trabalho é uma tentativa de apresentar as ferramentas matemáticas necessárias a essa empreitada; à geometrização da física. As chamadas teorias de gauge (ou de calibre) conseguem uma representação puramente geométrica das interações não gravitacionais, utilizando intensamente o maquinário matemático da teoria de fibrados. Vamos a ela.

\subsection{No(ta)ções Básicas}

\section{Por essa razão é seu nome Babel; porque o Senhor ali confundiu a linguagem de toda a Terra.- Genesis 11;9}

Chamaremos normalmente a variedade espaço-tempo de $M$, mas faremos poucas referências ao seu caráter Lorentziano. O tratamento de teorias físicas modelando o espaçotempo através de variedades diferenciáveis automaticamente implementa um dos maiores insights provindos da relatividade geral: a invariância das leis físicas por mudanças de coordenadas ${ }^{7}$.

Para facilitar a notação, salvo aviso, utilzaremos a convenção de soma de Einstein, pela qual se soma índices repetidos em cima e em baixo, e.g.:

$$
A^{i} B_{i}=\sum_{i} A^{i} B_{i}
$$

Aproveitamos o ensejo para apontar que se $A, B$ são matrizes $k \times k$ nós temos que $[A B]_{j}^{i}=$ $A_{k}^{i} B_{j}^{k}=B_{j}^{k} A_{k}^{i} \neq[B A]_{j}^{i}=B_{k}^{i} A_{j}^{k}$ i.e.: para passarmos da notação escalar para a matricial precisamos arranjar a ordem correta, pré estabelecida dos termos.

Ainda no tópico "índices", utilizaremos para bases de $p$-formas muitas vezes a notação de multi-índices, normalmente denotados por $I$ e $J$, que não devem ser confundidos com os índices latinos maíusculos $A$ e $B$ que utilizaremos na seção sobre a teoria de Kaluza-Klein,

\footnotetext{
${ }^{7} \mathrm{E}$ sua invariância por difeomorfismos globais, que sucitou algumas discussões interessantes em relação ao " argumento do buraco" (hole argument) [15].
} 
onde denotam índices normais que percorrem os índices usuais sobre $M$ e os índices do espaço interno.

Buscando satisfazer o físico que há em todos nós, bem como ilustrar um método bastante útil em cálculos explícitos, tentaremos alternar demonstrações usando bases locais com demonstrações mais intrínsecas, sem índices.

Assumiremos um conhecimento básico de variedades diferenciáveis, campos vetoriais e grupos de Lie, o referente a um subconjunto denso dos primeiros três capítulos de [6]. Em especial mencionamos os dois seguintes teoremas:

Theorem 1 Sejam $M, N$ variedades suaves de dimensões $m$ e $n$ respectivamente e $f: M \rightarrow$ $N$ suave. Se $Q$ é subvariedade mergulhada em $N$, então $S=f^{-1}(Q)$ é subvariedade mergulhada de $M$ se e somente se para todo $p \in S$ nós tivermos $T_{f(p)} N=\operatorname{Im}\left(d f_{p}\right)+T_{f(p)} Q$.

Há uma bela e simples demonstração deste teorema em [17].

Um outro teorema que nos servirá em diversas discussões é o de Frobenius.

Theorem 2 Uma k-distribuição suave involutiva em uma variedade $M^{m}, m \geq k$, é integrável.

Onde uma $k$-distribuição em uma variedade $M$ é um mapa $\mathfrak{D}: M \rightarrow T M$ que assinala a cada ponto $m \in M$ um subespaço $k$-dimensional de $T_{m} M, \mathfrak{D}_{m}$. A $k$-distribuição é dita suave se cada ponto $m \in M$ tem vizinhança aberta $U$ onde $\mathfrak{D}$ é gerado por $k$ campos suaves em $U$ linearmente independentes. Uma variedade integral de $\mathfrak{D}$ é uma subvariedade $N^{k}$ de $M$ tal que $T_{m} N=\mathfrak{D}_{m}$ para todo $m \in N$. $\mathfrak{D}$ é dita involutiva se para todos os campos suaves $X, Y \in \mathfrak{D}$, nós tivermos $[X, Y] \in \mathfrak{D}$. As demonstrações geométricas são padrão, procedendo por indução a partir do Teorema do Fluxo Tubular, ver [3]. 


\section{Chapter 1}

\section{Fibrados Vetoriais}

\section{$1.1 \quad$ Fundamentos}

As equações de Maxwell e os princípios da mecânica quântica levaram à idéia de invariância de gauge. Tentativas de generalizar essa idéia, motivada pelos conceitos físicos de fases, simetrias e leis de conservação, levaram à teoria de gauge de campos não abelianos. Que campos de gauge não abelianos são conceitualmente idênticos a idéias na bela teoria de fibrados, desenvolvida por matemáticos sem referência ao mundo físico, deixava-me estupefato. Em 1975, discuti minhas considerações con Chern, $e$ disse "isso é tanto emocionante quanto desconcertante, já que vocês matemáticos inventaram esses conceitos do nada." Imediatamente ele protestou: "Não, não. Estes conceitos não foram sonhados. Eles foram naturais e reais."- C. N. Yang

\section{Fibrados}

Ao medirmos qualquer quantidade física, a estamos medindo localmente, i.e.: sobre um aberto de $M$. Podemos dizer que essa quantidade mora em um espaço " interno" $E_{x}$ sobre cada ponto $x$ do espaço-tempo, e sua medição implica uma projeção sobre $M$. A forma mais simples de "campo" seria assim uma função $f: M \rightarrow E$, supondo que o campo sobre cada ponto " mora" no mesmo espaço, $E \simeq E_{x}$. No entanto, tal asserção implica que podemos comparar valores do campo sobre toda variedade $M$, Por outro lado, não necessariamente possuímos um sistema de coordenadas global, no qual descreveríamos qualquer quantidade física, então parece precipitado instituir a priori que podemos descrever o campo globalmente. A priori, nós temos apenas uma união disjunta dos espaços internos sobre cada ponto, um "feixe" de espaços internos ${ }^{1}$.

Definition 1 Um fibrado consiste de variedades $E$ (chamado de espaço total), e $M$ (espaço base) e um mapa diferenciável sobrejetor $\pi: E \rightarrow M$ (projeção). $E_{x}=\pi^{-1}(x)$ é chamada de fibra sobre $x$.

\footnotetext{
${ }^{1}$ Essa seria a tradução mais adequada para "bundle", infelizmente já é utilizada em outra área da matemática.
} 
Como a projeção é sobrejetora, é claro que $\bigcup_{x \in M} E_{x}=E$. Em geral, as fibras $E_{x}$ não precisariam ser isomorfas mas esse é o caso interessante para nós, por isso assumiremos essa condição, i.e.: $E_{x} \simeq E_{x^{\prime}}$ para todos $x, x^{\prime} \in M$. Nós estamos então tomando uma única variedade para a descrição das fibras e não uma coleção de variedades, uma sobre cada ponto. Chamamos um fibrado de localmente trivial se tivermos uma cobertura de $M,\left\{U_{\alpha}, \alpha \in \Lambda\right\}$, onde $\bigcup_{\alpha \in \Lambda} U_{\alpha}=M$, e para cada $U_{\alpha}$ exista $\psi_{\alpha}$ difeomorfismo tal que:

$$
\psi_{\alpha}: \pi^{-1}\left(U_{\alpha}\right) \longrightarrow U_{\alpha} \times F
$$

onde $F$ é uma variedade diferenciável fixada para todo $\alpha$, chamada de fibra típica de $E$. Agora, nós queremos também que

$$
\begin{aligned}
\psi_{\alpha}: & \pi^{-1}(x) \rightarrow\{x\} \times F \\
& u \longmapsto\left(x, \psi_{\alpha, x}(u)\right)
\end{aligned}
$$

onde, como $\psi$ é difeomorfismo, $\psi_{\alpha, x}: \pi^{-1}(x) \rightarrow F$ é também difeomorfismo. É importante notar que a equação (1.1) por si só não implica em (1.2), já que se $\tilde{U}_{\alpha} \subset U_{\alpha}$, não é necessariamente verdade que a restrição de $\psi$ seja da seguinte forma:

$$
\psi_{\alpha}: \pi^{-1}\left(\tilde{U}_{\alpha}\right) \longrightarrow \tilde{U}_{\alpha} \times F
$$

Essa condição, i.e.: (1.3) para todo aberto $\tilde{U}_{\alpha} \subset U_{\alpha}$, equivale a (1.1). Essa caracterização sobre a trivialização é imprescindível para que ela mantenha o caráter de fibras sobre os pontos do fibrado. Em outras palavras, impomos na definição que $p r_{1} \circ \psi=\pi$, onde $p r_{1}$ é a projeção na primeira coordenada.

Ou seja, um fibrado localmente trivial (ao qual nos referiremos mais adiante simplesmente como fibrado) é simplesmente uma variedade que localmente é uma variedade produto ${ }^{2}$.

\section{EXEMPLOS:}

\section{- O fibrado trivial}

$E=M \times N$. Esse é o caso mais simples de fibrado, chamado de trivial. A projeção é simplesmente a projeção na primeira coordenada, i.e.:

$$
\begin{array}{r}
\pi: E \rightarrow M \\
(x, v) \mapsto x
\end{array}
$$

Este fibrado é claramente localmente trivial, pela restrição da primeira coordenada.

- Faixa de Moebius

Seja $\sigma:\{I \times \mathbb{R}\} \rightarrow\{I \times \mathbb{R}\} / \sim=E$ onde a relação de equivalência é dada por $(0, t) \sim(1,-t)$. Então $\pi: I \times \mathbb{R} \rightarrow I$ induz um mapa $\tilde{\pi}: E \rightarrow S^{1}$ e a fibra aqui é $\mathbb{R}$, e E seria homeomorfo à faixa de Möbius sem as fronteiras. Com essa fibração, a faixa de Moebius é um exemplo de um fibrado localmente trivial (basta vermos que $\sigma:] 0,1[\times \mathbb{R} \rightarrow E$ é uma trivialização local), porém não trivial, i.e.: não é uma variedade produto. Isso é facilmente observável já que $S^{\mathbf{1}} \times \mathbb{R}$ é um cilindro, logo orientável e portanto não pode ser difeomorfo à faixa de Moebius.

\footnotetext{
${ }^{2}$ Veremos daqui a pouco porque variedades localmente produtos são mais interessantes para nós.
} 


\section{- O fibrado tangente TM}

$T M=\bigcup_{x \in M} T_{x} M=\left\{(x, v), x \in M, v \in T_{x} M\right\}$ então

$$
\begin{aligned}
\pi: T M & \rightarrow M \\
(x, v) & \mapsto x
\end{aligned}
$$

i.e.: se $w \in T_{x} M$ então $\pi(w)=x$, dada um atlas para $M,\left\{U_{\alpha}, \psi_{\alpha}\right\}$, nós temos que $T M$ é variedade diferenciável com atlas dado por

$$
\begin{aligned}
\left(\psi_{\alpha} \circ \pi, d \psi_{\alpha}\right): \pi^{-1}\left(U_{\alpha}\right) & \rightarrow W \times \mathbb{R}^{n} \subset \mathbb{R}^{n} \times \mathbb{R}^{n} \\
(x, v) & \mapsto\left(\psi_{\alpha}(x),\left(d \psi_{\alpha}\right)_{x}(v)\right)
\end{aligned}
$$

Aqui a fibra típica é isomorfa à $\mathbb{R}^{n}$ e uma trivialização local pode ser dada como acima, substituindo $\psi_{\alpha} \circ \pi \rightarrow \pi$. É ainda fá cil vermos que como as cartas são compatíveis, também os serão as trivializações locais.

Uma primeira questão que pode surgir naturalmente é se a condição de trivialidade local não é um corloário das outras propriedades de fibrados. I.e.: se $E$ é diferenciável, e as fibras são isomorfas, não seri razoável que $E$ fosse localmente uma variedade produto? A resposta é negativa, mostremos um contra-exemplo baseado na faixa de Moebius "torta".

Como vimos, tomada como fibrado sobre a base $S^{1}$ com fibra típica dada por um segmento de reta, a faixa de Moebius é um exemplo de um fibrado não trivial. Mas partindo da construção acima com $\{I \times \mathbb{R}\} / \sim$ podemos

podemos escolher tomar como variedade base, ao invés de $S^{1}$ (ou melhor, $I \times\{0\} / \sim$ ), uma semi-reta perpendicular a $S^{1}$, por exemplo

$$
\{0\} \times[0, \infty[=\{0\} \times \mathbb{R} / \sim
$$

as fibras então serão as projeções:

$$
\pi^{-\mathrm{i}}(a)=\sigma(I \times\{a\}), a \in[0, \infty[
$$

ou seja, variedades compactas unidimensionais. Logo, a fibra típica será dada por $S^{1}$. Mas agora, trivializações locais deveriam ser difeomorfos a $J \times S^{\mathbf{1}}$, onde $J$ é um intervalo de $[0, \infty[$. Ou seja, deveriam ser segmentos de cilindros. Mas é fácil ver que para qualquer intervalo aberto $W \in \mathbb{R}$ que contenha a origem, $\sigma(I \times W)$ é uma faixa de Moebius, portanto não orientável, portanto não difeomorfa a um cilindro.

Definition 2 Um fibrado vetorial é um fibrado localmente trivial, cujas fibras são espaços vetoriais, $e$ tais que, para todo $x \in M, \phi_{x}$ definido em (1.2) é isomorfismo linear.

Chamamos de $n$-fibrado vetorial (real) se a dimensão da fibra típica é $n$. Ou seja, nós temos que

$$
\psi: \pi^{-1}(x) \rightarrow\{x\} \times \mathbb{R}^{n}
$$

é um isomorfismo linear para todo $x \in M$. Poderíamos tomar da mesma forma $\mathbb{C}^{n}$ ao invés de $\mathbb{R}^{n}$. 
Definimos um isomorfismo entre fibrados vetoriais sobre uma mesma base $M$ (ou seja, entre as triplas previamente definidas $\left.\left(E_{1}, \pi_{1}, M\right),\left(E_{2}, \pi_{2}, M\right)\right)$, como um difeomorfismo $f: E_{1} \rightarrow E_{2}$ tal que $\pi \circ f=\pi$, onde $f$ leva $\pi_{1}^{-1}(x) \rightarrow \pi_{2}^{-1}(x)$ por um isomorfismo linear. Analogamente, um morfismo entre $\left.\left(E_{1}, \pi_{1}, M\right),\left(E_{2}, \pi_{2}, M\right)\right)$ é uma função suave $f: E_{1} \rightarrow E_{2}$ tal que $\pi \circ f=\pi$, onde $f$ leva $\pi_{1}^{-1}(x) \rightarrow \pi_{2}^{-1}(x)$ linearmente.

Definition 3 Uma seção de um fibrado vetorial $(E, \pi, M)$ é simplesmente uma função $s$ : $M \rightarrow E$, tal que para cada $x \in M s(x) \in \pi^{-1}(x)$.

Assim uma seção suave sobre o domínio de uma trivialização local $\theta$, pode ser identificado com uma função suave sobre a fibra típica, i.e.:, para todo $x \in \theta s: x \mapsto\left(x, f_{s}(x)\right)$.

Isso nos permite vizualizar concretamente o motivo principal para que tomemos o fibrado como sendo localmente trivial: é nele possível tomar seções suaves como sendo localmente expressas por func cões suaves a valores nas fibras típicas. Para se convencer de que isso não é possível em fibrados que não são localmente triviais, construa uma seção na faixa de Moebius "torta" e uma função $f:[0, b] \rightarrow S^{1}$ que a represente. Verifique que $f$ é descontínua na origem.

Denotaremos por $\Gamma\left(E_{\mid \theta}\right)$ o espaço de todas as seções sobre $\theta \subset M . s=s_{1}, \cdots, s_{k} \in \Gamma\left(E_{\mid \theta}\right)$ é chamado de base local de seções de $E$ sobre $\theta$ se a parametrização:

$$
\begin{array}{r}
\psi_{\theta}^{-1}: \theta \times \mathbb{R}^{k} \rightarrow \pi^{-1}(\theta)=E_{\mid \theta} \\
\left(p, \alpha_{1}, \cdots, \alpha_{k}\right) \longmapsto \alpha_{1} e_{1}(p)+\cdots+\alpha_{k} e_{k}(p)
\end{array}
$$

é um difeomorfismo, isto é: se as seções $e_{1}, \cdots, e_{k}$ são l.i. em cada ponto. É claro que $e_{i}(p)=\psi_{\theta}^{-1}\left(p, \tilde{e}_{i}\right)$, onde $\tilde{e}_{i}$ denota o i-ésimo vetor vetor da base canônica de $\mathbb{R}^{k}$. Então, localmente, dada uma base, uma seção pode ser expressa de forma unívoca por combinação $C^{\infty}(M)$-linearmente em termos dessa base.

Os mapas $F^{\theta}=\psi_{\theta}^{-1}$ são chamados de gauges locais de $\mathrm{E}$ sobre $\theta$. Suponhamos que temos dois gauges: $F^{\theta}=\psi_{\theta}^{-1}, F^{\beta}=\psi_{\beta}^{-1}$, então já que

$$
\begin{array}{r}
\psi_{\beta}^{-1}: x \times\left\{\mathbb{R}^{n}\right\} \rightarrow \pi^{-1}(x) \\
\psi_{\theta}: \pi^{-1}(x) \rightarrow x \times\left\{\mathbb{R}^{n}\right\}
\end{array}
$$

são ambos isomorfismos lineares, $\psi_{\theta} \circ \psi_{\beta}^{-1}: x \times\left\{\mathbb{R}^{n}\right\} \rightarrow x \times\left\{\mathbb{R}^{n}\right\}$ é isomorfismo linear. Logo nós temos

$$
\begin{aligned}
& \psi_{\theta} \circ \psi_{\beta}{ }^{-1}: \theta \cap \beta \times \mathbb{R}^{k} \longrightarrow \theta \cap \beta \times \mathbb{R}^{k} \\
& (x, v) \longmapsto\left(x, g_{\theta \beta}(x) v\right)
\end{aligned}
$$

Onde, para cada $x \in \theta \cap \beta$, nós temos $g_{\theta \beta}(x)=\left(F_{x}^{\theta}\right)^{-1} \circ F_{x}^{\beta}: \mathbb{R}^{k} \rightarrow \mathbb{R}^{k}$ é isomorfismo linear, isto é: $g_{\theta \beta}: \theta \cap \beta \rightarrow G L(k)$ é chamado de mapa de transição de gauge. É importante notar que apesar de uma fibra sobre um dado ponto ser isomorfa à $\mathbb{R}^{k}$, não existe isomorfismo canônico, qualquer trivialização local deve ser tão boa quanto outra, qualquer gauge é igualmente apropriado.

Por consistência devemos ter $g_{\alpha \alpha}=I d$. Agora, aplicando $\psi_{\beta} \circ \psi_{\theta}^{-1}$ de ambos os lados de (1.4), nós temos

$$
(x, v)=\psi_{\beta} \circ \psi_{\theta}^{-1}\left(x, g_{\theta \beta}(x) v\right)=\left(x, g_{\beta \theta} g_{\theta \beta}(x) v\right)
$$


Então

$$
g_{\beta \theta} g_{\theta \beta}=1
$$

Como essa equação é válida pra todo $x \in M$ e $g_{\theta \beta}(x) \in G L(k)$, nós temos que

$$
g_{\theta \beta}^{-1}=g_{\beta \theta}
$$

Além disso é claro que estamos identificando os pontos em $E$

$$
\psi_{\beta}^{-1}(x, v)=\psi_{\theta}^{-1}\left(x, g_{\theta \beta}(x) v\right)
$$

Logo

$$
\psi_{\gamma} \circ \psi_{\beta}^{-1}(x, v)=\left(x, g_{\gamma \beta}(x) v\right)=\psi_{\gamma} \circ \psi_{\theta}^{-1}\left(x, g_{\theta \beta}(x) v\right)=\left(x, g_{\gamma \theta} g_{\theta \beta}(x) v\right)
$$

Então

$$
g_{\gamma \beta}(x)=g_{\gamma \theta} g_{\theta \beta}(x), \forall x \in U_{\theta} \cap U_{\beta} \cap U_{\gamma}
$$

de onde , utilizando a equação (1.85), tiramos a condição de cociclo:

$$
g_{\gamma \theta} g_{\theta \beta} g_{\beta \gamma}=1
$$

Se, para todo ponto $x \in M$, e quaisquer trivializações $\theta$ e $\beta$, nós tivermos que a imagem de $g_{\theta \beta}$ está contida em um subgrupo $G \subset \mathbf{G L}(k)$, dizemos que $E$ é um $G$-fibrado vetorial sobre $M$. Generalizando, se tivermos um automorfismo local $T \in \Gamma\left(\operatorname{Aut}\left(\left.E\right|_{\beta}\right)\right)$, tal que para uma trivialização qualquer $\psi_{\beta}$ sobre $\beta$, nós tivermos

$$
\psi_{\beta} \circ T\left(\psi_{\beta}^{-1}(x, v)\right)=\left(x, g_{T}(x) v\right)
$$

onde a imagem de $g_{T}$ esteja contida em $G \subset \mathbf{G L}(k)$, chamaremos $T$ de transformação de gauge local. É trivial ver que se (1.11) vale para uma trivialização local sobre $\beta$, valerá também para qualquer outra $G$-trivialização sobre $\beta$ :

$$
T\left(\psi_{\beta}^{-1}(x, v)\right)=T\left(\psi_{\beta}^{\prime-1}\left(x, g_{\beta^{\prime} \beta}(x) v\right)\right)=\psi_{\beta}^{-1}\left(x, g_{T}(x) v\right)=\psi_{\beta}^{\prime-1}\left(x, g_{\beta^{\prime} \beta}(x) g_{T}(x) v\right)
$$

logo fazendo $v \rightarrow\left(g_{\beta^{\prime} \beta}\right)^{-1}(v)$ vemos que $g_{T}^{\prime}(x)=g_{\beta^{\prime} \beta}(x) g_{T}(x)\left(g_{\beta^{\prime} \beta}\right)^{-1} \in G \subset \mathbf{G L}(k)$.

O princípio da teoria de gauge é de que campos sejam seções de $G$-fibrados, e de que as leis da física sejam equações diferenciais, tais que se $s$ for uma seção solução dessas equações, então $g s$ também o deve ser, para toda transformação de gauge $g$.

POdemos, tomando outra atitude em relação a fibrados vetoriais focada em sua trivialidade local, construir $E$ colando os fibrados triviais $U_{\alpha} \times F$ através dos $g_{\alpha \beta}$ com as propriedades acima. A projeção $\pi$ é definida por

$$
\begin{gathered}
\pi: E \rightarrow V \\
{[x, v]_{\alpha} \mapsto x}
\end{gathered}
$$

As propriedades sobre $g_{\alpha \beta}$ garantem que, se $x \in U_{\alpha} \cap U_{\beta}$, então $(x, v) \in U_{\alpha} \times F$. Logo, se $(x, w) \in U_{\beta} \times F$, temos a seguinte relação de equivalência:

$$
(x, v) \sim(q, w) \Leftrightarrow x=g ; w=g_{\alpha \beta} v
$$

Isto é: 
(i) $(x, v) \sim(x, v) \Rightarrow g_{\alpha \alpha}=1$

(ii) $(x, v) \sim(x, w) \Rightarrow(x, w) \sim(x, v) \Rightarrow g_{\alpha \beta} g_{\beta \alpha}=1$ E finalmente

(iii) $(x, v) \sim(x, w) \sim(x, u) \Rightarrow(x, v) \sim(x, u) \Rightarrow g_{\alpha \gamma}=g_{\alpha \beta} g_{\beta \gamma} \Rightarrow[x, v]_{\alpha}=[q, w]_{\beta} \Leftrightarrow$ $x=q, v=g_{\alpha \beta} w$

E tomamos a estrutura vetorial em $\pi^{-1}(x)$ como: $\left[x, g_{\alpha \beta} U\right]_{\beta}+\left[x, g_{\alpha \beta} w\right]_{\beta}=[x, u]_{\alpha}+$ $[x, w]_{\alpha}=[x, u+w]_{\alpha}=\left[x, g_{\alpha \beta}(u+w)\right]_{\beta}$ já que $g_{\alpha \beta} \in \rho(G) \subset G L(F)$

Fazemos $E=\left(\bigcup_{\alpha \in \Lambda} U_{\alpha} \times F\right) / \sim$ e temos que, sobre cada $U_{\alpha}, E$ é trivial, isto é: temos o isomorfismo

$$
\begin{array}{r}
\Phi_{\alpha}: \pi^{-1}\left(U_{\alpha}\right) \rightarrow U_{\alpha} \times F \\
{[x, u]_{\alpha} \mapsto(x, u)}
\end{array}
$$

Dessa forma, temos se $x \in U_{\alpha} \cap U_{\beta}$, então $\Phi_{\alpha} \circ \Phi_{\beta}^{-1}(x, u)=\left(x, g_{\alpha \beta} u\right)$ e completamos a volta.

Se $S$ é alguma estrutura em $\mathbb{R}^{k}$ invariante por $G$, então podemos passar suavemente $S$ para cada $E_{x}$ pelos isomorfismos

$$
F_{x}^{i}: \mathbb{R}^{k} \rightarrow E_{x}
$$

Por exemplo uma estrutura riemanniana em $\mathbb{R}^{k}$ é invariante pelo grupo $O(k)$, portanto se $E$ for um $O(k)$-fibrado; podemos induzir suavemente uma estrutura riemanniana em $E$ por qualquer gauge (já que a transição de gauge está no grupo).

Um atlas de um $G$-fibrado é uma cobertura aberta $\left\{\theta_{\alpha}\right\}_{\alpha \in A}$ de $\mathrm{M}$ em conjunto com os mapas $g_{\alpha \beta}: \theta_{\alpha} \cap \theta_{p} \rightarrow G$ satisfazendo a condição de cociclos.

Dado um $G$-fibrado $E$, então um $G$-referencial para $E$ em $x$ é um isomorfismo linear dado por um gauge $F_{x}^{\theta}: \mathbb{R}^{k} \rightarrow E_{x}$. Dado um tal $G$-referencial $f_{0}$ nós temos que $f=f_{0} \circ g$ é também um $G$-referencial para todo $g \in G$ e o mapa $g \rightarrow f_{0} \circ g$ é uma bijeção de $G$ com o conjunto de todos os $G$-referenciais de $E$ em $x$.

$\operatorname{Aut}(E)$ é o grupo de automorfismos (isto é: isomorfismos de fibrados vetoriais entre $E$ e $E$ ) e se $E$ é um $G$-fibrado vetorial, então $\operatorname{Aut}_{G}(E)$ denota o sub-grupo de automorfismos de $E$ como $G$-fibrado vetorial, i.e.: tal que para $\psi \in \Gamma(\operatorname{Aut}(E)), \psi(x)=\rho(g)$, onde $g \in G$ e $\rho: G \rightarrow \operatorname{Aut}\left(E_{x}\right)$.

Se $E_{1}$ e $E_{2}$ forem fibrados sobre $M$, os elementos de $\Gamma\left(E_{1} \otimes E_{2}\right)$ são gerados pelos elementos da forma $s^{1} \otimes s^{2}$ onde $s^{1} \in \Gamma\left(E_{1}\right)$ e $s^{2} \in \Gamma\left(E_{2}\right)$. Vejamos porque: dados dois espaços vetoriais $V, W$, definimos $V \otimes W$ como a soma bilinear de elementos da forma ${ }^{3}$ $(v, w), v \in V ; w \in W$. É fácil ver que dadas bases $\left\{e_{k}\right\}_{k=1}^{\operatorname{dim}(V)}$ e $\left\{b_{i}\right\}_{i=1}^{\operatorname{dim}(W)}$ de $V$ e $W$, tanto $v$ quanto $w$ tem representações únicas em termos destas bases (fato elementar de álgebra linear), logo escrevendo $v$ e $w$ por extenso e utilizando a bilinearidade, cada elemento $(v, w)$ da soma se decompõe em uma combinação linear de elementos da base $\left(b_{i}, e_{k}\right)$. Juntando os

\footnotetext{
${ }^{3}$ I.e.: identificando em $V \times W$ os elementos, para $v_{1}, v_{2} \in V, w_{1}, w_{2} \in W$ :

- $\left(v, w_{1}+w_{2}\right)=\left(v, w_{1}\right)+\left(v, w_{2}\right)$

- $\left(v_{1}+v_{2}, w\right)=\left(v_{1}, w\right)+\left(v_{2}, w\right)$
} 
coeficientes de cada $i, k$ obtemos uma combinação linear de elementos $\left\{\left(b_{i}, e_{k}\right)\right\}$. Portanto voltando ao caso dos fibrados vetoriais $E_{1}$ e $E_{2}$, se $\left\{e_{i}\right\}_{i=1}^{k}$ e $\left\{b_{i}\right\}_{i=1}^{l}$ são bases locais de seções (referenciais) de, respectivamente, $E_{1}$ e $E_{2}$ sobre $\theta$, elas geram univocamente as seções locais sobre o fibrado produto. Obviamente nós temos que para $\psi \in \operatorname{Aut}_{G}\left(E_{1} \otimes E_{2}\right)$, então, para $x \in M, \psi(x)=\rho(g)$ onde $\rho$ é uma representação linear

$$
\rho: G \rightarrow L\left(\left(\left.\left.E_{1}\right|_{x} \otimes E_{2}\right|_{x}\right) ;\left(\left.\left.E_{1}\right|_{x} \otimes E_{2}\right|_{x}\right)\right)
$$

Como exemplo, vamos destrinchar o caso do fibrado $\operatorname{End}(E)$, que como mostraremos corresponde a $E_{1}=E, E_{2}=E^{*}$. Primeiramente, alguns resultados elementares de álgebra linear : Suponhamos $T: V \rightarrow W$ isomorfismo linear, como $T^{*}: W^{*} \rightarrow V^{*}$, o isomorfismo linear induzido por $T$ que leva $V^{*}$ em $W^{*}$ é justamente $\left(T^{-1}\right)^{*}$. Portanto, para $\lambda \in V^{*}$, $w \in W$, temos que $T$ age sobre $\lambda$ da forma natural (generalizado pelo pull-back):

$$
\left(T^{-1}\right)^{*} \lambda(w)=\lambda T^{-1}(w) \Rightarrow\left(T^{-1}\right)^{*} \lambda=\lambda T^{-1}
$$

Portanto se $\Omega=\lambda \otimes v \in V \otimes V^{*}$ a ação natural de um isomorfismo linear $T \in \operatorname{Aut}(V)$ é pela aplicação adjunta: $\Omega \rightarrow T \Omega T^{-1}$. Portanto se $\rho$ for uma representação de $G$ em $V$, a representação correspondente em $V \otimes V^{*}$ é pela representação adjunta

$$
\widetilde{\rho}(g): \Omega \rightarrow \rho(g) \Omega(\rho(g))^{-1}
$$

Nós temos ainda que existe um isomorfismo natural entre $L(V ; V)$ e $V \otimes V^{*}$. Afirmamos que existe um isomorfismo canônico $L(V ; V) \simeq V \otimes V^{*}$, dado pela matriz resultante de aplicação de $A \in L(V ; V)$ em uma base de $V$, i.e.: tomando base $e=\left\{e_{i}\right\}_{i=1}^{n}$ de $V$, sua dual $\left\{e^{i}\right\}_{i=1}^{n}=e^{*}$, base de $V^{*}$, escrevemos

$$
A=A_{j}^{i} e_{i} \otimes e^{j} \text { onde } A_{j}^{i}=e^{i}\left(A\left(e_{j}\right)\right)
$$

Para $T \in \operatorname{Aut}(V)$, tomando as bases induzidas naturalmente por $T, \tilde{e}=\left\{\tilde{e}_{i}\right\}_{i=1}^{n}=\left\{T e_{i}\right\}_{i=1}^{n}$ e $\tilde{e}^{*}=\left\{\tilde{e}^{i}\right\}_{i=1}^{n}=\left\{e^{i} T^{-1}\right\}_{i=1}^{n}$, em $V$ e $V^{*}$ respectivamente, obtemos que o isomorfismo independe de base simplesmente aplicando a definição (1.15) para as duas bases e utilizando (1.14) .

Portanto induzimos um isomorfismo natural independente de base $\operatorname{End}(V) \simeq V \otimes V^{*}$ (significando que a ação de uma transformação linear independe da base em que é representada), ou seja, $\operatorname{End}(E) \simeq E \otimes E^{*}$. O que significa que uma seção $s \in \Gamma(\operatorname{End}(E))$ sob um isomorfismo de fibrados $g: M \rightarrow \operatorname{Aut}(E)$ sofre a seguinte transformação: $s(x) \rightarrow g(x) s(x) g^{-1}(x) \in$ $\operatorname{End}\left(E_{x}\right)$. Ou seja, nesse caso as transformações de gauge agem pela ação adjunta dos automorfismos, na notação mais completa, se $\rho_{x}: G \rightarrow \operatorname{Aut}\left(E_{x}\right)$ é a representação usual do grupo sobre $E_{x}$, nós temos a nova representação correspondente: $\widetilde{\rho}_{x}(g(x))=\operatorname{Ad}(\rho(g(x)))$.

\subsection{Operadores Diferenciais Lineares}

Definimos $\alpha=\left(\alpha_{1}, \cdot, \alpha_{n}\right) \in\left(\mathbb{Z}^{+}\right)^{n}$ e $|\alpha|=\alpha_{1}+\cdots+\alpha_{n}$. Definimos ainda:

$$
D^{\alpha}:=\partial_{\alpha}=\frac{\partial^{|\alpha|}}{\partial x_{1}^{\alpha_{1}} \cdots \partial x_{n}^{\alpha_{n}}}: C^{\infty}\left(\mathbb{R}^{n} ; \mathbb{R}^{k}\right) \rightarrow C^{\infty}\left(\mathbb{R}^{n} ; \mathbb{R}^{k}\right)
$$


Uma aplicação linear $L: C^{\infty}\left(\mathbb{R}^{n} ; \mathbb{R}^{k}\right) \rightarrow C^{\infty}\left(\mathbb{R}^{n} ; \mathbb{R}^{l}\right)$ é chamada de operador diferencial de ordem menor ou igual a $r$ se é da forma:

$$
(L f)(x)=\sum_{|\alpha| \leq r} a_{\alpha}(x)\left(D^{\alpha} f\right)(x)
$$

onde $a_{\alpha} \in C^{\infty}\left(\mathbb{R}^{n} ; L\left(\mathbb{R}^{k}, \mathbb{R}^{l}\right)\right), f \in C^{\infty}\left(\mathbb{R}^{n} ; \mathbb{R}^{k}\right)$ e $D^{\alpha} f(x) \in \mathbb{R}^{k}$. Se $L$ tem ordem menor ou igual a $r$ e não tem ordem menor ou igual a $r-1, L$ é dito pertencer a $\operatorname{Diff}^{r}\left(\mathbb{R}^{n} \times \mathbb{R}^{k} ; \mathbb{R}^{n} \times \mathbb{R}^{l}\right)$.

Sejam então $\left(E, \pi_{E}, M\right)$ e $\left(F, \pi_{F}, M\right)$ fibrados vetoriais sobre M. Definimos que $L$ : $\Gamma(E) \rightarrow \Gamma(F)$ é um operador diferencial linear entre $E$ e $F$ de ordem no máximo igual a $r$ se é um morfismo entre $E$ e $F$ que pode localmente (i.e.: no do domínio de gauges) ser representado na forma de (1.16). Nós precisamos mostrar que essa definição independe das trivializações. Em primeiro lugar, precisamos escrever o que significa um operador diferencial ser representado localmente na forma de (1.16).

Sejam então $\theta$ aberto em $M$, tal que $\pi_{E}^{-1}(\theta)$ e $\pi_{F}^{-1}(\theta)$ são domínios de trivializações de gauge $\phi: \pi_{E}^{-1}(\theta) \rightarrow \theta \times \mathbb{R}^{k}$ e $\psi: \pi_{F}^{-1}(\theta) \rightarrow \theta \times \mathbb{R}^{l}$. Chamemos de $p r_{2}^{\phi}: \theta \times \mathbb{R}^{k} \rightarrow \mathbb{R}^{k}$ e $p r_{2}^{\psi}: \theta \times \mathbb{R}^{l} \rightarrow \mathbb{R}^{l}$ as projeções canônicas nas fibras típicas e $a_{\alpha} \in C^{\infty}\left(\theta ; L\left(\mathbb{R}^{k}, \mathbb{R}^{l}\right)\right)$.Então $L: \Gamma\left(E_{1}\right) \rightarrow \Gamma\left(E_{2}\right)$ é localmente representado na forma de (1.16) se, para toda seção $s \in \Gamma\left(E_{1}\right)$ e todo $m \in \theta$ :

$$
p r_{2}^{\psi} \circ \psi \circ L(s(m))=\sum_{|\alpha| \leq r} a_{\alpha}(m) D^{\alpha}\left(p r_{2}^{\phi} \circ \phi(s)\right)(m)
$$

Como para $m \in \theta$, nós temos $\psi_{m}:=p r_{2}^{\psi} \circ \psi_{\mid \pi_{F}^{-1}(m)}: \pi_{F}^{-1}(m) \rightarrow \mathbb{R}^{l}$ isomorfismo linear (e $\phi_{m}$ definido analogamente),

$$
L(s)(m)=\psi_{m}^{-1}\left(\sum_{|\alpha| \leq r} a_{\alpha}(m) D^{\alpha}\left(p r_{2}^{\phi} \circ \phi(s)\right)(m)\right)
$$

Sejam agora, definidos da mesma forma, trivializações locais $\widetilde{\psi}$ e $\widetilde{\phi}$. Temos então

$$
L(s)(m)=\widetilde{\psi}_{m}^{-1}\left(\sum_{|\alpha| \leq r}\left(\widetilde{\psi}_{m} \circ \psi_{m}^{-1}\right) \circ a_{\alpha}(m) D^{\alpha}\left(p r_{2}^{\phi} \circ \phi(s)\right)(m)\right)
$$

Mas por (1.4), $\left(\widetilde{\psi}_{m} \circ \psi_{m}^{-1}\right)=g_{\tilde{\psi} \psi}(m)$ Agora, chamando de $g_{\phi \tilde{\phi}}: \theta \rightarrow G L(k)$ o mapa de transição entre as trivializações $\phi$ e $\widetilde{\phi}$, nós temos, para qualquer $m \in \theta$ :

$$
\begin{aligned}
p r_{2}^{\phi} \circ \phi(s(m)) & =\left(p r_{2}^{\phi} \circ \phi\right)\left(\widetilde{\phi}^{-1} \circ \widetilde{\phi}\right)(s(m)) \\
& =p r_{2}^{\phi}\left(\phi \circ \widetilde{\phi}^{-1}\right)\left(m, p r_{2}^{\tilde{\phi}} \circ \widetilde{\phi}(s(m))\right) \\
& =p r_{2}^{\phi}\left(m, g_{\phi \tilde{\phi}} p r_{2}^{\tilde{\phi}} \circ \widetilde{\phi}(s(m))\right) \\
& =g_{\phi \tilde{\phi}}(m) p r_{2}^{\tilde{\phi}} \circ \widetilde{\phi}(s(m))
\end{aligned}
$$


podemos reescrever (1.19):

$$
L(s)(m)=\tilde{\psi}_{m}^{-1}\left(\sum_{|\alpha| \leq r} g_{\tilde{\psi} \psi}(m) \circ a_{\alpha}(m) D^{\alpha}\left(g_{\phi \tilde{\phi}}\left(p r_{2}^{\widetilde{\phi}} \circ \widetilde{\phi}(s)\right)\right)(m)\right)
$$

Utilizando regra da cadeia :

$$
L(s)(m)=\tilde{\psi}_{m}^{-1}\left(\sum_{|\alpha| \leq r} g_{\tilde{\psi} \psi}(m) \circ a_{\alpha}(m) \sum_{|\gamma| \leq|\alpha|}\left(\begin{array}{l}
|\alpha| \\
|\gamma|
\end{array}\right) D^{\alpha-\gamma}\left(g_{\phi \tilde{\phi}}\right)(m) D^{\gamma}\left(p r_{2}^{\tilde{\phi}} \circ \widetilde{\phi}(s)\right)(m)\right)
$$

onde a notação simplificada subsume que $\gamma$ é tal que $\gamma^{i} \leq \alpha^{i}$. Mas $g_{\phi \tilde{\phi}} \in C^{\infty}\left(\theta ; L\left(\mathbb{R}^{k}, \mathbb{R}^{k}\right)\right) \simeq$ $C^{\infty}\left(\theta ; \mathbb{R}^{k^{2}}\right)$, portanto $D^{\alpha-\gamma}\left(g_{\phi \tilde{\phi}}\right) \in C^{\infty}\left(\theta ; L\left(\mathbb{R}^{k}, \mathbb{R}^{k}\right)\right)$. Finalmente

$$
L(s)(m)=\tilde{\psi}_{m}^{-1}\left(\sum_{|\gamma| \leq r}\left(\sum_{r \geq|\alpha| \geq|\gamma|} g_{\tilde{\psi} \psi}(m) \circ a_{\alpha}(m)\left(\begin{array}{c}
|\alpha| \\
|\gamma|
\end{array}\right) D^{\alpha-\gamma}\left(g_{\phi \tilde{\phi}}\right)(m)\right) D^{\gamma}\left(p r_{2}^{\tilde{\phi}} \circ \widetilde{\phi}(s)\right)(m)\right)
$$

como $g_{\tilde{\psi} \psi} \in C^{\infty}\left(\theta ; L\left(\mathbb{R}^{l}, \mathbb{R}^{l}\right)\right)$ nós temos que

$$
\left(\sum_{r \geq|\alpha| \geq|\gamma|} g_{\tilde{\psi} \psi}(m) \circ a_{\alpha}(m)\left(\begin{array}{c}
|\alpha| \\
|\gamma|
\end{array}\right) D^{\alpha-\gamma}\left(g_{\phi \tilde{\phi}}\right)(m)\right)=: \tilde{a}_{\gamma} \in C^{\infty}\left(\theta ; L\left(\mathbb{R}^{k}, \mathbb{R}^{l}\right)\right)
$$

$\operatorname{logo} \widetilde{a}_{\gamma} \in C^{\infty}\left(\theta ; L\left(\mathbb{R}^{k}, \mathbb{R}^{l}\right)\right)$ e portanto verificamos que vale

$$
L(s)(m)=\widetilde{\psi}_{m}^{-1}\left(\sum_{|\gamma| \leq r} \widetilde{a}_{\gamma}(m) D^{\gamma}\left(p r_{2}^{\tilde{\phi}} \circ \widetilde{\phi}(s)\right)(m)\right)
$$

\section{Adjuntos Formais de Operadores Diferenciais}

Sejam $E, F$ fibrados vetoriais riemannianos sobre $M$. O subespaço de $\Gamma(E)$ composto por seções de suporte compacto será denotado por $\Gamma_{C}(E)$. Se $s_{1}, s_{2} \in \Gamma_{C}(E)$, então é claro que $x \rightarrow\left\langle s_{1}(x), s_{2}(x)\right\rangle$ tem suporte compacto. Logo, utilizando o produto interno pontual em $E$ $\langle\cdot, \cdot\rangle$, definimos o produto interno em $\Gamma_{C}(E)$, denotado $\langle\langle\cdot, \cdot\rangle\rangle$, por

$$
\left\langle\left\langle s_{1}, s_{2}\right\rangle\right\rangle=\int_{M}\left\langle s_{1}, s_{2}\right\rangle \mu
$$

É trivial mostrar que é bilinear e positivo definido, já que $\langle\cdot, \cdot\rangle$ o é, e pela suavidade das seções elas não podem ser não nulas em um conjunto de medida zero. Agora, se $L: \Gamma(E) \rightarrow \Gamma(F)$ é tal que $L \in \operatorname{Diff}^{r}(E, F)$, então chamamos de adjunto formal de $L$, o operador diferencial linear $L^{*}: \Gamma(F) \rightarrow \Gamma(E)$, tal que valha, para todos $s_{1} \in \Gamma_{C}(E)$ e $s_{2} \in \Gamma_{C}(F)$ :

$$
\left\langle\left\langle L\left(s_{1}\right), s_{2}\right\rangle\right\rangle=\int_{M}\left\langle L\left(s_{1}\right), s_{2}\right\rangle_{F} \mu=\int_{M}\left\langle s_{1}, L^{*}\left(s_{2}\right)\right\rangle_{E} \mu=\left\langle\left\langle s_{1}, L^{*}\left(s_{2}\right)\right\rangle\right\rangle
$$


É claro que se tal operador adjunto existir, pela não degenerescência do produto interno acima, ele será único.

Como vimos, para toda seção $s_{1} \in \Gamma(E)$ e para $m \in \theta$, por $(1.18) L: \Gamma(E) \rightarrow \Gamma(F)$ pode ser escrito como :

$$
L\left(s_{1}\right)(m)=\psi_{m}^{-1}\left(\sum_{|\alpha| \leq r} a_{\alpha}(m) D^{\alpha}\left(p r_{2}^{\phi} \circ \phi\left(s_{1}\right)\right)(m)\right)
$$

Como $\psi_{m}$ é isometria ,para $u, v \in \mathbb{R}^{l}$ :

$$
\langle u, v\rangle_{\mathbf{R}^{l}}=\left\langle\psi_{m}^{-1}(u), \psi_{m}^{-1}(v)\right\rangle_{F}
$$

Agora, como é isometria, o adjunto de $\psi_{m}^{-1}$ é igual a $\psi_{m}$ e nós obtemos:

$$
\begin{aligned}
\left\langle L\left(s_{1}\right)(m), s_{2}(m)\right\rangle_{F} & \left.=\left\langle\psi_{m}^{-1} \sum_{|\alpha| \leq r} a_{\alpha}(m) D^{\alpha}\left(p r_{2}^{\phi} \circ \phi\left(s_{1}\right)\right)(m), s_{2}(m)\right)\right\rangle_{F} \\
& =\left\langle\sum_{|\alpha| \leq r} a_{\alpha}(m) D^{\alpha}\left(p r_{2}^{\phi} \circ \phi\left(s_{1}\right)\right)(m),\left(\psi_{m} \circ s_{2}(m)\right\rangle_{\mathbb{R}^{l}}\right.
\end{aligned}
$$

Como $a_{\alpha}(m) \in L\left(\mathbb{R}^{k} ; \mathbb{R}^{l}\right)$, podemos tomar também o seu adjunto $a_{\alpha}(m)^{*}$, utilizando então a bilinearidade da métrica obtemos:

$$
\left\langle L\left(s_{1}\right)(m), s_{2}(m)\right\rangle_{F}=\sum_{|\alpha| \leq r}\left\langle D^{\alpha}\left(p r_{2}^{\phi} \circ \phi\left(s_{1}\right)\right)(m), a_{\alpha}(m)^{*} \circ \psi_{m}\left(s_{2}(m)\right)\right\rangle_{\mathbf{R}^{k}}
$$

Integrando sobre $\theta$ e utilizando integração por partes sucessivamente obtemos:

$$
\int_{\theta}\left\langle L\left(s_{1}\right), s_{2}\right\rangle_{F} \mu=\sum_{|\alpha| \leq r} \int_{\theta}(-1)^{|\alpha|}\left\langle\phi_{m}\left(s_{1}(m)\right), D^{\alpha}\left(a_{\alpha}^{*}\left(p r_{2}^{\psi} \circ \psi\right) \circ s_{2}\right)(m)\right\rangle_{\mathbb{R}^{k}} \mu
$$

E finalmente:

$$
\int_{\theta}\left\langle L\left(s_{1}\right), s_{2}\right\rangle_{F} \mu=\int_{\theta}\left\langle s_{1}(m), \sum_{|\alpha| \leq r}(-1)^{|\alpha|} \phi_{m}^{-1} D^{\alpha}\left(a_{\alpha}^{*}\left(p r_{2}^{\psi} \circ \psi\right) \circ s_{2}\right)(m)\right\rangle_{\mathbf{R}^{k}} \mu
$$

Onde o operador ao lado direito é claramente um morfismo entre $F$ e $E$. Portanto provamos que localmente existe um adjunto formal. Se tivermos dois adjuntos formais sobre $\theta, L_{\mid \theta}^{*}, \widetilde{L}_{\mid \theta}^{*}$ então claramente

$$
\frac{1}{2}\left(L_{\mid \theta}^{*}+\widetilde{L}_{\mid \theta}^{*}\right)
$$

é também um adjunto formal, ou seja, combinações lineares convexas de adjuntos formais locais são adjuntos formais locais. Portanto, como $L(s)_{\mid U}=L\left(s_{\mid U}\right)$, tomando uma partição da unidade subordinada a uma cobertura de $M$ por abertos que sejam domínios de trivializações locais obtemos um adjunto formal global, que como mencionamos é único. Calcularemos explicitamente alguns adjuntos formais ao longo da exposição. 


\section{Formas Diferenciais a Valores em Um Fibrado Vetorial}

Se $V$ é um espaço vetorial denotamos por $\Lambda^{p}(V)$, todas as aplicações anti-simétricas p-lineares de $V$ em $\mathbb{R}$. Se $W$ é um espaço vetorial, então $\Lambda^{p}(V) \otimes W$ denota o espaço das formas a valores em $W$, e é gerado linearmente por elementos da forma $\eta \otimes w$ onde $\eta \in \Lambda^{p}(V)$ e $w \in W$. Sejam então $v_{1}, \cdots, v_{p} \in V$, então nós temos que

$$
\eta \otimes w\left(v_{1}, \cdots, v_{p}\right):=\eta\left(v_{1}, \cdots, v_{p}\right) w
$$

que é alternante p-linear.

Se $E$ é um fibrado sobre $M$ chamamos $\Lambda^{p}\left(T M^{*}\right) \otimes E$ de fibrado de p-formas em $M$ a valores em $E$. Notemos que se $\lambda \in \Gamma\left(\Lambda^{p}\left(T M^{*}\right) \otimes E\right)$, então para todo $x \in M, \lambda_{x} \in \Lambda^{p}\left(T_{x} M\right) \otimes$ $E_{x}$ é um mapa alternante p-linear de $T_{x} M$ em $E_{x}$. Agora, $\Gamma\left(\Lambda^{p}\left(T M^{*}\right) \otimes E\right)$ também é gerado $C^{\infty}(M, \mathbb{R})$-linearmente por elementos da forma $\eta \otimes s$, onde $\eta \in \Gamma\left(\Lambda^{p}\left(T M^{*}\right)\right)$ e $s \in \Gamma(E)$, logo se $X_{1}, \cdots, X_{p} \in \Gamma(T M)$, nós temos que

$$
\eta \otimes s\left(X_{1}, \cdots, X_{p}\right)=\eta\left(X_{1}, \cdots, X_{p}\right) s
$$

e como

$$
\eta\left(X_{1}, \cdots, X_{p}\right) \in C^{\infty}(M) \Rightarrow \eta \otimes s\left(X_{1}, \cdots, X_{p}\right) \in \Gamma(E)
$$

Isto é, se $\lambda \in \Gamma\left(\Lambda^{p}\left(T M^{*}\right) \otimes E\right)$, a aplicação:

$$
\begin{array}{r}
\lambda\left(X_{1}, \cdots, X_{p}\right): M \rightarrow E \\
x \mapsto \lambda_{x}\left(\left(X_{1}\right)_{x}, \cdots,\left(X_{p}\right)_{x}\right)
\end{array}
$$

é uma seção de $E$, p-linear e anti-simétrica nos $X_{1}, \cdots, X_{p}$ (já que a é em cada ponto).

Agora sejam $\eta^{1} \in \Gamma\left(\Lambda^{p_{1}}\left(T M^{*}\right) \otimes E_{1}\right)$ e $\lambda^{2} \in \Gamma\left(\Lambda^{p_{2}}\left(T M^{*}\right) \otimes E_{2}\right)$, definimos

$$
\lambda^{1} \tilde{\wedge} \lambda^{2} \in \Gamma\left(\Lambda^{p_{1}+p_{2}}\left(T M^{*}\right) \otimes E_{1} \otimes E_{2}\right)
$$

por

$\lambda^{1} \tilde{\wedge} \lambda^{2}\left(X_{1}, \cdots, X_{p_{1}+p_{2}}\right)=\frac{p_{1} ! p_{2} !}{\left(p_{1}+p_{2}\right) !} \sum_{\sigma \in P\left(p_{1}+p_{2}\right)} \tau(\sigma) \lambda^{1}\left(X_{\sigma(1)}, \cdots, X_{\sigma\left(p_{1}\right)}\right) \otimes \lambda^{2}\left(X_{\sigma\left(p_{1}+1\right)}, \cdots, X_{\sigma\left(p_{1}+p_{2}\right)}\right)$

Onde $P\left(p_{1}+p_{2}\right)$ é o grupo de permutações de $p_{1}+p_{2}$ elementos, e $\tau(\sigma)$ é a paridade da permutação $\sigma$. Em outras palavras, o operador $\tilde{\Lambda}$ age como produto externo só na parte de formas da seção. Se chamarmos o produto externo usual de

$$
\wedge_{\mathbb{R}}=\Gamma\left(\Lambda^{p_{1}}\left(T M^{*}\right)\right) \times \Gamma\left(\Lambda^{p_{2}}\left(T M^{*}\right)\right) \rightarrow \Gamma\left(\Lambda^{p_{1}+p_{2}}\left(T M^{*}\right)\right)
$$

temos, para elementos da forma $\eta^{1} \otimes s^{1}$ onde $\eta^{1} \in \Gamma\left(\Lambda^{p_{1}}\left(T M^{*}\right)\right)$ e $s^{1} \in \Gamma\left(E_{1}\right)$ que a equação (1.25) fica:

$$
\left(\eta^{1} \otimes s^{1}\right) \tilde{\Lambda}\left(\eta^{2} \otimes s^{2}\right)=\left(\eta^{1} \wedge_{\mathbb{R}} \eta^{2}\right) \otimes\left(s^{1} \otimes s^{2}\right)
$$


Mais rigorosamente, seja $\left\{\eta^{i}\right\}_{i=1}^{n}$ base local de $\Gamma\left(\Lambda^{1}\left(T M^{*}\right)\right)$ e $\left\{e_{j}\right\}_{i=1}^{k}$ base local de $\Gamma\left(\left.E_{1}\right|_{\theta}\right)$. Lembramos que $\left.E_{1}\right|_{\theta}:=\pi_{1}^{-1}(\theta)$ onde $\pi_{1}: E_{1} \rightarrow M$. Nós temos que $\left\{\eta^{I}\right\}$ é base local de $\Gamma\left(\Lambda^{p_{1}}\left(\left.T M^{*}\right|_{\theta}\right)\right)$, onde o superscrito maiúsculo $I$ é a chamada notação de multi-índices, que denota uma combinação de $p_{1}$ elementos da forma $\eta^{i}$. I.e.: se $I=\left(i_{1}, \cdots, i_{p_{1}}\right)$ com $1 \leq i_{1}<\cdots<i_{p_{1}} \leq n$ então $\eta^{I}=\eta^{i_{1}} \wedge \cdots \wedge \eta^{i_{p_{1}}}$.

Logo $\Gamma\left(\Lambda^{p_{1}}\left(T \bar{M}^{*}\right) \otimes E_{1}\right)$ é localmente gerado $C^{\infty}(M, \mathbb{R})$-linearmente pela base $\left\{\eta^{I} \otimes e_{j}\right\}$ de forma única. Seja $J=\left(j_{1}, \cdots, j_{p_{2}}\right)$, e $\left\{b_{j}\right\}_{i=1}^{l}$ base local de $\Gamma\left(\left.E_{2}\right|_{\theta}\right)$, temos então, para $\lambda^{1} \in \Gamma\left(\left.\Lambda^{p_{1}}\left(\left.T M^{*}\right|_{\theta}\right) \otimes E_{1}\right|_{\theta}\right)$ e $\lambda^{2} \in \Gamma\left(\left.\Lambda^{p_{2}}\left(\left.T M^{*}\right|_{\theta}\right) \otimes E_{2}\right|_{\theta}\right), \lambda^{1}=f_{I}^{i} \eta^{I} \otimes e_{i}, \lambda^{2}=g_{J}^{j} \eta^{J} \otimes b_{j}$, $f_{I}^{i}, g_{J}^{j}: \theta \rightarrow \mathbb{R}$. Portanto, obtemos :

$$
\lambda^{1} \tilde{\wedge} \lambda^{2}=f_{I}^{i} g_{J}^{j}\left(\eta^{I} \wedge_{\mathbf{R}} \eta^{J}\right) \otimes\left(e_{i} \otimes b_{j}\right)
$$

No caso de $p_{1}=p_{2}=1$ então

$$
\lambda^{1} \tilde{\wedge} \lambda^{2}\left(X_{1}, X_{2}\right)=\frac{1}{2}\left(\lambda^{1}\left(X_{1}\right) \otimes \lambda^{2}\left(X_{2}\right)-\lambda^{1}\left(X_{2}\right) \otimes \lambda^{2}\left(X_{1}\right)\right) \in \Gamma\left(E_{1}\right) \otimes \Gamma\left(E_{2}\right)
$$

Onde $X_{1}, X_{2}$ são seções de $T M$. Se tivermos no entanto uma aplicação bilinear $\mu$ : $E_{1} \otimes E_{2} \rightarrow E_{3}$, ao invés de $\tilde{\Lambda}$, substituindo o produto tensorial em (1.28) por $\mu$, podemos definir um produto externo $\Lambda$, que vai de formas a valores em $E_{1}$ e $E_{2}$, respectivamente, em $\Gamma\left(\Lambda^{p_{1}+p_{2}}\left(T M^{*}\right) \otimes E_{3}\right)$. I.e.:

$$
\wedge: \Gamma\left(\Lambda^{p_{1}}\left(T M^{*}\right) \otimes E_{1}\right) \otimes \Gamma\left(\Lambda^{p_{2}}\left(T M^{*}\right) \otimes E_{2}\right) \rightarrow \Gamma\left(\Lambda^{p_{1}+p_{2}}\left(T M^{*}\right) \otimes E_{3}\right)
$$

E para o caso de 1-formas:

$$
\lambda^{1} \wedge \lambda^{2}\left(X_{1}, X_{2}\right)=\frac{1}{2}\left(\mu\left(\lambda^{1}\left(X_{1}\right), \lambda^{2}\left(X_{2}\right)\right)-\mu\left(\lambda^{1}\left(X_{2}\right), \lambda^{2}\left(X_{1}\right)\right)\right)
$$

Por exemplo, podemos ter $E_{1}=E$ e $E_{2}=E^{*} \operatorname{com} \mu=C$ sendo o operador de contração e nesse caso $E_{3}=M \times \mathbb{R}$. Ou ainda, $E_{1}=E_{2}=E$ e $\mu=g$ uma métrica sobre $E$ (já que cada fibra é linear e portanto comporta produto interno), novamente com $E_{3}=M \times \mathbb{R}$. Outro exemplo bastante útil é se $E_{1}=M \times \mathbb{R}$, ou seja, $p$-formas a valores reais, neste caso $\mu$ é simplesmente a multiplicação por funções reais.

Mas o caso mais importante para nós é se $E_{1}=E_{2}=\operatorname{End}(E)$, o grupo dos endomorfismos de $E$. Nesse caso temos uma aplicação natural de composição de endomorfismos $\operatorname{End}(E) \times$ $\operatorname{End}(E) \rightarrow \operatorname{End}(E)$.

Em termos de uma base $\left\{s_{k}^{1}\right\}=\left\{s_{k}^{2}\right\}=\left\{e^{i} \otimes e_{j}\right\}$ onde $\left\{e_{i}\right\}$ é base local de $\Gamma\left(\left.E\right|_{\theta}\right)$ e $\left\{e^{i}\right\}$ é sua base dual, base de $\Gamma\left(\left.E^{*}\right|_{\theta}\right)$, para $\lambda^{1} \in \Gamma\left(\left.\Lambda^{p}\left(\left.T M^{*}\right|_{\theta}\right) \otimes \operatorname{End}(E)\right|_{\theta}\right), \lambda^{1}=\lambda^{1^{i}}{ }_{j} \otimes e_{i} \otimes e^{j}$ onde $\lambda^{1^{i}} \in \Gamma\left(\Lambda^{p}\left(\left.T M^{*}\right|_{\theta}\right)\right)$ podemos ilustrar a operação acima como uma simples contração:

$$
\lambda^{1} \wedge \lambda^{2}=\lambda^{1^{i}}{ }_{j} \wedge_{\mathbb{R}} \lambda^{2^{l}}{ }_{k} e_{i} \otimes\left(e^{j}\left(e_{l}\right)\right) \otimes e^{k}=\lambda^{1^{i}}{ }_{j} \wedge_{\mathbb{R}} \lambda^{2 j} e_{i} \otimes e^{k}
$$

Fazemos aqui a importante observação que, salvo aviso, tomaremos sempre este produto exterior entre formas a valores em $\operatorname{End}(E)$. 
Agora, quando $E_{1}=E_{2}=E$, se o fibrado for um fibrado de álgebras, existe uma aplicação $E \otimes E \rightarrow E$. Com essa aplicação podemos novamente definir um produto externo a valores em $E$ e (1.28) pode tomar a forma:

$$
\lambda^{1} \tilde{\wedge} \lambda^{2}\left(X_{1}, X_{2}\right)=\frac{1}{2}\left(\lambda^{1}\left(X_{1}\right) \lambda^{2}\left(X_{2}\right)-\lambda^{1}\left(X_{2}\right) \lambda^{2}\left(X_{1}\right)\right)
$$

Pela forma de (1.31), a notação para tal aplicação no fibrado se sugere como:

$$
\lambda^{1} \tilde{\wedge} \lambda^{2}\left(X_{1}, X_{2}\right)=\left[\lambda^{1}\left(X_{1}\right), \lambda^{2}\left(X_{2}\right)\right]=\left[\lambda^{1}, \lambda^{2}\right]\left(X_{1}, X_{2}\right)
$$

Em particular, se for um fibrado de àlgebras anti-comutativas:

$$
\lambda\left(X_{1}\right) \lambda\left(X_{2}\right)=-\lambda\left(X_{2}\right) \lambda\left(X_{1}\right) \Longrightarrow \lambda \wedge \lambda=\lambda \otimes \lambda
$$

Portanto fica aqui claro que não temos, como no caso da formas a valores reais (que é um fibrado de àlgebras comutativas), que $\lambda \wedge \lambda=0$. Em geral, temos localmente, em termos de uma base $\left\{e_{i}\right\}$ de $\mathfrak{g}, \lambda=\lambda^{i} e_{i}$, denotando o produto da álgebra por $[\cdot, \cdot]$ :

$$
\begin{aligned}
\lambda \wedge \lambda\left(X_{1}, X_{2}\right) & =\frac{1}{2}\left(\lambda\left(X_{1}\right) \lambda\left(X_{2}\right)-\lambda\left(X_{2}\right) \lambda\left(X_{1}\right)\right) \\
& =\lambda^{i}\left(X_{1}\right) \lambda^{j}\left(X_{2}\right) e_{i} e_{j}-\lambda^{j}\left(X_{2}\right) \lambda^{i}\left(X_{1}\right) e_{j} e_{i} \\
& =\lambda^{i}\left(X_{1}\right) \lambda^{j}\left(X_{2}\right)\left[e_{i}, e_{j}\right]
\end{aligned}
$$

\section{A Derivada Exterior}

Seja $E=M \times V$, neste caso (já que podemos manter seções de $\Gamma(E)$ "constantes"), teremos um operador diferencial de primeira ordem:

$$
\begin{gathered}
d: \Gamma\left(\Lambda^{p}(M) \otimes E\right) \rightarrow \Gamma\left(\Lambda^{p+1} \otimes E\right) \\
\lambda \longmapsto d \lambda
\end{gathered}
$$

Onde para, $X_{1}, \cdots, X_{p+1} \in \Gamma(T M)$,

$$
\begin{aligned}
d \lambda\left(X_{1}, \cdots, X_{p+1}\right)= & \sum_{i=1}^{p+1}(-1)^{i+1} X_{i} \lambda\left(X_{1}, \cdots, \hat{X}_{i}, \cdots, X_{p+1}\right)+ \\
& \sum_{1 \leq i \leq j \leq p+1}(-1)^{i+j} \lambda\left(\left[X_{i}, X_{j}\right], X_{1}, \cdots, \hat{X}_{i}, \cdots, \hat{X}_{j}, \cdots, X_{p+1}\right)
\end{aligned}
$$

Que é exatamente análogo à definição da derivada exterior de $p$-formas a valores reais, e generaliza

$$
d \lambda(X, Y)=X[\lambda(Y)]-Y[\lambda(X)]-\lambda([X, Y])
$$


Mostremos que $d \lambda$ é $C^{\infty}(M)$-multilinear, como a definição é obviamente anti-simétrica, basta que provemos em uma entrada. Seja então $f \in C^{\infty}(M)$ :

$$
\begin{aligned}
d \lambda\left(f X_{1}, \cdots, X_{p+1}\right) & =f X_{1}\left[\lambda\left(X_{2}, \cdots, X_{p+1}\right)\right] \\
& +\sum_{i=2}^{p+1}(-1)^{i+1} X_{i}\left[\lambda\left(f X_{1}, \cdots, \hat{X}_{i}, \cdots, X_{p+1}\right)\right] \\
& +\sum_{i=2}^{p+1}(-1)^{i+1} \lambda\left(\left[f X_{1}, X_{i}\right], \cdots, \hat{X}_{i}, \cdots, X_{p+1}\right) \\
& +\sum_{2 \leq i \leq j \leq p+1}(-1)^{i+j} \lambda\left(\left[X_{i}, X_{j}\right], f X_{1}, \cdots, \hat{X}_{i}, \cdots, \hat{X}_{j}, \cdots, X_{p+1}\right)
\end{aligned}
$$

Mas $\lambda$ é multilinear, então

$$
\begin{aligned}
\sum_{i=2}^{p+1}(-1)^{i+1} X_{i}\left[\lambda\left(f X_{1}, \cdots, \hat{X}_{i}, \cdots, X_{p+1}\right)\right] & =\sum_{i=2}^{p+1}(-1)^{i+1} X_{i}[f] \lambda\left(X_{1}, \cdots, \hat{X}_{i}, \cdots, X_{p+1}\right) \\
& +f \sum_{i=2}^{p+1}(-1)^{i+1} X_{i}\left[\lambda\left(X_{1}, \cdots, \hat{X}_{i}, \cdots, X_{p+1}\right)\right]
\end{aligned}
$$

Além disso, como $\left[f X_{1}, X_{i}\right]=f\left[X_{1}, X_{i}\right]-X_{i}[f] X_{1}$,

$$
\begin{gathered}
\sum_{i=2}^{p+1}(-1)^{i+1} \lambda\left(\left[f X_{1}, X_{i}\right], \cdots, \hat{X}_{i}, \cdots, X_{p+1}\right) \\
=f \sum_{i=2}^{p+1}(-1)^{i+1} \lambda\left(\left[X_{1}, X_{i}\right], \cdots, \hat{X}_{i}, \cdots, X_{p+1}\right)-\sum_{i=2}^{p+1}(-1)^{i+1} X_{i}[f] \lambda\left(X_{1}, \cdots, \hat{X}_{i}, \cdots, X_{p+1}\right)
\end{gathered}
$$

Juntando todos os termos obtemos

$$
d \lambda\left(f X_{1}, \cdots, X_{p+1}\right)=f d \lambda\left(X_{1}, \cdots, X_{p+1}\right)
$$

Logo $d \lambda$ é tensorial, só depende dos valores dos campos nos pontos calculados.Temos as seguintes propriedades, idênticas àquelas válidas para formas a valores reais:

(i) $d$ é linear

(ii) $d\left(\lambda^{1} \wedge \lambda^{2}\right)=d \lambda^{1} \wedge \lambda^{2}+(-1)^{p_{1}} \lambda^{1} \wedge d \lambda^{2}$,para $\left.\lambda^{i} \in \Gamma\left(\Lambda^{p_{i}}\left(T M^{*}\right) \otimes E\right)\right)$.

(iii) $d^{2}=0$

(iv) Se $\lambda$ tem suporte compacto $C$ então

$$
\int_{C} d \lambda=\int_{\partial C} \lambda
$$

(Teorema de Stokes). 
Bem coloquialmente, o que é a derivada exterior? É a potencialidade de variação. A potencialidade de variação de uma função por exemplo, se toma ao longo de direções, e a de uma função sobre direções (1-formas) se toma ao longo de elementos de área direcionados ${ }^{4}$ Agora, porque a potencialidade de variação $d a$ potencialidade de variação é nula? Porque a potencialidade de variação não é uma quantia escalar, e sua direção em pontos (ou elementos de área, etc.) vizinhos é oposta [4]. Pense em uma distribuição de temperatura, se você vai de $A$ para $B$ a temperatura aumenta tanto quanto diminui se você toma a direção oposta, de $B$ pra $A$. Portanto a soma das potencialidades se cancela, deixando somente o valor na fronteira da região tomada (Teorema de Stokes). Como a fronteira de uma fronteira é nula, a integral

$$
\int_{S} d^{2} \eta=0
$$

para qualquer $p$-forma $\eta$ e qualquer variedade $S$, logo $d^{2}=0$.

\subsection{Teorema de Decomposição de Hodge}

\section{O Operador $*$ de Hodge}

Suponhamos que $M^{n}$ tenha estrutura Riemanniana $(M,\langle\cdot, \cdot\rangle)$, há uma maneira natural de induzir um isomorfismo entre $T_{x} M$ e $T_{x} M^{*}$ dado pela métrica, a saber, dado $\lambda \in T_{x} M^{*}$ e $u, v \in T_{x} M$ definimos $u^{\sharp} \in T_{x} M^{*}$ por $\langle u, \cdot\rangle$ :

$$
u^{\sharp}(v)=\langle u, v\rangle
$$

assumindo que a métrica é não degenerada, e que o espaço dual tem a mesma dimensão, é fácil verificar que $\sharp: T_{x} M \rightarrow T_{x} M^{*}$ é isomorfismo: se $u, v \in T_{x} M$ tal que $\langle u, w\rangle=\langle v, w\rangle$ para todo $w \in T_{x} M$, então

$$
\langle u-v, w\rangle=0 \Longrightarrow u-v=0
$$

Denotamos o inverso de $\sharp$ por $b: T_{x} M^{*} \rightarrow T_{x} M$. Podemos vizualizar este isomorfismo da seguinte maneira: dado um vetor $v \in T_{x} M, v^{\sharp}$ seria representado por uma "pilha" de hiperplanos em $T_{x} M$ ortogonais a $v$, de forma que $v^{\sharp}(w)$ fosse a velocidade com que o vetor $w$ atravessa os hiperplanos. Dessa maneira, se $w$ é ortogonal a $v, v^{\sharp}(w)=0$. Claramente precisamos da métrica para nos dizer o que é ortogonalidade.

O produto exterior de duas 1-formas, seguindo esse raciocínio, seria uma família de vetores (elementos de linha), dadas pela intersecções dos seus respectivos hiperplanos, e sua aplicação feita em elementos de área (correspondentes a dois vetores), seria a velocidade com a qual esses elementos de linha atravessam esses elementos de área [5]. Em dimensões mais altas a vizualização se torna totalmente abstrata, mas, seguindo esse raciocínio, uma $p$-forma em um ponto $x \in M$ seria equivalente a uma pilha de subespaços $n-p$ dimensionais em $T_{x} M$, e sua aplicação a $p$ vetores ordenados seria a velocidade com que a pilha é "atravessada" pelos subespaços orientados $p$ dimensionais formados por esses vetores.

\footnotetext{
${ }^{4}$ As características Grassmanianas de 2 -formas vêm de serem relacionadas não a um elemento de área qualquer, e sim a um elemento de área direcionado.
} 
O produto interno em $T_{x} M$ induz um produto interno em $\Lambda^{p}\left(T_{x} M\right)$. Como o produto interno deve ser uma aplicação bilinear, basta definirmos tal aplicação em elementos formados pelo produto externo de $p$ 1-formas. Seja então $\lambda_{i}, \theta_{i} \in \Lambda^{1}\left(T_{x} M\right)$, denotaremos o produto interno em $\Lambda^{p}\left(T_{x} M\right)$ por $\leqslant \cdot, \ldots, \cdot \geqslant$ :

$$
\leqslant \lambda_{1} \wedge \cdots \wedge \lambda_{p}, \theta_{1} \wedge \cdots \wedge \theta_{p} \geqslant:=\sum_{\sigma \in S_{n}} \tau(\sigma)\left\langle\lambda_{\sigma(1)}, \theta_{1}\right\rangle \cdots\left\langle\lambda_{\sigma(p)}, \theta_{p}\right\rangle=\operatorname{det}\left(\left\langle\lambda_{i}, \theta_{j}\right\rangle\right)
$$

onde definimos $\langle\lambda, \theta\rangle:=\left\langle\lambda^{b}, \theta^{b}\right\rangle, S_{p}$ denota o grupo de permutação de $p$ elementos, $\tau(\sigma)$ é a paridade da permutação $\sigma$. Se $\left\{e^{i}\right\}_{i=1}^{n}$ é uma base ortonormal para $T_{x}^{*} M$, então os $\left(\begin{array}{l}n \\ p\end{array}\right)$ elementos formados por $e^{I}$, onde o superscrito maiúsculo $I$ é um multi-índice de $p$ elementos ${ }^{5}$, forma uma base ortonormal para $\Lambda^{p}\left(T_{x} M\right)$.

Logo $\Lambda^{n}\left(T_{x} M\right)$ é 1-dimensional e tem dois elementos de norma 1. Se pudermos escolher $\mu \in \Gamma\left(\Lambda^{n}\left(T M^{*}\right)\right)$ tal que $\left\|\mu_{x}\right\|=1$, então $M$ é orientável e uma escolha é chamada de orientação de $M, \mu$ é o elemento de volume Riemanniano.

Consideremos o mapa bilinear:

$$
\begin{aligned}
& B_{p}: \Gamma\left(\Lambda^{p}\left(T M^{*}\right)\right) \times \Gamma\left(\Lambda^{n-p}\left(T M^{*}\right)\right) \rightarrow C^{\infty}(M, \mathbb{R}) \\
&(\lambda, \nu) \longmapsto B_{p}(\lambda, \nu) \mu=\lambda \wedge \nu
\end{aligned}
$$

Proposition $1 B_{p}$ é não-degenerado e portanto determina unicamente um isomorfismo *: $\Gamma\left(\Lambda^{p}\left(T M^{*}\right)\right) \rightarrow \Gamma\left(\Lambda^{n-p}\left(T M^{*}\right)\right)$ tal que:

$$
\lambda \wedge * \nu=\leqslant \lambda, \nu \geqslant \mu
$$

onde $\lambda, \nu \in \Lambda^{p}(M), \mu \in \Lambda^{n}(M)$.

Dem: Seja $\left\{e_{i}\right\}_{i=1}^{n}$ base ortonormal de $T_{x} M$, e $I=\left(i_{1}, \cdots, i_{p}\right)$ com $1 \leq i_{1}<\cdots<$ $i_{p} \leq n$, chamamos de $I^{C}$ o complementar de $I$ em $(1,2, \cdots, n)$ em ordem também crescente. Chamaremos novamente de $\tau(I)$ a paridade da permutação levando $(1,2, \cdots, n)$ em $\left(i_{1}, i_{2}, \cdots, i_{n}\right)$ :

$$
\left(\begin{array}{ccccccc}
1 & 2 & \cdots & p & p+1 & \cdots & n \\
i_{1} & i_{2} & \cdots & i_{p} & j_{1} & \cdots & j_{n-p}
\end{array}\right)
$$

obviamente $e_{I} \wedge e_{I^{c}}=\tau(I) \mu$. Nós temos ainda que para qualquer $J$ subconjunto crescente de $n-p$ elementos de $\{1, \cdots, n\}$ tal que $J \neq I^{c}$, necessariamente $e_{I} \wedge e_{J}=0$. Logo se $I \neq J$ então $e_{I} c \neq e_{J C}$ e portanto $\leqslant e_{I^{C}}, e_{J^{C}} \geqslant=0$.

Chamando de $\mathcal{C}$ a coleção de todos os subconjuntos crescentes de p-elementos de $\{1, \cdots, n\}$, nós temos então que $\left\{e_{I}\right\}_{I \in C}$ e $\left\{\tau(I) e_{I^{c}}\right\}_{I \in C}$ são bases ortonormais de $\Lambda^{p}\left(T_{x} M\right)$ e $\Lambda^{n-p}\left(T_{x} M\right)$ (já que têm a mesma dimensão). Agora, como $*$ deve satisfazer:

$$
e_{I} \wedge * e_{I}=\leqslant e_{I}, e_{I} \geqslant \mu=\mu=\tau(I) \tau(I) \mu=e_{I} \wedge \tau(I) e_{I^{C}}
$$

podemos definir o operador linear * como agindo em uma base da seguinte forma

$$
* e_{I}:=\tau(I) e_{I} c
$$

\footnotetext{
${ }^{5}$ I.e.: se $I=\left(i_{1}, \cdots, i_{p}\right) \operatorname{com} 1 \leq i_{1}<\cdots<i_{p} \leq n$ então $\lambda^{I}=\lambda^{i_{1}} \wedge \cdots \wedge \lambda^{i_{p}}$.
} 
Como leva base em base, o operador * é isomorfismo linear, que por construção obedece (1.39), como $B_{p}$ é não degenerado, o operador estábem definido e é único. Claramente se $e_{I}=\mu$, então $* e_{I}=1$.

Aqui nossa maneira de visualizar formas como elementos de área direcionados vem a calhar. Todo subespaço de um espaço vetorial tem um subespaço ortogonal, mas somente subespaços direcionados (com orientação) têm subespaços ortogonais direcionados, é daí que vem todas as caracaterísticas do operador de Hodge. Similarmente ao operador $\sharp$ podemos encarar o operador $*$ de Hodge como levando cada elemento de área $p$-dimensional orientado ao elemento de área $(n-p)$-dimensional ortogonal de orientação compatível. Por exemplo, em $\mathbb{R}^{3}$ com a métrica canônica:

$$
* d x=d y \wedge d z, \quad * d y=d z \wedge d x, \quad * d z=d x \wedge d y
$$

Continuando, $* e_{I}=\tau(I) e_{I^{c}}$ então

$$
*\left(* e_{I}\right)=\tau(I) \tau\left(I^{c}\right) e_{I}=\tau\left(I \cdot I^{c}\right) e_{I}
$$

que é a permutação:

$$
\left(\begin{array}{ccccccc}
i_{1} & i_{2} & \cdots & i_{p} & j_{1} & \cdots & j_{n-p} \\
j_{1} & \cdots & j_{n-p} & i_{1} & \cdots & i_{p}
\end{array}\right)
$$

cuja paridade é $(-1)^{p(n-p)}$. Em particular se $p=\frac{n}{2}$ e $n=4 m \Rightarrow p(n-p)=4 m^{2}$ então $(-1)^{p(n-p)}=1$.

Portanto, $(*)^{2}=I d$, e como $*: \Lambda^{p}\left(T_{x} M\right): \rightarrow \Lambda^{p}\left(T_{x} M\right)$ logo temos, para $\lambda \in \Lambda^{p}\left(T_{x} M\right)$,

$$
\begin{aligned}
\lambda & =\frac{1}{2}\left(\lambda_{+}+\lambda_{-}\right) \\
& =\frac{1}{2}((\lambda+* \lambda)+(\lambda-* \lambda)) \\
\therefore \quad * \lambda & =\frac{1}{2}\left(* \lambda_{+}+* \lambda_{-}\right) \\
& =\frac{1}{2}((* \lambda+\lambda)+(* \lambda-\lambda)) \\
& =\frac{1}{2}\left(\lambda_{+}-\lambda_{-}\right)
\end{aligned}
$$

Então denotando o autoespaço do autovalor $k$ do operador linear $*$ no espaço em questão como $A(k)$, nós obtemos $\Lambda^{p}\left(T_{x} M\right)=A(-1) \oplus A(1)$, decomposição que é importante no estudo das equações de Yang-Mills.

Agora, para $E$ fibrado vetorial Riemanniano, utilizando o produto externo nestes espaços que incorpora o produto interno riemanniano nas fibras (que denotaremos nesse caso por $g$ ), como explicitado na equação (1.29), se $\lambda \in \Lambda^{p}\left(T_{x} M\right) \otimes E_{x}$ e $\nu \in \Lambda^{n-p}\left(T_{x} M\right) \otimes E_{x}$ pela equação (1.25) definimos este produto externo como:

$$
\lambda \wedge \nu\left(X_{1}, \cdots, X_{n}\right):=\frac{p !(n-p) !}{n !} \sum_{\sigma \in P(n)} \tau(\sigma) g\left(\lambda\left(X_{\sigma(1)}, \cdots, X_{\sigma(p)}\right), \nu\left(X_{\sigma(p+1)}, \cdots, X_{\sigma(n)}\right)\right)
$$


Então assim como para formas a valores reais, incorporando o produto interno riemanniano obtemos uma forma bilinear não degenerada de $\left(\Lambda^{p}\left(T_{x} M\right) \otimes E_{x}\right) \otimes\left(\Lambda^{n-p}\left(T_{x} M\right) \otimes E_{x}\right) \rightarrow$ $\mathbb{R}$ :

$$
\lambda \wedge \nu=B_{p}(\lambda, \nu) \mu \in \Lambda^{n}\left(T_{x} M\right)
$$

Seguindo a demonstração do lema anterior, obtemos um único isomorfismo $*: \Lambda^{p}(M) \otimes E \rightarrow$ $\Lambda^{n-p}(M) \otimes E$ caracterizado por

$$
\lambda \wedge * \nu=\langle\lambda, \nu\rangle \mu
$$

a saber, o isomorfismo levando base ortonormal de $\Lambda^{p}\left(T_{x} M\right) \otimes E_{x}$ em base ortonormal de $\Lambda^{n-p}\left(T_{x} M\right) \otimes E_{x}:$

$$
*\left(e_{I} \otimes b_{j}\right)=\tau(I) e_{I^{c}} \otimes b_{j}
$$

onde $\lambda \in \Lambda^{p}(M) \otimes E$ e o produto interno, para o qual utilizamos a mesma notação do produto interno de 1-formas, aqui incorpora tanto o produto interno pontual para $p$-formas, $\leqslant \cdot, \cdot \geqslant$, quanto o produto interno pontual riemanniano.

\section{Coderivada Exterior}

Pela definição, se $\lambda, \nu \in \Gamma_{C}\left(\Lambda^{p}(M) \otimes E\right)$

$$
\langle\langle\lambda, \nu\rangle\rangle=\int_{M}\langle\lambda, \nu\rangle \mu=\int_{M} \lambda \wedge * \nu
$$

Vamos calcular explicitamente o adjunto formal da derivada exterior $d: \Gamma\left(\Lambda^{p}\left(T M^{*}\right) \otimes\right.$ $E) \rightarrow \Gamma\left(\Lambda^{p+1}\left(T M^{*}\right) \otimes E\right)$, que chamaremos de $\delta: \Gamma\left(\Lambda^{p+1}\left(T M^{*}\right) \otimes E\right) \rightarrow \Gamma\left(\Lambda^{p}\left(T M^{*}\right) \otimes E\right)$, a coderivada exterior. Lembramos antes de mais nada que só existe um conceito natural de derivada exterior sobre fibrados produto $E=M \times V$ (ou localmente para a trivialização $\theta \times V)$, já que aí há uma maneira natural de manter um campo "fixo".

Seja $\lambda \in \Gamma_{C}\left(\Lambda^{p}\left(T M^{*}\right) \otimes E\right)$ e $\nu \in \Gamma_{C}\left(\Lambda^{p+1}\left(T M^{*}\right) \otimes E\right)$. Lembramos que $* \nu \in \Gamma_{C}\left(\Lambda^{n-p-1}\left(T M^{*}\right) \otimes\right.$ $E)$ e portanto $\lambda \wedge * \nu \in \Gamma_{C}\left(\Lambda^{n-1}\left(T M^{*}\right)\right)$ e $d(\lambda \wedge * \nu) \in \Gamma_{C}\left(\Lambda^{n}\left(T M^{*}\right)\right)$. Portanto podemos utilizar Stokes:

$$
\begin{aligned}
\int_{M} d(\lambda \wedge * \nu) & =\int_{M} d \lambda \wedge * \nu+\int_{M}(-1)^{p} \lambda \wedge d(* \nu) \\
& =\int_{\partial M} \lambda \wedge * \nu=0 \\
\therefore \quad \int_{M} d \lambda \wedge * \nu & =\int_{M}(-1)^{(p+1)} \lambda \wedge d(* \nu)
\end{aligned}
$$

Mas pela definição de $*$, sobre cada ponto de $M, d \lambda \wedge * \nu=\langle d \lambda, \nu\rangle \mu$, e por outro lado, como $* *=(-1)^{p(n-p)}$ então $(-1)^{p(n-p)} * *=1$, e nós temos

$$
\begin{gathered}
(-1)^{p+1} \lambda \wedge d(* \nu)=(-1)^{p+1} \lambda \wedge\left((-1)^{p(n-p)} * * d(* \nu)\right)=(-1)^{p+p(n-p)+1} \lambda \wedge *(* d(* \nu)) \\
\therefore \quad(-1)^{p+1} \lambda \wedge d(* \nu)=(-1)^{p+p(n-p)+1}\langle\lambda, * d(* \nu)\rangle \mu
\end{gathered}
$$

Subsituindo em (1.48), obtemos

$$
\int_{M}\langle d \lambda, \nu\rangle \mu=(-1)^{p+p(n-p)+1} \int_{M}\langle\lambda, * d(* \nu)\rangle \mu
$$




\section{Então}

$$
\langle\langle d \lambda, \nu\rangle\rangle=(-1)^{p+p(n-p)+1}\langle\langle\lambda, * d * \nu\rangle\rangle
$$

e portanto

$$
\delta_{p+1}=(-1)^{n p+1} *_{n-p} d_{n-(p+1)} *_{p+1}
$$

onde utilizamos que $-1^{p^{2}-p}=1$ e os subscritos denotam o grau das formas a que os operadores estão sendo aplicados; a coderivada exterior está sendo aplicada em $p+1$-formas a valores no fibrado e as levando para $p$ formas a valores no fibrado. Então finalmente obtemos para $\delta: \Gamma\left(\Lambda^{p}\left(T M^{*}\right) \otimes E\right) \rightarrow \Gamma\left(\Lambda^{p-1}\left(T M^{*}\right) \otimes E\right)$

$$
\delta_{p}=(-1)^{n(p+1)+1} *_{n-p+1} d_{n-p} *_{p}
$$

Temos as seguintes propriedades para a coderivada exterior, facilmente verificáveis (aplicaremos em formas de grau $p$ ):

(i) $\delta \circ \delta=d * \delta=\delta * d=0$

Como $d$ e $*$ são lineares, $\delta \circ \delta= \pm * d \circ d *= \pm d * \delta= \pm \delta * d=0$.

(ii) $* d \delta=\delta d *, * \delta d=d \delta *$

Simplesmente escrevendo por extenso os dois lados das equações obtemos os resultados.

(iii) $\delta_{n-p} *_{p}=(-1)^{p+1} * d$

Nós temos $\delta_{n-p} *_{p}=(-1)^{n(n-p+1)+1+p(n-p)} *_{p+1} d_{p}$, e fazendo as contas obtemos $(-1)^{n(n-p+1)+1+p(n-p)}=(-1)^{p+1}$

(iv) $*_{p-1} \delta_{p}=(-1)^{p} d *$

Já que $(-1)^{(p-1)(n-p+1)+n(p+1)+1}=(-1)^{p}$

\section{O Laplaciano}

Em matemática e física, o Laplaciano, denotado por $\Delta$, é um operador diferencial de suma importância, sendo utilizado na modelagem de propagação de ondas e fluxo de calor. É ainda central na teoria eletromagnética e na mecânica quântica, onde representa o operador de energia cinética. Definido como o divergente do gradiente, em coordenadas cartesianas de $\mathbb{R}^{3}$ o operador assume a bem conhecida fórmula:

$$
\widetilde{\Delta}=\frac{\partial^{2}}{\partial x^{2}}+\frac{\partial^{2}}{\partial y^{2}}+\frac{\partial^{2}}{\partial z^{2}}=\sum_{i=1}^{3} \partial_{i}^{2}
$$

É possível provar que em uma variedade Riemmaniana $M$ qualquer, podemos escrever o operador de Laplace acima, a menos de um sinal negativo, como ${ }^{6}$

\footnotetext{
${ }^{6} \mathrm{Na}$ notação mais comum entre os físicos, o negativo do divergente do gradiente em uma variedade riemanniana $M$ aplicado em $f \in C^{\infty}(M, \mathbb{R})$ é escrito como:
}

$$
\Delta(f)=-\tilde{\Delta}(f)=-\frac{1}{\sqrt{|g|}} \partial_{i}\left(\sqrt{|g|} \partial^{i} f\right)
$$




$$
\Delta_{p}=d_{p-1} \delta_{p}+\delta_{p+1} d_{p}
$$

ou em notação mais compacta: $\Delta=d \delta+\delta d$, chamaremos este operador de Laplaciano, ao invés de $\widetilde{\Delta}$, preferência justificada por ser assim um operador positivo definido, como mostramos a seguir. É trivial perceber que adjunto do Laplaciano, $\Delta^{*}=\delta d+d \delta=\Delta$, ou seja, é um operador auto-adjunto. O núcleo de $\Delta$ é chamado de espaço de $p$-formas harmônicas a valores em $V$.

Proposition 2 Temos as seguintes propriedades do Laplaciano:

(i) O laplaciano é auto-adjunto.

(ii) Para $\lambda \in \Gamma_{C}\left(\Lambda^{p}\left(T^{*} M\right) \otimes E\right)$, $\lambda$ é harmônico se e somente se tivermos ambas as condições: $d \lambda=0$ e $\delta \lambda=0$.

(iii) $* \Delta=\Delta *$. Logo se $\lambda$ é harmônica então $* \lambda$ também é.

Dem: Já comentamos o primeiro item. Para o segundo, supondo que $\lambda$ é harmônica temos:

$$
\begin{array}{rlc}
d(\delta \lambda)+\delta(d \lambda)=0 & \Longrightarrow & \langle\langle d(\delta \lambda)+\delta(d \lambda), \lambda\rangle\rangle=0 \\
& =\langle\langle d(\delta \lambda), \lambda\rangle\rangle+\langle\langle\delta(d \lambda), \lambda\rangle\rangle \\
& = & \langle\langle\delta \lambda, \delta \lambda\rangle\rangle+\langle\langle d \lambda, d \lambda\rangle\rangle \\
& \therefore & \delta \lambda=0 \text { e } d \lambda=0
\end{array}
$$

onde utilizamos na última passagem que o produto interno $\langle\langle\cdot, \cdot\rangle\rangle$ é definido positivo. A afirmação de (ii) é óbvia. Fica claro também dessa demonstração que o Laplaciano é assim positivo definido.

Para o terceiro item, basta observar o item (ii) das propriedades da coderivada exterior acima.

De agora em diante, chamaremos $\Gamma_{C}\left(\Lambda^{p}\left(T^{*} M\right) \otimes E\right)$ de $\mathcal{A}^{p}(E)$ ou abreviando ainda mais, $\mathcal{A}^{p}$, e o espaço de $p$-formas harmônicas em $\mathcal{A}^{p}$ de $\mathcal{H}^{p}$. Um corolário trivial desta última proposição é o Teorema de Liouville, que diz que se $M$ é compacto, orientado e conexo, então qualquer função harmônica, i.e.: tal que $\Delta f=0$, é constante (já que $d f=0$ ). Temos ainda que se $\lambda$ for uma $n$-forma harmônica, então $\lambda$ é um múltiplo constante da forma volume, já que $\lambda=f \mu$ e portanto $* \Delta \lambda=\Delta * \lambda=\Delta f=d f=0$.

Proposition $3 \mathcal{H}^{p}, \operatorname{Im}\left(d_{p-1}\right)$ e $\operatorname{Im}\left(\delta_{p+1}\right)$ são mutuamente ortogonais em $\mathcal{A}^{p}$.

utilizindo a notação física: $|g|=\operatorname{det}\left(g_{i j}\right)$ e $g^{i j} g_{j k}=\delta_{k}^{i}$ é a matriz inversa da métrica, e $\partial^{i}=g^{i j} \partial_{j}$ é o levantamento do campo $\partial_{i}$ pela métrica, i.e.: $\partial_{i}^{\sharp}$. Utilizando novamente a notação de multi-índices para letras maiúsculas, $\mu$ como a forma volume temos:

$$
\begin{gathered}
\Delta f=d \delta f+\delta d f=\delta d f=\delta \partial_{i} f d x^{i}=-* d * \partial_{i} f d x^{i}=-* d\left(\tau(i J) \sqrt{|g|} \partial^{i} f d x^{J}\right) \\
=-* \tau(i J) \partial_{j}\left(\sqrt{|g|} \partial^{i} f\right) d x^{j} d x^{J}=-* \frac{1}{\sqrt{|g|}} \partial_{i}\left(\sqrt{|g|} \partial^{i} f\right) \mu=-\frac{1}{\sqrt{|g|}} \partial_{i}\left(\sqrt{|g|} \partial^{i} f\right)
\end{gathered}
$$


Dem: Seja $\lambda \in \mathcal{A}^{p-1}, \nu \in \mathcal{A}^{p+1}$ e $\eta \in \mathrm{H}^{p}$.

- $\operatorname{Im}\left(d_{p-1}\right) \perp \operatorname{Im}\left(\delta_{p+1}\right)$ :

$$
\langle\langle d \lambda, \delta \nu\rangle\rangle=\langle\langle\lambda, \delta(\delta \nu)\rangle\rangle=0
$$

- $\operatorname{Im}\left(d_{p-1}\right) \perp \mathrm{H}^{p}$ :

$$
\langle\langle d \lambda, \eta\rangle\rangle=\langle\langle\lambda, \delta \eta\rangle\rangle=0
$$

- $\operatorname{Im}\left(\delta_{p+1}\right) \perp \mathrm{H}^{p}$ :

$$
\langle\langle\eta, \delta \nu\rangle\rangle=\langle\langle d \eta, \nu\rangle\rangle=0
$$

\section{O Teorema de Decomposição de Hodge}

Theorem 3 Para $M$ compacto, e $E=M \times V$ fibrado riemanniano sobre $M$. Então $\mathcal{A}^{p}=$ $\mathcal{H}^{p} \oplus \operatorname{Im}\left(d_{p-1}\right) \oplus \operatorname{Im}\left(\delta_{p+1}\right)$

Nós temos que $\mathcal{H}^{p} \oplus \operatorname{Im}\left(d_{p-1}\right) \oplus \operatorname{Im}\left(\delta_{p+1}\right)$ é uma soma não só direta, mas perpendicular, contida em $\mathcal{A}^{p}$. Infelizmente $\mathcal{A}^{p}$ é de dimensão infinita, e uma prova desse teorema involve uma incursão em análise funcional e cohomologia de Rham que não farermos aqui (ver [6], [9]). Se $\mathcal{A}^{p}$ fosse de dimensão finita seria suficiente provar que um elemento de $\mathcal{A}^{p}$ ortogonal a $\mathcal{H}^{p} \oplus \operatorname{Im}\left(d_{p-1}\right) \oplus \operatorname{Im}\left(\delta_{p+1}\right)$ é obrigatoriamente nulo. Isto é: se $\lambda \in \mathcal{A}^{p}$ é ortogonal a $\operatorname{Im}\left(d_{p-1}\right)$, nós temos para todo $\nu \in \mathcal{A}^{p-1}$,

$$
\langle\langle d \nu, \lambda\rangle\rangle=\langle\langle\nu, \delta \lambda\rangle\rangle=0
$$

portanto, como tomamos $\nu$ qualquer, $\delta \lambda=0$. Da mesma forma obtemos que se $\lambda$ é ortogonal a $\operatorname{Im}\left(\delta_{p+1}\right)$ então $d \lambda=0$. Agora, claramente se $\lambda$ for ortogonal a $\operatorname{Im}\left(d_{p-1}\right) \oplus \operatorname{Im}\left(\delta_{p+1}\right)$ então $\lambda \in \operatorname{Ker}\left(d_{p-1}\right) \oplus \operatorname{Ker}\left(\delta_{p+1}\right)=\mathcal{H}^{p}$, e claramente se for ortogonal aos três, $\lambda=0$. Agora assumiremos que para todo $\lambda$ ortogonal a $\mathcal{H}^{p}$ existe $\nu \in \mathcal{A}^{p}$ que satisfaz a equação:

$$
\Delta \nu=\lambda
$$

um fato advindo da teoria de equações diferenciais parciais elípticas [6].

Como o núcleo de qualquer operador linear é um subespaço fechado, e intersecções arbitrárias de conjuntos fechados é fechada, temos que $\mathcal{H}^{p}$ é fechado em um espaço normado, $\mathcal{A}^{p}$. Logo dado um elemento $\nu \in \mathcal{A}^{p}$, temos que existem muitos $h \in \mathcal{H}^{p}$ e $h^{\perp} \in \mathcal{A}^{p}-\mathcal{H}^{p}$ tais que $h^{\perp}=\nu-h$, mas únicos tais que a norma de $h^{\perp}=\nu-h$ é mínima, ou seja temos uma decomposição ortogonal $\mathcal{A}^{p}=\left(\mathcal{H}^{p}\right)^{\perp} \oplus \mathcal{H}^{p}$. Chamaremos a projeção em $\mathcal{H}$ de $\widehat{\mathcal{H}}$.

Para todo $\eta \in \mathcal{A}^{p}$ nós teremos que $\eta-\widehat{\mathcal{H}}(\eta) \in\left(\mathcal{H}^{p}\right)^{\perp}$ e portanto por hipótese existe $\nu \in \mathcal{A}^{p}$ tal que

$$
\Delta \nu=d(\delta \nu)+\delta(d \nu)=\eta-\widehat{\mathcal{H}}(\eta)
$$

e finalmente $\eta=d(\delta \nu)+\delta(d \nu)+\widehat{\mathcal{H}}(\eta)$ o que nos fornece a decomposição de Hodge.

Proposition 4 Se $\lambda \in \mathcal{A}^{p}$ é fechada, i.e.: $d \lambda=0$, então existe um único $h \in \mathcal{H}^{p}$ e um único $\nu \in \mathcal{A}^{p-1}$ tal que $\lambda=h+d \nu$. 
Dem: Pela decomposição de Hodge nós temos

$$
\lambda=h+d \nu+\delta \eta
$$

Mas como $d \lambda=0$, obtemos $d \delta \eta=0$. Logo

$$
\langle\langle d \delta \eta, \eta\rangle\rangle=\langle\langle\delta \eta, \delta \eta\rangle\rangle=0 \Longrightarrow \delta \eta=0
$$

e obtemos $\lambda=h+d \nu$, temos que cada classe de cohomologia contém um único representante harmônico.

$\mathrm{Na}$ verdade a recíproca também vale; o resultado do Teo.3 fornece uma resposta à seguinte pergunta: dada uma $p$-forma $\lambda \in \mathcal{A}^{p}$ em um fibrado trivial Riemanniano sobre uma variedade compacta $M$, sob quais condições existe $\nu \in \mathcal{A}^{p}$ que satisfaz a equação:

$$
\Delta \nu=\lambda ?
$$

A resposta é que a condição necessária e suficiente é $\lambda$ ser ortogonal a $\mathcal{H}^{p}$. Que é necessária é facilmente demonstrável: suponha que $\Delta \nu=\lambda$, então para todo $\eta \in \mathcal{H}^{p}$ temos:

$$
\langle\langle\eta, \lambda\rangle\rangle=\langle\langle\eta, \Delta \nu\rangle\rangle=\langle\langle\Delta \eta, \nu\rangle\rangle=0
$$

A suficiência deriva do Teorema de Decomposição de Hodge: Assumindo que $\lambda \in\left(\mathcal{H}^{p}\right)^{\perp}$ nós temos que $\lambda=d \nu+\delta \eta$. Agora afirmamos que existem $\theta, \alpha \in \mathcal{A}^{p}$ tal que $\Delta \theta=d \nu$ e $\Delta \alpha=\delta \eta$. De fato, sucessivamente aplicando o teorema de Hodge obtemos:

$$
\begin{gathered}
\nu=d \nu_{1}+\delta \eta_{1}+\gamma_{1} \Longrightarrow d \nu=d \delta \eta_{1} \\
\eta_{1}=d \nu_{2}+\delta \eta_{2}+\gamma_{2} \Longrightarrow \delta \eta_{1}=\delta d \nu_{2}
\end{gathered}
$$

portanto substituindo uma na outra: $d \nu=d \delta\left(d \nu_{2}\right)=\Delta\left(d \nu_{2}\right)=\Delta \theta$, onde o $\theta$ que procurávamos é dado por $d \nu_{2}$. E portanto, fazendo a mesma conta para $\eta$ obtemos, para algum $\alpha, \delta \eta=\Delta \alpha$ e finalmente $\lambda=\Delta(\alpha+\theta)$.

\subsection{Conexões em Fibrados Vetoriais}

Nós vimos que no caso de fibrados localmente triviais, ao redor de qualquer ponto da base há uma vizinhança sobre cujas fibras existe o conceito de uma seção se manter" constante". Poderíamos escolher comparar vetores segundo essa trivialização, nós teríamos um " pano de fundo" local em cada fibra segundo o qual poderíamos dizer se um campo variou ou permaneceu constante. Ainda assim, esse " pano de fundo" depende da trivialização. Uma seção de um fibrado designa para cada ponto da base um elemento da fibra sobre aquele ponto, e não existe forma canônica de comparação entre elementos de fibras diferentes, há muitas formas distintas de se fazer isto. Para comparar elementos de diferentes fibras nós precisamos de um isomorfismo entre estas fibras, precisamos escolher um pano de fundo, uma forma de comparação. No caso de uma trivialização, isto equivale a se utilizar da "estrutura produto" local e o isomorfismo natural das coordenadas do espaço produto para estabelecerse uma equivalência entre as fibras. A escolha de uma forma de comparação de valores entre diferentes espaços internos chama-se conexão. 
Definition 4 Uma conexão em um fibrado vetorial $E$ sobre $M$ é um mapa linear

$$
\nabla: \Gamma(E) \rightarrow \Gamma\left(T^{*} M \otimes E\right)
$$

tal que se $f \in C^{\infty}(M, \mathbb{R})$ e se $s \in \Gamma(E)$ então

$$
\nabla(f s)=f \nabla s+d f \otimes s
$$

Theorem 4 Qualquer que seja E fibrado vetorial sobre $M$, existe uma conexão em E.

Dem: Dividiremos nossa demonstração em quatro partes:

(i) Se $\phi: E_{1} \rightarrow E_{2}$ é um isomorfismo de fibrados vetoriais, i.e.: $\pi_{1} \circ \phi=\pi_{2}$ onde $\pi_{1}: E_{1} \rightarrow$ $M, \pi_{2}: E_{2} \rightarrow M$ e

$$
\phi_{\mid \pi_{1}^{-1}(x)}: \pi_{1}^{-1}(x) \rightarrow \pi_{2}^{-1}(x)
$$

é isomorfismo linear. Então seja

$$
{ }_{1} \nabla: \Gamma\left(E_{1}\right) \rightarrow \Gamma\left(T^{*} M \otimes E_{1}\right)
$$

uma conexão em $E_{1}$, e seja

$$
\tilde{\phi}: T^{*} M \otimes E_{1} \rightarrow T^{*} M \otimes E_{1}
$$

dada por $\tilde{\phi}=I d \otimes \phi$ (claramente $C^{\infty}$ bilinear).

Definimos então

$$
\begin{array}{r}
{ }_{2} \nabla: \Gamma\left(E_{2}\right) \rightarrow \Gamma\left(T^{*} M \otimes E_{2}\right) \\
s \mapsto \tilde{\phi} \circ\left({ }_{1} \nabla\left(\phi^{-1}(s)\right)\right)
\end{array}
$$

Agora seja $f \in C^{\infty}(M, \mathbb{R})$ e $s \in \Gamma\left(E_{2}\right)$ então temos

$$
\begin{gathered}
{ }_{2} \nabla(f s)=\tilde{\phi} \circ_{1} \nabla\left(\phi^{-1}(f s)\right)=\tilde{\phi}\left({ }_{1} \nabla f \phi^{-1}(s)\right) \\
=\tilde{\phi}\left(\left(d f \otimes \phi^{-1}(s)\right)+f\left({ }_{1} \nabla\left(\phi^{-1}(s)\right)\right)=d f \otimes s+f \tilde{\phi} \circ_{1} \nabla\left(\phi^{-1}(s)\right)\right. \\
=d f \otimes s+f\left({ }_{2} \nabla s\right)
\end{gathered}
$$

$\operatorname{Logo}_{2} \nabla$ é uma conexão em $\Gamma\left(E_{2}\right)$ induzida por $\phi$.

(ii) Agora se $E=M \times V \Rightarrow \Gamma(E)=C^{\infty}(M, V)$ e se $s \in \Gamma(E) \Rightarrow s=s^{i} e_{i}$ onde $s^{i} \in$ $C^{\infty}(M, \mathbb{R})$ e nós temos que se $f \in C^{\infty}(M, \mathbb{R}) \Rightarrow d\left(s^{i} e_{i}\right)=d s^{i} \otimes e_{i}$ é a conexão flat de $E: d\left(f s^{i} e_{i}\right)=d f \otimes s^{i} e_{i}+f d s^{i} \otimes e_{i}$

(iii) Pelos itens (i) e (ii) nós temos que um gauge $F: \theta \times \mathbb{R}^{k} \rightarrow E_{\mid \theta}$ define uma conexão $\nabla^{F}$ para $E_{\mid \theta}$. 
(iv) Agora seja $\left\{\theta_{\alpha}\right\}$ uma cobertura de $M$ localmente finita (e pequena suficiente para que cada $\theta_{\alpha}$ seja um domínio de uma carta de gauge) e $\left\{\phi_{\alpha}\right\}$ uma partição de unidade a ela subordinada. Chamamos de $\nabla^{\alpha}$ a conexão flat induzida pelo gauge em $E \mid \theta_{\alpha}$.

Seja $\nabla: \Gamma(E) \rightarrow \Gamma\left(T^{*} M \otimes E\right)$ dada por $\nabla=\sum_{\alpha} \phi_{\alpha} \nabla^{\alpha}$, então, para $f \in C^{\infty}(M, \mathbb{R})$ e $s \in \Gamma(E)$ nós temos

$$
\begin{aligned}
\nabla(f s)= & \sum_{\alpha} \phi_{\alpha} \nabla^{\alpha}(f s)=\sum_{\alpha} \phi_{\alpha}\left(d f \otimes s+f \nabla^{\alpha} s\right) \\
& =d f \otimes s+f \sum_{\alpha} \phi_{\alpha} \nabla^{\alpha} s=d f \otimes s+f \nabla s
\end{aligned}
$$

Agora definimos

$$
\begin{aligned}
\nabla_{X}: \Gamma(E) & \longrightarrow \Gamma(E) \\
s & \longmapsto \nabla s(X)
\end{aligned}
$$

Como $\nabla$ é linear, $\nabla_{X}$ também o é. Podemos ver isso facilmente localmente, já que

$$
\nabla s=a_{i}^{j} e^{i} \otimes s_{j} \Rightarrow \nabla s(f X)=f \nabla s(X)
$$

Resumindo nós temos as seguintes propriedades

- $\nabla_{X} \in \operatorname{Diff}^{1}(E, E)$ e é linear

- $X \longrightarrow \nabla_{X}$ é linear

- Por (1.51) e a definição de $\nabla_{X}$, temos $\nabla_{X} f s=X[f] s+f \nabla_{X} s$

\section{Curvatura de Uma Conexão}

Seja $E=\theta \times V, \nabla$ a conexão trivial vinda deste gauge, i.e.: $\nabla^{\phi}=d$. Se nós não conhecermos a trivialização específica de antemão, existe alguma forma de detectarmos que existe um gauge para o qual $\nabla$ tem a forma acima? Seja $f \in \Gamma(E) \simeq C^{\infty}(M, V)$ e $X, Y \in \Gamma(T M)$

$$
\begin{gathered}
\Rightarrow \nabla_{X} f=X[f] \Rightarrow\left[\nabla_{X}, \nabla_{Y}\right] f=[X, Y] f=\nabla_{[X, Y]} f \\
\therefore\left[\nabla_{X}, \nabla_{Y}\right]=\nabla_{[X, Y]}
\end{gathered}
$$

I.e.: $X \longrightarrow \nabla_{X}$ é um homomorfismo de álgebras de Lie entre $\Gamma(T M)$ em $\operatorname{Diff}(E, E)$, que é a condição pela qual definiremos uma conexão flat, ou plana. Em geral, este não será o caso, o que sugere que estudemos o mapa:

$$
\begin{array}{r}
\Omega: \Gamma(T M) \times \Gamma(T M) \rightarrow \operatorname{Diff}^{0}(E, E) \\
(X, Y) \longmapsto\left[\nabla_{X}, \nabla_{Y}\right]-\nabla_{[X, Y]}
\end{array}
$$

que mede o quanto o mapa $X \longrightarrow \nabla_{X}$ falha em ser homomorfismo de álgebras de Lie. Por definição, o comutador de elementos de uma álgebra de Lie, pertence a própria álgebra de 
Lie. A falha do comutador de uma distribuição em pertencer a distribuição é uma medida da sua falta de integrabilidade. Como veremos, uma falha da aplicação acima em ser um homomorfismo de álgebras de Lie em um dado ponto representa a falta de integrabilidade de qualquer referencial local paralelo (ou ainda, a impossibilidade de escolhermos um referencial que não observe os efeitos da curvatura).

Em termos mais pedestres, podemos dizer que o primeiro termo do lado direito de (1.53), o comutador, mede a diferença entre derivar covariantemente primeiro em uma direção e depois na outra, e tomar as derivadas na ordem inversa. Mas isso não diz muita coisa, já que mesmo em um fibrado trivial, com a conexão trivial, as derivadas covariantes podem não comutar simplesmente porque os campos vetoriais $X, Y$ podem ter o seu colchete de Lie não nulo. I.e.: para uma seção do fibrado trivial $f: M \rightarrow \mathbb{R}^{k}$, e para a derivada usual do $\mathbb{R}^{k}$, os primeiros termos equivalem à $(X Y-Y X) f$, mas não é necesariamente verdade que $(X Y-Y X) f=0$. O segundo termo corrige este efeito. Mostremos pois algumas características básicas do tensor de curvatura.

Theorem 5 Para todo $x \in M$ existe um mapa linear $\Omega(X, Y)_{x}: E_{p} \rightarrow E_{p}$ tal que se $s \in \Gamma(E)$ então

$$
(\Omega(X, Y) s)(x)=\Omega(X, Y)_{x} s(x)
$$

O enunciado do teorema equivale a dizer que $\Omega(X, Y) \in \operatorname{Diff}^{0}(E, E)$, ou seja, que $\Omega(X, Y)$, tem caráter tensorial, já que o resultado de sua aplicação só depende do valor dos campos no ponto de aplicação.

Dem: Nós temos que

$$
\left(\left[\nabla_{X}, \nabla_{Y}\right]-\nabla_{[X, Y]}\right)(f s)=f\left(\left[\nabla_{X}, \nabla_{Y}\right]-\nabla_{[X, Y]}\right) s
$$

Portanto se $f(x)=0$ nós temos: $(\Omega(X, Y) f s)(x)=0$ Provamos então que $\Omega(X, Y)$ é $C^{\infty}(M)$ linear, i.e.: tem caráter tensorial, ou ainda, pertence a $\operatorname{Diff}^{0}(E, E)$. Para provar que isso implica que só depende do valor no ponto basta tomarmos uma função que se anule em uma vizinhança arbitrária do dado ponto.

Theorem 6 Existe uma 2-forma $\Omega$ em $M$ com valores em $\operatorname{End}(E)$ (ou seja, $\Omega \in \Gamma\left(\Lambda^{2}(M) \otimes\right.$ $\operatorname{End}(E)$ ) tal que para todos $X, Y \in \Gamma(T M)$,

$$
\Omega_{x}\left(X_{x}, Y_{x}\right)=\Omega(X, Y)_{x}
$$

Dem: $\Omega(X, Y)$ é claramente anti-simétrica, vimos também que $\Omega(X, Y) \in \operatorname{End}(E)$, agora nos resta ver que $\Omega$ é $C^{\infty}$-linear na primeira entrada (i.e.: tem caráter tensorial e portanto só depende de seus valores no ponto). Calculando $\Omega(f X, Y)$ obtemos, depois de um pouco de álgebra, $\Omega(f X, Y)=f \Omega(X, Y)$.

\section{Estrutura do Espaço de Todas as Conexões em E}

Chamamos de $\mathcal{C}(E)$ o espaço de todas as conexões em $E$ e seja

$$
\Delta(E)=\Gamma\left(T^{*} M \otimes \operatorname{End}(E)\right)
$$


Definition 5 Se $\omega \in \Delta(E)$ e $s \in \Gamma(E)$, definimos $\omega(s) \in \Gamma\left(T^{*} M \otimes E\right)$ por

$$
\omega(s)(X)=\omega(X, s(p)) \in E_{x}
$$

onde $X \in T_{p} M$.

$\mathrm{Na}$ verdade estamos mudando o enfoque sobre $\omega$ de

$$
\Delta(E)=\Gamma\left(T^{*} M \otimes \operatorname{End}(E)\right) \rightarrow \Gamma\left(E^{*} \otimes\left(T^{*} M \otimes E\right)\right)=\operatorname{Diff}^{0}\left(E, T^{*} M \otimes E\right)
$$

Ou seja, existe um isomorfismo trivial entre os dois espaços que só muda a ordem de operação de seus elementos.

Theorem 7 Se $\nabla^{0} \in \mathcal{C}(E)$ e para todos $\omega \in \Delta(E)$ nós definirmos

$$
\begin{aligned}
\nabla^{\omega}: \Gamma(E) & \rightarrow \Gamma\left(T^{*} M \otimes E\right) \\
s & \mapsto \nabla^{0} s+\omega(s)
\end{aligned}
$$

Então $\nabla^{\omega} \in \mathcal{C}(E)$ e o mapa $\omega \mapsto \nabla^{\omega}$ é bijetor.

Dem: $\nabla^{\omega}$ é $\mathbb{R}$-linear já que ambos $\nabla^{0}$ e $\omega$ o são. Se $f \in C^{\infty}(M, \mathbb{R})$ e $s \in \Gamma(E)$, então temos

$$
\nabla^{\omega} f s=f \nabla^{0} s+d f \otimes s+f \omega(s)=f \nabla^{\omega} s+d f \otimes s
$$

Agora provemos que $\omega \mapsto \nabla^{\omega}$ é bijetor. Que é injetora é trivial. Seja então $\nabla^{\alpha} \in \mathcal{C}$, nós temos que:

$$
\begin{aligned}
& \nabla^{\alpha}(f s)=d f \otimes s+f \nabla^{\alpha} s \\
& \nabla^{0}(f s)=d f \otimes s+f \nabla^{0} s
\end{aligned}
$$

De (1.60) e (1.61) nós temos:

$$
\left(\nabla^{\alpha}-\nabla^{0}\right)(f s)=f\left(\nabla^{\alpha}-\nabla^{0}\right) s
$$

ou seja $\nabla^{\alpha}-\nabla^{0}$ é linear. I.e.:

$$
\nabla^{\alpha}-\nabla^{0} \in \operatorname{Diff}^{0}\left(E, T^{*} M \otimes E\right) \therefore \nabla^{\alpha}-\nabla^{0}=\omega
$$

para algum $\omega \in \Delta(E)$, então $\nabla^{\alpha}=\nabla^{0}+\omega$

Logo $\mathcal{C}(E)$ é um subespaço afim de $\operatorname{Diff}^{1}\left(E, T^{*} M \otimes E\right)$ e nós temos

$$
\mathcal{C}(E) \simeq \nabla^{0}+\operatorname{Diff}^{1}\left(E, T^{*} M \otimes E\right)
$$

Nós chamaremos de $\Delta(E)=\Gamma\left(T^{*} M \otimes \operatorname{End}(E)\right)$ o espaço das formas de conexão.

Já que a curvatura tem caráter tensorial, é interessante notar que faz sentido atribuir-la um valor zero em um dado ponto, independente do gauge, o que não podemos fazer com formas de conexão, que são afins. Uma forma de conexão não define por si só uma conexão $\nabla^{\omega}$, mas somente relativamente a outra conexão $\nabla^{0}$, que pode ser considerado a origem segundo a qual uma conexão é nula. Logo $\Delta(E)$ é o espaço das diferenças de conexão. Pela propriedade da trivialização local, podemos trabalhar localmente como se o fibrado fosse o produto (ou trivial) então é válido que estudemos conexões no fibrado trivial para depois globalizarmos algumas de suas propriedades. 


\section{Conexões em um Fibrado Trivial}

Seja $E$ o fibrado trivial em $M \times \mathbb{R}^{k}$, então $\Gamma(E)=\mathbb{C}^{\infty}\left(M, \mathbb{R}^{k}\right)$ e $\Gamma\left(\Lambda^{1}(M) \otimes E\right)$ é o espaço das formas a valores em $\mathbb{R}^{k}$. Como vimos, a escolha natural para $\nabla^{0}$ é $d$, a diferencial usual de uma função a valores vetoriais. Temos:

$$
\Delta(E)=\Gamma\left(T^{*} M \otimes \operatorname{End}(E)\right)=\Gamma\left(T^{*} M \otimes L\left(\mathbb{R}^{k}, \mathbb{R}^{k}\right)\right)
$$

então $\omega \rightarrow \nabla^{\omega}=d+\omega$ nos dá uma bijeção entre $\mathcal{C}(E)$ e formas a valores em $M(k \times k)$. Escrevamos então $\omega$ em termos de uma base de $\mathbb{R}^{k}, \mathcal{B}=\left\{e_{i}\right\}_{i=1}^{k}$ e tomamos a base dual $\mathcal{B}^{*}=\left\{e^{i}\right\}_{i=1}^{k}$, então é claro que $\left\{e_{i} \otimes e^{j}\right\}$ para $\{1 \leq i \leq k, 1 \leq j \leq k\}$ é a base associada de $L\left(\mathbb{R}^{k}, \mathbb{R}^{k}\right)$. Então, escrevendo $\omega$ nessa base, temos:

$$
\omega=\omega_{\alpha}^{\beta} \otimes e^{\alpha} \otimes e_{\beta}
$$

onde $\omega_{\alpha}^{\beta} \in \Gamma\left(T^{*} M\right)$. Agora seja $s=s^{i} e_{i} \in \Gamma(E)=\mathbb{C}^{\infty}\left(M, \mathbb{R}^{k}\right)$ então

$$
\nabla^{\omega} s=d\left(s^{i} e_{i}\right)+\omega\left(s^{i} e_{i}\right)=d s^{j} \otimes e_{j}+s^{i} \omega_{i}^{j} \otimes e_{j}
$$

Logo,

$$
\left(\nabla^{\omega} s\right)^{j}=d s^{j}+s^{i} \omega_{i}^{j}
$$

Logo se $X \in\left(T_{x} M\right)$,

$$
\nabla^{\omega} s(X)=\left(\nabla^{\omega} s\right)^{j}(X) e_{j} \Rightarrow\left(\nabla^{\omega} s\right)^{j}(X)=X\left[s^{j}\right]+s^{i}(x) \omega_{i}^{j}(X)
$$

Tomando $s=e_{k}$ temos

$$
\nabla^{\omega} s=\omega\left(e_{k}\right)=\omega_{k}^{\beta} \otimes e_{\beta} \Rightarrow\left(\nabla^{\omega} e_{k}\right)^{\beta}=\omega_{k}^{\beta}
$$

E finalmente obtemos :

$$
\nabla_{X}^{\omega} e_{k}=\omega_{k}^{\beta}(X) e_{\beta}
$$

Nós acabamos de provar que:

Theorem 8 Existe uma bijeção $\omega \rightarrow \nabla^{\omega}$ entre matrizes $k \times k$ de 1-formas $\omega$ sobre $M e$ conexões $\nabla^{\omega}$ no fibrado produto $E=M \times \mathbb{R}^{k}$. $\nabla^{\omega}$ é determinado por $\omega$ pela relação

$$
\left(\nabla_{v}^{\omega} s(x)\right)=v[s]+\omega(v) s(x)
$$

para $v \in T_{x} M$ e $s \in \Gamma(E)=C^{\infty}(M, \mathbb{R})$. Recíprocamente, $\nabla \in \mathcal{C}(E)$ determina $\omega$ tomando uma base de seções $\left\{e^{i}\right\}_{i=1}^{k}$ e expandindo a ação de $\nabla_{X}$ nessa base, para $X \in \Gamma(E)$ :

$$
\nabla_{X} e_{i}=\omega_{i}^{j}(X) e_{j}=\omega(X)\left(e_{i}\right)
$$

Então $\Omega \in \Gamma\left(\Lambda^{2}(M) \otimes L\left(\mathbb{R}^{k}, \mathbb{R}^{k}\right)\right.$ está relacionada a uma conexão $\omega$. Vejamos como.

$$
\begin{array}{r}
\Omega: \Gamma(T M \times T M) \rightarrow L\left(\mathbb{R}^{k}, \mathbb{R}^{k}\right) \\
(X, Y) \mapsto \Omega_{l}^{j}(X, Y) e^{l} \otimes e_{j}
\end{array}
$$


Onde $\Omega_{l}^{j}(X, Y)$ é uma matriz $k \times k$ de 2-formas em $M$. Logo, aplicando a $e_{i}$ ambos os lados da última equação:

$$
\Omega_{i}^{j}(X, Y) e_{j}=\Omega(X, Y)\left(e_{i}\right)=\left(\left[\nabla_{X}, \nabla_{Y}\right]-\nabla_{[X, Y]}\right) e_{i}
$$

onde lembramos o leitor que índices repetidos indicam uma somatória de termos. Mas

$$
\nabla_{X} e_{i}=\omega_{i}^{j}(X) e_{j} \Rightarrow \nabla_{Y}\left(\nabla_{X} e_{i}\right)=\left(Y\left[\omega_{i}^{j}(X)\right]+\omega_{i}^{k}(X) \omega_{k}^{j}(Y)\right) e_{j}
$$

Para calcularmos $\nabla_{X} \nabla_{Y} e_{i}$ basta invertermos $X \leftrightarrow Y$. Por último resta

$$
\nabla_{[X, Y]} e_{i}=\omega_{i}^{j}([X, Y]) e_{j}
$$

Juntando todos os termos obtemos:

$$
\begin{aligned}
\Omega_{i}^{j}(X, Y) e_{j} & =\left(X\left[\omega_{i}^{j}(Y)\right]+\omega_{i}^{k}(X) \omega_{k}^{j}(Y)-Y\left[\omega_{i}^{j}(X)\right]-\omega_{i}^{k}(Y) \omega_{k}^{j}(X)-\omega_{i}^{j}([X, Y])\right) e_{j} \\
& =d \omega_{i}^{j}(X, Y)+\omega_{i}^{k} \wedge \omega_{k}^{j}(X, Y)
\end{aligned}
$$

Portanto obtemos

$$
\Omega_{i}^{j}=d \omega_{i}^{j}+(\omega \wedge \omega)_{i}^{j}
$$

Onde $(\omega \wedge \omega)$ é a matriz de 2-formas resultante da multiplicação das matrizes de 1-forma $\omega$, onde a multiplicação de cada termo se dá com o produto exterior. Portanto, definindo $d \omega:=d \omega_{i}^{j} \otimes e^{i} \otimes e_{j}$ chegamos a

$$
\Omega=d \omega+\omega \wedge \omega
$$

Ou em palavras:

Theorem 9 Se $\omega$ é uma matriz $k \times k$ de 1-formas em $M$ e $\nabla=\nabla^{\omega}=d+\omega$ é a conexão correspondente no fibrado produto $E=M \times \mathbb{R}^{k}$, então a forma de curvatura $\Omega^{\omega}$ relacionada $a \nabla$ é a matriz $k \times k$ de 2-formas em $M$ dada por $\Omega^{\omega}=d \omega+\omega \wedge \omega$.

Esta forma de expressar $\Omega$ facilita em muito a derivação da Identidade de Bianchi:

$$
d \Omega^{\omega}+\omega \wedge \Omega^{\omega}-\Omega^{\omega} \wedge \omega=0
$$

Dem:

$$
\begin{aligned}
d \Omega & =d(d \omega)+d(\omega \wedge \omega) \\
& =d \omega \wedge \omega-\omega \wedge d \omega \\
& =(\Omega-\omega \wedge \omega) \wedge \omega-\omega \wedge(\Omega-\omega \wedge \omega) \\
& =\Omega \wedge \omega-\omega \wedge \Omega+\omega \wedge \omega \wedge \omega-\omega \wedge \omega \wedge \omega \\
& =\Omega \wedge \omega-(-1)^{2} \Omega \wedge \omega
\end{aligned}
$$

A identidade de Bianchi, é uma relação geométrica que, como veremos, representa leis de conservação. Claramente estes resultados se estendem naturalmente para uma dada trivialização local. Mas quanto dependem estes resultados das nossas escolhas de trivialização? 


\section{Mudança de Gauge}

Sejam $\left\{e_{i}\right\}_{i=1}^{k}$ e $\left\{\tilde{e}_{i}\right\}_{i=1}^{k}$ duas bases locais de $\Gamma\left(E_{\mid \theta}\right)$, relativas às trivializações $F=\psi^{-1}$ : $\theta \times \mathbb{R}^{k} \rightarrow \pi^{-1}(\theta)$ e $G=\phi^{-1}: \theta \times \mathbb{R}^{k} \rightarrow \pi^{-1}(\theta)$ respectivamente. E seja $g: \theta \rightarrow \Gamma\left(\operatorname{Aut}\left(\left.E\right|_{\theta}\right)\right)$ o mapa de transição de um ao outro. Então temos $\tilde{e}_{i}=g\left(e_{i}\right)$. Pela equação $(1.66), \omega(X) \in$ $\operatorname{End}\left(E_{x}\right)$, e poderíamos nos perguntar se $\omega$ como seção de transformações lineares escrita em outra seção de bases seria similar (no contexto de transformações lineares) à própria $\omega$. A resposta é negativa, pois aqui a própria transformação de bases varia sobre as fibras, e temos de levar esse efeito em conta ${ }^{7}$.

$$
\begin{aligned}
\widetilde{\omega}(X) \tilde{e}_{i} & =\nabla_{X} \tilde{e}_{i}=\nabla_{X}\left(g\left(e_{i}\right)\right)=d g(X)\left(e_{i}\right)+g\left(\nabla_{X} e_{i}\right)=d g(X)\left(e_{i}\right)+g \omega(X)\left(e_{i}\right) \\
& =(d g(X)+g \omega(X)) e_{i}=(d g(X)+g \omega(X)) g^{-1}\left(\tilde{e}_{i}\right)
\end{aligned}
$$

Portanto

$$
\widetilde{\omega}=(d g) g^{-1}+g \omega g^{-1}
$$

Calculemos então a mudança na forma de curvatura

$$
\widetilde{\Omega}=d \widetilde{\omega}+\widetilde{\omega} \wedge \widetilde{\omega}
$$

Nós temos que

$$
d \widetilde{\omega}=d\left((d g) g^{-1}\right)+d\left(g \omega g^{-1}\right)=-\left(d g \wedge g^{-1} d g g^{-1}\right)+d g \wedge \omega g^{-1}+g d \omega g^{-1}-g \omega \wedge g^{-1} d g g^{-1}
$$

e por outro lado

$$
\widetilde{\omega} \wedge \widetilde{\omega}=d g g^{-1} \wedge d g g^{-1}+d g \wedge \omega g^{-1}+g \omega g^{-1} \wedge d g g^{-1}+g \omega \wedge \omega g^{-1}
$$

Somando (1.74) e (1.75), obtemos

$$
\widetilde{\Omega}=g d \omega g^{-1}+g \omega \wedge \omega g^{-1}=g \Omega g^{-1}
$$

Logo se $\Omega(X, Y)$ for nula, ela será nula em todos os gauges, representando bem o seu caráter tensorial. Enquanto que para formas de conexão, mesmo que $\omega(X)=0$, nós temos de levar em conta o termo $d g g^{-1}$, que representa o caráter afim da forma de conexão (sem origem). Notemos que não derivamos o resultado usual de

$$
\widetilde{\omega}=g d g^{-1}+g \omega g^{-1}
$$

isso ocorre porque ao contrário da maioria das abordagens, aqui o grupo age sobre uma base à esquerda e não à direita. É fácil ver que utilizando a ação à direita recupera-se o resultado usual.

\footnotetext{
${ }^{7}$ Lembrando que em termos de bases locais o mapa de transição pode ser encarado como uma aplicação $g: \theta \rightarrow G L(k)$, poderíamos considerar o mapa de transição constante se um mesmo elemento de $G L(k)$ ligasse as bases sobre todos os pontos de $\theta$, mas sob outra trivialização isso não seria necessariamente verdade.
} 


\section{Abordagem Alternativa}

Esta seção pode ser ignorada sem prejuízo para a continuidade da exposição.

Em álgebra linear, nós sabemos que a ação de mudanças de base sobre transformações lineares é efetuada por conjugação por automorfismos, o que confere a esta conjugação também uma "interpretação passiva" (de significar a mesma transformação linear sob diferentes bases). A distinção entre tais conjugações "ativas" e "passivas" fica borrada no caso de transformações lineares porque não utilizamos em espaços vetoriais mudanças de base locais, os automorfismos são globais e portanto sua ação pode ser considerada uma nova transformação linear. No caso de variedades suaves a distinção entre transição de cartas e difeomorfismos globais é óbvia.

Tomando o ponto de vista ativo, ou global, aqui também podemos ter conexões "equivalentes", e assim como em transformações lineares, julgaremos duas conexões equivalentes se forem relacionadas pela congugação de automorfismos ( aqui um difeomorfismo $f: E \rightarrow E$ tal que $\pi \circ f=\pi$, onde $f$ leva $\pi^{-1}(x) \rightarrow \pi^{-1}(x)$ por um isomorfismo linear), i.e.: devemos também ter o mapa comutativo (para todo $X \in \Gamma(T M)$ ):

$$
\begin{array}{rll}
\Gamma(E) & \stackrel{\nabla_{X}}{\longrightarrow} & \Gamma(E) \\
g \downarrow & & \downarrow g \\
\Gamma(E) & \underset{\tilde{\nabla}_{X}}{\longrightarrow} & \Gamma(E)
\end{array}
$$

Exatamente como ocorre com a representação de transformações lineares sob isomorfismos lineares. I.e.: nós podemos representar a conexão $\nabla$ sob um isomorfismo de fibrados, e obteremos uma nova conexão

$$
\tilde{\nabla}_{X}(s)=g \nabla_{X}\left(g^{-1} s\right) \Rightarrow \widetilde{\nabla}_{X}=g \nabla_{X} g^{-1}
$$

ou ainda, $g \nabla_{X}=\widetilde{\nabla}_{X} g$. Chequemos pois que $\widetilde{\nabla}$ é realmente uma conexão:

(i) Se $s \in \Gamma(E), f \in C^{\infty}(M, \mathbb{R})$ e $X \in \Gamma(T M)$, então

$$
\tilde{\nabla}_{X}(f s)=g \nabla_{X}\left(g^{-1} f s\right)=g \nabla_{X}\left(f g^{-1} s\right)=g X[f] g^{-1} s+f g \nabla_{X}\left(g^{-1} s\right)=X[f] s+f \widetilde{\nabla}_{X}(s)
$$

(ii) O mapa $X \rightarrow \nabla_{X}$ é $C^{\infty}(M, \mathbb{R})$-linear:

$$
\tilde{\nabla}_{f X}=g \nabla_{f X} g^{-1}=f g \nabla_{X} g^{-1}=f \widetilde{\nabla}_{X}
$$

Agora podemos nos perguntar, como provamos que existe bijeção entre o espaço das conexões e o espaço das formas, se $\nabla$ estiver relacionado a $\omega$ e $\tilde{\nabla}$ estiver relacionado a $\widetilde{\omega}$ então qual é a relação entre $\omega$ e $\widetilde{\omega}$ ? Sobre $\pi^{-1}(\theta)$, o domínio de uma trivialização podemos colocar $\widetilde{\nabla}^{0}=d$, fazendo $g \nabla_{X}=\widetilde{\nabla}_{X} g$ : 


$$
\begin{aligned}
g \nabla_{X} e_{i} & =g \omega_{i}^{k}(X) e_{k}=\omega_{i}^{k}(X) g_{k}^{j} e_{j}=\left(\omega_{i}^{l}(X) g_{l}^{k}\right) e_{k} \\
\widetilde{\nabla}_{X}\left(g e_{i}\right) & =\widetilde{\nabla}_{X}\left(g_{i}^{j} e_{j}\right)=\left(d g_{i}^{k}+g_{i}^{j} \widetilde{\omega}_{j}^{k}(X)\right) e_{k} \\
& \therefore \quad d g_{i}^{k}+g_{i}^{j} \widetilde{\omega}_{j}^{k}(X)=\omega_{i}^{l}(X) g_{l}^{k}
\end{aligned}
$$

Válido para todo $X \in \Gamma\left(T M_{1} \theta\right)$, obtemos então:

$$
\widetilde{\omega}=(d g) g^{-1}+g \omega g^{-1}
$$

Para calcularmos a curvatura sob uma transformação de gauge, notemos que, a partir de (1.77), obtemos, para todos $X, Y \in \Gamma(T M)$ :

$$
\widetilde{\nabla}_{X} \widetilde{\nabla}_{Y}=g \nabla_{X} g^{-1} g \nabla_{Y} g^{-1}=g \nabla_{X} \nabla_{Y} g^{-1}
$$

e que

$$
\widetilde{\nabla}_{[X, Y]}=g \nabla_{[X, Y]} g^{-1}
$$

portanto obtemos:

$$
\widetilde{\Omega}=g \Omega g^{-1}
$$

E realmente, o efeito local de automorfismos globais é uma transformação de gauge. Vejamos pois outras formas de construir novas conexões a partir de antigas, construções necessárias para a introdução dos importantes conceitos de paralelismo e holonomia.

\subsection{Transporte Paralelo e Holonomia}

\section{Construindo Novas Conexões}

Proposition 5 Seja $\left\{\theta_{i}\right\}_{i \in I}$ uma cobertura de $M$ e $\nabla^{i}$ a conexão em $\pi^{-1}\left(\theta_{i}\right)$ de forma que $\nabla^{i}$ e $\nabla^{j}$ concordam em $E_{\mid \theta_{i} \cap \theta_{j}}$, para todos $i, j \in I$. Então existe uma única conexão $\nabla$ em E tal que $\nabla_{\mid \pi^{-1} \theta_{i}}=\nabla^{i}$

Dem: Que existe uma é fácil demonstrar usando partições da unidade associadas à cobertura $\left\{\theta_{i}\right\}$. Chamando essa partição de $\left\{\sigma_{i}\right\}$ e fazendo $\nabla=\sum_{i \in I} \sigma_{i} \nabla^{i}$ podemos facilmente verificar que obtemos a conexão desejada. Por outro lado, se houvesse duas diferentes, elas teriam de diferir em pelo menos um aberto (já que são lisas), podemos supor sem perda de generalidade que seriam então diferentes em um aberto contido em $E_{\mid \theta_{i}}$, logo não podem ambas ter restrição igual em $E_{\mid \theta_{i}}$.

Theorem $10 S e \nabla$ é qualquer conexão em $E$ existe uma conexão única $\nabla^{*}$ no fibrado dual $E^{*}$ tal que se $\sigma \in \Gamma\left(E^{*}\right)$ e $s \in \Gamma(E)$, então temos, para todo $X \in \Gamma(E)$ :

$$
X[\sigma(s)]=\nabla_{X}^{*} \sigma(s)+\sigma \nabla_{X} s
$$


Dem: A forma mais fácil de demonstrar esse fato é simplesmente tomando

$$
\nabla_{X}^{*} \sigma(s)=X[\sigma(s)]-\sigma \nabla_{X} s
$$

e mostrando que essa definição preenche os requisitos de uma conexão. Será útil no entanto achar a forma de conexão em termos de uma trivialização local, em termos de bases locais, $\left\{e_{i}\right\}$ e $\left\{e^{i}\right\}$ de $\Gamma\left(E_{\mid \theta_{i}}\right)$ e $\Gamma\left(E_{\mid \theta_{i}}^{*}\right)$ respectivamente. Então, chamando $\nabla_{X}^{*} e^{j}=\omega^{*}(X) e^{j}$, usando $\nabla_{X} e_{i}=\omega(X) e_{i}$ e a propriedade que a conexão comuta com a contração, i.e.: (1.82), obtemos:

$$
X\left[e^{j}\left(e_{i}\right)\right]=\left(\omega^{*}(X) e^{j}\right)\left(e_{i}\right)+e^{j} \omega(X) e_{i}=0 \Rightarrow\left(\omega^{*}(X) e^{j}\right)\left(e_{i}\right)=-e^{j}\left(\omega(X) e_{i}\right)
$$

Mas lembremos que se $S \in L\left(\mathbb{R}^{k}, \mathbb{R}^{k}\right)$, se $\lambda \in \Lambda^{1}\left(\mathbb{R}^{k}\right)$ e $v$ é um vetor em $\mathbb{R}^{k}$, então $\lambda(S v)=$ $S^{T} \lambda(v)$, i.e.: $\lambda S=S^{T} \lambda$, Onde o sobrescrito $T$ denota transposição da matriz. Então, utilizando o caso geral:

$$
-e^{j}\left(\omega(X) e_{i}\right)=\left(-\omega(X)^{T} e^{j}\right)\left(e_{i}\right)=\left(\omega^{*}(X) e^{j}\right)\left(e_{i}\right)
$$

Logo, como isso é válido para todos $i, j, X$ obtemos, $\omega^{*}=-\omega^{T}$. Ou ainda, sendo $\left\{\lambda_{i}\right\}$ uma base de campos para $\Gamma\left(E_{\mid \theta_{i}}^{*}\right)$ dual a $\left\{e_{i}\right\}$, obtemos facilmente

$$
\begin{gathered}
\nabla_{X}^{*} \lambda_{i}\left(e_{j}\right)+\lambda_{i}\left(\nabla_{X} e_{j}\right)=\omega_{i}^{* k}(X) \lambda_{k}\left(e_{j}\right)+\lambda_{i} \omega_{j}^{k}(X) e_{k}=0 \\
\therefore \omega_{i}^{* j}=-\omega_{j}^{i}
\end{gathered}
$$

Demos a primeira demonstração porque a segunda contraria um pouco a notação e prática dos físicos, de não considerar formas duais como campos vetoriais, e de denotá-las por índices superiores ao invés de inferiores. Considerando formas simplesmente como seções de um fibrado vetorial (e não somente como duais de seções) a demonstração se torna trivial.

Theorem $11 S e \nabla^{i}$ é conexão em um fibrado $E^{i}$ sobre $M, i=1,2$, então existe uma única $\nabla=\nabla^{1} \otimes 1+1 \otimes \nabla^{2}$ em $E^{1} \otimes E^{2}$, tal qual se $s^{i} \in \Gamma\left(E^{i}\right)$ e $X \in \Gamma(T M)$ Então

$$
\nabla_{X}\left(s^{1} \otimes s^{2}\right)=\nabla_{X}^{1} s^{1} \otimes s^{2}+s^{1} \otimes \nabla_{X}^{2} s^{2}
$$

Dem: Novamente tomamos duas bases $\left\{e_{i}\right\}_{i=1}^{k},\left\{b_{i}\right\}_{i=1}^{l}$ sobre $\left.E^{1}\right|_{\theta}$ e $\left.E^{2}\right|_{\theta}$ respectivamente. Então temos, se $\nabla$ satisfaz (1.85):

$$
\begin{gathered}
\nabla_{X}\left(e_{i} \otimes b_{j}\right)=\nabla_{X}^{1} e_{i} \otimes b_{j}+e_{i} \otimes \nabla_{X}^{2} b_{j}= \\
\sum_{m=1}^{k} \omega^{(1)}\left(e_{i}\right) \otimes b_{j}+\sum_{p=1}^{l} e_{i} \otimes \omega^{(2)}\left(b_{j}\right)
\end{gathered}
$$

Logo como $\left\{e_{m} \otimes b_{j}\right\}_{i=1, j=1}^{i=k, j=l}$ é base de $\left.\left(E^{1} \otimes E^{2}\right)\right|_{\theta}$, para que $\nabla$ obedeça (1.85), e já que $\omega^{1}$ e $\omega^{2}$ são únicos para $\nabla^{1}$ e $\nabla^{2}, \omega$ é única e tem a forma

$$
\omega=\omega^{1} \otimes I d+I d \otimes \omega^{2}
$$


Agora, do fibrado $(E, \pi, M)$, e dado um mapa $\phi: N \rightarrow M$, nós obtemos o fibrado do pull-back $\phi^{*}(E)$ sobre $N$ tomando como fibra sobre $x \in N$,

$$
\left(\phi^{*}(E)\right)_{x} \simeq\left(E_{\phi(x)}\right)
$$

i.e.: nós puxamos as fibras juntamente com os pontos de $\phi(N)$. Logo nós obtemos o mapa linear

$$
\begin{aligned}
\phi^{*}: \Gamma(E) & \longrightarrow \Gamma\left(\phi^{*}(E)\right) \\
s & \longmapsto s \circ \phi
\end{aligned}
$$

Theorem 12 Dado $\nabla \in \mathcal{C}(E)$, existe uma única conexão $\nabla^{\phi}$ para $\phi^{*}(E)$ tal que se $s \in \Gamma(E)$, $Y \in \Gamma(T N)$ e $X=d \phi(Y)$ então

$$
\nabla_{Y}^{\phi}\left(\phi^{*} s\right)=\phi^{*}\left(\nabla_{X} s\right)
$$

Dem: Novamente escolhemos uma base $\left\{e_{i}\right\}_{i=1}^{k}$ em $\left.E\right|_{\theta}$, e obtemos

$$
\left.\phi^{*}\left(\nabla_{X} e_{i}\right)=\phi^{*}\left(\omega_{i}^{j}(X) e_{j}\right)=\omega_{i}^{j}(d \phi(Y)) \phi^{*} e_{j}\right)
$$

É claro que $\left\{\phi^{*}\left(e_{i}\right)\right\}_{i=1}^{k}=\left\{\tilde{e}_{i}\right\}_{i=1}^{k}$ é base de $\Gamma\left(\phi^{*}(E)\right)$ então

$$
\phi^{*}\left(\nabla_{X} e_{i}\right)=\omega_{i}^{j}(d \phi(Y)) \tilde{e_{j}} \Rightarrow \tilde{\omega}_{i}^{j}=\phi^{*} \omega_{i}^{j}
$$

Portanto temos

$$
\left(\nabla^{\phi} \phi^{*} e_{i}\right)^{j}=\phi^{*} \omega_{i}^{j}
$$

\section{Exemplos}

- Se $N$ for subvariedade de $M$ e $\imath: N \hookrightarrow M$ então $\imath^{*}(E)=E_{\mid N}$. É claro que se $\theta \subset \imath(N)$ é domínio de um gauge, $X \in \Gamma\left(T M_{\mid \theta}\right)$ e $s^{1}, s^{2} \in \Gamma(E)$ tal que se $s_{\mid \theta}^{1}=s_{\mid \theta}^{2}$, então $\nabla_{X} s^{1}=\nabla_{X} s^{2}$

- Se $N=I=[a, b]$ e $\phi=\sigma: I \longrightarrow M$, temos $\sigma^{*}(s)_{t}=s(\sigma(t))$ e escrevemos para $\nabla^{\sigma} \in \mathcal{C}\left(\sigma^{*}(E)\right)$ e $Y \in \Gamma(T I)$

$$
\nabla_{Y}^{\sigma} \sigma^{*} s=\nabla_{\frac{\partial}{\partial t}}^{\sigma} \sigma^{*} s=\frac{D}{d t}(s \circ \sigma)=\nabla_{\sigma^{\prime}} s
$$

chamada de derivada covariante ao longo de $\sigma$. Novamente tomando $s=e_{i}$, por (1.90) nós temos

$$
\left(\nabla_{\frac{\partial}{\partial t}}^{\sigma} \sigma^{*}\left(e_{i}\right)\right)^{j}=\omega_{i}^{j}\left(d \sigma\left(\frac{\partial}{\partial t}\right)\right)=\omega_{i}^{j}\left(\sigma^{\prime}\right)=\left(\nabla_{\sigma^{\prime}} e_{i}\right)^{j}
$$

Portanto, como vimos: $(\nabla s)^{j}=d s^{j}+s^{i} \omega_{i}^{j}$ e obtemos

$$
\left(\nabla_{\frac{\partial}{\partial t}}^{\sigma} \sigma^{*} s\right)^{j}=d s^{j}\left(\frac{\partial}{\partial t}\right)+s^{i} \omega_{i}^{j}\left(\sigma^{\prime}\right)=\frac{d s^{j}}{d t}+s^{i} \omega_{i}^{j}\left(\sigma^{\prime}\right)
$$

se $\left\{x^{i}\right\}_{i=1}^{n}$ são coordenadas locais de $M$ escrevemos $\omega_{i}^{j}=\Gamma_{i k}^{j} d x^{k}$ e portanto

$$
\frac{D s^{j}}{d t}=\frac{d s^{j}}{d t}+\Gamma_{i k}^{j} \frac{d \sigma^{k}}{d t} s^{i}
$$

Note-se que essa equação é uma equação diferencial ordinária linear de primeira ordem, com coeficientes lisos na função vetorial $\left(s^{i}(t), \cdots, s^{k}(t)\right) \in \mathbb{R}^{k}$. 


\section{Transporte Paralelo}

Como mencionamos $\nabla$ é chamada de flat ou plana, se e somente se $\Omega^{\nabla}=0$. Como $\nabla$ é uma 2-forma, se $\operatorname{dim} M=1, \Omega \equiv 0$. Logo, se $\sigma: I=[0,1] \rightarrow M$ e $\nabla \in \mathcal{C}(E)$, então $\nabla^{\sigma}$ é flat. Logo para cada tal $\nabla$ existe uma forma canônica de comparar vetores ao longo de $\sigma$.

\section{Definition 6 Seja}

$$
\frac{D}{d t}=\nabla_{\sigma^{\prime}}^{\sigma}: \Gamma\left(\sigma^{*}(E)\right) \rightarrow \Gamma\left(\sigma^{*}(E)\right)
$$

que é linear. Chamamos $P(\sigma):=K \operatorname{Ker} \nabla_{\sigma^{\prime}}^{\sigma}$, o espaço de campos paralelos ao longo de $\sigma$.

Theorem 13 mapa

$$
\begin{aligned}
\sigma_{t}: P(\sigma) & \longrightarrow E_{\sigma(t)} \\
s & \longmapsto s(t)
\end{aligned}
$$

é um isomorfismo linear.

Dem: Pelo teorema de existência e unicidade de soluções, dado $v \in E_{\mid \sigma\left(t_{0}\right)}$ qualquer, existe uma única solução para (1.94) (i.e.: existe uma única seção $s \in \Gamma\left(\sigma^{*} E\right)$ ) que satisfaz $s\left(t_{0}\right)=v$ e $\nabla_{\sigma^{\prime}}^{\sigma} s(t)=0$ para todo $t \in[0,1]$, ou seja, existe um único elemento associado de $P(\sigma)$. Além disso, a equação diferencial é linear, portanto depende linearmente de seus parâmetros iniciais. Portanto o mapa $\sigma_{t}$ que leva o espaço vetorial $P(\sigma)$ no espaço vetorial $E_{\sigma(t)}$ é uma bijeção linear.

Definition 7 Para $t_{1}, t_{2} \in I$ definimos o isomorfismo linear (chamado de transporte paralelo):

$$
\begin{aligned}
P_{\sigma}\left(t_{1}, t_{2}\right): E_{\sigma\left(t_{1}\right)} & \rightarrow E_{\sigma\left(t_{2}\right)} \\
v & \longmapsto s\left(t_{2}\right)
\end{aligned}
$$

onde $s \in P(\sigma) \subset \Gamma\left(\sigma^{*}(E)\right)$ é dado por $s=\left(\sigma_{t_{1}}\right)^{-1}(v)$.

Valem as seguintes propriedades:

(i) $P_{\sigma}(t, t)=I d_{E_{\sigma(t)}}$

(ii) $P_{\sigma}\left(t_{3}, t_{2}\right) P_{\sigma}\left(t_{2}, t_{1}\right)=P_{\sigma}\left(t_{3}, t_{1}\right)$ já que se $v=s\left(t_{1}\right)$, como a solução é única: $P_{\sigma}\left(t_{3}, t_{2}\right) P_{\sigma}\left(t_{2}, t_{1}\right)$ : $s\left(t_{1}\right) \mapsto s\left(t_{2}\right) \mapsto s\left(t_{3}\right)=P_{\sigma}\left(t_{3}, t_{1}\right) s\left(t_{1}\right)$

(iii) Dos itens (i) e (ii) obtemos facilmente que $P_{\sigma}\left(t_{1}, t_{2}\right)=P_{\sigma}\left(t_{2}, t_{1}\right)^{-1}$.

Como dissemos, dado $\nabla$ podemos comparar vetores de forma canônica ao longo de qualquer curva, e o transporte paralelo fornece o método geométrico de manter campos "constantes" sob essa comparação. Notemos que a mesma construção não vale para uma imersão $\phi: N \rightarrow$ $M$ se $\nabla^{\phi}$ não for plana, já que se fosse possível poderíamos achar uma trivialização local de $\phi^{*}(E)$ onde $\nabla^{\phi}=d$ e portanto $\Omega^{\phi}=0$, o que é contraditório. Veremos mais adiante que isto está relacionado com a integrabilidade de bases de campos paralelos ( a própria equação (1.53) aponta para o Teorema de Frobenius em termos de formas.) 
Proposition 6 A conexão $\nabla$ pode ser recuperada do transporte paralelo.

Dem: Dado $s \in \Gamma(E)$ e $X \in \Gamma(T M)$, tomamos $\sigma:[0,1] \rightarrow M$ qualquer tal que $\sigma^{\prime}(0)=X_{\sigma(0)}$. Nós temos que, seja $\left\{e_{i}(0)\right\}_{i=1}^{k}$ base de $E_{\sigma(0)}$, tomamos, para todo $i=1 \cdots k$

$$
e_{i}(t)=P_{\sigma}(t, 0) e_{i}(0)
$$

Como $P_{\sigma}(t, 0)$ é isomorfismo linear, $\left\{e_{i}(t)\right\}_{i=1}^{k}$ é base de $E_{\sigma(t)}$. Logo podemos escrever

$$
s(\sigma(t))=s^{i}(\sigma(t)) e_{i}(t)
$$

e usando a regra de Leibnitz:

$$
\nabla_{\sigma^{\prime}(0)} s=\frac{D}{d t}\left(s(\sigma(t))_{\mid t=0}=\left.\frac{d}{d t}\right|_{\mid t=0}\left(s^{i}(\sigma(t))\right) e_{i}(0)\right.
$$

Definimos então a curva em $E_{\sigma(0)}$ :

$$
\begin{gathered}
\tilde{s}: I \rightarrow E_{\sigma(0)} \\
t \longmapsto P_{\sigma}(0, t) s(\sigma(t))
\end{gathered}
$$

Mas

$$
\dot{P}_{\sigma}(0, t) s(\sigma(t))=P_{\sigma}(0, t)\left(s^{i}(\sigma(t)) e_{i}(t)\right)=s^{i}(\sigma(t)) e_{i}(0)
$$

Portanto chegamos a

$$
\left(\nabla_{X} s\right)_{\sigma(0)}=\nabla_{\sigma^{\prime}(0)} s=\left.\frac{d}{d t}\right|_{t=0} \tilde{s}(t)
$$

Portanto podemos considerar a derivada covariante como a forma infinitesimal do transporte paralelo, isto é como o mecanismo operacional do conceito geométrico de transporte paralelo levado ao limite.

Sejam $\left\{e_{i}\right\}_{i=1}^{k}$ e $\left\{\tilde{e}_{i}\right\}_{i=1}^{k}$ bases locais sobre $\sigma^{*}(E)$ e $\omega$ e $\widetilde{\omega}$ as respectivas formas de conexão. Se $g:[0,1] \rightarrow G L(k)$ é função de transição de gauge, nós sabemos que $\omega=g \widetilde{\omega} g^{-1}+d g^{-1} g$, agora se a base local $\left\{\tilde{e}_{i}\right\}_{i=1}^{k}$ é tal que

$$
\tilde{e_{i}}(t)=P_{\sigma}(t, 0) \tilde{e_{i}}(0) \Rightarrow \nabla^{\sigma} \tilde{e}_{i}=0 \Rightarrow \widetilde{\omega}=0
$$

Logo obtemos $\nabla^{\sigma}=d$ na base $\left\{\tilde{e}_{i}\right\}_{i=1}^{k}$ e $\nabla^{\sigma}=d g^{-1} g$ na base $\left\{e_{i}\right\}_{i=1}^{k}$. Note-se que não mudamos a base $\left\{e_{i}\right\}_{i=1}^{k} \mathrm{em} \mathrm{si}$, mas as novas funções de transição absorvem o efeito do antigo $\widetilde{\omega}$.

\section{Holonomia}

Sejam $\alpha$ e $\beta$ dois caminhos contínuos tal que $\alpha(0)=p$ e $\alpha(1)=x=\beta(0)$ e $\beta(1)=q$. Definimos os caminhos $\alpha^{-1}$ e $\beta$ o $\alpha$ por $\alpha^{-1}(t)=\alpha(1-t)$ e por

$$
\beta \circ \alpha(t)= \begin{cases}\alpha(2 t) & \text { se } 0 \leq t \leq 1 / 2 \\ \beta(2 t-1) & \text { se } 1 / 2 \leq t \leq 1\end{cases}
$$


Logo, pela propriedade (2) do transporte paralelo:

$$
\begin{gathered}
P_{\beta \alpha}(1,0)=P_{\beta \alpha}\left(1, \frac{1}{2}\right) \circ P_{\beta \alpha}\left(\frac{1}{2}, 0\right)=P_{\beta}(1,0) \circ P_{\alpha}(1.0) \\
P_{\alpha^{-1}}(1,0) \circ P_{\alpha}(1,0)=I d \Rightarrow P_{\alpha}(1,0)^{-1}=P_{\alpha^{-1}}(1,0)
\end{gathered}
$$

Agora seja

$$
\Lambda_{x}:=\{\sigma:[0,1] \rightarrow M \mid \sigma(0)=x=\sigma(1)\}
$$

Nós temos que, abreviando a notação, $P_{\sigma}(1,0):=P_{\sigma}: E_{x} \rightarrow E_{x}$ é isomorfismo linear, logo $P_{\sigma} \in G L\left(E_{x}\right)$. Definimos então o grupo de holonomia de $\nabla$ no ponto :

$$
\operatorname{Hol}_{x}(\nabla):=\left\{P_{\sigma}: \sigma \in \Lambda_{x}\right\} \subset G L\left(E_{x}\right)
$$

$\operatorname{Hol}_{x}(\nabla)$ é realmente um subgrupo de $G L\left(E_{x}\right)$ já que por (1.99), se $\alpha, \beta \in \Lambda_{x}$ então para $P_{\alpha}, P_{\beta} \in \operatorname{Hol}_{x}(\nabla)$ nós temos que $P_{\alpha} \circ P_{\beta}=P_{\alpha \beta} \in \operatorname{Hol}_{x}(\nabla)$ e os termos contém inversa por (1.100), já que se $\alpha \in \Lambda_{x} \Rightarrow \alpha^{-1} \in \Lambda_{x}$. Em outras palavras, o mapa $\sigma \rightarrow P_{\sigma}$ é um homomorfismo de grupos entre $\Lambda_{x}$ e um subgrupo de $G L\left(E_{x}\right)$. Supondo que $M$ seja conexo, dados $x, y \in M$, existe curva $\gamma:[0,1] \rightarrow M$ tal que $\gamma(0)=x$ e $\gamma(1)=y$, e $P_{\gamma}(1,0): E_{x} \rightarrow E_{y}$ é isomorfismo linear. Logo, como se $\alpha \in \Lambda_{x}$ então $\gamma \circ \alpha \circ \gamma^{-1} \in \Lambda_{y}$ e $P_{\gamma \alpha \gamma^{-1}}=P_{\gamma} \circ P_{\alpha} \circ P_{\gamma}^{-1}$ , então existe uma conjugação entre $\operatorname{Hol}_{x}(\nabla)$ e $\operatorname{Hol}_{y}(\nabla)$. I.e.:

$$
\operatorname{Hol}_{y}(\nabla)=P_{\gamma} \circ \operatorname{Hol}_{x}(\nabla) \circ P_{\gamma}^{-1}
$$

Isso mostra que o grupo de holonomia $\mathrm{Hol}_{x}(\nabla)$ independe do ponto base no seguinte sentido: suponha que $E$ tenha fibra isomorfa a $\mathbb{R}^{k}$, então qualquer identificação $E_{x} \simeq \mathbb{R}^{k}$ induz um isomorfismo entre o grupo das transformações lineares inversíveis de $E_{x}$ com o grupo das matrizes inversíveis $G L\left(E_{x}\right) \simeq G L(k, \mathbb{R})$. Então identificamos $\operatorname{Hol}_{x}(\nabla)=H_{x}(\nabla)<$ $G L(k, \mathbb{R})$. Mas se escolhermos outra base para $E_{x}$, i.e.: outra identificação, nós temos de aplicar uma transformação de semelhança em todos os elementos de $H_{x}(\nabla)$, ou seja, obtemos o subgrupo $a H_{x}(\nabla) a^{-1}$ onde $a \in G L(k, \mathbb{R})$ é a transformação entre as bases. Obtemos que o grupo de holonomia está bem definido como subgrupo de $\mathbf{G L}(k), \mathbb{R}$ a menos de conjugação.

Proposition 7 Seja $(E, \pi, M)$ fibrado vetorial com fibra típica $\mathbb{R}^{k}, \nabla \in \mathcal{C}(E)$ e $M$ simplesmente conexo.Então $\operatorname{Hol}(\nabla)$ é um subgrupo de Lie de $G L(k, \mathbb{R})$ conexo.

Dem: Seja $\gamma \in \Lambda_{x}$ e $F(s, t)$ uma homotopia entre $\gamma$ e o laço constante. Então $s \mapsto P_{F_{s}}(1,0)$ é um mapa contínuo ${ }^{8}$ entre $[0,1]$ e $\operatorname{Hol}(\nabla)$. Como $P_{F_{0}}(1,0)=I d$ e $P_{F_{1}}(1,0)=P_{\gamma}(1,0)$, cada $P_{\gamma}(1,0)$ pode ser ligado a identidade por um caminho contínuoem $\mathrm{Hol}(\nabla)$. Por um teorema de Yamabe [?] todo subgrupo conexo por caminhos de um grupo de Lie é um subgrupo de Lie conexo.

\section{Definition $8 \operatorname{Hol}^{0}(\nabla):=\left\{P_{\gamma}: \gamma\right.$ é homotópica a um ponto $\}$.}

\footnotetext{
${ }^{8}$ Os teroremas de soluções de E.D.O.s garantem que as soluções dependentes de parâmetros, dependem continuamente de seus parâmetros, bem como de suas condições iniciais.
} 
Theorem 14 Se $\sigma$ é homotópica a um ponto, então a conexão $\nabla$ é flat se e somente se $P_{\sigma}=I d$

\section{Dem:}

Sejam $\gamma_{0}$ e $\gamma_{1}$ homotópicas, ou seja, existe função contínua:

$$
\begin{array}{r}
F:[0,1] \times[0,1] \rightarrow M \\
(s, t) \longmapsto F(s, t)
\end{array}
$$

tal que, para $s$ fixo: $F(s, t)=F_{s}(t)=\gamma_{s}(t)$ família contínua de curvas interpolando $\gamma_{1}$ e $\gamma_{0}$. Além disso $\gamma_{s}(0)=x$ e $\gamma_{s}(1)=y$. Agora sejam

$$
{\frac{\partial}{\partial s} \mid\left(s_{0}, t_{0}\right)}_{:=F_{*} \frac{d}{d s \mid\left(s_{0}, t_{0}\right)}} ; \quad{\left.\frac{\partial}{\partial t}\right|_{\mid\left(s_{0}, t_{0}\right)}:=F_{*} \frac{d}{d t \mid\left(s_{0}, t_{0}\right)}}
$$

E seja $v \in E_{x}$. Definimos $X \in \Gamma\left(F^{*} E\right)$ por $X_{(F(s, t))}:=P_{\gamma_{s}}(t, 0) v$. Obviamente, para todo $s \in[0,1], X_{(F(s, 0))}=v$, portanto

$$
\left(\nabla_{\frac{\partial}{\partial s}} X\right)_{\mid\left(s_{0}, 0\right)}=0
$$

para todo $s_{0} \in[0,1]$. Agora, como a curvatura é nula:

$$
R\left(\frac{\partial}{\partial s}, \frac{\partial}{\partial t}\right) X=\nabla_{\frac{\partial}{\partial t}} \nabla_{\frac{\partial}{\partial s}} X-\nabla_{\frac{\partial}{\partial s}} \nabla_{\frac{\partial}{\partial t}} X=0
$$

Já que, como $(s, t)$ são coordenadas da parametrização $F(s, t)$ :

$$
\left[\frac{\partial}{\partial s}, \frac{\partial}{\partial t}\right]=F_{*}\left[\frac{d}{d s}, \frac{d}{d t}\right]=0
$$

Agora, pela definição de $X$, nós temos $\left(\nabla_{\frac{\partial}{\partial t}} X\right)_{\mid\left(s_{0}, t_{0}\right)}=0$, para todos $s_{0}, t_{0}$. Então, usando (1.103)

$$
\nabla_{\frac{\partial}{\partial t}} \nabla_{\frac{\partial}{\partial s}} X=0
$$

Ora, mas isso quer dizer que para cada $s_{0} \in[0,1]$ o campo $\left(\nabla_{\frac{\partial}{\partial s}} X\right)$ é transportado paralelamente ao longo de $\gamma_{s}$, i.e.:

$$
\left(\nabla_{\frac{\partial}{\partial s}} X\right)_{\mid\left(s_{0}, t_{0}\right)}=P_{\gamma_{s}}\left(t_{0}, 0\right)\left(\left(\nabla_{\frac{\partial}{\partial t}} X\right)_{\mid\left(s_{0}, 0\right)}\right)
$$

Então nós temos por (1.102) que (1.105) será o transporte paralelo de um vetor nulo, logo obtemos que

$$
\left(\nabla_{\frac{\partial}{\partial s}} X\right)_{\mid\left(s_{0}, t_{0}\right)}=0
$$

Portanto, em particular, nós que $\left(\nabla_{\frac{\partial}{\partial s}} X\right)_{\mid\left(s_{0}, 1\right)}=0$. Ou seja, a curva sobre a fibra $E_{y}$, $X(s, 1) \in E_{y}$ é uma curva constante, independe de $s \in[0,1]$. Logo o transporte paralelo de conexões flat é invariante por curvas homotópicas. Isso significa que em um domínio 
simplesmente conexo, se tivermos uma conexão flat, podemos estabelecer uma base de vetores paralelos, já que em cada ponto $y$ a base paralelamente transportada desde o ponto $x$ não dependerá da curva que liga os dois pontos.

Provemos pois alguns teoremas úteis sobre grupos e álgebras de holonomia.

Proposition $8 \mathrm{Hol}^{\mathrm{P}}(\nabla)$ é um subgrupo de Lie conexo de $G L(k, \mathbb{R})$, é a componente conexa de $\operatorname{Hol}(\nabla)$ que contém a identidade e é um subgrupo normal de $\operatorname{Hol}(\nabla)$. Além disso, existe um homeomorfismo de grupos sobrejetivo natural

$$
\Phi: \pi_{1}(M) \rightarrow \operatorname{Hol}(\nabla) /\left(\operatorname{Hol}^{0}(\nabla)\right)
$$

Demonstração: Pela proposição anterior, $\operatorname{Hol}^{0}(\nabla)$ é um subgrupo de Lie conexo. Sejam $\alpha, \gamma \in \Lambda_{p}, \gamma$ homotópico a $p$. Então $\alpha \circ \gamma \circ \alpha^{-1}$ também é homotópico a $p$. Portanto, $P_{\alpha \gamma \alpha^{-1}}=P_{\alpha} \circ P_{\gamma} \circ P_{\alpha}^{-1} \in \operatorname{Hol}_{p}^{0}(\nabla)$, sendo que $P_{\alpha} \in \operatorname{Hol}_{p}(\nabla), \operatorname{logo} \operatorname{Hol}_{p}^{0}(\nabla)$ é normal. Seja

$$
\begin{gathered}
\Phi: \pi_{1}(M) \rightarrow \operatorname{Hol}_{x}(\nabla) / \mathrm{Hol}_{x}^{0}(\nabla) \\
{[\gamma] \mapsto\left[P_{\gamma}\right]}
\end{gathered}
$$

Mostramos que é bem definido, isto é, que $\alpha, \gamma \in[\gamma]$, isto é:

$$
\alpha \simeq \gamma \Rightarrow\left[P_{\alpha}\right]=\left[P_{\gamma}\right]
$$

ou seja, que existe $a \in \operatorname{Hol}_{x}^{0}(\nabla)$, tal que $P_{\alpha}=P_{\gamma} \circ a$. Já que $\alpha \simeq \gamma$, então $\gamma^{-1} \alpha \simeq I d$. Portanto, $P_{\gamma^{-1} \alpha}=P_{\gamma^{-1}} \circ P_{\alpha}=a^{\prime} \in \mathrm{Hol}_{x}^{0} \Rightarrow P_{\alpha}=P_{\gamma} \circ a$, é claro por sua definição que $\Phi$ é um homo sobrejetor (epimorfismo). Como $\pi_{1}(M)$ é enumerável, o grupo quociente $\operatorname{Hol}_{x}(\nabla) / \operatorname{Hol}_{x}^{0}(\nabla)$ também o é, logo $\mathrm{Hol}_{x}(\nabla)$ é um grupo de Lie e $\mathrm{Hol}_{x}^{0}(\nabla)$ é a componente conexa de $\mathrm{Hol}_{x}(\nabla)$, que contém a identidade.

Definimos a álgebra de holonomia hol $(\nabla)$ como sendo a álgebra de $\operatorname{Hol}^{0}(\nabla)$. É uma subálgebra de $\mathfrak{g l}(k, \mathbb{R})$, definido a menos de conjugação (a ação adjunta de $\mathbf{G L}(k, \mathbb{R})$ ), já que $\operatorname{Hol}^{0}(\nabla)$ é um subgrupo de $\mathbf{G L}(k, \mathbb{R})$ definido a menos de conjugação. Da mesma forma $\mathfrak{h o l}_{x}(\nabla)$ é a álgebra de $\operatorname{Hol}_{x}^{0}(\nabla)$ (que é um subgrupo de $\mathbf{G L}\left(E_{x}\right)$ ). Logo, é uma subálgebra de $\operatorname{End}\left(E_{x}\right)$. Mesmo sendo $\mathrm{Hol}^{0}(\nabla)$ um subgrupo de Lie de $\mathbf{G L}(k, \mathbb{R})$, não é necessariamente um subgrupo fechado de $\mathbf{G L}(k, \mathbb{R})$, e mesmo que o seja, $\mathrm{Hol}(\nabla)$ não o é necessariamente.

\subsection{Conexões Admissíveis e Derivada de Gauge}

\section{Conexões Admissíveis em um G-fibrado}

Estudaremos agora conexões que de uma forma ou de outra são compatíveis com estruturas em $E$, significando que o transporte paralelo manterá as propriedades que caracterizam essas estruturas.

Seja $(E, \pi, M)$ um $G$-fibrado vetorial sobre $M$. Dada uma trivialização local, chamaremos de referencial admissível áquele induzido pela base canônica de $\mathbb{R}^{k}$ através desta trivialização. Nós sabemos que as funções de transição das trivializações estão, sobre todo ponto $x \in M$, dentro do grupo $G$, portanto, referenciais admissíveis para $E$ são ligados por representações de $G$, i.e.: sobre cada $x \in M$ as bases são ligadas por um único $g \in G$. 
Por outro lado, dado um mapa de transição de gauge qualquer $\sigma: \theta \rightarrow \rho(G) \subset G L(k)$ e uma trivialização local $\psi: \pi^{-1} \theta \rightarrow \theta \times \mathbb{R}^{k}$, nós vimos que $\tilde{\psi}$ dado por

$$
\psi \circ \tilde{\psi}^{-1}(x, u)=(x, \sigma(x) u)
$$

também é trivialização local do $G$-fibrado. Logo se $\{e\}$ é referencial admissível sobre $\theta$ dado por $\psi$, existe um único referencial admissível $\{\tilde{e}\}$ dado por $\tilde{\psi}$. Logo dadas duas bases admissíveis $\left\{e_{i}(x)\right\}_{i=1}^{k}$ e $\left\{\tilde{e}_{i}(x)\right\}_{i=1}^{k}$ sobre $x$, existe um único $\sigma(x)=g \in G$ tal que ${ }^{9}\left\{e_{i}\right\}_{i=1}^{k}=$ $\left\{g \tilde{e}_{i}\right\}_{i=1}^{k}$. Logo, uma vez escolhida uma base inicial em uma fibra, existe uma bijeção (que claramente não é canônica) entre as bases admissíveis e o grupo $G$.

Por exemplo, dado uma métrica sobre $M$, o fibrado das bases ortonormais sobre $M$ corresponde ao $O(n)$-fibrado vetorial. Esta construção será utilizada quando introduzirmos fibrados principais e os relacionarmos a fibrados vetoriais.

Um mapa linear $T \in L\left(E_{x}, E_{y}\right)$ é chamado de $G$-mapa se leva base admissível em base admissível, ou seja, se dadas base admissíveis em $x$ e $y$, a matriz de $T$ está em $G$. Denotaremos o conjunto de tais mapas por $L_{G}\left(E_{x}, E_{y}\right)$. Se $x=y$, nós temos que $L_{G}\left(E_{x}, E_{x}\right)$ é o espaço de $G$-automorfismos, denotado por $\operatorname{Aut}_{G}\left(E_{x}\right)=G L\left(E_{x}\right)$ e é um subgrupo de End $\left(E_{x}\right)$, isomorfo à $G$. Logo nós temos que a álgebra de Lie $\mathfrak{g}$ de $G$ é um subespaço de $\operatorname{End}\left(E_{x}\right)$ chamado de $\operatorname{End}_{\mathfrak{g}}\left(E_{x}\right)$.

Definition $9 \nabla \in \mathcal{C}(E)$ é admissível se para toda curva $\sigma:[0,1] \rightarrow M$, o transporte paralelo $P_{\sigma}$ for um G-mapa entre $E_{\sigma(0)}$ e $E_{\sigma(0)}$.

No exemplo anterior, do fibrado ortonormal sobre $M$, conexões compatíveis com a métrica, i.e.: tais que $\nabla\langle\cdot, \cdot\rangle=\langle\nabla(\cdot), \cdot\rangle+\langle\cdot, \nabla(\cdot)\rangle$ claramente levarão bases ortonormais em bases ortonomais por transporte paralelo.

Theorem $15 \nabla$ é admissível se e somente se, para todo $x \in M$ e todo $v \in T_{x} M$, em termos de bases admissíveis, $\omega(v) \in \mathfrak{g}$ no sentido que existe uma representação $d \rho: \mathfrak{g} \rightarrow E n d\left(E_{x}\right)$ tal que $\omega(v)=d \rho(h)$ para algum $h \in \mathfrak{g}$.

Dem: Se $\nabla$ é admissível, então para todo $v \in T_{x} M$ tal que $\sigma^{\prime}(0)=v, \sigma:[0,1] \rightarrow M$, $P_{\sigma}(0, t)$ é um $G$-mapa, ou seja, leva bases admissíveis em bases admissíveis. Seja $E$ um $G$-fibrado sobre $M$, e $\left\{e_{i}\right\}_{i=1}^{k}=e$ referencial sobre $\theta$. Designaremos uma base para $\sigma^{*}\left(E_{\mid \theta}\right)$ dada por $\left\{\left.e_{i}\right|_{(\sigma(t)}\right\}_{i=1}^{k}=\left\{\sigma^{*} e_{i}\right\}_{i=1}^{k}$. Simplesmente utilizando (1.98) para seções de campos do referencial, obtemos:

$$
\omega(v) e_{i}(\sigma(0))=\frac{d}{d t}{ }_{t=0}\left(P_{\sigma}(0, t) e_{i}(\sigma(t))\right)
$$

Mas $\left(\left(P_{\sigma}(0, t) e_{1}(\sigma(t)), \cdots,\left(P_{\sigma}(0, t) e_{k}(\sigma(t))\right)=P_{\sigma}(0, t) e(\sigma(t))\right.\right.$ é uma curva lisade referenciais admissíveis em $\sigma(0)$. Tendo escolhido $e(\sigma(0))$ como referencial admissível em $E_{\sigma(0)}$ há uma bijeção entre os referenciais admissíveis e $G$, portanto existe curva lisa $g:[0,1] \rightarrow G$ para a qual

$$
P_{\sigma}(0, t) e(\sigma(t))=\rho(g(t)) e(0)
$$

\footnotetext{
${ }^{9}$ Estamos identificando a representação de $g$, com $g$, i. e.: $\rho(g)=g$, ou seja, considerando a inclusão $G \subset \mathbf{G L}(k)$.
} 
Claramente $g(0)=I d$, portanto

$$
\frac{d}{d t}{ }_{\mid t=0} P_{\sigma}(0, t) e(\sigma(t))=d \rho\left(g^{\prime}(0)\right) e(0)
$$

e nós obtemos que $g^{\prime}(0) \in \mathfrak{g}$. Portanto

$$
\omega(v) e(0)=d \rho\left(g^{\prime}(0)\right) e(0)
$$

onde $e(0)$ é base em $\mathrm{x}$, logo, em relação a bases admissíveis, $\omega(v) \in \mathfrak{g}$ no sentido explicitado acima (omitiremos no restante dessa seção a representação para facilitar a notação).

Por outro lado suponhamos que $\omega(v) \in \mathfrak{g}$ para todo $v$. Seja $e(\sigma(t)$ curva de referenciais admissíveis sobre $\sigma$. Definimos $\tilde{e}(\sigma(t)):=P_{\sigma}(t, 0) e(\sigma(0))$. Nós temos que existe $h:[0,1] \rightarrow$ $G L(k)$ tal que para todo $t \in[0,1], e(\sigma(t))=h(t) \tilde{e}(\sigma(t))$. Claramente $h(0)=I d$. Agora

$$
\begin{gathered}
\omega\left(\sigma^{\prime}(t)\right) h(t) \tilde{e}(\sigma(t))=\omega\left(\sigma^{\prime}(t)\right) e(\sigma(t))=\nabla_{\sigma^{\prime}(t)} e(\sigma(t)) \\
=\nabla_{\sigma^{\prime}(t)} h(t) \tilde{e}(\sigma(t))=h^{\prime}(t) \tilde{e}(\sigma(t))+h(t) \nabla_{\sigma^{\prime}(t)} \tilde{e}(\sigma(t))=h^{\prime}(t) \tilde{e}(\sigma(t)) \\
\therefore \quad \omega\left(\sigma^{\prime}(t)\right) h(t)=h^{\prime}(t)
\end{gathered}
$$

Agora, usamos o seguinte lema provindo da teoria de grupos de Lie (para uma demonstração ver [16]):

Lemma 1 Dada uma curva lisa na álgebra de Lie $\xi:[0,1] \rightarrow \mathfrak{g}$, existe uma única curva lisa no grupo $G, g:[0,1] \rightarrow G$ tal que $g(0)=I d e$

$$
g^{-1}(t) g^{\prime}(t)=\xi(t)
$$

Nós sabemos que $G \subset G L(k), \mathfrak{g} \subset \mathfrak{g l}(k)$ e $h$ é então a única curva lisa em $G L(k)$ que satisfaz (1.108). Mas pelo lema existe uma única curva em $G$ que satisfaz $(1.108), \log h:[0,1] \rightarrow G$. E finalmente temos

$$
e(\sigma(t))=h(t) P_{\sigma}(t, 0) e(\sigma(0)) \Longrightarrow P_{\sigma}(t, 0) e(\sigma(0))=h^{-1}(t) e(\sigma(t))
$$

e portanto o transporte paralelo leva base admissível em base admissível.

Proposition 9 Se $\nabla$ é admissível, então $\Omega$ toma valores em $L_{\mathfrak{g}}(E, E) \subset L(E, E)$

Dem: Lembrando que podemos escrever a curvatura com $\Omega=d \omega+\omega \wedge \omega$ a demonstração torna-se trivial, já que $\mathfrak{g}$ é subespaço vetorial fechado por comutação.

\section{Gauges Quase Canônicos}

Seja $(\phi, \theta)$ um sistema de coordenadas convexo em $M$, centrado em $x_{0} \in \theta$, i.e.: tal que $\phi_{x_{0}}=0 \in \mathbb{R}^{n}$. Como em $\mathbb{R}^{n}$ existe uma escolha privilegiada de curva para ligar um dado ponto $\phi(y)$ e a origem, i.e.: $t \phi(y)$, podemos transportar esta estrutura para $M$ e com a ajuda do transporte paralelo escolher um referencial privilegiado relacionado à carta $\phi$. I.e.: dada uma base $\left\{v_{i}\right\}_{i=1}^{k}$ de $E_{x_{0}}$ a estendemos a um referencial local $\left\{e_{i}\right\}_{i=1}^{k}:=e$ tal que $e(y)=P_{\sigma}(t, 0) v$ onde definimos o "raio" ligando $x$ a $y$ como sendo $\sigma(t)=\phi^{-1}(t \phi(y))$ i.e.: 
$\phi(\sigma(t))=t \phi(p)$. Se $\nabla$ é uma conexão admissível e $v$ é uma base admissível em $x$, então $e(x)$ é uma base admissível em $\theta$ e chamada de gauge quase-canônico para $E$ sobre $\theta$.

Como já mencionamos, equações diferenciais ordinárias dependentes de parâmetros, têm dependência diferenciável não só nas condições iniciais mas também nos parâmetros. Logo $e(x)$ é um referencial liso. Além disso se $\omega$ é a forma de conexão relativa à $e(x), \omega\left(x_{0}\right) \equiv 0$.

Dem: Seja $u \in T_{x_{0}}$ e seja $\gamma:[-\epsilon, \epsilon] \rightarrow M$ tal que $\phi(\gamma(t))=t d \phi(u)$, então, como

$$
\frac{d}{d t}_{\mid t=0}(\phi(\gamma(t)))=d \phi\left(\gamma^{\prime}(0)\right)=d \phi(u) \therefore \gamma^{\prime}(0)=u
$$

Nós temos que a reparametrização $\tilde{\gamma}(t)=\gamma(\epsilon t):[0,1] \rightarrow M$ é o raio que liga $x$ a $\gamma(\epsilon)=y$. Ou seja,

$$
\phi(\gamma(\epsilon t))=\epsilon t d \phi(u)=t \phi(\gamma(\epsilon)) \Rightarrow \phi(\tilde{\gamma}(t))=t \phi(y)
$$

Agora

$$
\omega(u)\left(v_{i}\right)=\left.\frac{d}{d t}\right|_{t=0}\left(P_{\tilde{\gamma}}(0, t) e_{i}(\tilde{\gamma}(t))=\left.\frac{d}{d t}\right|_{t=0}\left(P_{\tilde{\gamma}}(0, t) P_{\tilde{\gamma}}(t, 0) v_{i}\right)=0\right.
$$

\section{A Derivada Covariante Exterior}

A derivada exterior que conhecemos até agora, heuristicamente falando, mesmo quando aplicada em elementos de $\Gamma\left(\Lambda^{p}\left(T M^{*}\right) \otimes E\right)$ só opera nas formas a valores reais, e só é válida para formas a valores em fibrados triviais (já que lá temos uma noção intrínseca de "deixar campos constantes"). Queremos uma derivada exterior que não seja assim limitada, que derive também a parte de seções, que leve em conta tanto as características usuais quanto a conexão em $E$. A essa derivada chamaremos de derivada exterior de gauge e denotaremos por $D_{p}^{\nabla}$. Dado que $\Gamma\left(\Lambda^{p}\left(T M^{*}\right) \otimes E\right)$ é gerado por elementos da forma $\lambda \otimes s$, onde $\lambda \in \Gamma\left(\Lambda^{p}(M)\right)$ e $s \in \Gamma(E)$, observando o critério acima, a forma natural de fazer isso seria:

$$
\widetilde{D}_{p}^{\nabla}(s \otimes \lambda)=s \otimes d \lambda+\nabla s \tilde{\wedge} \lambda
$$

Já que $\nabla s \in \Gamma\left(\Lambda\left(T M^{*}\right) \otimes E\right)$ e como vimos na seção 1.1.3, existe um produto exterior natural

$$
\Gamma\left(\Lambda^{p_{1}}\left(T M^{*}\right) \otimes E\right) \times \Gamma\left(\Lambda^{p_{2}}\left(T M^{*}\right)\right) \rightarrow \Gamma\left(\Lambda\left(T M^{*}\right)^{p_{1}+p_{2}} \otimes E\right)
$$

que chamaremos genericamente a partir agora também de $\wedge$ para quaisquer $p_{1}, p_{2}$, já que implicitamente fazemos a identificação do fibrado produto $\mathbb{R} \otimes E \simeq E$. Façamos um rápido interlúdio. Note o leitor que inadvertidamente invertemos a ordem $\lambda \otimes s \rightarrow s \otimes \lambda$. Porque o fizemos? Notemos que já para a derivada exterior da multiplicação de uma função $g: M \rightarrow \mathbb{R}$ por uma forma fechada $d \lambda$, nós temos que se usarmos a regra de Leibniz com uma ordem obtemos um resultado diferente do que com outra: $d(d f \otimes g)=d f \wedge_{\mathbb{R}} d g=-d g \wedge_{\mathbb{R}} d f=$ $-d(g \otimes d f)$. Mas nós definimos a derivada exterior do produto de uma função por uma forma como sendo $d(g \otimes d f)$. Como estamos perseguindo uma analogia entre a derivada exterior e a derivada exterior covariante, escolhemos aqui a mesma ordenação na definição. Como mostraremos essa condição será também necessária para a compatibilidade das duas em um 
sentido que veremos adiante. No entanto, como estamos usando um conceito de "formas a valores em fibrados vetoriais" e portanto estamos ordenando os elementos como $\lambda \otimes s$, definimos:

$$
\widetilde{D}_{p}^{\nabla}(\lambda \otimes s)=d \lambda \otimes s+(-1)^{p} \lambda \tilde{\wedge} \nabla s
$$

A forma de (1.112) sugere fortemente que a transição equivale a fazer uma substituição das derivadas normais de funções a valores reais, para derivadas covariantes de seções de fibrados.

Explicitando: como mencionamos anteriormente, localmente sempre podemos expandir $\eta \in \Gamma\left(\Lambda^{p}\left(T M^{*}\right) \otimes E\right)$ de maneira unívoca em termos de uma base $d x^{I} \otimes e_{i}$, onde $\left\{e_{i}\right\}_{i=1}^{k}$ é base local de $\Gamma(E),\left\{d x^{i}\right\}_{i=1}^{n}$ são coordenadas locais em $M$ e o superscrito maiúsculo $I$ é a notação de multi-índices (com ordem $p$ ).

Portanto localmente escrevemos $\eta=f_{I}^{i} d x^{I} \otimes e_{i}=d x^{I} \otimes f_{I}^{i} e_{i}$, então por (1.112) temos, para $f_{1}^{i}: \theta \subset M \rightarrow \mathbb{R}$

$$
\widetilde{D}_{p}^{\nabla} \eta=\widetilde{D}_{p}^{\nabla}\left(d x^{I} \otimes f_{I}^{i} e_{i}\right)=(-1)^{p} d x^{I} \wedge \nabla\left(f_{I}^{i} e_{i}\right)
$$

Ou seja, ao generalizar formas a valores reais para formas a valores em fibrados vetoriais, devemos também generalizar a equação $d\left(f_{I} \otimes d x^{I}\right)=d\left(f_{I} d x^{I}\right)=d f_{I} \wedge_{\mathbb{R}} d x^{I}=(-1)^{p} d x^{I} \wedge_{\mathbb{R}} d f_{I}$, relamente substituindo a derivada exterior usual pela derivada covariante!

Finalmente, dado $\nabla \in \mathcal{C}(E)$, devidamente motivados, simplesmente substituímos em (1.36) a derivação por $X$ pela derivação $\nabla_{X}$, ou seja, $d \rightarrow \nabla$. Definimos então os mapas lineares:

$$
D_{p}^{\nabla}=D_{p}: \Gamma\left(\Lambda^{p}(M) \otimes E\right) \rightarrow \Gamma\left(\Lambda^{p+1} \otimes E\right)
$$

que, para $X_{1}, \cdots, X_{p+1} \in \Gamma(T M)$, é dado por

$$
\begin{aligned}
\left(D_{p} \eta\right)\left(X_{1}, \cdots, X_{p+1}\right)= & \sum_{i=1}^{p+1}(-1)^{i+1} \nabla_{X_{i}} \eta\left(X_{1}, \cdots, \hat{X}_{i}, \cdots X_{p+1}\right)+ \\
& \sum_{i=1}^{p+1}(-1)^{i+j} \eta\left(\left[X_{i}, X_{j}\right], X_{1}, \cdots, \hat{X}_{i}, \cdots, \hat{X}_{j}, \cdots, X_{p+1}\right)
\end{aligned}
$$

Para mostrar que $D_{p} \eta \in \Gamma\left(\Lambda^{p+1} \otimes E\right)$, basta notar que por definição $D_{p} \eta$ é totalmente anti-simétrica e que é $C^{\infty}(M)$ linear em cada entrada, já que $X[f]=\nabla_{X} f$, e portanto a demonstração é exatamente a mesma que para (1.36). Com essa definição não estamos mais restritos a tomar elementos da forma $\lambda \otimes s$, o que é útil já que só podemos decompor um elemento de $\Gamma\left(\Lambda^{p}\left(T M^{*}\right) \otimes E\right)$ de forma única para cada base, i.e.: localmente.

Theorem 16 Se $\nabla^{i}$ é uma conexão em $E_{i}, i=1,2$ e $\nabla$ é a conexão correspondente em $E_{1} \otimes E_{2}$, então, para $\eta^{1} \in \Gamma\left(\Lambda^{p_{1}}\left(T M^{*}\right) \otimes E_{1}\right)$ e $\eta^{2} \in \Gamma\left(\Lambda^{p_{2}}\left(T M^{*}\right) \otimes E_{2}\right)$ temos

$$
D_{p_{1}+p_{2}}^{\nabla}\left(\eta^{1} \tilde{\wedge} \eta^{2}\right)=D_{p_{1}}^{\nabla_{1}} \eta^{1} \tilde{\wedge} \eta^{2}+(-1)^{p_{1}} \eta^{1} \tilde{\wedge} D_{p_{2}}^{\nabla_{2}} \eta^{2}
$$

Dem: Se escolhermos em $x$ um gauge quase canônico, então as formas de conexão relativas a $\nabla^{i}$ são nulas, i.e.: $\omega^{i}(x)=0, \operatorname{logo} \nabla^{i}=d$ e portanto $\nabla=d \otimes I d+I d \otimes d$ que é 
simplesmente a regra da cadeia. Portanto a equação (1.113) volta à forma de (1.36), para a qual já demonstramos que vale o teorema.

Gauges quase canônicos são instrumentos poderosos na teoria de fibrados. Para ilustrarmos o quanto eles podem facilitar nossa vida, bem como para demonstrarmos como bases locais nos podem ser úteis, daremos outra demonstração do teorema, mais trabalhosa, mas também elucidativa.

Expressemos $\eta^{1}$ e $\eta^{2}$ localmente por uma base como acima, i.e.: usando multi-índices $I$ e $J$ de ordem $p_{1}$ e $p_{2}$ respectivamente, $\left\{e_{i}\right\},\left\{b_{i}\right\}$ bases de $\Gamma\left(E_{1}\right)$ e $\Gamma\left(E_{2}\right)$, respectivamente, e $f_{I}^{i}, g_{J}^{j}: M \rightarrow \mathbb{R}$, temos: $\eta^{1}=f_{I}^{i} d x^{I} \otimes e_{i}, \eta^{2}=g_{J}^{j} d x^{J} \otimes b_{j}$. Utilizando (1.112) e $\nabla=\nabla^{1} \otimes I d+I d \otimes \nabla^{2}:$

$$
\begin{aligned}
\eta^{1} \tilde{\wedge} \eta^{2} & =\left(f_{I}^{i} e_{i} \otimes g_{J}^{j} b_{j}\right) \otimes\left(d x^{I} \wedge_{\mathbf{R}} d x^{J}\right) \\
D_{p_{1}+p_{2}}^{\nabla}\left(\eta^{1} \tilde{\wedge} \eta^{2}\right) & =\nabla\left(f_{I}^{i} e_{i} \otimes g_{J}^{j} b_{j}\right) \otimes\left(d x^{I} \wedge_{\mathbf{R}} d x^{J}\right) \\
& =\left(\nabla^{1}\left(f_{I}^{i} e_{i}\right) \otimes g_{J}^{j} b_{j}+f_{I}^{i} e_{i} \otimes \nabla^{2}\left(g_{J}^{j} b_{j}\right)\right)\left(d x^{I} \wedge_{\mathbf{R}} d x^{J}\right) \\
& =D_{p_{1}}^{\nabla^{1}}\left(f_{I}^{i} e_{i} \otimes d x^{I}\right) \tilde{\wedge}\left(g_{J}^{j} b_{j} \otimes d x^{J}\right)+(-1)^{p_{1}}\left(f_{I}^{i} e_{i} \otimes d x^{I}\right) \tilde{\wedge} D_{p_{2}}^{\nabla^{2}}\left(g_{J}^{j} b_{j} \otimes d x^{J}\right) \\
& =D_{p_{1}}^{\nabla^{1}} \eta^{1} \tilde{\wedge} \eta^{2}+(-1)^{p_{1}} \eta^{1} \tilde{\wedge} D_{p_{2}}^{\nabla^{2}} \eta^{2}
\end{aligned}
$$

A partir de agora abreviaremos $D_{p}^{\nabla}$ por $D$, deixando os índices subentendidos no contexto. Por esse resultado, tiramos imediatamente dois corolários úteis:

Proposition $10 \lambda^{1}$ for uma p-forma e $\lambda^{2}$ uma $p^{\prime}$-forma a valores em $E$, nós temos:

$$
D\left(\lambda^{1} \wedge \lambda^{2}\right)=d \lambda^{1} \wedge \lambda^{2}+(-1)^{p} \lambda^{1} \wedge D \lambda^{2}
$$

Proposition 11 Se $\lambda \in \Gamma\left(\Lambda^{p}\left(T M^{*}\right)\right)$ e $s \in \Gamma(E)$, então

$$
D_{p}^{\nabla}(\lambda \otimes s)=d \lambda \otimes s+(-1)^{p} \lambda \wedge \nabla s
$$

Ou seja, a operação de $D$ sobre elementos dessa forma é a apropriada ( $D$ estende $\widetilde{D}$ ).

De onde tiramos que, ao localmente escrevermos para $\eta \in \Gamma\left(\Lambda^{p}\left(T M_{\mid \theta}^{*}\right) \otimes E_{\mid \theta}\right)$ em relação a uma base $\left\{e_{i}\right\}$ de $\Gamma(E)$, i.e.: $\eta=\eta^{i} \otimes e_{i}$ para $\eta^{i} \in \Gamma\left(\Lambda^{p}\left(T M_{\mid \theta}^{*}\right)\right)$ obtemos:

$$
D \eta=D\left(\eta^{i} \otimes e_{i}\right)=d\left(\eta^{i}\right) \otimes e_{i}+(-1)^{p} \eta^{i} \wedge\left(\omega\left(e_{i}\right)\right)=d\left(\eta^{i}\right) \otimes e_{i}+\omega \wedge\left(\eta^{i} \otimes e_{i}\right)
$$

Logo

$$
D \eta=d \eta+\omega \wedge \eta
$$

Onde utilizamos a derivada exterior sobre formas em fibrados triviais, $d$ e em $\omega \wedge \eta$ o produto exterior que incorpora a ação bilinear $\operatorname{End}(E) \otimes E \rightarrow E$, resultante da ação da forma de conexão sobre as seções de $E$.

Nós sabemos que $d^{2}=d \circ d=0$ expressa justamente o fato de que as derivada ordinárias usuais comutam. Isto é, se $f: M \rightarrow \mathbb{R}, X, Y \in \Gamma(T M)$, calculamos

$$
d(d f)(X, Y)=X[d f(Y)]-Y[d f(X)]-d f([X, Y])=(X Y-Y X-[X, Y]) f=0
$$


Novamente, fazendo a substituição $d \rightarrow D$, i.e.: $X \rightarrow \nabla_{X}$ em (1.116) obtemos

$$
\nabla_{X} \nabla_{Y}-\nabla_{Y} \nabla_{X}-\nabla_{[X, Y]}=\Omega(X, Y)
$$

Logo devemos ter $D_{1} \circ D_{0}=i_{\Omega}$ (produto interior por $\Omega$ ). De fato, se $\eta$ é uma p-forma a valores em $E$, utilizando (1.115) temos:

$$
\begin{aligned}
D(\eta) & =d \eta+\omega \wedge \eta \\
D(D(\eta)) & =d \omega \wedge \eta-\omega \wedge d \eta+\omega \wedge d \eta+\omega \wedge \omega \wedge \eta \\
& =\Omega \wedge \eta
\end{aligned}
$$

Theorem 17 Dada conexão $\nabla$ em $E$, seja $\widetilde{\nabla}$ a conexão induzida em $\operatorname{End}(E) \simeq E^{*} \otimes E$. Se $\omega$ é a matriz de 1-formas de conexão para $\nabla$ relativa à base $\left\{e_{i}\right\}$ e $\alpha$ é uma p-forma em $M$ a valores em $\operatorname{End}(E)$, utilizando o produto externo natural entre formas a valores em End(E) que descrevemos na seção 1.1.3 temos:

$$
D^{\tilde{\nabla}} \alpha=d \alpha+\omega \wedge \alpha-(-1)^{p} \alpha \wedge \omega
$$

Dem: Novamente, utilizando (1.115) para formas a valores no fibrado vetorial $\operatorname{End}(E)$, temos:

$$
D^{\tilde{\nabla}} \alpha=d \alpha+\widetilde{\omega} \wedge \alpha
$$

onde assim como em (1.115), temos de deixar a ação da forma sobre as seções do fibrado implícitas no produto exterior, o que aqui, ao invés de induzir a identificação $\operatorname{End}(E) \otimes E \rightarrow$ $E$, induz $L(\operatorname{End}(E), \operatorname{End}(E)) \otimes \operatorname{End}(E) \rightarrow \operatorname{End}(E)$.

Se $\widetilde{\omega}$ é a conexão de $\widetilde{\nabla}=\nabla \otimes \mathrm{Id}+\mathrm{Id} \otimes \nabla^{*}$, lembremos que $\widetilde{\omega}=\omega \otimes \mathrm{Id}+\mathrm{Id} \otimes \omega^{*}$. Mas como vimos, na identificação $\operatorname{End}(E) \simeq E \otimes E^{*}$, temos Id $\otimes \omega^{*}=-\operatorname{Id} \otimes \omega^{T}$. Utilizaremos ainda que $\omega^{T}\left(e^{j}\right)=e^{j} \omega$, fato já comentado. Portanto escrevendo localmente $\alpha=\alpha_{j}^{i} \otimes e_{i} \otimes e^{j}$ obtemos:

$$
\begin{aligned}
D \alpha & =D\left(\alpha_{j}^{i} \otimes e_{i} \otimes e^{j}\right) \\
& =d\left(\alpha_{j}^{i}\right) \otimes\left(e_{i} \otimes e_{j}\right)+(-1)^{p} \alpha_{j}^{i} \wedge\left(\omega\left(e_{i}\right) \otimes e^{j}-e_{i} \otimes \omega^{T}\left(e^{j}\right)\right) \\
& =d\left(\alpha_{j}^{i}\right) \otimes e_{i} \otimes e^{j}+\omega \wedge\left(\alpha_{j}^{i} \otimes e_{i} \otimes e^{j}\right)-(-1)^{p} \alpha_{j}^{i} \wedge e_{i} \otimes e^{j} \omega \\
& =d \alpha+\omega \wedge \alpha-(-1)^{p} \alpha \wedge \omega
\end{aligned}
$$

Já que a multiplicação exterior de matrizes de formas é simplesmente a multiplicação de matrizes usando o produto externo em cada termo.

Proposition 12 Deste teorema emergem os seguintes corolários:

(i) A identidade de Bianchi (1.72) é equivalente à $D \Omega=0$.

(ii) $D \omega=\Omega$ 
Dem: Nós temos $d \Omega=\omega \wedge \Omega-\Omega \wedge \omega$, portanto tomando no último teorema $\alpha=\Omega$ obtemos

$$
D \Omega=d \omega+\omega \wedge \Omega-\Omega \wedge \omega
$$

O segundo item se verifica similarmente tomando $\alpha=\omega$ em (1.115).

Agora, dada conexão $\nabla$ em $E$ e $\kappa \in \Gamma\left(\Lambda^{1}\left(T M^{*}\right) \otimes \operatorname{End}(E)\right)$, definimos $\nabla^{\kappa}=\nabla+\kappa$. Se tivermos uma base local isto é equivalente a tomar, para a forma de conexão de $\nabla^{\kappa}$, $\omega^{\kappa}=\omega+\kappa$ então obtemos:

$$
\begin{gathered}
d(\omega+\kappa)+(\omega+\kappa) \wedge(\omega+\kappa)=d \omega+d \kappa+\omega \wedge \omega+\kappa \wedge \omega+\omega \wedge \kappa+\kappa \wedge \kappa= \\
\Omega+\left(d \kappa+\omega \wedge \kappa-(-1)^{1} \kappa \wedge \omega\right)+\kappa \wedge \kappa
\end{gathered}
$$

Logo obtemos a partir de (1.117):

$$
\Omega^{\kappa}=\Omega+D \kappa+\kappa \wedge \kappa
$$

Nós voltaremos à identidade de Bianchi quando discutirmos as equações de Yang-Mills. Nós veremos por exemplo que em eletromagnetismo ela representa consevação de carga, e é verdade que em relatividade geral, pela equação de Einstein ela é equivalente à conservação local de energia e momento. 


\section{Chapter 2}

\section{Fibrados Principais}

O Universo é embasado em um plano, um plano cuja profunda simetria está de alguma forma presente na estrutura interna do nosso intelecto. - Paul Valery

Einstein em seu tempo trabalhou incessantemente para construir "um sistema completo de física teórica". Ele procurou os "conceitos e princípios fundamentais" que permitiriam uma grande síntese da estrutura do mundo real. Centrais a essa síntese estão as forças, ou interações que mantêm unida a matéria, que produzem a pletora de reações de que consistem os fenômenos naturais. Eu acredito que ainda estamos hoje muito longe desta grand síntese com a qual sonhou Einstein. Mas nós temos um de seus elementos chave: o princípio que interações são regidas por simetrias, utilizado primeiramente pelo próprio Einstein. - C.N. Yang

\subsection{Fundamentos}

Em muitos sentidos, a Teoria de Gauge é uma teoria que procura a natureza intrínseca das interações, aquilo que independe das formas como elas são representadas. Como um objeto ao meio de uma roda de observadores, cuja descrição é feita a partir de diferentes ângulos (" cada uma delas correta"), a Teoria de Gauge tenta desemaranhar propriedades inerentes de propriedades descritivas. Assim como para o objeto, as descrições são relacionadas por transformações de simetria, por um grupo de simetria. Estudaremos agora uma forma de estudarmos as relações entre as descrições feitas por estes diferentes observadores, ou referenciais.

Ao introduzirmos bases admissíveis na seção 1.4, chamamos $\left\{e_{i}\right\}_{i=1}^{k}$ e $\left\{\tilde{e}_{i}\right\}_{i=1}^{k}$ de bases admissíveis de $E_{x}$ se existisse $g \in G$ tal que $\left\{e_{i}\right\}_{i=1}^{k}=\left\{g \tilde{e}_{i}\right\}_{i=1}^{k}$. Este $g$ é único já que existe um único $g \in G L(k) \supset G$ que leva uma na outra, então concluímos que uma vez escolhida uma base inicial (uma origem), existe uma bijeção entre as bases admissíveis e o grupo $G$. Notemos ainda que não há uma base que se destaca das outras, não há uma base que possa ser considerada canonicamente como a identidade. Como veremos ao final deste capítulo, o fibrado dos referenciais admissíveis de $E$, constitui uma ponte entre as noções de fibrado vetorial e principal.

Motivados por essas construções, definimos: 
Definition 10 Uma variedade diferenciável $P$ é chamada de fibrado principal com grupo associado $G$, se $G$ age livre e diferenciávelmente sobre $P$. Ou seja, se existe ação de $G$ em $P: G \times P \rightarrow P$ lisa e tal que para cada $p \in P$ o grupo de isotropia de $p$ é a identidade, i.e.:

$$
G_{p}=\{y \in G \mid g p=p\}=\{e\}
$$

Naturalmente construímos uma projeção em $P, \pi: P \rightarrow M$, dada por $p \simeq q \Leftrightarrow p=g \cdot q$ para algum $g \in G$. Definimos então o espaço base $M$ como sendo o espaço das órbitas de $P, M=P / G$, com a topologia quociente, i.e.: caracterizada por $\pi$ ser aberta e contínua. Pela definição, $G$ age transitivamente sobre cada fibra.

\section{Ações de Grupos de Lie}

Antes de começar o tratamento de fibrados principais em si, precisamos de algumas ferramentas da teoria de grupos de Lie: Seja, $G$ um grupo Lie compacto e $\mathfrak{g}$ sua álgebra de Lie, ou seja, $\mathfrak{g} \simeq T_{e} G$.

Definimos a ação de conjugação do grupo como sendo:

$$
\begin{array}{r}
\zeta(g): G \rightarrow G \\
a \mapsto g a g^{-1}
\end{array}
$$

Cuja derivada em $e \in G$ denotaremos por $\operatorname{Ad}(g)=d(\zeta(g))_{e}$. É fácil ver que $\zeta(g) \zeta(h)=\zeta(g h)$, então pela regra da cadeia, $\operatorname{Ad}(g h)=d(\zeta(g h))_{e}=d(\zeta(g))_{e} d(\zeta(h))_{e}=\operatorname{Ad}(g) \operatorname{Ad}(h)$, logo Ad : $G \rightarrow \operatorname{Aut}(\mathfrak{g})$ é uma representação do grupo sobre a álgebra. Definimos exptX como sendo o único subgrupo a 1-parâmetro tangente a $X$ em $e$.

Proposition $13 \zeta(g)(\exp t X)=\exp (t A d(g) X)$

Dem: Nós temos que $\zeta(g) e=e$, e $\zeta(g)(a b)=\zeta(g) a \zeta(g) b$, isto é: $\quad \zeta(g)$ é automorfismo de $G$, logo leva subgrupo a 1-parâmetro em subgrupo à 1-parâmetro. Logo $\zeta(g) \exp t X$ é subgrupo a 1-parâmetro que passa pela origem, com tangente

$$
\left.\frac{d}{d t}\right|_{t=o} \zeta(g) \exp t X=\operatorname{Ad}(g) X
$$

Por outro lado, $\exp (t \operatorname{Ad}(g) X)$ é o único subgrupo a 1-parâmetro que passa pela origem com tangente $\operatorname{Ad}(g) X$.

Seja $P$ uma variedade onde $G$ age como grupo de difeomorfismos (ação que denotaremos por '‘'. Então, para todo $p \in P, X \in \mathfrak{g}$ e $t \in \mathbb{R}$, nós temos uma ação

$$
\begin{aligned}
\exp (t X): P & \longrightarrow P \\
p & \longmapsto \exp (t X) \cdot p
\end{aligned}
$$

Logo definimos

$$
\begin{aligned}
\mathrm{I}_{p}: \mathfrak{g} & \longrightarrow T_{p} P \\
X & \left.\longmapsto \frac{d}{d t}\right|_{t=o}(\exp (t X) \cdot p)
\end{aligned}
$$


Portanto,

$$
\begin{aligned}
\mathrm{I}(X): P & \rightarrow \Gamma(T P) \\
p & \left.\mapsto \frac{d}{d t}\right|_{t=o}(\exp (t X) \cdot p)
\end{aligned}
$$

É claro que $\exp (t X) \cdot p=p \Leftrightarrow \mathrm{I}_{p}(X)=0 \mathrm{e}$, se a ação do grupo for livre, $\mathrm{I}_{p}$ é injetora. Além disso nós temos que:

$$
\operatorname{ImI}_{p}=\left\{\tilde{X} \in T_{p} P\left|\tilde{X}=\frac{d}{d t}\right|_{t=o}(\exp (t X) \cdot p) \text { para algum } X \in \mathfrak{g}\right\}
$$

Por outro lado, exp é difeomorfismo local e, portanto, $\exp (t X) \cdot p$ gera $G \cdot p$ em uma vizinhança ao redor de $p$. Logo, $T_{p} G \cdot p=\operatorname{Im} \mathrm{I}_{p}$. Denotando a aplicação ' $’: G \times P \rightarrow P$ por $\mu$, fica fácil ver que $\mathrm{I}_{p}$ é linear:

$$
\mathrm{I}_{p}(X)=\left.\frac{d}{d t}\right|_{t=o}(\Phi(\exp (t X), p))=d \mu_{p}(X, 0)
$$

Além disso nós temos, pela última proposição que

$$
\begin{array}{r}
\mathrm{I}_{p}\left(\operatorname{Ad}\left(g^{-1}\right) X\right)=\left.\frac{d}{d t}\right|_{t=o}\left(\exp t\left(\operatorname{Ad}\left(g^{-1}\right) X\right) \cdot p\right)=\left.\frac{d}{d t}\right|_{t=o}\left(g^{-1} \exp (t X) g \cdot p\right) \\
\therefore d g\left(\mathrm{I}_{p}\left(\operatorname{Ad}\left(g^{-1}\right) X\right)\right)=\left.\frac{d}{d t}\right|_{t=o}(\exp (t X) g \cdot p)=\mathrm{I}_{g \cdot p}(X)
\end{array}
$$

Obtemos então as duas propriedades

- $\operatorname{Im}\left(\mathrm{I}_{p}\right)=$ espaço tangente à órbita $G \cdot p$ em $p$.

- A ação de I em um ponto $q$ transladado do ponto $p$ por um elemento $g$ de $G$ é relacionada àquela no ponto $p$ da seguinte forma:

$$
\mathrm{I}_{g \cdot p}(X)=d g\left(\mathrm{I}_{p}\left(\operatorname{Ad}\left(g^{-1}\right) X\right)\right)
$$

\section{Métricas $G$-invariantes}

$\mathrm{Na}$ teoria de fibrados principais, atenção especial deve ser dada à estruturas $G$-ivariantes, isto é, às estruturas em $P$ que não se alteram sob a ação do grupo. Este em si é um tópico rico e profundo, que não abordaremos em sua generalidade, mas somonte no tocante à uma estrutura: a métrica. Para uma abordagem mais profunda veja [?].

Definition 11 Seja $\gamma$ uma métrica sobre $P$, dizemos que $\gamma$ é $G$-invariante se $G$ está contido no grupo de isometrias de $\gamma$. I.e.: se para todo $g \in G, g^{*} \gamma=\gamma$.

Uma questão que naturalmente surge é sobre a generalidade da existência de tais métricas. Mostraremos que sempre existem para o caso de $G$ compacto e conexo.

Definition 12 A média de $\gamma$ por $G$ (compacto, conexo e de dimensão= $m$ ) é dada ponto a ponto por:

$$
\tilde{\gamma}=\int_{g \in G} g^{*}(\gamma) \nu
$$

onde $\nu$ é uma m-forma volume bi-invariante. (que sempre existe se $G$ é compacto e conexo). 
Theorem 18 Seja $\gamma$ uma métrica em uma variedade riemanniana $P$ onde $G$ age como grupo de difeomorfismos, então a média de $\gamma$ é $G$-invariante.

Dem: Sejam $u, v \in T_{p} P, h \in G$, definimos $f: G \rightarrow \mathbb{R}$ por $f(g)=g^{*} \gamma(u, v)$, então

$$
\begin{array}{r}
\tilde{\gamma}(u, v)=\int_{g \in G} \gamma\left(g_{*}(u), g_{*}(v)\right) \nu=\int_{g \in G} f(g) \nu \quad \therefore \\
h_{*} \tilde{\gamma}(u, v)=\tilde{\gamma}\left(h_{*} u, h_{*} v\right)=\int_{g \in G} \gamma\left(g_{*}\left(h_{*}(u)\right), g_{*}\left(h_{*}(v)\right)\right) \nu= \\
\left.\int_{g \in G} \gamma\left(g_{*} h_{*}(u), g_{*} h_{*}(v)\right)\right) \mathrm{R}_{h}^{*}(\nu)=\int_{g \in G}\left(\mathrm{R}_{h}^{*} f\right)(g) \mathrm{R}_{h}^{*}(\nu)
\end{array}
$$

Mas a translação à direita $\mathrm{R}_{h}: G \rightarrow G$ é um difeomorfismo que preserva a orientação, logo, $h_{*} \tilde{\gamma}=\tilde{\gamma}$. Dado que toda variedade diferenciável admite métrica riemanniana, o teorema está provado.

\section{Seções no Fibrado Principal}

Tentaremos agora, assim como assumimos em fibrados vetoriais, demonstrar que existe uma estrutura local de variedade produto também para os fibrados principais, onde a fibra típica coincide com o grupo associado, $G$.

Definition 13 Seja $\theta$ um aberto em $M$, definimos uma seção local de $P$ sobre $\theta$ como uma subvariedade $\Sigma$ de $P$ tal que $\Sigma$ é transversal às órbitas, $T_{p} \Sigma+I_{p}(\mathfrak{g})=T_{p} P$, e $\Sigma$ intersecta órbitas em um único ponto, i.e.: se $p \in \Sigma$ então $G \cdot p \cap \Sigma=\{p\}$.

Theorem 19 Dado $p \in P$, existe uma seção local $\Sigma$ de $P$ contendo $p$.

Dem: A idéia da prova é, usando uma métrica invariante em $P$, exponenciar os vetores ortogonais a $G \cdot p=: N$, de comprimento $\delta$, obtendo uma outra subvariedade, $\Sigma$, transversal a $N$. Devemos tomar o cuidado necessário para que $\delta$ seja suficientemente pequeno de modo que haja uma só interseç̧ão entre $\Sigma$ e $N$. Provaremos um caso mais geral e depois mostraremos que nosso caso se encaixa. Provamos antes de mais nada que $G \cdot p$ é subvariedade mergulhada:

Para todo $p \in P$, definimos o mapa suave $\theta^{(p)}: G \rightarrow P$ por $\theta^{(p)}(g)=g \cdot p$, ou seja a órbita de $p$. Como o grupo age livremente é trivial ver que $\theta^{(p)}$ é injetora. Além disso como existe identificação canônica $d \theta^{(p)} \simeq \mathrm{I}_{p}$, por (2.1) o posto de $\theta^{(p)}$ é constante sobre $G$. Agora pelo teorema do posto, existem cartas apropriadas de $G$ e $P$ tal que a representação local de $\theta^{(p)}$, que chamamos de $\widetilde{\theta}^{(p)}$, pode ser escrita como $\widetilde{\theta}^{(p)}\left(x^{1}, \ldots, x^{n}\right)=\left(x^{1}, \ldots, x^{j}, 0, \ldots, 0\right)$ onde $n=\operatorname{dim} G$. Mas como é injetora, $j=n$, i.e.: é uma imersão. Agora, como $G$ é compacto, nós temos uma imersão injetora de um compacto, que é portanto mergulho.

Seja então $N$ subvariedade compacta de $P$, definimos o fibrado normal de N:

$$
\nu N=\left\{(x, v) \mid x \in N, v \in \nu_{x} N\right\}
$$

É claro que $\nu N$ é fibrado vetorial, já que $T N$ é distribuição $C^{\infty}$ em $T P$ e portanto o seu ortogonal também é uma distribuição lisa, o que transforma $\nu N$ em sub-fibrado de $T P$. Achemos então uma trivialização local de $\nu N$. 
Tomamos primeiramente uma carta de $P$ adaptada à $N, \psi: U \rightarrow \widetilde{U} \subset \mathbb{R}^{m}$. Agora, pela propriedade de carta adaptada à subvariedade, temos que a projeção nas $n$ primeiras coordenadas, $p r^{n} \circ \psi: N \cap U \rightarrow \mathbb{R}^{n} \cap \widetilde{U}$ é carta de $N \cap U$. Seja $k=m-n$, e $p r^{k}$ a projeção nas últimas $k$ coordenadas. Claramente $f:=p r^{k} \circ \psi$ é submersão e $U \cap N=f^{-1}(0)$, além disso $\operatorname{Ker} d f_{p}=T_{p} N$. Agora ${ }^{1}$, induzindo uma métrica em $R^{m}$ por $\psi$ (e portanto em $\mathbb{R}^{k}$ por $f)$, denotamos o adjunto de $d f_{p}$ por $d f_{p}^{*}$, que é definido por dados $u \in T_{p} P$ e $w \in \mathbb{R}^{k}=\operatorname{Im} d f_{p}$ :

$$
\left\langle d f_{p}(u), w\right\rangle_{\mathbf{R}^{k}}=\left\langle u, d f_{p}^{*}(w)\right\rangle_{T_{p} P}
$$

Claramente, como $d f_{p}$ é isometria, $d f_{p}^{*}=d f_{p}^{-1}$. Agora se $u \in T_{p} N$, qualquer que seja $w \in \mathbb{R}^{k},\left\langle u, d f_{p}^{-1}(w)\right\rangle_{T_{p} P}=0$. Isto é, $\operatorname{Im} d f_{p}^{-1} \subset T_{p} N^{\perp}$, mas ambos têm dimensão $k$, logo $d f_{p}^{-1}: \mathbb{R}^{k} \rightarrow \nu_{p} N$ é isomorfismo. Logo construímos uma trivialização local para $\nu N$ dada por

$$
\begin{gathered}
\phi: \nu N_{\mid N \cap U} \longrightarrow(U \cap N) \times \mathbb{R}^{k} \\
(p, u) \longmapsto\left(p, d f_{p}(u)\right)
\end{gathered}
$$

Utilizando a construção de fibrado tangente (que vimos nos exemplos da Seção 1) é fácil vermos que trivializações dadas por cartas adaptadas $\psi, \widetilde{\psi}$, compatíveis, serão também compatíveis. Chamaremos a projeção suave deste fibrado vetorial de $\pi_{N}: \nu N \longrightarrow N$. Estudemos então a aplicação $\operatorname{Exp}: \nu N \rightarrow P$, que é simplesmente a restrição da aplicação exponencial usual em $T P$ à distribuição normal a $N$. Todo cuidado é pouco ao estudarmos fibrados tangentes de fibrados vetoriais, por isso para facilitar a vizualização utilizamos que $(U \cap N) \times \mathbb{R}^{k}$ é fibrado trivial, e, para $p \in N \cap U$, qualquer vetor $\xi \in T_{(p, 0)} \nu N$ é dado por $d \phi_{(p, 0)}^{-1} \tilde{\xi}$ para algum

$$
\tilde{\xi}=(w, u) \in T_{(p, 0)}\left((U \cap N) \times \mathbb{R}^{k}\right)=T_{p}(U \cap N) \times T_{0} \mathbb{R}^{k}
$$

que por sua vez é tangente a uma curva $(\gamma(t), t u)$, onde $\gamma:[0,1] \rightarrow N \cap U$. Ou seja, fazendo $(p, 0)=q$ :

$$
\begin{gathered}
\xi=d \phi_{q}^{-1}(w, u)=d \phi_{q}^{-1}(w, 0)+d \phi_{q}^{-1}(0, u) \\
=d \phi_{q}^{-1}\left(\left.\frac{d}{d t}\right|_{t=0}((\gamma(t), 0))\right)+d \phi_{q}^{-1}\left(\left.\frac{d}{d t}\right|_{t=0}((p, t u))\right)=\left.\frac{d}{d t}\right|_{t=0}\left(\phi^{-1}(\gamma(t), 0)\right)+\left.\frac{d}{d t}\right|_{t=0}\left(\phi^{-1}(p, t u)\right) \\
=\left.\frac{d}{d t}\right|_{t=0}((\gamma(t), 0))+\left.\frac{d}{d t}\right|_{t=0}\left(\left(p, t d f_{p}^{-1}(u)\right)\right)=(w, 0)+\left(0, d f_{p}^{-1}(u)\right)
\end{gathered}
$$

\footnotetext{
${ }^{1}$ A forma mais óbvia de exibirmos uma subvariedade transversal a $N$ em $p$ seria simplesmente tomando a subvariedade dada por $\Sigma=\psi^{-1}\left(\mathbb{R}^{k} \cap \widetilde{U}\right)$, ou seja, os pontos $\psi^{-1}\left(0, \ldots, 0, x^{n+1}, \ldots, x^{m}\right)$ onde $\left(0, \ldots, 0, x^{n+1}, \ldots, x^{m}\right) \in \widetilde{U}$. No entanto, a propagação desta construção ao longo da fibra de forma $G-$ invariante se torna mais complicada do que o que faremos aqui utilizando a existência de uma métrica $G$-invariante.
} 
Obtemos uma identificação canônica $T_{(p, 0)} \nu N \simeq T_{p} N \oplus \nu_{p} N$. Finalmente, fazendo ${ }^{2}$, para $v \in T_{x} P, \operatorname{Exp}(x, v)=\operatorname{Exp}_{x}(v)$ temos:

$$
\begin{gathered}
(d \operatorname{Exp})_{q}(\xi)=\left.\frac{d}{d t}\right|_{t=o}(\operatorname{Exp}(\gamma(t), 0))+\left.\frac{d}{d t}\right|_{t=o}\left(\operatorname{Exp}\left(p, t d f_{p}^{-1}(u)\right)\right) \\
=\left.\frac{d}{d t}\right|_{t=o}((\gamma(t), 0))+\left.\frac{d}{d t}\right|_{t=o}\left(\operatorname{Exp}_{p}\left(t d f_{p}^{-1}(u)\right)\right)=(w, 0)+\left(0, d f_{p}^{-1}(u)\right)=\xi \\
\therefore d(\operatorname{Exp})_{q}=I d_{\mid T_{q} \nu N}
\end{gathered}
$$

que é válido para todos os pontos da forma $(p, 0)$. Logo, pelo teorema da função inversa, ao redor de cada ponto $p \in N$, existe um aberto $V \subset N$ e vizinhança da seção nula de $\nu N_{\mid \pi_{N}^{-1} V}$ que é levada difeomorficamente sobre a imagem. Lembremo-nos também que todo vetor de $T_{p} P$ pode ser escrito de forma única como soma de um vetor tangente à $N$ e um normal à $N$. Logo a aplicação exponencial leva um aberto de $\nu N_{\mid \pi_{N}^{-1} V}$ vizinhança da seção nula difeomorficamente sobre um aberto de $P$ que contém $V$. Podemos tomar essa vizinhança da seção nula de $\nu N_{\mid \pi_{N}^{-1} V}$ como sendo da forma $\phi^{-1}\left(V \times B_{\delta}(0)\right)$ para algum $\delta>0$.

Pictoricamente, estamos levando uma vizinhança tubular "reta" (já que tem seu diâmetro em um fibrado vetorial), para uma vizinhaça tubular "curva" ao redor de $N$ em $P$. Nós temos que para a própria seção nula, $\operatorname{Exp}: N \times\{0\} \rightarrow N$ é a identidade em $N$. É claro então que para cada ponto $p \in N$ a exponencial de $B_{\delta}\left(0_{p}\right):=B_{\delta}(0) \cap \nu_{p} N \subset \nu_{p} N$ é uma subvariedade de $P$ de dimensão $k$, transversal à $N$, que chamaremos de $\Sigma_{p}$.

Nós temos que a exponencial é então um difeomorfismo local. Contudo, pode ainda ocorrer que a exponencial não leva vizinhança global da seção nula de $\nu N$ injetoramente sobre a imagem, ou seja, que para qualquer raio global do tubo que tomarmos teremos auto-intersecção ao mandarmos o tubo para $P$ através da exponencial. Para completar a demonstração do teorema, temos de provar que existe vizinhança de $N$ onde a Exp é de fato injetora.

Proposition 14 Sejam $X, Y$ variedades suaves, $f: X \rightarrow Y C^{\infty}, f_{\mid N}$ injetora para uma dada $N$ subvariedade compacta de $X$ e $d f_{x}: T_{x} X \rightarrow T_{y} Y$ isomorfismo para $x \in N$. Então existe vizinhança aberta $U$ de $N$ em $X$ tal que $f: U \rightarrow f(U)$ é difeomorfismo.

Dem: Para provar o lema só nos resta provar injetividade. Seja então o conjunto das vizinhanças abertas de $N$ :

$$
\mathcal{C}=\{U \mid U \text { é vizinhança aberta de } N\}
$$

Construímos a ordem parcial em $\mathcal{C}$ pela inclusão inversa, i.e.: $W \geq U$ se $W \subset U$, então é fácil verificar que $\mathcal{C}$ é um conjunto dirigido. Seja $S$ um elemento de $\mathcal{C}$, e $x_{S}$ um ponto em $S$, então $\left\{x_{S}\right\}$ é uma rede em $X$. Para qualquer $W \in \mathcal{C}$, se $S \geq W, x_{S} \in W$. Logo como $N$ é compacto ( e portanto fechado) nós temos um lema de topologia geral que garante que uma rede $\left\{x_{S}\right\}$ converge para um ponto de $N$ (ver [18] ). Além disso, como $X$ é Hausdorff, este ponto é único. Notamos ainda que para qualquer vizinhança aberta $V$ de $x$, existe $W \in \mathcal{C}$, tal que para todo $S \geq W$ nós temos $x_{S} \in V$.

\footnotetext{
${ }^{2}$ Chamamos aqui a aplicação exponencial riemanniana de Exp, para diferenciá-la da exponencial no grupo, que chamaremos de exp.
} 
Uma função $f: X \longrightarrow Y$ é contínua se e somente se, para toda rede $\left\{x_{S}\right\}$,

$$
f\left(\lim _{S \in \mathcal{C}} x_{S}\right)=\lim _{S \in \mathcal{C}} f\left(x_{S}\right)
$$

Claramente, $f$ é injetora em alguma vizinhança aberta de $N$ se e somente se $f_{\mid S}$ é injetora para algum $S \in \mathcal{C}$. Logo, suponhamos por absurdo que para todo $S \in \mathcal{C}, f_{\mid S}$ não é injetora. Então qualquer que seja $S \in \mathcal{C}$, existem $x_{S} \neq y_{S} \in S$ tal que $f\left(x_{S}\right)=f\left(y_{S}\right)$. Montamos duas dessas redes: $\left\{x_{S}\right\}$ e $\left\{y_{S}\right\}$, que já sabemos convergir para pontos em $N, x$ e $y$ respectivamente. Como $f$ é contínua,

$$
\lim _{S \in \mathcal{C}} f\left(y_{S}\right)=\lim _{S \in \mathcal{C}} f\left(x_{S}\right)=f(x)=f(y)
$$

Mas $f$ é difeomorfismo de $N$ sobre sua imagem, em particular é injetora sobre $N$, então essa relação implica $x=y$. No entanto $f$ é difeomorfismno local ao redor de cada ponto de $N$, logo existe $V$ aberto $P$ tal que $x \in V$ onde $f$ é injetora. Portanto, como $y_{S}, x_{S} \longrightarrow x$, existe $W \in \mathcal{C}$ tal que para $S \geq W, x_{S} \neq y_{S}$ implica $f\left(x_{S}\right) \neq f\left(y_{S}\right)$ o que contraria a nossa hipótese. Ou seja, existe $S \in \mathcal{C}$ tal que se $x \neq y \in S, f(x) \neq f(y)$.

Dado esse resultado, como $N$ é compacta nós temos que existe um raio mínimo $\delta_{0}$ para o qual podemos achar um difeomorfismo entre o tubo " reto" dentro de $\nu N$ e o tubo "curvo" em $P$ obtido pela exponencial.

Voltando ao nosso caso, $\Sigma_{p}=\operatorname{Exp}_{p} B_{\delta}\left(0_{p}\right)$, e as órbitas que passam por $x \in \Sigma_{p}$ terão a forma $g \cdot \operatorname{Exp}_{p}(w)$ para algum $w \in \nu_{p} N$ e algum $g \in G$. Lembremos que colocamos em $P$ uma métrica $G$-invariante, portanto, como $g$ age isometricamente, leva geodésica em geodésica. Mas se $g \neq e$ então $g \cdot p \neq p$ e $g \cdot \operatorname{Exp}(p, t u)$ é uma geodésica que passa por $g \cdot p$ com tangente

$$
\left.\frac{d}{d t}\right|_{t=o}(g \cdot \operatorname{Exp}(p, t u))=\left.d g \circ \frac{d}{d t}\right|_{t=o}(\operatorname{Exp}(p, t u))=d g(u)
$$

assim como $\operatorname{Exp}(g \cdot p, t d g(u))$, então por unicidade,

$$
g \cdot \operatorname{Exp}(p, w)=\operatorname{Exp}\left(g \cdot p, d g_{p}(w)\right)
$$

Além disso, nós temos que, como $G$ age por isometrias, logo preserva a perpendicularidade do subespaço normal e o raio $\delta$, i.e.: $d g\left(B_{\delta}\left(0_{p}\right)\right)=B_{\delta}\left(0_{g \cdot p}\right) \subset \nu_{g \cdot p} N$ então $d g(u) \in \nu_{g \cdot p} N$. Mas como mostramos, para $\delta_{0}$ nós temos que:

$$
\operatorname{Exp}\left(g \cdot p, B_{\delta_{0}}\left(0_{g \cdot p}\right)\right) \cap \operatorname{Exp}\left(p, B_{\delta_{0}}\left(0_{p}\right)\right)=\emptyset
$$

e portanto teremos apenas uma intersecção entre cada órbita e $\Sigma_{p}$.

Finalmente, nós sabemos que $T_{p} \Sigma_{p}$ é transversal a $\mathrm{I}_{p}(\mathfrak{g})$. Como tanto $\Sigma$ quanto $\mathrm{I}(\mathfrak{g})$ são suaves, pela propriedade Cont. de transversalidade que veremos no Teo.29, eles se mantém abertos em uma vizinhança de $p$ em $\Sigma$ e provamos o teorema ${ }^{3}$.

Lembrando que temos um difeomorfismo entre uma vizinhança da seção nula de $\nu N$ e um aberto $U$ de $P$ dado pela aplicação exponencial, nós temos que para $y \in U$, existe um

\footnotetext{
${ }^{3}$ Fica como exercício para o leitor descobrir como nossa construção da faixa de Moebius "torta" não se encaixa nas suposições do teorema.
} 
único $q \in N$ e um único $v \in B_{\delta}\left(0_{q}\right) \subset \nu_{q} N$ tal que $y=\operatorname{Exp}_{q}(v)$. Mas como acabamos de ver, existem também únicos $g \in G$ e $u \in B_{\delta}\left(0_{p}\right) \subset \nu_{p} N$, onde $d g(u)=v$ tal que

$$
y=\operatorname{Exp}_{g \cdot p}(d g(u))=g \cdot \operatorname{Exp}_{p}(u)=g \cdot x
$$

onde $x \in \Sigma_{p}$, portanto, como o grupo $G$ age como grupo de difeomorfismos sobre $P$, nós temos um difeomorfismo

$$
\begin{aligned}
\psi_{p}: & U \longrightarrow \Sigma_{p} \times G \\
y & \longmapsto(x, g)
\end{aligned}
$$

onde $(x, g)$ é o único tal que $y=g \cdot x$.

Suponhamos que temos uma outra seção $\Sigma$ de $P$ sobre $\theta$, i.e.: subvariedade de $P$ contida em $U$ que intercepta em um único ponto as órbitas de $\theta$ e é transversal a elas, portanto $\Sigma$ é de mesma dimensão que $\Sigma_{p}$, e intercepta unicamente também as órbitas de $\Sigma_{p}$. Portanto, para cada $y \in \Sigma$ existe um único elemento $g(y) \in G$ tal que $(g(y) \cdot y) \in \Sigma_{p}$. Logo para $y \in \Sigma$ temos $y=g(y)^{-1} \cdot(g(y) \cdot y)$ e portanto pela propriedade de $\psi_{p}$ nós temos uma única decomposição:

$$
\begin{aligned}
\psi_{p \mid \Sigma}: \Sigma & \longrightarrow \Sigma_{p} \times G \\
y & \longmapsto\left(g(y) \cdot y, g(y)^{-1}\right)
\end{aligned}
$$

Por $\Sigma$ ser subvariedade lisa e $\psi_{p}$ ser também suave, as aplicações $g, g^{-1}: \Sigma \rightarrow G$ são suaves. Portanto $y \mapsto g(y) \cdot y$ é um difeomorfismo entre $\Sigma$ e $\Sigma_{p}$, logo existe também difeomorfismo $U \stackrel{\psi}{\simeq} \Sigma \times G$. Ou seja, nós temos, para $(y, h) \in \Sigma \times G$, o mapa de transição $\psi_{p} \circ \psi^{-1}$ : $\Sigma \times G \longrightarrow \Sigma_{p} \times G$, dado por

$$
\psi_{p} \circ \psi^{-1}(y, h)=\psi_{p}(h \cdot y)=\psi_{p}\left(h g^{-1}(y) g(y) \cdot y\right)=\left(g(y) \cdot y, h g^{-1}(y)\right)
$$

que é uma composição de difeomorfismos sobre a estrutura diferenciável de $P$ e portanto podemos tomar um atlas para $P$ dado por cartas dessa forma.

Como mencionamos, a topologia de $M$ é definida pela projeção ser aberta e contínua. Como existe bijeção entre $\Sigma$ e $U / G=\pi(U)=\theta$, e $\Sigma$ tem a topologia induzida, nós temos um homeomorfismo entre $\Sigma$ e $\theta$ dado por

$$
\begin{array}{r}
\pi_{\mid \Sigma}: \Sigma \longrightarrow \theta \\
\pi_{\mid \Sigma}^{-1}=\operatorname{pr}_{\Sigma} \circ \pi^{-1}: \theta \longrightarrow \Sigma
\end{array}
$$

onde $\operatorname{pr}_{\Sigma}: \Sigma \times G \rightarrow \Sigma$ é a projeção cann̂onica na primeira coordenada. Podemos induzir então estrutura diferenciável em $M$ pela estrutura diferenciável dos $\Sigma$ 's, isto é, tomando $\pi_{\mid \Sigma}$ como difeomorfismo sobre cada $\theta$. Essa estrutura é compatível nas intersecções, isto é, se tivermos um outro aberto $\widetilde{U} \stackrel{\widetilde{\psi}}{\simeq} \widetilde{\Sigma} \times G$, mostramos que sobre a interseção $U \cap \widetilde{U}$ existe um difeomorfismo entre os $\Sigma$ 's, portanto está bem definida a estrutura diferenciável de $M$; existe e é única. É claro que com essa exigência $\pi$ é uma projeção diferenciável e de posto máximo. 
Reciprocamente, se exigirmos que a projeção $\pi: P \rightarrow M$ seja diferenciável e de posto máximo, i.e.: se exigirmos que a estrutura diferenciável de $M$ seja tal que $\pi$ é submersão, obtemos que, dado um ponto qualquer $p \in P$ e uma seção $\Sigma$ que passa por $p$,

$$
d \pi_{p}: T_{p} \Sigma \oplus \mathrm{I}_{p} \mathfrak{g} \longrightarrow T_{\pi(p)} M
$$

mas $\operatorname{dimKer} d \pi_{p}=\operatorname{dim} G=\operatorname{dimg}=\operatorname{dimI}_{p} \mathfrak{g}$ e para $X \in \mathfrak{g}$ nós temos

$$
d \pi_{p}\left(\mathrm{I}_{p}(X)\right)=\left.\frac{d}{d t}\right|_{t=o}(\pi \circ(\exp (t X) \cdot p))=\left.\frac{d}{d t}\right|_{t=o} \pi(p)=0
$$

i.e.: $\mathrm{I}_{p} \mathfrak{g} \subset \operatorname{Ker} d \pi_{p}$ e por dimensão $\operatorname{Ker} d \pi_{p}=\mathrm{I}_{p} \mathfrak{g}$. Portanto $T_{p} \Sigma \stackrel{d \pi_{p}}{\simeq} T_{\pi(p)} M$, e teremos difeomorfismos locais entre as seções e abertos de $M$.

Provamos então:

Theorem 20 Existe uma única estrutura diferenciável em $M$ caracterizada por qualquer uma das condições:

- A projeção $\pi: P \longrightarrow M$ é uma submersão suave.

- Se $\Sigma$ é uma seção de $P$ sobre $\theta$ então $\pi_{\mid \Sigma}$ é um difeomorfismo de $\Sigma$ sobre $\theta$.

Fica claro ainda que $P$ é localmente difeomorfo a conjuntos da forma $\theta \times G$, i.e.: podemos tomar um difemorfismo $\pi^{-1}(\theta) \stackrel{\phi}{\simeq} \theta \times G$ simplesmente aplicando a projeção $\pi_{\mid \Sigma}$ à primeira coordenada do difeomorfismo $\pi^{-1}(\theta) \stackrel{\psi}{\simeq} \Sigma \times G$. Chamaremos tais difeomorfismos de triviliazações locais.

Ademais, podemos considerar uma seção $\Sigma$ sobre $\theta \subset M$ como uma imersão suave que leva $\theta$ na subvariedade $\Sigma, s: \theta \rightarrow P$. Teremos que para $m \in \theta, \pi(s(m))=m$, ou seja, para cada órbita $m \in \theta, s(m)$ está na fibra sobre $m$. Da mesmsa forma se $\tilde{s}$ for outra seção sobre $\theta$, existe um único mapa $g: \theta \rightarrow G$ tal que $\widetilde{s}(m)=g(m) \cdot s(m)$. Chamamos $g$ de mapa de transição entre as seções.

É claro que se $\phi: \pi^{-1}(\theta) \rightarrow \theta \times G$ for uma trivialização local correspondente à subvariedade $\Sigma$, i.e.: à imersão $s: \theta \rightarrow P$, então para todo $m \in \theta, \phi(s(m))=(m, e)$ já que $\psi(s(m))=(s(m), e)$ e $\pi_{\mid \Sigma}(s(m))=m$. Desta forma podemos ver que dada uma seção de $P$ sobre $\theta, s: \theta \rightarrow P$, existe uma única trivialização local $\phi^{s}: \pi^{-1}(\theta) \rightarrow \theta \times G$ a ela adaptada de forma que para todo $m \in \theta$ nós tenhamos $\phi^{s}(s(m))=(m, e)$. Nos casos em que estiver subentendida à qual seção a trivialização está adaptada omitiremos o superscrito " $s$ ".

Nós vimos que para a decomposição $\pi^{-1}(\theta) \stackrel{\psi}{\simeq} \Sigma \times G$, para todo $h \in G$, vale a propriedade

$$
\psi^{-1}(p, h)=h \cdot \psi^{-1}(p, e)=h \cdot p
$$

Para $\pi^{-1}(\theta) \stackrel{\phi}{\simeq} \theta \times G$, para $m \in \theta$ tal que $\pi_{\mid \Sigma}^{-1}(m)=p$, nós temos $\psi^{-1}(p, h)=\phi^{-1}(m, h)$. Logo:

$$
\phi^{-1}(m, h)=h \cdot \phi^{-1}(m, e)
$$

É ainda claro que temos um difeomorfismo

$$
\phi^{-1}(m, \cdot): G \longrightarrow \pi^{-1}(m)
$$


ao qual chamaremos de $\phi_{m}: \pi^{-1}(m) \rightarrow G$ e que, para qualquer $h \in G$ e $p \in \pi^{-1}(m)$, por (2.8) acima obedece:

$$
\phi_{m}(h \cdot p)=h \phi_{m}(p)
$$

onde denotamos o produto no grupo $(h, g) \mapsto h g$. Agora, $\phi_{m}(h \cdot p) \in G$ e $\left(\phi_{m}(h \cdot p)\right)^{-1}=$ $\left(\phi_{m}(p)\right)^{-1} h^{-1}$, logo, se tivermos outro difeomorfismo $\widetilde{\phi}_{m}$ nós temos

$$
\begin{gathered}
\left(\phi_{m}(h \cdot p)\right)^{-1}\left(\widetilde{\phi}_{m}(h \cdot p)\right)=\left(\phi_{m}(p)\right)^{-1} h^{-1} h \widetilde{\phi}_{m}(p)=\left(\phi_{m}(p)\right)^{-1} \widetilde{\phi}_{m}(p) \\
\therefore \quad\left(\phi_{m}(p)\right)^{-1} \widetilde{\phi}_{m}(p)=\left(\phi_{m}(q)\right)^{-1} \widetilde{\phi}_{m}(q)
\end{gathered}
$$

para todos $p, q \in \pi^{-1}(m)$. Isto é, para a transição entre trivializações locais existe um único elemento de $G$ para cada fibra, ou seja, novamente a transição é uma função suave $g_{\phi \widetilde{\phi}}: \theta \rightarrow G$. Uma palavra de esclarecimento em relação ao uso do termo "transição" é aqui necessário, já que não é 'obvia a relação entre $\widetilde{\phi}_{m} \circ \phi_{m}^{-1} \in \operatorname{Aut}(G)$ e $g_{\phi \tilde{\phi}}: \theta \rightarrow G$. Seja então $h \in G$ e $\phi_{m}^{-1}(h)=p$. É claro que $\left(\phi_{m}(p)\right)^{-1}=h^{-1}$. Então obtemos

$$
h^{-1}\left(\widetilde{\phi}_{m} \circ \phi_{m}^{-1}(h)\right)=\left(\phi_{m}(p)\right)^{-1}\left(\widetilde{\phi}_{m}(p)\right)
$$

que como vimos não depende de $p \in \pi^{-1}(m)$. Por isso a função de transição de uma representação à outra é rígida, um único elemento de $G$ para cada ponto de $\theta$.

\subsection{Conexões em Fibrados Principais}

Sobre cada fibra, existe uma maneira canônica de identificar elementos de $T P$, a saber, pelo isomorfismo linear dado pela ação do grupo $G$ sobre a fibra; $d g: T_{p} P \rightarrow T_{g \cdot p} P$. Incorporamos este princípio sempre que exigirmos que alguma estrutura seja $G$-invariante. Contudo sobre elementos de $T P$ que residem sobre diferentes fibras, assim como em fibrados vetoriais, não há identificação canônica. Antecipando um pouco a nomenclatura que segue, se chamarmos o deslocamento sobre as fibras de "vertical", queremos uma forma de identificação puramente " horizontal", uma forma de mantermos uma curva de $P$ "à mesma altura". Apesar deste “ deslocamento vertical" estar canonicamente determinado, não existe na estrutura de $P$ algo que nos dê um complemento, que especifique um " deslocamento horizontal" canônico. Para incorporar uma identificação local em $T P$ ( i.e.: tanto horizontal quanto vertical) precisamos de uma decomposição de $T P$ em subfibrados vertical e horizontal que sejam $G$-invariantes (precisamos manter a identificação canônica sobre as fibras). Como veremos, a escolha de uma conexão nos fornece tal decomposição.

Como $\pi: P \rightarrow M$ é uma submersão, $\operatorname{Ker}(d \pi)$ é um sub-fibrado liso de $T P$, chamado de sub-fibrado vertical, cuja fibra em $p$ denotaremos por $\mathrm{V}_{p}=\mathrm{I}_{p} \mathfrak{g}$. Como $\mathrm{I}_{p} \mathfrak{g}$ é injeção linear,

$$
\mathrm{I}_{p}: \mathfrak{g} \longrightarrow T_{p} P
$$

é um isomorfismo linear de $\mathfrak{g}$ em $\mathrm{V}_{\boldsymbol{p}}$.

Definition 14 Para cada $p \in P$ definimos $\widetilde{\omega}_{p}: V_{p} \rightarrow \mathfrak{g}$ com sendo $I_{p}^{-1}$. 
Desse modo é claro que $\widetilde{\omega}$ é uma 1 -forma suave em $\mathrm{V}$ a valores em $\mathfrak{g}$. Para cada $g \in G$ nós obtemos uma 1-forma em $\mathrm{V}$ a valores em $\mathfrak{g}, g^{*} \widetilde{\omega}$ por

$$
\left(g^{*} \widetilde{\omega}\right)_{p}=\widetilde{\omega}_{g \cdot p} \circ d g
$$

Utilizando 2.1 obtemos

$$
\begin{gathered}
\mathrm{I}_{g \cdot p}=d g \circ \mathrm{I}_{p} \circ \operatorname{Ad}\left(g^{-1}\right) \Rightarrow\left(\mathrm{I}_{g \cdot p}\right)^{-1}=\operatorname{Ad}(g) \mathrm{I}_{p}^{-1} d g^{-1} \\
\therefore \widetilde{\omega}_{g \cdot p} \circ d g=\operatorname{Ad}(g) \circ \widetilde{\omega}_{p}
\end{gathered}
$$

e finalmente

$$
g^{*} \widetilde{\omega}=\operatorname{Ad}(g) \circ \widetilde{\omega}
$$

Definition 15 Uma forma de conexão em $P$ é definida como $\lambda \in \Gamma\left(\Lambda^{1}(T P) \otimes \mathfrak{g}\right)$ que obedeça $g^{*} \lambda=A d(g) \lambda$ e tal que para $u \in V_{p}$ nós tenhamos : $\lambda_{p}(u)=I_{p}^{-1}(u)$.

Em breve discutiremos o significado geométrico de formas de conexão.

Definition 16 Uma conexão em $P$ é um subfibrado $H$ de $T P$ tal que $d g\left(H_{p}\right)=H_{g \cdot p} e$ $H_{p} \oplus V_{p}=T_{p} P$. Chamamos $H$ de fibrado horizontal.

Pela equação (2.1), como a ação de $\operatorname{Ad}(g)$ é automorfismo de $\mathfrak{g}$, nós temos que $d g\left(\mathrm{~V}_{\boldsymbol{p}}\right)=$ $\mathrm{V}_{g \cdot p}$. Portanto a decomposição $T_{p} P=\mathrm{H}_{p} \oplus \mathrm{V}_{p}$ é invariante pela ação de $G$. Denotamos as projeções suaves de $T P$ no subfibrado horizontal de $\widehat{H}$ e no vertical de $\widehat{V}$. Como a decomposição do fibrado $T P$ é invariante pela ação de $G$, é claro que $\widehat{H}_{g \cdot p} \circ d g=d g \circ \widehat{H}_{p}$, o mesmo valendo para a projeção vertical. Explicitamente, se $w \in T_{p} P$ então $w=w_{h}+w_{v} \mathrm{e}$ $d g\left(w_{h}\right) \in \mathrm{H}_{g \cdot p}, d g\left(w_{v}\right) \in \mathrm{V}_{g \cdot p}$, portanto

$$
\begin{gathered}
d g\left(\widehat{H}_{p}(w)\right)=d g\left(w_{h}\right) \text { mas } d g(w)=d g\left(w_{h}\right)+d g\left(w_{v}\right) \\
\therefore \quad \widehat{H}_{g \cdot p} d g(w)=\widehat{H}_{g \cdot p} d g\left(w_{h}\right)=d g\left(w_{h}\right)
\end{gathered}
$$

Theorem 21 Se $H$ é conexão em $P$, então para todo $p \in P: d \pi_{p}: T_{p} P \rightarrow T_{\pi(p)} M$ se restringe a um isomorfismo linear $h_{p}: H_{p} \rightarrow T_{\pi(p)} M$ para o qual vale $\left.h_{g \cdot p} \circ d g\right|_{H_{p}}=h_{p}$.

Dem:Que a projeção se restringe a um isomorfismo linear é claro, já que o espaço vertical é o núcleo da projeção, que tem posto máximo, e o horizontal é seu complemento. Agora nós temos que $h_{g \cdot p} \circ \widehat{H}_{g \cdot p}=d \pi_{g \cdot p}$, logo, aplicando os dois lados a $d g$ obtemos:

$$
\begin{gathered}
h_{g \cdot p} \circ \widehat{H}_{g \cdot p} \circ d g=h_{g \cdot p} \circ d g \circ \widehat{H}_{p} \\
=d \pi_{g \cdot p} \circ d g=d\left(\pi_{g \cdot p} \circ g\right)=d \pi_{p}=h_{p} \circ \widehat{H}_{p} \\
\therefore h_{p} \circ \widehat{H}_{p}=h_{g \cdot p} \circ d g \circ \widehat{H}_{p}
\end{gathered}
$$

e como $\widehat{H}_{p}$ é sobrejetor, obtemos o enunciado.

Definition 17 Se H é a conexão em $P$ definimos a 1-forma de conexão como $\omega:=\widetilde{\omega} \circ \widehat{V}$. Isto é :

$$
\begin{aligned}
\omega_{p}: T_{p} P & \longrightarrow \mathfrak{g} \\
u & \longrightarrow I_{p}^{-1} \circ \widehat{V}_{p}(u)
\end{aligned}
$$


É claro que se $v \in \mathrm{V}_{p}$ então $\omega(v)=\widetilde{\omega}(v)$. Além disso

$$
\begin{gathered}
\left(g^{*} \omega\right)_{p}=\omega_{g \cdot p} \circ d g=\widetilde{\omega}_{g \cdot p} \circ \widehat{V}_{g \cdot p} \circ d g \\
=\widetilde{\omega}_{g \cdot p} \circ d g \circ \widehat{V}_{p}=\left(g^{*} \widetilde{\omega}\right)_{p} \circ \widehat{V}_{p}=\operatorname{Ad}(g) \circ \widetilde{\omega}_{p} \circ \widehat{V}_{p}=\operatorname{Ad}(g) \omega_{p}
\end{gathered}
$$

e portanto obtemos

$$
g^{*} \omega=\operatorname{Ad}(g) \omega
$$

Portanto $\omega$ realmente é forma de conexão. Como $\mathrm{I}_{p}$ é isomorfismo linear sobre $\mathrm{V}_{p}$, é claro que $\operatorname{Ker} \omega_{p}$ é complementar a $\mathrm{V}_{p}$. Além disso, se $u \in \operatorname{Ker} \omega_{p}$ então $d g(u) \in \operatorname{Ker} \omega_{g \cdot p}$ já que

$$
\omega_{g \cdot p} \circ d g(u)=\operatorname{Ad}(g) \omega_{p}(u)=0
$$

logo, como $d g$ é isomorfismo linear, Ker $\omega$ é um subfibrado $G$-invariante de $T P$ complementar a V. Ou seja, podemos definir uma conexão em $P$ como $\mathrm{H}_{p}=\operatorname{Ker} \omega_{p}$. De fato temos:

Theorem 22 O mapa $H \rightarrow \omega$ é uma correspondência bijetora.

Dem: Como $I_{p}: \mathfrak{g} \rightarrow V_{p}$ é isomorfismo linear, identificamos em cada ponto $\omega$ como a projeção no subespaço $V_{p}$. Como qualquer projeção, $\omega$ será caracterizada por seu núcleo, $H$, ou mais precisamente, por uma decomposição $T_{p} P=\mathrm{H}_{p} \oplus \mathrm{V}_{p}$. Já demonstramos a afirmação inversa.

Essa é uma forma geométrica de encarar uma forma de conexão; simplesmente como uma projeção em um subfibrado $G$-invariante de $T P$, complementar ao espaço tangente às órbitas.

Para cada seção $s: \theta \rightarrow P$ e forma de conexão $\omega$ em $P$, definimos a 1-forma em $\theta$ a valores em $\mathfrak{g}$ :

$$
\omega^{s}:=s^{*} \omega
$$

ou seja, se $u \in T_{m} M$ então $\omega_{m}^{s}(u)=\omega_{s(m)}\left(d s_{m}(u)\right)$.

Proposition 15 Se $s$ e $\widetilde{s}$ são seções sobre $\theta, g: \theta \rightarrow G$ é o mapa de transição, e $\omega$ é uma 1-forma de conexão em $P$, então

$$
\omega^{\bar{s}}=A d(g) \circ \omega^{s}+(d g) g^{-1}
$$

Dem: Por definição nós temos, para $m \in \theta, u \in T_{m} M$,

$$
\omega_{m}^{\tilde{s}}(u)=\omega_{\tilde{s}(m)}(d \tilde{s}(u))=\omega_{g(m) \cdot p}(d \tilde{s}(u))
$$

Como $g: \theta \rightarrow G$, se $m \in \theta$ nós temos $d g_{m}: T_{m} M \rightarrow T_{g(m)} G$, um sentido diferente do que estávamos usando: $d g: T P \rightarrow T P$, que leva um vetor tangente a uma curva $\alpha(t)$ passando por $p \in P$ à tangente à curva $g \cdot \alpha(t)$ passando por $g \cdot p$. Portanto, denotaremos essa última aplicação, dado $m \in \theta$, por $L_{g(m)_{*}}: T P \rightarrow T P$, i.e.: utilizaremos a notação comum de $L_{g}$ como multiplicação à esquerda e $R_{g}$ como multiplicação à direita . Agora, por (2.10)

$$
\omega_{g(m) \cdot p} \circ L_{g(m)_{*}}=\left(L_{g(m)}^{*} \omega\right)_{p}=\operatorname{Ad}(g(m)) \omega_{p}
$$


Novamente denotando a ação do grupo em $P$ por $\mu: P \times G \rightarrow P$, temos $\tilde{s}=\mu(s, g)$ e então $d \tilde{s}=d \mu(d s, d g)$. Aplicando (2.13) a $L_{g(m)^{-1}}$ em ambos os lados e colocando em (2.12) obtemos:

$$
\omega_{m}^{\tilde{s}}(u)=\operatorname{Ad}(g(m)) \omega_{p} \circ L_{g(m)^{-1} *} d \mu_{(s(m), g(m))}\left(d s_{m}(u), d g_{m}(u)\right)
$$

Agora utilizando a trivialização local adaptada a $s$, dada por $\pi^{-1}(\theta) \stackrel{\phi^{s}}{\simeq} \theta \times G$ nós temos, utilizando (2.8)

$$
\begin{array}{r}
\mu(s(m), g(m))=\left(\phi^{s}\right)^{-1}(m, g(m)) \Longrightarrow \mu(s, g)=\left(\phi^{s}\right)^{-1}\left(\operatorname{Id}_{\theta}, g\right) \\
\therefore \quad d \mu(d s, d g)=\left(d \phi^{s}\right)^{-1}\left(\operatorname{Id}_{\left.T M\right|_{\theta}}, d g\right) \\
\therefore \quad d \mu_{(s(m), g(m))}\left(d s_{m}(u), d g_{m}(u)\right)=\left(d \phi^{s}\right)_{(m, g(m))}^{-1}\left(u, d g_{m}(u)\right)
\end{array}
$$

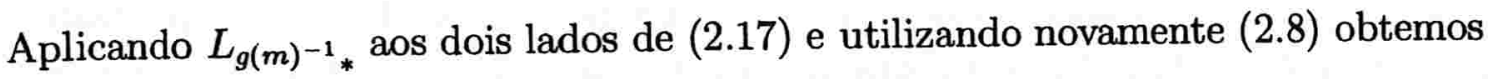

$$
\begin{gathered}
L_{g(m)^{-1} *} d \mu_{(s(m), g(m))}\left(d s_{m}(u), d g_{m}(u)\right)=L_{g(m)^{-1} *}\left(d \phi^{s}\right)_{(m, g(m))}^{-1}\left(u, d g_{m}(u)\right) \\
=\left(d \phi^{s}\right)_{(m, e)}^{-1}\left(u, L_{g(m)^{-1} *}\left(d g_{m}(u)\right)\right)=\left(d \phi^{s}\right)_{(m, e)}^{-1}(u, 0)+\left(d \phi^{s}\right)_{(m, e)}^{-1}\left(0, L_{g(m)^{-1} *}\left(d g_{m}(u)\right)\right)
\end{gathered}
$$

Agora $\left(d \phi^{s}\right)_{(m, e)}^{-1}(u, 0)=d s_{m}(u)$ já que se, para $t=0, \alpha: I \rightarrow \theta$ é curva tangente a $u$ em $m \in \theta$ :

$$
\left(d \phi^{s}\right)_{(m, e)}^{-1}(u, 0)=\left.\frac{d}{d t}\right|_{t=o}\left(\left(\phi^{s}\right)^{-1}(\alpha(t), e)\right)=\left.\frac{d}{d t}\right|_{t=o} s(\alpha(t))=d s_{m}(u)
$$

Além disso, para qualquer $X \in \mathfrak{g}$ nós temos

$$
\begin{gathered}
\left(d \phi^{s}\right)_{(m, e)}^{-1}(0, X)=\left.\frac{d}{d t}\right|_{t=o}\left(\left(\phi^{s}\right)^{-1}(m, \exp t X)\right)=\left.\frac{d}{d t}\right|_{t=o} \quad(\exp t X \cdot s(m))=\mathrm{I}_{s(m)} X \\
\therefore \quad\left(d \phi^{s}\right)_{(m, e)}^{-1}\left(0, L_{g(m)^{-1} *}\left(d g_{m}(u)\right)\right)=\mathrm{I}_{s(m)}\left(L_{g(m)^{-1} *}\left(d g_{m}(u)\right)\right)
\end{gathered}
$$

Utilizando (2.19) e (2.14) obtemos finalmente:

$$
\begin{gathered}
\omega_{p} \circ L_{g(m)^{-1} *} d \mu_{(s(m), g(m))}\left(d s_{m}(u), d g_{m}(u)\right)=\omega_{p}\left(d s_{m}(u)\right)+L_{g(m)^{-1} *}\left(d g_{m}(u)\right) \\
\therefore \quad \omega_{m}^{\tilde{s}}(u)=\operatorname{Ad}(g(m)) \omega_{p}\left(d s_{m}(u)\right)+R_{g(m)^{-1}{ }^{*}} L_{g(m)_{*}} L_{g(m)^{-1} *}\left(d g_{m}(u)\right) \\
\therefore \quad \omega_{m}^{\tilde{s}}(u)=\operatorname{Ad}(g(m)) \omega_{p}^{s}(u)+R_{g(m)^{-1} *} d g_{m}(u)
\end{gathered}
$$

Proposition 16 Recíprocamente, se para cada seção $s$ de $P$ sobre $\theta$ nós tivermos uma 1forma sobre $\theta$ a valores em $\mathfrak{g}, \omega^{s}$, e se essas 1-formas satisfizerem (2.11), então existe uma única forma de conexão $\omega$ em $P$ tal que $\omega^{s}=s^{*} \omega$.

Dem: Seja $w \in T_{p} P, p=s(m) \in P$, e $\phi: \pi^{-1}(\theta) \rightarrow \theta \times G$ a trivialização local adaptada ${ }^{4}$ à seção $s$. Então, para cada $w \in T_{p} P$, existem únicos $u \in T_{m} M, X \in \mathfrak{g}$ tal que

\footnotetext{
${ }^{4}$ Abreviamos aqui $\phi^{s}$ por $\phi$.
} 


$$
w=(d \phi)_{(m, e)}^{-1}(u, X)=d s_{m}(u)+\mathrm{I}_{s(m)} X
$$

Definimos então $\omega \in \Gamma\left(\Lambda^{1}\left(T P_{\mid \theta}\right) \otimes \mathfrak{g}\right)$ como

$$
\omega_{p}(w)=\omega_{p}\left((d \phi)_{(m, e)}^{-1}(u, X)\right):=\omega_{m}^{s}(u)+X
$$

Como sabemos, para todo $y \in \pi^{-1}(\theta)$ existem únicos $x \in \Sigma=s(\theta)$ e $h \in G$ tal que $y=h \cdot x$. Logo para $v \in T_{y} P$ estendemos a definição de $\omega$ :

$$
\omega_{y}(v)=\omega_{h \cdot x}(v):=\operatorname{Ad}(h) \omega_{x}\left(L_{h^{-1} *}(v)\right)
$$

Precisamos mostrar que se $\omega^{s}$ obedece (2.11), essa definição independe das seções que tomarmos; i.e.: a forma $\omega$ em $P$ relativa à seção $s$ é igual a forma $\widetilde{\omega}$ obtida pela definição a partir da seção $\tilde{s}=g \cdot s$. Claramente, se estiver bem definida, $h^{*} \omega=\operatorname{Ad}(h) \omega$, o que pode ser facilmente visto colocando $\tilde{v} \in T_{x} P$ tal que $L_{h_{*}}(\tilde{v})=v$ em (2.27). Além disso, se $v \in \mathrm{V}_{p}$,

$$
v=(d \phi)_{(m, e)}^{-1}(0, X)=\mathrm{I}_{s(m)} X
$$

e portanto pela nossa definição:

$$
\omega_{p}(v)=\omega_{p}\left(\mathrm{I}_{p}(X)\right)=X=\mathrm{I}_{p}^{-1}\left(\mathrm{I}_{p}(X)\right)=\mathrm{I}_{p}^{-1}(v) .
$$

portanto, se estiver bem definida, $\omega$ é forma de conexão. Seja então $\tilde{\phi}: \pi^{-1}(\theta) \rightarrow \theta \times G$ uma trivialização local adaptada à seção $\tilde{s}=g \cdot s$. Como $L_{g(m)^{-1}{ }_{*}}$ é isomorfismo linear para todo $m \in \theta$, existem únicos $u \in T_{m} M, X \in \mathfrak{g}$ tal que

$$
w=L_{g(m)^{-1} *}\left(d \tilde{\phi}_{(m, e)}^{-1}(u, X)\right)
$$

onde como mencionamos $w \in T_{p} P$ e portanto $d \tilde{\phi}_{(m, e)}^{-1}(u, X) \in T_{g(m) \cdot p} P$. Mas

$$
d \tilde{\phi}_{(m, e)}^{-1}(u, 0)=d \tilde{s}_{m}(u)
$$

e como vimos no exercício anterior

$$
L_{g(m)^{-1}{ }_{*}}\left(d \tilde{s}_{m}(u)\right)=d s_{m}(u)+\mathrm{I}_{p}\left(L_{g(m)^{-1}{ }_{*}}\left(d g_{m}(u)\right)\right)
$$

e utilizando (2.20):

$$
L_{g(m)^{-1} *}\left(d \tilde{\phi}_{(m, e)}^{-1}(0, X)\right)=L_{g(m)^{-1} *} \mathrm{I}_{g(m) \cdot p}(X)
$$

Mas por (2.1) obtemos

$$
L_{g(m)^{-1} *}\left(\mathrm{I}_{g(m) \cdot p}(X)\right)=L_{g(m)^{-1} *}\left(L_{g(m)_{*}}\left(\mathrm{I}_{p}\left(\operatorname{Ad}\left(g(m)^{-1}\right) X\right)\right)\right)=\mathrm{I}_{p}\left(\operatorname{Ad}\left(g(m)^{-1}\right) X\right)
$$

Agora, por (2.29) e (2.31) obtemos

$$
\begin{aligned}
w & =d s_{m}(u)+\mathrm{I}_{p}\left(L_{g(m)^{-1} *}\left(d g_{m}(u)\right)+\operatorname{Ad}\left(g(m)^{-1}\right) X\right) \\
& =(d \phi)_{(m, e)}^{-1}\left(u, L_{g(m)^{-1} *}\left(d g_{m}(u)\right)+\operatorname{Ad}\left(g(m)^{-1}\right) X\right) \\
\therefore \quad \omega_{p}(w) & =\omega_{m}^{s}(u)+L_{g(m)^{-1} *}\left(d g_{m}(u)\right)+\operatorname{Ad}\left(g(m)^{-1}\right) X
\end{aligned}
$$


Por outro lado, utilizando as equações (2.27) e (2.28):

$$
\begin{aligned}
\widetilde{\omega}_{p}(w) & =\widetilde{\omega}_{g(m)^{-1} \cdot \tilde{s}(m)}(w) \\
& =\operatorname{Ad}\left(g(m)^{-1}\right) \widetilde{\omega}_{\tilde{s}(m)}\left(L_{g(m)_{*}}(w)\right) \\
& =\operatorname{Ad}\left(g(m)^{-1}\right) \widetilde{\omega}_{\tilde{s}(m)}\left(L_{g(m)_{*}}\left(L_{g(m)^{-1} *}\left(d \tilde{\phi}_{(m, e)}^{-1}(u, X)\right)\right)\right) \\
& =\operatorname{Ad}\left(g(m)^{-1}\right) \widetilde{\omega}_{\tilde{s}(m)}\left(d \tilde{\phi}_{(m, e)}^{-1}(u, X)\right) \\
& =\operatorname{Ad}\left(g(m)^{-1}\right)\left(\omega_{m}^{\tilde{s}}(u)+X\right)
\end{aligned}
$$

Finalmente aplicando a regra de transformação (2.24) a (2.39) obtemos:

$$
\begin{aligned}
\tilde{\omega}_{p}(w) & =\operatorname{Ad}\left(g(m)^{-1}\right)\left(\operatorname{Ad}(g(m)) \omega_{p}^{s}(u)+R_{g(m)^{-1} *} d g_{m}(u)+X\right) \\
& =\omega_{p}^{s}(u)+L_{g(m)^{-1} *}\left(d g_{m}(u)\right)+\operatorname{Ad}\left(g(m)^{-1}\right) X
\end{aligned}
$$

e portanto obtemos a igualdade almejada. Demonstrar unicidade da forma de conexão é bem mais fácil. Se $\omega^{\prime}$ é outra forma de conexão, tal que para toda seção $s, s^{*} \omega=s^{*} \omega^{\prime}$, elas claramente concordam sobre vetores da forma $d s_{m}(u)=d \phi_{(m, e)}^{-1}(u, 0)$. Por outro lado sobre vetores verticais, $v=d \phi_{(m, e)}^{-1}(0, X)$ nós temos que $\omega(v)=\omega^{\prime}(v)=\mathrm{I}_{p}^{-1}(v)=X$. Como $d \phi_{p}$ é isomorfismo, $\omega=\omega^{\prime}$.

\section{Curvatura em Fibrados Principais}

Definition 18 Se $\omega \in \Gamma\left(\Lambda^{1}(T P) \otimes \mathfrak{g}\right)$ é forma de conexão definimos a curvatura de $\omega$, $\Omega \in \Gamma\left(\Lambda^{2}(T P) \otimes \mathfrak{g}\right)$ por

$$
\Omega:=d \omega+\omega \wedge \omega
$$

Lembramos que $d$ é a derivada exterior e que estamos utilizando a aplicação da álgebra de Lie assim como em (1.33), i.e.: para $X_{1}, X_{2} \in \Gamma(T P)$,

$$
\omega \wedge \omega\left(X_{1}, X_{2}\right)=\frac{1}{2}\left(\omega\left(X_{1}\right) \omega\left(X_{2}\right)-\omega\left(X_{2}\right) \omega\left(X_{1}\right)\right)=\left[\omega\left(X_{1}\right), \omega\left(X_{2}\right)\right]
$$

Theorem 23 Se $H_{p}=K e r \omega_{p}$ então $d \omega \circ \widehat{H}=\Omega$.

Dem: Queremos provar que $d \omega(\widehat{H} X, \widehat{H} Y)=\Omega(X, Y)$ para quaisquer $X, Y \in \Gamma(T P)$. Como já foi demonstrado no Cap.I ambos os lados são bilineares e anti-simétricos. Logo nos basta verificar a afirmação para três casos:

- $X, Y \in \mathrm{V}$ : Nós temos, ainda no caso geral,

$$
\begin{gathered}
\Omega(X, Y)=d \omega(X, Y)+[\omega(X), \omega(Y)] \\
d \omega(X, Y)=Y[\omega(X)]-X[\omega(Y)]-\omega([X, Y])
\end{gathered}
$$


Neste caso específico claramente $d \omega(\widehat{H} X, \widehat{H} Y)=0$. Se $X$ é vertical, então, $X_{p}=\mathrm{I}_{p}(\widetilde{X})$ para algum $\widetilde{X} \in \mathfrak{g}$. Logo, como $d \omega$ é tensorial, $d \omega(X, Y)_{p}$ só depende dos valores dos campos no ponto $p \in P$, portanto

$$
d \omega(X, Y)_{p}=d \omega(\mathrm{I}(\tilde{X}), \mathrm{I}(\widetilde{Y}))_{p}
$$

para $\tilde{X}, \tilde{Y} \in \mathfrak{g}$ apropriados. Portanto, $\omega_{p}(X)=\mathrm{I}_{p}^{-1}(X)=\widetilde{X}$, é um elemento fixo da álgebra de Lie, é um vetor constante, $\operatorname{logo} Y_{p}[\omega(X)]=0$, o mesmo valendo trocando-se $X$ por $Y$, e obtemos então:

$$
d \omega(X, Y)_{p}=Y_{p}[\omega(X)]-X_{p}[\omega(Y)]-\omega_{p}([X, Y])=-\omega_{p}([X, Y])
$$

Agora, assumindo a identidade $[22][\mathrm{I}(\tilde{X}), \mathrm{I}(\widetilde{Y})]=\mathrm{I}([\tilde{X}, \tilde{Y}])$, obtemos

$$
\omega_{p}([X, Y])=\omega_{p}([\mathrm{I}(\tilde{X}), \mathrm{I}(\tilde{Y})])=\omega_{p}\left(\mathrm{I}_{p}([\tilde{X}, \tilde{Y}])\right)=[\tilde{X}, \tilde{Y}]=\left[\omega_{p}(X), \omega_{p}(Y)\right]
$$

E pela equação (2.40) obtemos $\Omega(X, Y)=0$.

- $X \in \mathrm{V}, Y \in \mathrm{H}$ : Novamente é claro que $d \omega(\widehat{H} X, \widehat{H} Y)=0$. Agora $\omega(Y)=0$ e portanto $\Omega(X, Y)=d \omega(X, Y)$. Utilizando o mesmo argumento do item anterior obtemos novamente de (2.41):

$$
d \omega(X, Y)=-\omega([X, Y])
$$

Mas se $X$ é vertical, $X$ é um campo tangente ao fluxo de $g(t)$ para algum $g: I \rightarrow G$. Lembrando que

$$
[X, Y]=\lim _{t \rightarrow 0} \frac{1}{t}\left(L_{g(t)^{-1} *} Y-Y\right)
$$

lembrando que $Y$ é horizontal, e o subfibrado horizontal é $G$-invariante nós temos que $[X, Y] \in$ H. Logo $\omega([X, Y])=0$.

- $X, Y \in \mathrm{H}$ : Nesse caso $d \omega(\widehat{H} X, \widehat{H} Y)=d \omega(X, Y)$. Como $\omega(X)=0$, de (2.40) obtemos também $\Omega(X, Y)=d \omega(X, Y)=-\omega([X, Y])$.

É interessante notar que a forma de curvatura é nula se qualquer um dos campos for vertical, logo os campos relevantes em $T P$ serão aqueles levados de $M$ por alguma seção $s$, podemos então considerar $\Omega$ como uma 2-forma em $T M$ a valores em $\mathfrak{g}$. A forma de curvatura de fibrados principais mede a falta de integrabilidade da distribuição horizontal em ser integrável, em analogia com a forma de curvatura de fibrados vetoriais, que "mede o quanto o mapa $X \longrightarrow \nabla_{X}$ falha em ser homomorfismo de álgebras de Lie". A analogia com o teorema de Frobenius é clara, e merece ser destrinchada.

\section{Conexões Planas}

Diremos que uma conexão em um fibrado principal é plana ou flat se ao redor de cada ponto $p \in P$ existir uma seção $s: \theta \rightarrow \pi^{-1}(\theta)$ para a qual $p \in s(\theta)$ e tal que, para todo $q \in s(\theta)$ 
nós tenhamos um isomorfismo linear: $T_{x} M \stackrel{d s_{x}}{\simeq} \mathrm{H}_{q}$, onde $\pi(q)=x$. Colocado de outra forma, uma conexão é flat se existe uma trivialização local $\phi: \pi^{-1}(\theta) \rightarrow \theta \times G$ tal que para todo $q \in s(\theta)$

$$
\mathrm{H}_{q}=d \phi_{(x, e)}^{-1}\left(T_{x} M \times\{0\}\right)
$$

Como $\mathrm{H}_{g \cdot q}=L_{g_{*}} \mathrm{H}_{q}$, por (2.8)

$$
\mathrm{H}_{g \cdot q}=d \phi_{(x, g)}^{-1}\left(T_{x} M \times\{0\}\right)
$$

portanto podemos redefinir

Definition 19 Uma conexão em um fibrado principal é plana ou flat se ao redor de cada ponto $p \in P$ existir um aberto $\pi^{-1}(\theta)$ e trivialização local $\phi: \pi^{-1}(\theta) \rightarrow \theta \times G$ tal que para todo $q \in \pi^{-1}(\theta)$ existe $g \in G$ para o qual

$$
H_{q}=d \phi_{(\pi(q), g)}^{-1}\left(T_{x} M \times\{0\}\right)
$$

Proposition 17 Uma conexão em $P$ é flat se e somente se a forma de curvatura é nula.

Dem: Chamaremos a restrição de $\phi$ para um $g \in G$ fixo de $\phi_{g}^{-1}: \theta \rightarrow \pi^{-1}(\theta)$. Suponhamos que a conexão seja flat. Nós temos que para todo $x \in \theta$,

$$
\mathrm{H}_{\phi^{-1}(x, g)}=d \phi_{(x, g)}^{-1}\left(T_{x} M \times\{0\}\right)
$$

ou seja

$$
\mathrm{H}_{\phi_{g}^{-1}(x)}=\left(d \phi_{g}^{-1}\right)_{x}\left(T_{x} M\right)
$$

o que por definição significa que para cada $g \in G, \phi^{-1}(\theta \times\{g\})=\phi_{g}^{-1}(\theta)$ é variedade integral da distribuição suave $\mathrm{H}$. Mas se $X, Y \in \mathrm{H}$, então existem $\widetilde{X}, \tilde{Y} \in \Gamma\left(\left.T M\right|_{\theta}\right)$ tais que $d \phi_{g}^{-1}(\widetilde{X})=X \circ \phi_{g}^{-1}$, o mesmo valendo para $\widetilde{Y}$, ou seja, estes campos são $\phi_{g}^{-1}$-relacionados. Portanto

$$
d \phi_{g}^{-1}([\tilde{X}, \tilde{Y}])=[X, Y] \circ \phi_{g}^{-1}
$$

então, como para todo $q \in \pi^{-1}(\theta)$, existem $x \in \theta$ e $g \in G$ tais que $\phi_{g}^{-1}(x)=q$, nós temos

$$
d \phi_{g}^{-1}\left([\tilde{X}, \tilde{Y}]_{x}\right)=[X, Y]_{q}
$$

e portanto por hipótese:

$$
[X, Y]_{q} \in \mathrm{H}_{q}
$$

o que implica que mesmo se $X, Y \in \mathrm{H}, \Omega(X, Y)=-\omega([X, Y])=0$, o que implica por sua vez que $\Omega=0$.

Se por outro lado, supusermos que a forma de curvatura é nula, nós obtemos de cara que a distribuição horizontal é integrável. Chamemos de $\Sigma^{\prime}$ uma variedade integral de $H$ passando por $p \in P$. Como $\mathrm{H} \oplus \mathrm{V}=T P$, para todo $q \in \Sigma$ obtemos que $T_{q} \Sigma^{\prime} \oplus \mathrm{V}_{q}=T_{q} P$, ou seja, $\Sigma^{\prime}$ é transversal ao subfibrado vertical. Agora, nós sabemos que $d \pi_{q}: T_{q} P \rightarrow T_{\pi(q)} M$ é submersão, $\operatorname{logo}$, como $\operatorname{Ker} d \pi_{p}=\mathrm{V}_{p}$, nós temos que $d \pi_{p}: T_{p} \Sigma \rightarrow T_{\pi(p)} M$ é isomorfismo linear. Portanto pelo teorema da função inversa, existe aberto $\Sigma$ de $\Sigma^{\prime}$ que é levado difeomorficamente por $\pi_{\mid \Sigma}$ em um aberto $\theta$ de $M$. Então não podemos ter dois pontos distintos de $\Sigma$ sendo levados 
em um único ponto de $M$, o que signifca que todos os pontos de $\Sigma$ intersectam as órbitas uma única vez. Portanto nós obtemos que $\Sigma$ é uma seção de $P$ sobre $\theta$, e também é uma variedade integral de H. Por ser seção, existe trivialização local $\phi$ tal que para algum $g \in G$, $\phi^{-1}(\theta \times\{g\})=\Sigma$, como $\Sigma$ é variedade integral de $\mathrm{H}$ obtemos o enunciado. Esse teorema significa que a curvatura só é nula se existe algum referencial (ou sistema de coordenadas) em que não se observa efeitos de curvatura (o que poderia ser chamado de referncial euclidiano, numa generalização da nomenclatura de $T M$ ), o que pode ainda ser considerada como outra faceta de seu caráter tensorial.

\section{Levantamento Horizontal}

Uma construção bastante utilizada em fibrados principais é a de levantamento horizontal de campos e curvas. Dizemos que um campo $\widetilde{X} \in \Gamma(T P)$ é o levantamento horizontal de $X \in \Gamma(T M)$ se $\widetilde{X} \in \mathrm{H}$ e para todo $p \in P, d \pi_{p} \widetilde{X}=X_{\pi(p)}$. A existência e unicidade de $\widetilde{X}$ é clara pela existência do isomorfismo linear $d \pi_{p}: \mathrm{H}_{p} \rightarrow T_{\pi(p)} M$. Que o levantamento é suave pode ser visto utilizando o fato que $\pi$ é submersão, portanto existe um campo suave $Y \in \Gamma(T P)$ que se projeta em $X \in \Gamma(T M)$, portanto sua componente horizontal é suave e tem a propriedade desejada. A invariância do campo $\widetilde{X}$ pela ação de $G$ é clara pela invariância de $\mathrm{H}$ e unicidade de $\widetilde{X}$.

Podemos também tomar levantamento horizontal de curvas em $M$, o que pode ser demonstrado simplesmente tomando as curvas integrais do levantamento horizontal de $\gamma^{\prime}$.

Outra forma de demonstração é supor que $\alpha: I \rightarrow \theta \subset M$ é uma curva suave tal que $\alpha(0)=x \in \theta$, então, chamando uma dada trivialização de $\phi: \pi^{-1}(\theta) \rightarrow \theta \times G$, qualquer curva da forma $\gamma(t)=\phi^{-1}(\alpha(t), g(\alpha(t)))$ onde $g: \theta \rightarrow G$ é curva suave tal que $g(\alpha(0))=e$, será um levantamento de $\alpha$ passando por $p$ em $t=0$. Agora,

$$
\gamma^{\prime}(t)=(d \phi)_{(\alpha(t), g(\alpha(t)))}^{-1}\left(\alpha^{\prime}(t), d g_{\alpha(t)} \alpha^{\prime}(t)\right)
$$

e portanto utilizando (2.19), (2.20) e (2.22), nós temos :

$$
\omega_{\gamma(t)}\left(L_{g(\alpha(t))^{-1} *}\left(\gamma^{\prime}(t)\right)\right)=\omega_{\gamma(t)}\left(d s_{\alpha(t)}\left(\alpha^{\prime}(t)\right)\right)+L_{g(\alpha(t))^{-1} *}\left(d g_{\alpha(t)}\left(\alpha^{\prime}(t)\right)\right)
$$

Agora, nós sabemos que $\gamma^{\prime}(t)$ é horizontal se e somente se $L_{g(\alpha(t))^{-1}}\left(\gamma^{\prime}(t)\right)$ for horizontal. Como $\omega_{\gamma(t)}\left(d s_{\alpha(t)}\left(\alpha^{\prime}(t)\right)\right)=\xi(t)$ é uma curva em $\mathfrak{g}$, escrevendo $g(\alpha(t))=g(t)$, i.e.: $L_{g(\alpha(t))^{-1} *}\left(d g_{\alpha(t)}\left(\alpha^{\prime}(t)\right)\right)=g(t)^{-1} g^{\prime}(t)$, obtemos finalmente que $\gamma(t)$ é horizontal se existe solução, para a equação

$$
g(t)^{-1} g^{\prime}(t)=\xi(t)
$$

onde $g(0)=e$. Ou seja, justamente a equação (1.108), cuja solução existe e é única pelo Lema 1. Portanto chegamos ao resultado de que se $\alpha(t)$ é uma curva suave em $M$ passando por $x=\alpha(0)$, dado um ponto $p \in P$ tal que $\pi(p)=x$, existe um único levantamento horizontal de $\alpha(t)$ que passa por $p$ em $t=0$, que chamaremos de $\widetilde{\alpha}_{p}(t)$. Por unicidade, e novamente, como o subespaço horizontal é invariante por $G$, nós temos que $g \cdot\left(\widetilde{\alpha}_{p}\right)=\widetilde{\alpha}_{g \cdot p}$. 


\subsection{Fibrado dos Referenciais}

Como mencionamos ao início do capítulo, o conjunto de bases $G$-admissíveis de um fibrado vetorial $E$ serviu de motivação para a introdução de fibrados principais. Verifiquemos então que este conjunto é de fato um $G$-fibrado principal. O conjunto das bases do $k$-fibrado vetorial $E G$-admissíveis, $P(E)$ é dado por $\bigcup_{M} P(E)_{x}$ onde $P(E)_{x}$ é o conjunto de todas as bases admissíveis de $E_{x}$. Ou seja $P(E)=\left\{(x, s(x)) \mid x \in M, s(x)\right.$ base admissível de $\left.E_{x}\right\}$. Aqui a projeção leva simplesmente $(x, s(x)) \mapsto x$. Como vimos ao início do capítulo, existe bijeção entre $G$ e $P(E)_{x}$ para todo $x \in M$, já que dadas duas base admissíveis existe um único elemento de $G \subset G L(k)$ que leva uma na outra. Tomando uma seção lisa de bases sobre $E_{\mid \theta}$, i.e.: $s: x \mapsto s(x) \in P(E)_{x}$, definida por $n$ seções lisas linearmente independentes de $E_{\mid \theta}$, e que chamaremos de agora em diante de referencial, temos a bijeção

$$
\begin{aligned}
\phi^{s}: \theta \times G & \rightarrow P(E)_{\mid \theta} \\
(x, h) & \longmapsto h(s(x))
\end{aligned}
$$

Que é sobrejetor é claro, para mostrar que é também injetora, basta notar que, já que as fibras são disjuntas, se $x \neq y$ não existe $h \in G$ tal que $h(s(x))=s(y)$, e se $x=y$ nós utilizamos o fato que $G$ age livremente (injetoramente) sobre cada fibra. Com essa bijeção induzimos uma estrutura diferenciável em em $P(E)_{\mid \theta}$ pela estrutura diferenciável em $\theta \times G$. Consequentemente um mapa $f: P(E)_{\mid \theta} \rightarrow P(E)_{\mid \theta}$ será suave se e somente $\operatorname{se}^{5} \tilde{f}=\phi^{-1} \circ f \circ \phi$ for suave.

$$
\begin{array}{rll}
P(E)_{\mid \theta} & \stackrel{f}{\longrightarrow} & P(E)_{\mid \theta} \\
\phi \uparrow & & \downarrow \phi^{-1} \\
P(E)_{\mid \theta} & \underset{\tilde{f}}{\longrightarrow} & P(E)_{\mid \theta}
\end{array}
$$

Examinemos a ação do grupo $G$ sobre $P(E)_{\mid \theta}$. Seja $p \in P(E)_{\mid \theta}$, uma base sobre $x$ tal que $p=h_{0}(s(x))=\phi\left(h_{0}, x\right)$, então, fazendo $f=h \in G$, temos

$$
h(p)=h\left(h_{0}(s(x))\right)=\phi\left(x, h h_{0}\right)
$$

mas então $\tilde{h}:\left(x, h_{0}\right) \mapsto\left(x, h h_{0}\right)$, e como a ação $G \times G \rightarrow G$ é suave, a ação de $\tilde{h}$ é suave e consequentemente a a ação de $h$ é suave. Logo o grupo age suave e livremente sobre $P(E)_{\mid \theta}$.

Se tivermos outro referencial admissível sobre $\theta, \tilde{s}: x \rightarrow P(E)_{x}$, procedendo exatamente da mesma forma que fizemos na construção de trivializações locais adaptadas a seções de fibrados principais, por $\phi^{3}$ ser um difeomorfismo, existe $g: \theta \rightarrow G$ suave tal que $\tilde{s}(x)=$ $g(x)(s(x))$, i.e.: $\phi^{-1}(\tilde{s}(x))=(x, g(x))$. Claramente $g^{-1}(x)=g(x)^{-1}$ que é suave $s(x)=$ $g(x)^{-1}(\tilde{s}(x))$ portanto

$$
\begin{aligned}
\phi^{\tilde{s}}: \theta \times G & \rightarrow P(E)_{\mid \theta} \\
(x, h) & \longmapsto h(\tilde{s}(x))
\end{aligned}
$$

\footnotetext{
${ }^{5}$ Omitindo o superíndice " $s$ ".
} 
induz estrutura difeomórfica àquela induzida por $\phi^{s}$ e portanto a estrutura diferenciável de $P(E)$ está bem definida, e portanto $P(E)$ é $G$-fibrado principal. Temos também que, como as as seções de $P(E)$ são dadas localmente por $\phi(\theta \times\{g\})$ para $g$ fixo, elas são simplesmente referenciais admissíveis, e $\phi^{s}$ é trivialização local adaptada a $s$.

Agora, dada uma conexão admissível $\nabla$ em $E$ (i.e.: tal que o transporte paralelo leva base admissível em base admissível), a maneira que pareceria óbvia de obter uma conexão em $P(E)$ seria aplicando essa conexão em cada campo de um referencial. Em outras palavras, aplicando o transporte paralelo infinitesimal em cada elemento de um referencial de $E$ (que equivale a uma seção de $P(E)$ ). Mais especificamente, dados um referencial $\left\{e_{i}\right\}_{i=1}^{k}$ sobre $\theta$ (que identificaremos com a seção $s: \theta \rightarrow P(E)$ ), um vetor $v \in T_{x} M$ tangente à curva $\sigma: I \rightarrow M$ em $t=0$, e chamando sugestivamente a forma de conexão correspondente a $\nabla$ nessa base de $\widetilde{\omega}^{s}$, obtemos, utilizando (1.106) e (1.66):

$$
\prod_{i=i}^{k} \frac{d}{d t}{ }_{\mid t=0}\left(P_{\sigma}(0, t) e_{i}(\sigma(t))\right)=: \frac{d}{d t}{ }_{\mid t=0}\left(P_{\sigma}(0, t) s(\sigma(t))\right)=\widetilde{\omega}^{s}(v) s(x)
$$

Pelo Teorema 15, como $s$ é referencial admissível, $\widetilde{\omega}^{s}(v) \in \mathfrak{g} \equiv \operatorname{End}_{\mathfrak{g}}\left(E_{x}\right)$, i.e.: temos uma representação $d \rho: \mathfrak{g} \rightarrow \operatorname{End}\left(E_{x}\right)$ tal que $\widetilde{\omega}^{s}(v)=d \rho\left(\omega^{s}(v)\right)$, onde $\omega^{s}(v) \in \mathfrak{g}$. Além disso, por (1.73) nós temos que dado outro referencial $\tilde{s}$, por $d \rho$ ser representação linear vale

$$
\int: G \rightarrow \operatorname{Aut}\left(E_{x}\right) \quad \omega^{\tilde{s}}=\operatorname{Ad}(g) \circ \omega^{s}+(d g) g^{-1}
$$

portanto, satisfizemos as hipóteses da Proposição 16, e podemos definir unicamente a forma de conexão $\omega=\mathrm{I}^{-1} \circ \widehat{V}$ no fibrado principal tal que tenhamos $s^{*} \omega=\omega^{s}$. A saber, por (2.26):

$$
\omega_{p}(w)=\omega_{p}\left(\left(d \phi^{s}\right)_{(x, e)}^{-1}(u, X)\right):=\omega_{x}^{s}(u)+X
$$

onde $x \in \theta, s$ é um referencial tal que a base sobre $x$ é dada por $s(x)=p, \phi^{s}$ é a trivialização local adapatada a $s$ destrinchada acima, $u \in T_{x} M, X \in \mathfrak{g}$ e $\omega^{s}$ é a forma de conexão em $E$ relativa a $\nabla$ e ao referencial $s$.

Por outro lado, se nos for dado $\omega \in \mathcal{C}(P(E))$, dado um referencial $s$ basta definirmos a forma de conexão em $E$ relativa a $s$ como uma representação de $\omega^{s}=s^{*} \omega$, i.e.: $\widetilde{\omega}^{s}=d \rho\left(s^{*} \omega\right)$. Utilizando o resultado do Teo.22 e do Teo.8, provamos então:

Theorem 24 Existe correspondência bijetora $\nabla \rightarrow H$ entre conexões em $E$ e em $P(E)$.

\section{Métricas Invariantes Revisitadas}

Seja $\pi: P \rightarrow M$ um $G$-fibrado principal, com $G$ ainda compacto. Seja $\langle\cdot, \cdot\rangle$ um produto interno Ad-invariante ${ }^{6} \mathrm{em} \mathfrak{g}$, i.e.: $\langle\operatorname{Ad}(g) u, \operatorname{Ad}(g) v\rangle=\langle u, v\rangle$ para quaisquer $u, v \in \mathfrak{g}$. Utilizando o isomorfismo dado por $\mathrm{I}_{p}: \mathfrak{g} \simeq \mathrm{V}_{p}$, por (2.1) nós temos uma métrica riemanniana $G$-invariante sobre o fibrado vertical. Isto é, sejam $\mathrm{I}_{p}(u), \mathrm{I}_{p}(v) \in \mathrm{V}_{p}$ então denotando da

\footnotetext{
${ }^{6}$ Como $G$ é compacto, admite métrica bi-invariante, se $G$ for conexo isto é equivalente a um produto interno Ad-invariante em g.
} 
mesma forma o produto interno em V definimos: $\left\langle\mathrm{I}_{p}(v), \mathrm{I}_{p}(u)\right\rangle_{p}:=\langle u, v\rangle$. Logo, para $g \in G$ utilizando (2.1)

$$
\begin{gathered}
\left\langle L_{g_{*}}\left(\mathrm{I}_{p}(v)\right), L_{g_{*}}\left(\mathrm{I}_{p}(u)\right)\right\rangle_{g \cdot p}=\left\langle\mathrm{I}_{g \cdot p}(\operatorname{Ad}(g) u), \mathrm{I}_{g \cdot p}(\operatorname{Ad}(g) v)\right\rangle_{g \cdot p} \\
=\langle\operatorname{Ad}(g) u, \operatorname{Ad}(g) v\rangle=\langle u, v\rangle
\end{gathered}
$$

Chamaremos uma métrica $\gamma$ de invariante para $P$ se $\gamma$ for uma métrica riemanniana em $P$ que é invariante em relação à ação de $G$ e se restringe à métrica definida acima sobre o fibrado vertical.

Agora se $\mathrm{V}^{\perp}$ for o subfibrado de $T P$ ortogonal a $\mathrm{V}$ em relação a $\gamma$, então claramente $\mathrm{V}^{\perp}$ é transversal a V. Como V e $\gamma$ são $G$-invariantes, também o será $V^{\perp}$, portanto $\mathrm{V}^{\perp}=\mathrm{H}$ é uma conexão para $P$. Além disso como vimos, há um isomorfimo entre $\mathrm{H}_{p}$ e $T_{\pi(p)} M$, e portanto induzimos uma única métrica sobre $M$ pela projeção $\pi$. Analogamente se tivermos uma métrica $h$ em $M$ e um fibrado horizontal qualquer $\mathrm{H}$, temos uma única métrica induzida em $\mathrm{H}$ por $\pi^{*} h$. Portanto como $T P=\mathrm{V} \oplus \mathrm{H}$ e a restrição da métrica para $\mathrm{V}$ é canônica, temos uma única métrica $\gamma$ em $P$, tal que $\mathrm{H} \perp \mathrm{V}$, definida por uma conexão $\mathrm{H}$, uma métrica $h$ em $M$ e uma métrica de $\mathfrak{g}$ Ad-invariante $\alpha$. A saber, lembrando que dado $\mathrm{H}$, há uma única forma de conexão correspondente $\omega_{p}=\mathrm{I}_{p}^{-1} \circ \widehat{V}$, temos

$$
\gamma=\pi^{*} h+\alpha \circ \omega
$$

Se $u, v \in \mathrm{V}_{\boldsymbol{p}}$ então

$$
\gamma_{p}(u, v)=\alpha\left(\mathrm{I}_{p}^{-1}(u), \mathrm{I}_{p}^{-1}(u)\right)
$$

já que $d \pi_{p}(v)=d \pi_{p}(u)=0$. Se $u, v \in \mathrm{H}_{p}$ temos

$$
\gamma_{p}(u, v)=\gamma_{\pi(p)}\left(d \pi_{p}(u), d \pi_{p}(v)\right)
$$

já que $\omega_{p}(u)=\omega_{p}(v)=0$ e finalmente se $u \in \mathrm{H}_{p}$ e $v \in \mathrm{V}_{p}$ então $\gamma_{p}(u, v)=0$ já que $\omega_{p}(u)=0$ e $d \pi_{p}(v)=0$. Como tanto $\pi^{*} h$ quanto $\alpha \circ \omega$ são $G$-invariantes, $\gamma$ é $G$-invariante. Chamamos tais métricas de métricas de fibrado (bundle metrics).

Como veremos mais tarde, existem relações interessantes entre a geometria de $(M, h)$ e a de $(P, \gamma)$ envolvendo a conexão. Estas relações são centrais à unificação da gravitação e campos de Yang-Mills.

\subsection{Fibrados Associados}

Dado um $G$-fibrado vetorial $E$ sobre $M$, com fibra típica isomorfa ao espaço vetorial $\mathbb{R}^{k}$, na Sec.2.3 nós construímos um $G$-fibrado principal sobre $M$ correspondente. O que podemos dizer sobre o procedimento inverso, i.e.: dado um $G$-fibrado principal sobre $M$ podemos associar a ele um fibrado vetorial com fibra típica isomorfa a $\mathbb{R}^{k}$ e variedade base $M$ ? Na verdade veremos que podemos atingir um resultado mais geral, com a fibra típica sendo difeomorfa a uma variedade suave qualquer onde o grupo age como grupo de transformações. Para ilustrar bem nosso objetivo, tomemos o fibrado dos referenciais $P(E)$, a partir do qual tentaremos reconstruir $E$. A idéia que surge naturalmente é tomar um vetor em $E_{x}$ como uma escolha de valores para os elementos de uma base em $p \in P(E)$, ou seja, 
como suas coordenadas $\left(a^{1}, \cdots, a^{k}\right)$ na base $p$. Suponhamos que $x \in \theta \subset M, v \in E_{x}$ e $p=\left(e_{1}(x), \cdots, e_{n}(x)\right)$, onde os $e_{i}$ são seções linearmente independentes de $\left.E\right|_{\theta}$. Então $v=a^{1} e_{1}(x)+\cdots+a^{k} e_{k}(x)$ é a ação natural de $P$ em $\mathbb{R}^{k}$. Chamando $\left(a^{1}, \cdots, a^{k}\right):=\tilde{v}$, esta ação é simplesmente dada por $\tilde{v} p$, a multiplicação de uma matriz linha por uma matriz coluna, e poderíamos pensar em identificar dessa forma, $v \simeq(\tilde{v}, p) \in \mathbb{R}^{k} \times P$. Obviamente essa identificação é insuficiente, pois há muitas bases e muitos elementos de $\mathbb{R}^{k}$ correspondentes que resultariam no vetor de $E_{x}$ em questão. Precisamos tomar o quociente pelos isomorfismos lineares das bases, i.e.: pela ação dos elementos da fibra $\pi^{-1}(x)$. Agora, identificando a matriz linha $\tilde{v}$ a uma 1-forma em $E_{x}^{*}$, nós temos que por um isomorfismo $g(x)$ de $E_{x}$, como vimos no primeiro capítulo, temos as seguintes transformações:

$$
\begin{aligned}
& g(x): \tilde{v} \quad \longmapsto \tilde{v} g(x)^{-1} \\
& e_{i}(x) \longmapsto g(x)\left(e_{i}(x)\right)
\end{aligned}
$$

Claramente temos então que $\tilde{v} g(x)^{-1} g(x)(p)=\tilde{v} p=v$ e portanto também identificaríamos $v \simeq\left(\tilde{v} g(x)^{-1}, g(x)\left(e_{i}(x)\right)\right.$. A solução para eliminarmos esta redundância é óbvia, quocientarmos pela relação de equivalência em $P(E) \times \mathbb{R}^{k}$ dada por $\left(\rho(g) p, v \rho\left(g^{-1}\right)\right) \sim(p, v)$, ou, substituindo $\rho(g)$ por $g$, onde $\rho: G \rightarrow \operatorname{Aut}(E)$, podemos escrever mais sucintamente:

$$
\left(g p, v g^{-1}\right) \sim(p, v)
$$

o que é compatível com nosso uso de multiplicação à esquerda pelo grupo (a representação do grupo em $\mathbb{R}^{k}$ vai à direita pela inversa, já que $\mathbb{R}^{k}$ corresponde ao espaço dual). A notação usual é feita para um fibrado $G$-principal $P$, no qual $G$ age à direita, e nos conformaremós a ela. Um breve adendo: se $G$ age à direita sobre dois espaços, $A, B$ e existe uma ação de $\mu: A \times B \rightarrow C$, para um outro espaço qualquer $C$, e se essa ação é invariante pela ação do mesmo elemento de $G$ nos dois espaços, i.e.: $\mu\left(a \cdot{ }_{A} g, b \cdot_{B} g\right)=\mu(a, b)$ dizemos que ela é $G$-equivariante.

No nosso caso, a representação do grupo é a mesma em $E_{x} \simeq \mathbb{R}^{k}$ e $P\left(E_{x}\right)$, portanto o quociente pela ação do grupo como acima está inclusa na ação da base sobre as coordenadas, i.e.: $[p, v]=p v$. No caso geral, a ação do grupo pode ser distinta para os dois espaços, por isso definimos:

Definition 20 Seja $(P, \pi, M)$ um G-fibrado principal sobre o qual G age a direita por".", $e$ seja $F$ um espaço no qual $G$ age à esquerda (cuja ação denotaremos por “"), então definimos $o$ fibrado associado a $P$ pela ação de $G$ em $F$ como o fibrado

$$
P_{F}=P \times_{G} F:=P \times F / \sim
$$

onde definimos a relação de equivalência, juntamente com a ação do grupo em $P \times F$ (que também denotaremos por " "), como

$$
(p, v) \sim\left(p \cdot g, g^{-1} \cdot v\right):=(p, v) \cdot g
$$

A projeção é dada por

$$
\begin{aligned}
\pi_{F}: & P_{F} \longrightarrow M \\
& {[p, v] \mapsto \pi(p) }
\end{aligned}
$$


Notemos que que $\pi_{F}$ está bem definida, já que se $\left(p_{1}, v_{1}\right) \sim\left(p_{2}, v_{2}\right)$ então $p_{2}=p_{1} \cdot g$ para algum $g \in G$ e portanto $\pi\left(p_{2}\right)=\pi\left(p_{1}\right)$. Precisamos agora voltar e mostrar que a motivação do conceito de fibrado associado faz sentido, i.e.: que cada fibra de $P_{F}$ é difeomorfa a $F$.

Theorem 25 Para cada $x \in M$, a fibra $\pi_{F}^{-1}(x)$ é difeomorfa a $F$.

Dem: Fixado um ponto $p \in P$ sobre $x \in M$ existe um mapa associado $\imath_{p}: F \rightarrow P_{F}$ definido por $\imath_{p}(v):=[p, v]$, no nosso exemplo esse mapa corresponde a tomarmos $k$ coordenadas e associar-las à base $p$. Agora, $\pi_{F}[p, v]=\pi(p)=x \operatorname{logo} \imath_{p}(F) \subset \pi_{F}^{-1}(x)$. Agora, para $[q, u] \in \pi_{F}^{-1}(x)$, definimos

$$
\begin{aligned}
\jmath_{p}: & \pi_{F}^{-1}(x) \rightarrow F \\
& {[q, v] \longmapsto g_{p}(q) \cdot v }
\end{aligned}
$$

onde $g_{p}: \pi^{-1}(x) \rightarrow G$ é o mapa que fornece o elemento em $G$ que "liga" $p$ a $q$, i.e: $p \cdot g_{p}(q)=q$, como mostramos nas primeiras seções deste capiítulo, é um difeomorfismo. Provemos que o mapa $\jmath_{p}$ está bem definido: para $g \in G$, nós temos $g_{p}(q \cdot g)=g_{p}(q) g$ já que, utilizando a definição de $g_{p}$ :

$$
p \cdot\left(g_{p}(q \cdot g)\right)=q \cdot g=\left(p \cdot g_{p}(q)\right) \cdot g=p \cdot\left(g_{p}(q) g\right)
$$

Logo

$$
J_{p}\left[q \cdot g, g^{-1} \cdot v\right]=g_{p}(q) g g^{-1} \cdot v=g_{p}(q) \cdot v
$$

Então obtemos, qualquer que seja $v \in F$,

$$
\begin{aligned}
& \text { (1) } \imath_{p} \circ \jmath_{p}[q, v]=\imath_{p}\left(g_{p}(q) \cdot v\right)=\left[p, g_{p}(q) \cdot v\right]=\left[p \cdot g_{p}(q), v\right]=[q, v] \\
& \text { (11) } \jmath_{p} \circ \imath_{p}(v)=\jmath_{p}[p, v]=g_{p}(p) \cdot v=v
\end{aligned}
$$

e portanto $\imath_{p}$ e $\jmath_{p}$ são inversas suaves.

Nós temos ainda que se $\theta$ é um aberto de $M$,

$$
\pi_{F}^{-1}(\theta) \simeq\left(\pi^{-1}(\theta) \times F\right) / G \simeq(\theta \times G \times F) / G \simeq \theta \times F
$$

Portanto é localmente trivial, logo podemos introduzir em $P_{F}$ uma estrutura diferenciável requerendo que $\pi_{F}^{-1}(\theta)$ seja uma subvariedade aberta de $E$ difeomorfa a $\theta \times F$ pelo difeomorfismo induzido por uma trivialização qualquer $\pi^{-1}(\theta) \simeq \theta \times G$. Dadas duas tais trivializações $\psi_{1}, \psi_{2}$ sobre $\theta$, i.e.:

$$
\begin{aligned}
\psi_{i}: \theta \times F & \rightarrow \pi_{F}^{-1}(\theta) \\
(x, v) & \longmapsto\left[s_{i}(x), v\right]
\end{aligned}
$$

onde $s_{i}: \theta \rightarrow \pi^{-1}(\theta)$ é seção suave de $P$ nós obtemos ${ }^{7}$, utilizando o mapa de transição $g_{12}: \theta \rightarrow G$ entre as seções $s_{1}, s_{2}$, que

$\psi_{2}^{-1} \circ \psi_{1}(x, v)=\psi_{2}^{-1}\left[s_{1}(x), v\right]=\psi_{2}^{-1}\left[s_{1}(x) \cdot g_{12}(x), g_{12}^{-1} v\right]=\left[s_{2}(x), g_{12}^{-1}(x) \cdot v\right]=\left(x, g_{12}^{-1}(x) \cdot v\right)$

Nós obtemos então que se começamos com um $E$ é um $G$-fibrado vetorial com fibra típica $V$, e tomamos $G \equiv \operatorname{Aut}_{G}(V)$, então $P(E) \times_{G} V=E$.

\footnotetext{
${ }^{7}$ Note que utilizamos a mesma construção para obtermos a correspondência entre diferentes trivializações quaisquer no fibrado principal.
} 
Agora, nós já provamos que existe correspondência bijetora entre conexões em $E$ e conexões em $P(E)$ (Teo. 24), simplesmente definindo a forma de conexão em $\pi_{V}^{-1}(\theta)$ como $\widetilde{\omega}_{x}^{s}=d \rho_{x}\left(s^{*} \omega\right)_{x}$ onde $\omega_{p}: T_{p} P \rightarrow \mathfrak{g}, d \rho_{x}: \mathfrak{g} \rightarrow \operatorname{End}_{\mathfrak{g}}\left(E_{\pi(p)}\right)$ é uma representação de $\mathfrak{g}, s$ é uma seção de $P$ sobre $\theta$ e $\omega$ é uma forma de conexão em $P$.

Se tomarmos a descrição por transporte paralelo, isto é equivalente a, dado um ponto $p \in P(E)$ sobre $x \in M$, uma curva suave $\alpha: I \rightarrow M$ tal que $\alpha(0)=x$ e um vetor de $E_{x}$, $v=[p, \tilde{v}]$ onde $\tilde{v}$ é a projeção de $v$ na base $p$, definirmos

$$
P_{\alpha}(0, t) v:=\left[\widetilde{\alpha}_{p}(t), v\right]=\widetilde{\alpha}_{p}(t) v
$$

onde $\widetilde{\alpha}_{p}(t)$ é o único levantamento horizontal de $\alpha(t)$ em $P$ passando por $p$ em $t=0$, ou seja, mantemos as coordenadas do vetor fixo em termos de uma dada base paralela ao longo de $\alpha(t)$. Em outras palavras, derivamos como funções em $\mathbb{R}^{k}$ as coordenadas de um campo (seção de $E$ ) em relação a uma base paralela. Como mostramos que temos o conceito de levantamento horizontal de curvas em fibrados principais gerais, podemos tomar essa definição para o caso geral de fibrado associado.

Relembrando, nós temos, como $\jmath_{p}: F \rightarrow F_{\pi(p)}$ é difeomorfismo, que para ćada $p \in \pi^{-1}(x)$ e $q \in \pi_{F}^{-1}(x)$ existe um único $v(q, p) \in F$ tal que $\left(p, v(q, p) \in q\right.$. Seja então $s: M \rightarrow P \times_{G} F$ uma seção, para todo $p \in P$ existe $v(s(\pi(p)), p)=: \varphi^{s}(p)$ tal que $\left[p, \varphi^{s}(p)\right]=s(\pi(p))$, onde denotamos a função que, dada essa seção, leva $p$ em $v(p)$ por $\varphi^{s}: P \rightarrow F$. Nós temos que $\varphi(p \cdot g)=g^{-1} \cdot \varphi^{s}(p)$ já que temos, para uma dada seção $s$ e dados $x \in \theta, p, q \in \pi^{-1}(x)$ :

$$
\left[q, \varphi^{s}(q)\right]=\left[p \cdot g, \varphi^{s}(p \cdot g)\right]=s(x)=\left[p, \varphi^{s}(p)\right]=\left[p \cdot g, g^{-1} \cdot \varphi^{s}(p)\right]
$$

Na nossa analogia do fibrado das bases, isso nos dá para cada campo $Y \in \Gamma(E)$ uma associação entre as bases sobre $\theta$ e a descrição de $Y$ sobre essas bases. O que nos resta agora para acharmos $\nabla_{X}^{P_{F}} Y$, onde $\nabla^{P_{F}}$ é a conexão em $P \times_{G} F$ e $X \in \Gamma(T M)$, é tomarmos, na analogia do fibrado das bases, a derivada dos coeficientes sobre uma base paralela ao longo de $X$. Como o levantamento horizontal está bem definido, é exatamente isso que fazemos:

$$
\varphi^{\nabla_{X}^{P_{F}}}=\tilde{X}\left[\varphi^{s}\right]
$$

onde $\tilde{X}$ é o levantamento horizontal de $X$, como descrevemos na seção anterior, e aqui o colchete designa derivação, e não quociente. Ambos os lados pertencem a $C^{\infty}(P, F)$ e o que obtemos é que, para todo $p \in P$,

$$
\left[p, \tilde{X}\left[\varphi^{s}\right](p)\right]=\nabla_{X}^{P_{F}} s(\pi(p))
$$

Lembrando que $R_{g_{*}} \widetilde{X}=\widetilde{X}$, e portanto a equivariância da ação de $G$ é mantida e vemos que a equação está bem-definida. Explicitamente, nós utilizamos a seguinte proposição:

Proposition 18 Seja $X$ um campo $C^{\infty}$ em $M$, e $F: M \rightarrow M$ um difeomorfismo, e seja $\theta(t, p)$ o fluxo de $X$, então $X$ é invariante por $F$ se e somente se $F(\theta(t, p))=\theta(t, F(p))$

Dem: Se $F(\theta(t, p))=\theta(t, F(p))$ então nós temos que

$$
\left.d F_{p}\left(\left.\frac{d}{d t}\right|_{t=o} \theta(t, p)\right)\right)=d F_{p}\left(X_{p}\right)
$$


Mas

$$
\left.\frac{d}{d t}\right|_{t=o} \theta(t, F(p))=X_{F(p)}
$$

Da mesma forma

$$
\frac{d}{d t}_{\left.\right|_{t=o}} F(\theta(t, p))=d F_{p}\left(X_{p}\right)=X_{F(p)}=\frac{d}{d t}_{\left.\right|_{t=o}}(\theta(t, F(p)))
$$

portanto, pela unicidade de curvas integrais:

$$
\theta(t, F(p))=F(\theta(t, F(p)))
$$

Logo temos, chamando ainda $\theta(t, p)$ o fluxo de $X$ por $p$,

$$
\begin{aligned}
\varphi^{\nabla_{X}^{P_{F}}(g \cdot p)} & =\tilde{X}\left[\varphi^{s}\right](g \cdot p) \\
& =\left.\frac{d}{d t}\right|_{t=o}\left(\varphi^{s}(\theta(t, g \cdot p))\right) \\
& =\left.\frac{d}{d t}\right|_{t=o} \varphi^{s}(g \cdot(\theta(t, p))) \\
& =g^{-1} \cdot \varphi^{\nabla_{X}^{P_{F}}}(p)
\end{aligned}
$$

Outra observação importante é a de que, olhando bem para o lado esquerdo de (2.50), percebemos que na linguagem de fibrado das bases, ele representa a derivada dos coeficientes de um campo ao longo de uma curva horizontal de bases passando por $p$, justamente o que procurávamos. Notamos também que a conexão em $P_{F}$ "desceu" de uma $G$-conexão de $P$, já que utilizamos tanto levantamento horizontal quanto $G$-invariância.

Suponhamos agora que $V$ seja um espaço vetorial riemanniano, com métrica $\langle\cdot, \cdot\rangle$ sobre o qual age a representação $\rho: G \rightarrow V$. Nós podemos inicialmente tentar induzir uma métrica em $P_{V}=P \times_{G} V$ definindo, para qualquer $p \in \pi^{-1}(x)$ e $v, v^{\prime} \in V$ :

$$
\left\langle[p, v],\left[p, v^{\prime}\right]\right\rangle_{\pi_{V}^{-1}(x)}:=\left\langle v, v^{\prime}\right\rangle
$$

Lembramos que qualquer elemento de $\pi_{V}^{-1}(x)$ pode ser escrito em termos de qualquer $p \in$ $\pi^{-1}(x)$. Mas para que este produto interno faça sentido precisamos tomar o mesmo $p$ (o que equivaleria, no fibrado das base, a tomar o produto interno das coordenadas escritas na mesma base). Verifiquemos se isto está bem definido, i.e.: se o produto interno não depende do ponto $p$ que escolhemos:

$$
\left\langle[p, v],\left[p, v^{\prime}\right]\right\rangle_{\pi_{V}^{-1}(x)}=\left\langle\left[p \cdot g, \rho\left(g^{-1}\right) v\right],\left[p \cdot g, \rho\left(g^{-1}\right) v\right]\right\rangle_{\pi_{V}^{-1}(x)}=\left\langle\rho\left(g^{-1}\right) v, \rho\left(g^{-1}\right) v^{\prime}\right\rangle
$$

Então nós temos que só podemos passar a métrica consistentemente para o fibrado associado se a ação do grupo em $V$ for ortogonal em relação a métrica, i.e.: $\rho: G \rightarrow \mathbf{O}(V)$. Isso implica não só que a métrica induzida em $P_{F}$ é $G$-invariante, mas que é também compatível com qualquer $G$-conexão provinda de $P$. Vejamos como: denotando também por $\rho_{x}$ a ação de $G$ em $\pi_{F}^{-1}(x)$, temos que $\rho_{x}: G \rightarrow \mathbf{O}\left(\pi_{F}^{-1}(x)\right)$. Logo

$$
d\left(\rho_{x}\right)_{e}: \mathfrak{g} \rightarrow \mathfrak{s o}\left(\pi_{F}^{-1}(x)\right)
$$

o que implica que a representação de $\omega^{s}$ será uma matriz anti-simétrica, o que em seu turno implica que a conexão é compatível com a métrica. 


\section{Chapter 3}

\section{Campos de Yang-Mills e Classes Características}

Há uma máscara de teoria sobre toda a face da natureza. - William Whewell

Como pode ser que a matemática, sendo antes de tudo um produto do pensamento humano independente da experiência, é tão admiravelmente adaptada aos objetos da realidade? - Albert Einstein

\subsection{Yang-Mills}

\section{Introdução}

A aplicabilidade da teoria da matemática é um assunto filosoficamente interessante e profundo; será que a matemática é tão útil para a descrição da Natureza porque a seleção natural de Darwin beneficiou um processamento de informações adaptado à realidade, o que conhecemos por lógica? Ou simplesmente é o que temos em mão e procuramos a relação inversa; adaptar a realidade à matemática? A teoria de Gauge e a equação de Yang-Mills constituem exemplos dos mais formidáveis de uma convergência não intencional de física e matemática, reforçando a primeira hipótese. É bem verdade que Yang e Mills procuravam exclusivamente uma generalização das equações de Maxwell, sem nenhum conhecimento de sua relação com uma interpretação geométrica por fibrados (interpretação que procuramos esmiuçar).

Sua busca era mais que justificada: a teoria da eletrodinâmica quântica é uma das mais bem sucedidas da história da física. O objetivo da física (ou o de uma grande parte dos físicos) era (e talvez ainda seja) o de colocar todas as partículas no mesmo pé que o fóton. Apesar de não tratarmos aqui do aspecto quântico das teorias de Gauge, segundo Atiyah 1 "podemos dizer que uma compreensão profunda da teoria clássica é provavelmente um pré-requisito para o desenvolvimento da teoria quântica".

Faremos, antes de começarmos a exposição matemática mais pesada, uma breve introdução física dos conceitos da teoria clássica de Gauge, muitos deles já explorados por nós nos capítulos anteriores.

\footnotetext{
${ }^{1}$ Ver [11] que seguimos livremente nesta introdução.
} 
Imaginemos uma partícula em $M$, variedade semi-riemanniana quadridimensional. Suponhamos que essa partícula tenha alguma espécie de estrutura interna i.e.: ela tem uma posição $x \in M$ e está em um estado interno particular neste ponto. Suponhamos ainda que este espaço interno possua simetrias suaves, modeladas pelo grupo de Lie $G$. Consideraremos então o espaço total de todos os estados de uma tal partícula, que chamaremos de $E$.

A curvatura $\Omega$ pode ser tomada como a distorção das fibras provocada pelo campo ${ }^{2}$, se pensarmos no campo como dado por seus efeitos locais. Podemos identificar coerentemente nossos espaços internos sobre $M$ se para quaisquer caminhos que tomarmos entre dois pontos o estado interno final da partícula for o mesmo. Se assumirmos que o estado interno da partícula é levado ao longo do trajeto por transporte paralelo, i.e.: de modo que "conserve" seu estado interno, pelo Teo.14 essa condição é equivalente ${ }^{3}$ a não termos curvatura, ou campo externo, já que não há maneira de medi-lo por seu efeito se todos os caminhos entre dois pontos não induzem nenhuma diferença na estrutura interna da partícula.

Qualquer identificação de espaços internos é chamada de uma escolha de Gauge, e uma mudança de um gauge a outro é chamado de transformação de gauge, que a cada ponto $x$ associa uma transformação do espaço interno $G$. Sem curvatura, todas são equivalentes, e se ligarmos um campo externo todas detectarão igualmente discrepâncias no estado interno final de partículas tomando caminhos distintos.

Essa identificação de campos com distorções geométricas também é central à teoria da relatividade geral. A diferença aqui é que essa distorção não ocorre no espaço-tempo, mas na geometria de um espaço de estrutura interna, superposto ao espaço-tempo. Como assumimos que o grupo de estados internos é bem mais simples do que o de transformações de coordenadas (tem dimensão finita), isto se traduz em uma relativa simplificação da teoria em comparação com a geometria riemanniana da relatividade geral.

Historicamente, potenciais foram introduzidos como um instrumento de simplificação das equações do campo, e a ambiguidade inerente em sua escolha (liberdade de Gauge) era tida como uma indicação de que não possuía significado físico. Pelo ponto de vista geométrico, o potencial, identificado à conexão, têm existência própria e bem definida, somente a escolha de um gauge (ou referencial) não tem significado físico. Ou seja uma conexão nos fornece um meio de quantificar a variação de estados internos ao longo de trajetórias em $M$.

Explicitamente, tomamos $P$ como o $G$-fibrado principal das bases $G$-admissíveis sobre $M$ (ver Sec2.3), por exemplo os eixos de isospin sobre $M$. A conexão $\omega$ nos fornece uma identificação intrínseca entre (bases de) estados internos sobre diferentes pontos, ela nos diz como manter uma base de estados internos (e.g.: de isospin) "fixa" ao longo de uma trajetória qualquer $\gamma$. Em termos dessa base é fácil quantificar a variação do estado interno da partícula (de seu isospin) ao longo de $\gamma$ : simplesmente utilizamos a conexão em $E$ como fibrado associado a $P$, explicitada ${ }^{4}$ em (2.50).

Até aqui consideramos uma partícula como no caso clássico, como tendo trajetória bem definida etc. Apesar da abordagem ortodoxa da mecânica quântica ser feita através de campos, essa nossa descrição pode ser associada a formulação por integrais de trajetória de

\footnotetext{
${ }^{2} \mathrm{Na}$ área cercada por um paralelogramo infinitesimal, dado por duas direções em $x$.

${ }^{3}$ É equivalente em um espaço simplesmente conexo, não o sendo temos o efeito intrigante de AharonovBohm [8].

${ }^{4}$ I.e.: simplesmente derivamos as coordenadas da decomposição do estado interno em relação a uma base "fixa" ao longo de $\gamma$.
} 
Feynman, equivalente à formulação usual.

Mais explicita e formalmente, a exemplo de [10], mencionamos e comentamos os ingredientes básicos para a descrição clássica da interação de uma partícula com um campo de gauge:

1. Uma variedade suave (semi) riemannana $M$. - Este é simplesmente o espaço onde as partículas vivem.

2. Um espaço vetorial de dimensão finita $F$ equipado com um produto interno $\langle\cdot, \cdot\rangle$. $\quad$ - Na interpretação ortodoxa, este é o espaço onde as funções de onda das partículas tomam seus valores. Este espaço é determinado pela estrutura interna da partícula (fase, isospin, etc) e é chamado de espaço interno. Exemplos típicos são $\mathbb{C}, \mathbb{C}^{2}, \mathbb{C}^{4}$ ou as álgebras de Lie $\mathfrak{u}(1), \mathfrak{s u}(2)$. Pelo produto interno se computa a norma de funções de onda e portanto probablidades quânticas.

3. Um grupo de Lie $G$ e uma representação $\rho: G \rightarrow \mathbf{G L}(F)$ ortogonal em relação a $\langle\cdot, \cdot\rangle$. $\quad-G$ age então sobre as bases dos estados internos sobre cada ponto. Como vimos em (2.51), a ortogonalidade da representação é necessária para que o produto interno não dependa da base de estados internos que escolhemos. Como vimos em (Sec.1.6), existe bijeção entre $G$ e as bases ortonormais $G$ admissíveis sobre um dado ponto $x \in M$.

4. Um $G$-fibrado principal sobre $M:(P, \pi, M, G)$. - Pela Sec.2.3 este fibrado pode ser identificado ao fibrado das bases $G$ admissíveis sobre $M$. A fibra sobre cada ponto é uma cópia de $G$ vista como todas as bases ortonormais $G$-admissíveis dos estados internos. Uma seção de $P$ é um referencial $G$-admissível relativo ao qual descrevemos nossa função de onda.

5. Uma conexão $\omega$ em $P$, com curvatura $\Omega$. - Essa conexão nos fornece a variação intrínseca das bases. Aplicada sobre um referencial local $s$, nós obtemos o potencial de gauge local, $\mathcal{A}=s^{*} \omega$. Similarmente obtemos o campo local de gauge $\mathcal{F}=s^{*} \Omega$.

6. Uma seção global $\Phi$ do fibrado vetorial associado $P \times_{\rho} F$ - Campos de matéria serão associados a tais seções que satisfaçam as equações de Euler-Lagrange de algum funcional de ação que envolva os potenciais locais $\mathcal{A}$. Como vimos em Sec.2.4, dado um referencial podemos associar localmente estas seções a funções $G$-equivariantes $\psi: \theta \rightarrow F$, que nos fornecerão as chamadas funções de onda.

7. Uma ação $S(\Phi, \omega)$ cujos pontos estacionários são as soluções clássicas. Tipicamente este funcional é da seguinte forma:

$$
S(\Phi, \omega)=c \int_{M}\|\Omega\|^{2}+c_{1}\|D \Phi\|^{2}
$$

Onde $D$ é a derivada exterior covariante determinada por $\omega$ Teo.26. A constante $c$ é chamada de constante de normalização e $c_{1}$ é a constante de acoplamento. Discutiremos as normas utilizadas na seção seguinte. Não trataremos do caso dos campos com matéria, ou seja, faremos $c_{1}=0$. Estamos interessados somente na dinâmica dos campos. 
Ou seja, todo o trabalho que tivemos até aqui foi o de apresentar boa parte do arcabouço teórico de uma teoria física baseada nestas suposições. O que nós queremos agora, é, assim como na teoria da relatividade geral, minimizar um funcional escalar da curvatura, para que possamos achar uma configuração de campos que represente uma solução clássica do sistema. Para isso, ainda precisamos colocar alguns detalhes na teoria, como achar uma métrica bem definida no espaço de estados internos.

\section{Preliminares}

$\mathrm{Na}$ teoria de Yang-Mills, como vimos, os campos físicos de interesse são a curvatura e a conexão, que no caso de fibrados vetoriais, são representações da forma de conexão $\omega^{s}$ do fibrado principal. Como uma representação de $G$ em um dado $x \in M$, é uma aplicação $\rho_{x}: G \rightarrow \operatorname{Aut}\left(E_{x}\right)$, onde $E$ é o $G$-fibrado vetorial em questão; a representação correspondente da álgebra de Lie é $d\left(\rho_{x}\right)_{e}: \mathfrak{g} \rightarrow \operatorname{End}\left(E_{x}\right)$, ou seja, são formas a valores em representações da àlgebra de Lie $\mathfrak{g}$ sobre os endomorfismos de cada fibra vetorial, $\operatorname{End}\left(E_{x}\right)$. Estamos interessados em estudar seções de formas de conexão em $E$, e portanto precisaremos considerar o fibrado $\operatorname{End}(E) \simeq E \otimes E^{*}$. Podemos proseguir de duas maneiras equivalentes: tomando diretamente o fibrado vetorial $\operatorname{End}(E)$ como o protuto tensorial de fibrados, ou pelo intermédio do fibrado associado $P_{F}:=(P \times F) / G$ onde $G$ age pela conjugação no espaço vetorial $F=\operatorname{End}(V)$, tentaremos oferecer uma comparação entre os dois. Para que possamos medir de alguma maneira a "intensidade" de formas de conexão sobre um dado ponto da base, precisamos de alguma noção de produto interno, que, pelo que vimos na seção anterior, seja invariante pela ação do grupo em $F$ (2.52).

Podemos induzir um mapa linear natural

$$
\begin{aligned}
\operatorname{tr}: \operatorname{End}(V) & \longrightarrow \mathbb{R} \\
v \otimes \lambda & \longrightarrow \lambda(v)
\end{aligned}
$$

Pra vermos que esse mapa coincide com o que usualmente chamamos de traço, escolhemos bases duais $\left\{e_{i}\right\}$ e $\left\{e^{i}\right\}$ de $V$ e de $V^{*}$ respectivamente, escrevendo $T \in \operatorname{End}(V)$ nessa base temos

$$
\operatorname{tr}(T)=T_{j}^{i} e^{j}\left(e_{i}\right)=T_{j}^{j}
$$

Claramente, se $T=v_{1} \otimes \lambda^{1}, S=v_{2} \otimes \lambda^{2} \in \operatorname{End}(V)$, (lembramos que elementos dessa forma geram $\operatorname{End}(V))$ temos

$$
\operatorname{tr}(T S)=\operatorname{tr}\left(v_{1}\left(\lambda^{1}\left(v_{2}\right)\right) \otimes \lambda^{2}\right)=\lambda^{1}\left(v_{2}\right) \operatorname{tr}\left(v_{1} \otimes \lambda^{2}\right)=\lambda^{1}\left(v_{2}\right) \lambda^{2}\left(v_{1}\right)=\operatorname{tr}(S T)
$$

Definimos o negativo da forma de Killing:

$$
\begin{aligned}
& K: \mathfrak{g} \times \mathfrak{g} \rightarrow \mathbb{R} \\
& (h, j) \mapsto-\operatorname{tr}\left(\operatorname{ad}_{h} \circ \operatorname{ad}_{j}\right)
\end{aligned}
$$

que é assim claramente bilinear e simétrica. É possível ainda mostrar que a forma de Killing á invariante pela representação adjunta do grupo na álgebra e que, se $G$ for compacto, então $K$ é positivo definido se e somente se $G$ é semi-simples [16]. 
Lemma 2 Se g é a álgebra de Lie de um grupo compacto semi-simples, então a forma de Killing é positiva-definida em $\mathfrak{g}$, i.e.: para todo $X \neq 0, K(X, X)>0$.

Usualmente essa é a métrica para $\mathfrak{g}$, mas não a utilizaremos explicitamente, apenas como artifício para induzir uma métrica não -degenerada no fibrado associado e com isso um operador de Hodge. No caso de $\mathfrak{u}(n)$, como mostraremos a seguir, é possível utilizar o operador traço, que é o que faremos, pois, além da simplicidade formal, fica mais direta a conexão com classes de Chern e topologia de fibrados, que apresentamos no capítulo seguinte.

Agora, para $G$ compacto, é um fato que a álgebra de Lie de $G$ admite a seguinte decomposição

$$
\mathfrak{g}=\mathfrak{z} \oplus[\mathfrak{g}, \mathfrak{g}]
$$

onde $\mathfrak{z}=\operatorname{Ker}(\mathrm{ad})$ é o centro da álgebra, $\left.\log \mathrm{K}\right|_{\mathfrak{z}} \equiv 0$ e a parte $[\mathfrak{g}, \mathfrak{g}]$ é semi-simples. Logo, utilizando a métrica produto natural (ortogonal) em $\mathfrak{g}=\mathfrak{z} \oplus[\mathfrak{g}, \mathfrak{g}]$ temos que um outro produto interno em $\mathfrak{g}$ que seja positivo definido sobre o centro e proporcional a $K$ na parte semi-simples será positivo definido em sua totalidade. No caso de maior interesse para a física:

$$
\mathfrak{u}(n)=\mathfrak{u}(1) \oplus \mathfrak{s u}(n)
$$

Nesse caso, o centro são os múltilpos da matriz identidade e portanto é não degenerada nessa parte. Agora mostremos o traço e a froma de Killing são de fato proporcionais na parte semi-simples.

Assumindo que um grupo $G$ é simples se e somente se ele admite representação adjunta irredutível, suponhamos que temos duas formas bilineares invariantes por uma representação $\rho: G \rightarrow \operatorname{Aut}(V)$ em um espaço vetorial $V,\langle\cdot, \cdot\rangle_{1}$ e $\langle\cdot, \cdot\rangle_{2}$. Afirmamos que $B_{1}=\langle\cdot, \cdot\rangle_{1}$ e $B_{2}=\langle\cdot, \cdot\rangle_{2}$ são proporcionais. Para provar que isto de fato se dá, consideremos a aplicação $B_{1}: V \rightarrow V^{*}$. Como o núcleo desta aplicação é invariante pela ação de $\rho$, que é representação irredutível de $G$ em $\operatorname{Aut}(V)$, nós temos que ou $\operatorname{Ker} B_{1}=0$ ou $\operatorname{Ker} B_{1}=V$. Portanto podemos assumir sem perda de generalidade que $\operatorname{Ker} B_{1}=0$, i.e.: é uma forma bilinear não degenerada. Podemos definir uma plicação $A: V \rightarrow V$ como $B_{1}(A(u))=B_{2}(u)$ que está bem definida por $B_{1}$ ser não degenerada. Agora nós temos, para $u, v \in V, g \in G$ :

$$
\langle A \rho(g)(u), \rho(g) v\rangle_{1}=\langle\rho(g)(u), \rho(g) v\rangle_{2}=\langle u, v\rangle_{2}=\langle A(u), v\rangle_{1}=\langle\rho(g) A(u), \rho(g) v\rangle_{1}
$$

portanto $A$ comuta com todos os operadores $\rho(g)$ e portanto pelo Lema de Schur, é um múltiplo da identidade. Portanto $\langle\cdot, \cdot\rangle_{1}=c\langle\cdot, \cdot\rangle_{2}$, e, fazendo $V \equiv \mathfrak{g}, \rho=$ Ad e $B_{1}=K \mathrm{e}$ $B_{2}=$ tr podemos assumir a métrica mais tratável em $\mathfrak{g}$ :

$$
\langle h, j\rangle=-\operatorname{tr}(h j)
$$

que, como já mostramos, também é invariante pela ação adjunta de representações lineares de $G$, e portanto é um múltiplo da forma de Killing.

Voltemos agora a considerar o fibrado associado $P_{F}$. É claro que podemos (no caso $\mathfrak{u}(n)$ ) induzir uma forma bilinear simétrica em $P_{F}$ pelo traço em $F=\operatorname{End}(V)$, já que por (3.1) o traço é invariante pela ação de $\operatorname{Aut}_{G}(F)$. Como mostramos também na última seção do capítulo anterior (2.52), qualquer $G$-conexão advinda de $P$ será automaticamente compatível com esse produto interno. 
Pela ótica do fibrado vetorial $\operatorname{End}_{G}(E)$, definimos igualmente o traço de uma seção de End $(E)$, e para $\lambda \otimes T \in \Gamma\left(\left.\Lambda^{p}\left(\left.T M^{*}\right|_{\theta}\right) \otimes \operatorname{End}(E)\right|_{\theta}\right)$ definimos: $\operatorname{tr}(\lambda \otimes T)=\operatorname{tr}(T) \lambda \in$ $\Gamma\left(\Lambda^{p}\left(\left.T M^{*}\right|_{\theta}\right)\right.$. É claro que, como $\operatorname{End}\left(E_{x}\right) \simeq E_{x} \otimes E_{x}^{*}$, uma seção $s \in \Gamma(\operatorname{End}(E))$ sob um isomorfismo de fibrados $g \in \Gamma\left(\operatorname{Aut}_{G}(E)\right)$ se transforma pela conjugação : $s(x) \rightarrow$ $g^{-1}(x) s(x) g(x) \in \operatorname{End}\left(E_{x}\right)$. Portanto a ação do traço é invariante por uma tal transformação (o que poderia ser visto de outra maneira, notando que o traço é uma aplicação $C^{\infty}(M, \mathbb{R})$ bilinear $E^{*} \times E \rightarrow \mathbb{R}$, ou seja, é um tensor de ordem $(1,1)$, e portanto invariante por mudanças de base, ou isomorfismos agindo pela conjugação).

Assim, o fibrado $\operatorname{End}_{G}(E)$ tem um produto interno canônico sobre cada fibra que é preservado por transformações de gauge, que como sabemos, agem pela conjugação. Agora, induzimos uma métrica em $\operatorname{End}_{G}(E)$ pelas métricas canônicas das trivializações. Sem utilizar as formas de conexão, veremos como este produto interno é compatível com a conexão.

Sejam $\phi, \psi$ duas trivializações sobre $\theta$, i.e.:

$$
\pi_{E}^{-1}(\theta) \stackrel{\phi, \psi}{\simeq} \theta \times \operatorname{End}(V)
$$

Lembramos que as bases $G$-admissíveis de $\operatorname{End}(E)$ foram definidas como aquelas provindas das bases canônicas das trivializações e que, para $x \in \theta, v \in \operatorname{End}(V)$ a transição é dada por

$$
\phi \circ \psi^{-1}(x, v)=\left(x, g_{\phi \psi}(x) v g_{\phi \psi}(x)^{-1}\right)
$$

onde $\operatorname{Ad}\left(g_{\phi \psi}\right): \theta \rightarrow \operatorname{Aut}(\operatorname{End}(V))$. Para que faça sentido induzirmos a métrica em $\operatorname{End}(E)$ pelas métricas das trivializações, nós precisamos que $\operatorname{Ad}\left(g_{\phi \psi}\right): \theta \rightarrow \mathbf{O}(\operatorname{End}(V)) \subset \operatorname{Aut}(\operatorname{End}(V))$, agora

$$
\left\{\psi^{-1}\left(x, \tilde{e}_{i}^{j}\right)\right\}_{i, j=1}^{k}=\left\{e_{i}^{j}\right\}_{i, j=1}^{k}
$$

onde $\tilde{e}_{i}^{j}=\tilde{e}^{j} \otimes \tilde{e}_{i}$ é a base canônica de $V \otimes V^{*}$ e portanto $\left\{e_{i}^{j}\right\}_{i, j=1}^{k}$ é base ortonormal em $\operatorname{End}\left(E_{x}\right) ; \operatorname{logo}\left\{\phi\left(e_{i}^{j}\right)\right\}_{i, j=1}^{k}=\left\{\left(x, g_{\phi \psi}(x) e_{i}^{j} g_{\phi \psi}(x)^{-1}\right)\right\}_{i=1}^{k}$ é ortonormal.

Sejam então $x, y \in \theta$, e suponhamos que $\alpha: I \rightarrow \theta$ é uma curva tal que $\alpha(0)=x$ e $\alpha(1)=y$. Como o transporte paralelo leva base admissível am base admissível, nós temos que existem trivializações $\phi, \psi$, tais que se $\left\{\psi^{-1}\left(x, \tilde{e}_{i}^{j}\right)\right\}_{i, j=1}^{k}=\left\{e_{i}^{j}\right\}_{i, j=1}^{k}$ é base ortonormal em $\operatorname{End}\left(E_{x}\right)$, então

$$
\left\{b_{i}^{j}\right\}_{i, j=1}^{k}=\left\{P_{\alpha}\left(e_{i}^{j}\right)\right\}_{i, j=1}^{k}=\left\{\phi^{-1}\left(y, \tilde{e}_{i}^{j}\right)\right\}_{i, j=1}^{k}=\left\{\psi^{-1}\left(y, g_{\phi \psi}(y) \tilde{e}_{i}^{j} g_{\phi \psi}(y)\right)^{-1}\right\}_{i, j=1}^{k}
$$

que é ortonormal. Portanto (ao menos dentro de uma trivialização) o transporte paralelo leva base ortonormal em base ortonormal. Da mesma forma obtemos que será invariante por qualquer métrica em $\operatorname{End}(E)$ induzida por trivializações locais. Apesar de termos considerado curvas restritas a um domiínio trivial (precisaríamos ainda colar todas as partes da curva), esta descrição, como não poderia deixar de ser, é completamente equivalente a feita diretamente pelo fibrado associado.

Suponhamos agora que $\omega^{s}=s^{*} \omega$, onde $\omega$ é a forma de conexão em $P$ e $s$ é uma seção de $P$, como sabemos essa conexão terá uma representação no fibrado associado, o que nos incita uma pergunta, ainda que periférica ao estudo que estamos conduzindo, será que a representação de uma forma de conexão em $\mathrm{P}$ (que tem valores em $\mathfrak{g}$ ) é compatível com a conexão usual no fibrado vetorial $\operatorname{End}(E)$, dado por $((1.86),(1.84))$ ? Caso a construção que 
fizemos até agora esteja coerente, a resposta deve ser positiva. Chequemos pois que isso se dá.

Dem: Tomamos a representação $\rho_{x}: G \rightarrow \operatorname{Aut}\left(E_{x}\right) \mathrm{e}^{5}$ então, como sabemos, a respectiva representação do grupo que estamos tomando sobre $\operatorname{End}(E)$ (ou, no contexto de fibrado associado, sobre $\operatorname{End}(V))$ é :

$$
\begin{aligned}
\tilde{\rho}_{x}: G & \longrightarrow \operatorname{Aut}\left(\operatorname{End}\left(E_{x}\right)\right) \\
g & \longmapsto \operatorname{Ad}_{\rho_{x}(g)}
\end{aligned}
$$

Consideremos a aplicação Ad $: G \rightarrow \operatorname{Aut}(\mathfrak{g})$. Chamando $d(\operatorname{Ad})_{e}=\operatorname{ad}: \mathfrak{g} \rightarrow \operatorname{End}(\mathfrak{g})$, temos que

$$
d\left(\tilde{\rho}_{x}\right)_{e}=d\left(\operatorname{Ad}_{\rho_{x}(g)}\right)_{e}=\operatorname{ad}_{d\left(\rho_{x}\right)_{e}(h)}
$$

sendo que consistentemente $\left(d \rho_{x}\right)_{e}: \mathfrak{g} \rightarrow \operatorname{End}\left(E_{x}\right)$. Portanto nós temos o mapa

$$
\begin{aligned}
& d\left(\tilde{\rho}_{x}\right)_{e}: \mathfrak{g} \longrightarrow \operatorname{End}\left(\operatorname{End}\left(E_{x}\right)\right) \\
& h \longmapsto \operatorname{ad}_{d\left(\rho_{x}\right)_{e}(h)}
\end{aligned}
$$

Agora, é possível mostrar [16] que

$$
\begin{aligned}
\operatorname{ad}_{h}: & \mathfrak{g} \\
j & \longmapsto[h, j]
\end{aligned}
$$

Portanto para $X \in \Gamma(E)$ e $\lambda \in \Gamma\left(E^{*}\right)$,

$$
d\left(\tilde{\rho}_{x}\right)_{e}\left(\omega^{s}\right)(X \otimes \lambda)=\operatorname{ad}_{d\left(\rho_{x}\right)_{e}\left(\omega^{s}\right)}(X \otimes \lambda)=d\left(\rho_{x}\right)_{e}\left(\omega^{s}\right) X \otimes \lambda-X \otimes \lambda d\left(\rho_{x}\right)_{e}\left(\omega^{s}\right)
$$

e como

$$
\lambda d\left(\rho_{x}\right)_{e}\left(\omega^{s}\right)=\left(d\left(\rho_{x}\right)_{e}\left(\omega^{s}\right)\right)^{T} \lambda
$$

reinserindo em (3.2) nós obtemos a forma usual da conexão para o fibrado $E \otimes E^{*}((1.86),(1.84))$ (através da forma de conexão em $P$ pela representação natural de $\mathfrak{g}$ em $\operatorname{End}\left(E_{x}\right)$ ).

Ou seja, ao invés de tomarmos o produto interno na fibra sobre $x \in M$ como $(h, j) \mapsto$ $\operatorname{tr}\left(\operatorname{ad}_{d\left(\rho_{x}\right)_{e}(h)} \operatorname{ad}_{d\left(\rho_{x}\right)_{e}(j)}\right)$, faremos $(h, j) \mapsto \operatorname{tr}\left(d\left(\rho_{x}\right)_{e}(h) d\left(\rho_{x}\right)_{e}(j)\right)$.

\section{Equação de Yang-Mills}

Chamaremos de $\operatorname{Ad}(E) \subset \operatorname{End}(E)$ o espaço de endomorfismos cujas representações locais em matrizes são anti-simétricas em cada fibra $E_{x}$, i.e.: que são representações em $\mathfrak{s o}\left(E_{x}\right)$.

Nós já temos um produto interno para $p$-formas definido por, para $w_{1}, w_{2} \in \Lambda^{p} T_{x}^{*} M$ :

$$
\left\langle w_{1}, w_{2}\right\rangle * 1=w_{1} \wedge * w_{2}
$$

Logo, sejam

$$
\mu_{1} \otimes w_{1}, \mu_{2} \otimes w_{2} \in \operatorname{Ad}\left(E_{x}\right) \otimes \Lambda^{p} T_{x}^{*} M
$$

\footnotetext{
${ }^{5} \mathrm{Na}$ maioria dos casos, se usa a identificação $\operatorname{Aut}\left(E_{x}\right) \cong \mathbf{G L}(k)$ e toma-se a representação de $G$ simplesmente como a inclusão, $G \hookrightarrow \mathbf{G L}(k)$, mas não o faremos aqui.
} 
Então

$$
\left\langle\mu_{1} \otimes w_{1}, \mu_{2} \otimes w_{2}\right\rangle:=\operatorname{tr}\left(\mu_{1} \mu_{2}\right)\left\langle w_{1}, w_{2}\right\rangle
$$

ou seja, temos um produto escalar $L^{2}$ em $\Gamma\left(\Lambda^{p}(T M) \otimes \operatorname{Ad} E\right)$ :

$$
\left\langle\left\langle\mu_{1} \otimes w_{1}, \mu_{2} \otimes w_{2}\right\rangle\right\rangle:=\int_{M}\left\langle\mu_{1} \otimes w_{1}, \mu_{2} \otimes w_{2}\right\rangle * 1
$$

Logo, como temos um produto interno bem definido, podemos definir o operador estrela de Hodge neste espaço também. Nós temos ainda que, a adjunta formal da derivada covariante é dada por $D^{*}=(-1)^{n(p+1)+1} * D *$. Este fato pode ser facilmente demonstrável utilizando o Gauge canônico, já que ali

$$
\tilde{D} \alpha\left(x_{1}, \cdots, x_{p+1}\right)=d \alpha\left(x_{1}, \cdots, x_{p+1}\right)
$$

e nós já demonstramos que $d^{*}=(-1)^{n(p+1)+1} * d *$.

Assumindo ainda que $D$ é uma derivada de gauge exterior compatível com a métrica $(D$ é compatível com $\langle\cdot, \cdot\rangle$ se $D\langle\mu, \nu\rangle=d\langle\mu, \nu\rangle=\langle D \mu, \nu\rangle+\langle\mu, D \nu\rangle$ ou se a forma de conexão tem representação anti-simétrica) com curvatura $\Omega_{D} \in \Gamma\left(\Lambda^{2}(T M) \otimes \operatorname{Ad} E\right)$, utilizando (3.3) definimos o funcional de Yang-Mills aplicado a $D$ :

$$
S_{Y M}(D):=\left\langle\left\langle\Omega_{D}, \Omega_{D}\right\rangle\right\rangle=\int_{M}\left\langle\Omega_{D}, \Omega_{D}\right\rangle * 1=\int_{M} \operatorname{tr}\left(\Omega_{D} \wedge * \Omega_{D}\right)
$$

O integrando $\left\langle\Omega_{D}, \Omega_{D}\right\rangle=L_{Y M}$ é chamado de Lagrangeana de Yang-Mills e $S_{Y M}(D)$ é chamada de ação de Yang-Mills (ou funcional de Yang-Mills). Como vimos, uma transformação de Gauge deixa $L_{Y M}$ e portanto $S_{Y M}$ invariantes. Para determinarmos as equações de Euler-Lagrange para esse funcional utilizamos o fato que o espaço de todas as conexões métricas em $E, \mathcal{C}(E)$ é um espaço afim. Portanto, podemos considerar variação da forma: $D+t \gamma$ onde $\gamma \in \Gamma\left(\Lambda^{1}(T M) \otimes \operatorname{Ad}(E)\right)$ Agora por (1.118):

$$
\Omega_{D+t \gamma}=\Omega_{D}+t D \gamma+t^{2} \gamma \wedge \gamma
$$

logo

$$
\left.\frac{d}{d t}\right|_{t=0} S_{Y M}(D+t \gamma)=\left.\frac{d}{d t}\right|_{t=0}\left(\int\left\langle\Omega_{D+t \gamma}, \Omega_{D+t \gamma}\right\rangle * 1\right)=2 \int\left\langle\Omega_{D}, D \gamma\right\rangle * 1=2\left\langle\left\langle D^{*} \Omega_{D}, \gamma\right\rangle\right\rangle
$$

assim, $D$ extremiza $S_{Y M}$ se $D^{*} \Omega_{D}=0$, onde $D^{*}=* D *$. Chamamos tal $D$ de conexão de Yang-Mills. Então, levando em conta a identidade de Bianchi (1.72), temos que a conexão de Yang-Mills deve obedecer às seguintes equações:

$$
\left\{\begin{array}{l}
D * \Omega_{D}=0 \\
D \Omega_{D}=0
\end{array}\right.
$$

Além disso, denotando por $\delta \Omega$ a variação de $\Omega$, como $\delta \omega=\gamma$ por (3.5), emerge gratuitamente que,

$$
\delta \Omega=D \delta \omega
$$


Portanto, como $\Omega=D \omega$ nós obtemos que a derivada funcional e a covariante comutam para a forma de conexão.

Além da invariância de Gauge, as equações de Yang-Mills têm outras propriedades interessantes advindas de simetrias em $L_{Y M}$. Como mencionamos algumas vezes, o produto interno induzido sobre a fibra é invariante por automorfismos do fibrado, e se olharmos bem, notamos que a métrica de $M$ entra de forma muito sutil nas equações, somente através do operador de Hodge (já que $\Omega_{D}$ só depende da forma de conexão $\omega$ ), pela equação (1.45).

Seja $g$ uma métrica em $M$, tomamos uma métrica conformemente equivalente a $g, \tilde{g}=f^{2} g$ para $0 \neq f \in C^{\infty}(M, \mathbb{R})$. É fácil ver que se $\left\{e_{I}\right\}$ é base ortonormal de $\Lambda^{p}\left(T^{*} M\right)$ em relação a $g$, então $\left\{\frac{e_{I}}{f}\right\}$ o é em relação a $\tilde{g}$. Logo, como $\tilde{*}_{p}$ está definido por sua aplicação a uma base de $\Lambda^{p}\left(T^{*} M\right)$, para cada elemento de $\left\{e_{I}\right\}$ temos

$$
\begin{gathered}
\tilde{*}\left(\tilde{e}_{i_{1}} \wedge \cdots \wedge \tilde{e}_{i_{p}}\right)= \pm \wedge \tilde{e}_{i_{p+1}} \wedge \cdots \wedge \tilde{e}_{i_{n}} \\
=\frac{1}{f^{p}} \tilde{*}\left(e_{i_{1}} \wedge \cdots \wedge e_{i_{p}}\right)= \pm \frac{1}{f^{n-p}} e_{i_{p+1}} \wedge \cdots \wedge e_{i_{n}} \\
\therefore \quad \tilde{*}\left(e_{i_{1}} \wedge \cdots \wedge e_{i_{p}}\right)= \pm f^{2 p-n} e_{i_{p+1}} \wedge \cdots \wedge e_{i_{n}}=f^{2 p-n} *\left(e_{i_{1}} \wedge \cdots \wedge e_{i_{p}}\right)
\end{gathered}
$$

E finalmente

$$
\tilde{*}=f^{2 p-n} *
$$

Logo se $n=2 p$ obtemos $\tilde{*}=*$.

Isso significa que se $\Psi: M^{m} \rightarrow N^{n}$ é um difeomorfismo entre $(M, \tilde{g})$ e $(N, g)$ tal que $m=n=2 p$ e se para alguma função $f$, onde $0 \neq f \in C^{\infty}(M, \mathbb{R})$ nós tenhamos $\Psi^{*} g=f^{2} \tilde{g}$, então para todo $w \in \Lambda^{p}\left(T^{*} N\right)$, temos:

$$
\Psi^{*}(* w)=\tilde{*}\left(\Psi^{*} w\right)
$$

O que é facilmente obtido de (3.7) fazendo a seguinte substituição $\left\{\frac{e_{I}}{f}\right\} \rightarrow\left\{\frac{\Psi^{*} e_{I}}{f}\right\}$.

Agora nós sabemos que dado $\nabla \in C(E)$, onde $\pi: E \rightarrow N$, nós formamos o pull-back $\phi^{*}(E)$ sobre $M$ e obtemos uma conexão canônica em $\phi^{*}(E)$ dada pelas formas de conexão

$$
\tilde{w}=\phi^{*} w
$$

Logo, lembrando que para todo $\lambda \in \Gamma\left(\Lambda^{p}\left(T^{*} M\right) \otimes \operatorname{End}(E)\right)$ por (1.115) temos

$$
D \lambda=d \lambda+w \wedge \lambda
$$

vale:

$$
\phi^{*}(D \lambda)=\phi^{*} d \lambda+\phi^{*} w \wedge \phi^{*} \lambda=d\left(\phi^{*} \lambda\right)+\tilde{w} \wedge \phi^{*} \lambda=\tilde{D}\left(\phi^{*} \lambda\right)
$$

e portanto para o difeomorfismo $\Psi$, por (3.8) e tomando uma conexão de Yang-Mills em $E$ :

$$
\Psi^{*}(D * \Omega)=\tilde{D}\left(\Psi^{*} * \Omega\right)=\tilde{D} \tilde{*}\left(\Psi^{*} \Omega\right)=\tilde{D} \tilde{*} \tilde{\Omega}=0
$$

e a igualdade de Bianchi também vale automaticamente, obtemos que se $D$ é conexão de Yang-Mills, $\tilde{D}$ também o é. Um caso particular 'e se um difeomorfismo $\Phi: M \rightarrow M$ for uma isometria, $\Phi^{*}$ preserva o operador de Hodge e portanto preserva $L_{Y M}$ e as equações de Yang-Mills. Por exemplo, as equações de Yang-Mills para o fibrado trivial são invariantes pelo grupo de Poincaré. 
Mas explorando essa maior liberdade conforme, como a métrica canônica de $S^{4}-\{p\}$ é conforme a de $\mathbb{R}^{4}$ sob projeção estereográfica existe uma correspondência bijetora entre campos Yang-Mills $\left(\Omega_{D}\right)$ em $\mathbb{R}^{4}$ e em $S^{4}-\{p\}$.

É claro que qualquer campo de Yang-Mills em $S^{4}$ tem ação finita e portanto corresponde a um campo de Yang-Mills de ação finita em $\mathbb{R}^{4}$ :

$$
\int_{s^{4}} \operatorname{tr}(\Omega \wedge * \Omega)=\int_{S^{4}-\{p\}} \operatorname{tr}(\Omega \wedge * \Omega)=\int_{\mathbf{R}^{4}} \operatorname{tr}\left(\Psi^{*} \Omega \wedge * \Psi^{*} \Omega\right)=\int_{\mathbf{R}^{4}} \operatorname{tr}(\tilde{\Omega} \wedge * \tilde{\Omega})
$$

Um teorema de Karen Uhlenbeck nós fornece a recíproca: um campo $Y M$ de ação finita em $\mathbb{R}^{4}$ se estende a um campo $Y M$ suave em $S^{4}$. Portanto, os campos $Y M$ de ação finita em $R^{4}$ podem ser identificados com todos os campos $Y M$ de $S^{4}$.

No caso mais geral de $n=4$, como vimos na teoria de Hodge, como $\left(*_{2}\right)^{2}=1$ por $(1.42)$, podemos tomar a soma direta

$$
\Lambda^{2}\left(T^{*} M\right) \otimes \operatorname{End}(E)=\Lambda_{+}^{2}\left(T^{*} M\right) \otimes \operatorname{End}(E) \oplus \Lambda_{+}^{2}\left(T^{*} M\right) \oplus \operatorname{End}(E)
$$

de subespaços de autovetores de $*$. Em particular,

$$
\Omega=\Omega_{+}+\Omega_{-}
$$

e automaticamente satisfazemos a equação de Yang-Mills: $D * \Omega=D \Omega=0$. Chamamos uma forma de conexão $\omega$ de auto-dual (anti-dual) se sua sua curvatura é $\Omega_{+}\left(\Omega_{-}\right)$. Normalmente essas conexões são designadas por e $S D$ (self-dual) e $A S D$ (anti-self-dual). É ainda fácil ver que essa decomposição é ortogonal:

$$
\left\langle\left\langle\Omega_{+}, \Omega_{-}\right\rangle\right\rangle=\int_{M} \Omega_{+} \wedge * \Omega_{-}=-\int_{M} * \Omega_{+} \wedge \Omega_{-}=-\int_{M} \Omega_{-} \wedge * \Omega_{+}=-\left\langle\left\langle\Omega_{-}, \Omega_{+}\right\rangle\right\rangle=0
$$

Soluções duais da equação de Yang-Mills são chamadas de instantons, e existe uma vasta quantidade de física e matemática que revolvem ao seu redor. Um exemplo de uma solução dual não trivial (i.e.: com curvatura não nula) de energia finita é

$$
\omega_{x}=\operatorname{im}\left(\frac{\bar{x} d x}{1+|x|^{2}}\right)
$$

onde $x$ é a variável quaterniônica em $\mathbb{R}^{4} \simeq \mathbb{H}$ e identificamos a álgebra de Lie $\mathbf{S U}(2) \simeq \mathbf{S p}(1)$ ao conjunto de quaternions imaginários [13]. Em 1979, fórmulas similares foram descobertas para todas as conexões duais e anti-duais em $\mathbb{R}^{4}$ euclidiano [11].

Durante algum tempo, as únicas soluções de energia finita conhecidas para as equações de Yang-Mills eram soluções duais e anti-duais. Em 1989, L.Sibner, R. Sibner e K. Uhlembeck [12] publicaram uma prova da existência de soluções de algumas soluções não duais no $\mathbb{R}^{4}$ euclidiano. Uma outra boa referência para o assunto é [13].

Exploraremos um pouco melhor a relação da equação de Yang-Mills com a topologia e classificação de fibrados na próxima seção. 


\subsection{Classificação de Fibrados}

Nesta seção estaremos preocupados em achar maneiras de dizer quando dois fibrados sobre a mesma base são ou não são isomorfos, questão encontrada no Teorema de Classificação de Fibrados Vetoriais, um resultado de ordem formal. Na verdade, o que procuramos é uma maneira de associar a cada fibrado vetorial $E$ sobre uma variedade base $M$, uma classe de cohomologia de $M$. A essas associações damos o nome de classes características, e elas são invariantes globais e pode-se dizer que medem o desvio de uma estrutura produto global. Classes características é um dos conceitos geométricos que conecta topologia algébrica e geometria diferencial, e apesar de não fazermos tanto uso destas construções neste trabalho, sua importância não pode ser exagerada para a física moderna. Não faremos no entanto uma apresentação formal, mesmo dos poucos tópico que abordamos, já que exigiria uma bagagem algébrica que excederia nosso tempo disponível e conhecimento da área.

\section{* Topologia de Fibrados Vetoriais}

Apresentaremos primeiramente uma introdução aos resultados mais elementares sobre a topologia de fibrados vetoriais.

Denotamos por $\operatorname{Vect}_{G}(M)$ o conjunto de classes de equivalência de $G$-fibrados sobre $M$, isto é, $E \sim F$ se e somente se existe difeomorfismo $\Psi: E \rightarrow F$ tal que $\pi \circ \Psi=\pi$ isto é, $\Psi$ é um isomorfismo de fibrados sobre $M$. É trivial verificar que isso realmente constitui uma relação de equivalência.

Dado $f: N \rightarrow M$, construímos o mapa induzido pelo pull-back:

$$
f^{*}: \operatorname{Vect}_{G}(M) \rightarrow \operatorname{Vect}_{G}(N)
$$

Lembramos que se $E$ é fibrado vetorial sobre $M$, então

$$
f^{*}(E):=\left\{(x, v) \mid x \in N, v \in E_{f(x)}\right\}
$$

Para verificar que este mapa está bem definido sobre as classes de equivalência, basta vermos que, se $E \stackrel{\Psi}{\simeq} F$, ambos fibrados vetoriais sobre $M$, então o mapa

$$
\begin{aligned}
& f^{*}(E) \longrightarrow f^{*}(F) \\
& (x, v) \longmapsto(x, \Psi(v))
\end{aligned}
$$

é isomorfismo de fibrados. Dado $E$, denotamos $E \times I$ o fibrado sobre $N \times I$, onde a fibra sobre $(x, t)$ é a fibra de $E$ sobre $x$. Isto é,

$$
E \times I=\operatorname{pr}_{1}^{*}(E)
$$

onde

$$
\operatorname{pr}_{1}: N \times I \rightarrow N
$$

* Proposition 19 Todo $G$-fibrado $\widetilde{E}$ sobre $N \times I$ é isomorfo a um da forma $E \times I$ (ou seja $\left.p r_{1}^{*} E\right)$, para algum fibrado $E$ sobre $N$. 
Dem: Seja $\widetilde{E}$, tomamos

$$
\begin{aligned}
\imath_{o}: & N \rightarrow N \times I \\
& x \mapsto(x, 0)
\end{aligned}
$$

definimos então $E=\imath_{o}^{*}(\widetilde{E})$, ou seja $E_{x}=\widetilde{E}_{\imath_{o}(x)}=\widetilde{E}_{(x, 0)}$. Agora, tomando o fibrado $E \times I$ definido acima temos:

$$
(E \times I)_{(x, t)}=E_{\mathrm{pr}_{1}(x, t)}=E_{x}=\widetilde{E}_{(x, 0)}
$$

Escolhida uma conexão em $\widetilde{E}$, seja $P_{(x, t)}: \widetilde{E}_{(x, 0)} \rightarrow \widetilde{E}_{(x, t)}$ o transporte paralelo ao longo da curva $t \rightarrow(x, t)$.

Logo, fazendo $(E \times I)_{(x, t)} \equiv E_{x} \equiv \widetilde{E}_{(x, 0)}$ basta tomarmos

$$
\begin{array}{cl}
\phi_{(x, t)}:(E \times I)_{(x, t)} & \rightarrow \widetilde{E}_{(x, t)} \\
v & \mapsto P_{(x, t)}(v)
\end{array}
$$

como o transporte paralelo é um isomorfismo entre as fibras, e depende suavemente dos parâmetros $(x, t)$, nós temos que $\phi: E \times I \rightarrow \widetilde{E}$ é um isomorfismo de fibrados. Sua ação explora a estrutura produto da base e equivale a transladar as fibras por transporte paralelo ao longo de cada reta $t \mapsto(x, t)$.

*Theorem 26 Se $f_{0}, f_{1}: N \rightarrow M$ são homotópicas, então $f_{0}^{*}: \operatorname{Vect}_{G}(M) \rightarrow \operatorname{Vect}_{G}(N) e$ $f_{1}^{*}: \operatorname{Vect}_{G}(M) \rightarrow \operatorname{Vect}_{G}(N)$ são iguais.

Dem: Seja $E \in \operatorname{Vect}_{G}(M)$, e $F: N \times I \rightarrow M$ uma homotopia entre $f_{0}$ e $f_{1}$. Então $F^{*}(E)$ é fibrado sobre $N \times I$. Logo, para algum $\widetilde{E}$ sobre $N$,

$$
F^{*}(E) \simeq \widetilde{E} \times I
$$

Portanto, definindo $f_{t}:=F \circ \imath_{t}: N \rightarrow M$, onde

$$
\begin{aligned}
\imath_{t}: & N \rightarrow N \times I \\
& x \mapsto(x, t)
\end{aligned}
$$

nós temos que $f_{t}^{*}=\imath_{t}^{*} \circ F^{*}$. Logo, como

$$
F^{*} E \simeq \widetilde{E} \times I \text { e } \imath_{t}^{*}: \operatorname{Vect}_{G}(N \times I) \rightarrow \operatorname{Vect}_{G}(N)
$$

estão bem definidas,

$$
f_{t}^{*}(E) \simeq \imath_{t}^{*}(\widetilde{E} \times I)=\widetilde{E}
$$

Então, $f_{0}^{*}(E) \simeq f_{1}^{*}(E)$.

Proposition 20 Se $M$ é contrátil, então todo G-fibrado sobre $M$ é trivial.

Dem: Seja $f_{0}=\operatorname{Id}_{M}$ e $f_{1}: M \rightarrow\{p\} \in M$ mapa constante; seja $E$ um fibrado qualquer sobre $M$. Então, como $f_{0}$ é homotópica a $f_{1}$, e $f_{0}^{*}=\operatorname{Id}_{E}$, obtemos pelo teorema anterior que

$$
E=f_{0}^{*}(E) \simeq f_{1}^{*}(E)=M \times E_{p}
$$


Proposition 21 Nós temos um isom.orfismo $\operatorname{Vect~}_{G}\left(S^{n}\right) \rightarrow \pi_{n-1}(G)$.

Dem.: Um $G$-fibrado $F$ sobre $S^{n}$ é equivalente a um fibrado formado pela colagem (como vimos na Sec.1.1) dos fibrados $E^{+}$e $E^{-}$sobre os hemisférios norte $D_{+}^{n}$ e sul $D_{-}^{n}$ ao longo do equador $S^{n-1}=D_{+}^{n} \cap D_{-}^{n}$ por um mapa de transição de gauge $g: S^{n-1} \rightarrow G$. Pelo corolário anteriror $E^{+}$e $E^{-}$são triviais. Isso significa que temos a liberdade de tomar as trivializações

$$
\psi_{1}: E_{\mid D_{+}^{n}} \rightarrow D_{+}^{n} \times E_{p} \quad, \quad \psi_{2}: E_{\mid D_{-}^{n}} \rightarrow D_{-}^{n} \times E_{p}
$$

Portanto, como $\psi_{1} \circ \psi_{2}^{-1}:\left(D_{+}^{n} \times E_{p}\right)_{D_{+}^{n} \cap D_{-}^{n}}=S^{n-1} \times E_{p}$

$$
\begin{aligned}
\psi_{1} \circ \psi_{2}^{-1}: & S^{n-1} \times E_{p} \rightarrow S^{n-1} \times E_{p} \\
& (x, q) \mapsto(x, g(x) \circ q)
\end{aligned}
$$

Logo, a classe de equivalência (de isomorfismos de fibrados) de $E$ é determinada unicamente pela classe de equivalência de $g: S^{n-1} \rightarrow G$, ou seja, nós obtemos um isomorfismo:

$$
\operatorname{Vect}_{G}\left(S^{n}\right) \rightarrow \pi_{n-1}(G)
$$

Exemplo: Como para um grupo $G$ simples e compacto $\pi_{3}(G)=\mathbb{Z}$, então $\operatorname{Vect}_{G}\left(S^{4}\right) \simeq$ $\pi_{3}(G)=\mathbb{Z}$.

\section{Teorema de Classificação de Fibrados Vetoriais}

A proposição 21 é na verdade um caso específico de um fato muito mais geral, o Teorema de Classificação de Fibrados. Este teorema prova que para qualquer grupo de Lie $G$ e inteiro positivo $N$, podemos construir um espaço $B_{G}=B_{G}^{N}$, chamado de espaço de classificação de $G$ para variedades $M$ de dimensão menor que $N$, e um $G$-fibrado vetorial sobre $B_{G}, \xi_{G}^{N}$, chamado de fibrado universal, tal que se $M$ for uma variedade suave de dimensão menor que $N$ e $E$ for qualquer $G$-fibrado vetorial sobre $M$, existe um mapa $f: M \rightarrow B_{G}$ tal que $E \simeq f^{*} B_{G}$. Para o caso $G=\mathbf{G L}(k)$, provaremos este teorema juntamente com sua extensão: se $E \simeq f_{0}^{*} \xi_{G}$ e $E \simeq f_{1}^{*} \xi_{G}$, então $f_{0}$ e $f_{1}$ são homotópicas.

Seja então

$$
Q^{r}=\left\{T \in L\left(\mathbb{R}^{k}, \mathbb{R}^{l}\right) \mid \operatorname{posto}(T)=r\right\}
$$

começamos com a seguinte proposição:

Proposition $22 Q^{r}$ tem dimensão $k l-(k-r)(l-r)$.

Dem: Consideremos a ação

$$
\begin{aligned}
\varphi: \mathbf{G L}(l) \times \mathbf{G L}(k) & \rightarrow \operatorname{Aut}\left(L\left(\mathbb{R}^{k}, \mathbb{R}^{l}\right)\right) \\
(g, h) & \mapsto I_{g_{g}} R_{h^{-1}}
\end{aligned}
$$

Afirmamos que as órbitas de $\varphi$ são justamente as transformações lineares de posto constante. Que elas mantém o posto constante é claro, vejamos porque todas as transformações de posto $r$ estão ligadas pela ação de $\varphi$ à matriz

$$
\left(\begin{array}{c|c}
\operatorname{Id}_{\mathbb{R}^{r}} & 0 \\
\hline 0 & 0
\end{array}\right)
$$


Tomando $T$ com posto $r, \operatorname{dim}(\operatorname{Ker} T)=k-r$. Tomamos $\left\{e_{i}\right\}_{i=r+1}^{k}$ como base de $\operatorname{Ker} T$, e um completamento qualquer $\left\{e_{i}\right\}_{i=1}^{r}$ em $\mathbb{R}^{k}$. Em $\mathbb{R}^{l}$, tomamos uma base composta por $\left\{T e_{i}\right\}_{i=1}^{r}=\left\{\tilde{e}_{i}\right\}_{i=1}^{r}$ e um completamento $\left\{\tilde{e}_{i}\right\}_{i=r+1}^{l}$. É claro que nessas bases $[T]$ será uma matriz diagonal com um único bloco $r \times r$ não nulo, justamente a identidade em $\mathbb{R}^{k}$. Como transformações de base em $\mathbb{R}^{k}$ e $\mathbb{R}^{l}$ são equivalentes a ação de $\varphi$, nós temos que $Q^{r}$ será dado pela órbita de $T$ pela ação de $\varphi$. Tomando as ações usuais de composição nos grupos $\operatorname{Aut}\left(L\left(\mathbb{R}^{k}, \mathbb{R}^{l}\right)\right)$ e $\mathbf{G L}(l) \times \mathbf{G L}(k)$, é trivial ver que $\varphi$ é um homomorfismo:

$$
\varphi\left(g g^{\prime}, h h^{\prime}\right) T=g\left(g^{\prime} T h^{\prime-1}\right) h^{-1}=\varphi(g, h) \varphi\left(g^{\prime}, h^{\prime}\right) T
$$

logo, $Q^{r} \simeq(\mathbf{G L}(l) \times \mathbf{G L}(k)) / G_{T}$ onde $G_{T}$ é o grupo de isotropia de $T$ pela ação de $\varphi$. Suponhamos então que $(\tilde{g}, \tilde{h}) \in G_{T}$. Temos então, utilizando a base acima para representar $T$ por (3.11), a seguinte condição

$$
T \circ h=\left(\begin{array}{lll}
h_{1}^{1} & \cdots & h_{1}^{k} \\
\vdots & & \vdots \\
h_{r}^{1} & \cdots & h_{r}^{k} \\
\hline & 0 &
\end{array}\right)=g \circ T=\left(\begin{array}{lll}
g_{1}^{1} & \cdots & g_{1}^{r} \\
\vdots & & \vdots \\
& & \\
g_{l}^{1} & \cdots & g_{l}^{r}
\end{array}\right)
$$

Para $h$, que é uma matriz quadrada $k \times k$, é fácil notar que só temos de fixar os termos $\left\{h_{j}^{i} \mid i=r+1, \cdots k\right.$, e $\left.j=1, \cdots r\right\}$ que têm de ser nulos pela igualdade à $g \circ T$ (temos liberdade para escolher os elementos que são levado em zero pela $T$ e o quadrado $r \times r$ esquerdo superior). Para a matriz $g \in \mathrm{M}(l \times l)$, com já fixamos $h$, precisamos fixar todos os elementos que não são levados a zero por $T$, ou seja todos os $r \times l$ elementos não nulos da matriz resultante. Isso nos dá uma dimensão de

$$
k r+r l-r^{2}=k l-\left(k l-k r-r l+r^{2}\right)=k l-(l-r)(k-r)
$$

Suponhamos, sem perda de generalidade, que $l \geq k$.

Proposition 23 Se $M$ é uma variedade suave com dimensão $m \leq(l-k)$, então para qualquer mapa $f: M \rightarrow L\left(\mathbb{R}^{k}, \mathbb{R}^{l}\right)$ que seja transversal a todos os $Q^{i}$, para $i<k$, e para todo $x \in M, f(x) \in L\left(\mathbb{R}^{k}, \mathbb{R}^{l}\right)$ terá posto $k$ constante.

Dem: pelo Teo.1, nós temos que $f: M \rightarrow L\left(\mathbb{R}^{k}, \mathbb{R}^{l}\right)$ é transversal a $Q^{i}$ se $Q^{i} \cap f(M)=\emptyset$ ou se

$$
\operatorname{Im}\left(d f_{x}\right)+T_{f(x)} Q^{i}=T_{f(x)} L\left(\mathbb{R}^{k}, \mathbb{R}^{l}\right)
$$

Em termos de dimensão, $(l-k)+\operatorname{dim}\left(Q^{i}\right) \geq \operatorname{dim}(M)+\operatorname{dim}\left(Q^{i}\right) \geq \operatorname{posto}\left(d f_{x}\right)+\operatorname{dim}\left(Q^{i}\right) \geq$ $\operatorname{dim}\left(L\left(\mathbb{R}^{k}, \mathbb{R}^{l}\right)\right.$. Portanto temos:

$$
(l-k)+k l-(l-i)(k-i) \geq \operatorname{posto}\left(d f_{x}\right)+k l-(l-i)(k-i) \geq l k
$$

Portanto

$$
(l-k) \geq \operatorname{posto}\left(d f_{x}\right) \geq(l-i)(k-i)
$$


Mas $l \geq k>i$. Logo chegamos a $(l-i)(k-i) \geq(l-i)>(l-k)$ c finalmentc,

$$
(l-k) \geq \operatorname{posto}\left(d f_{x}\right)>(l-k)
$$

absurdo. Portanto, para $\operatorname{dim} M \leq(l-k)$ e $f$ transversal a $Q^{i}$ nós temos $Q^{i} \cap f(M)=\emptyset \operatorname{logo}$ se isso é válido para todo $i<k$, chegamos a $f(M) \in Q^{k}$.

Antes de prosseguirmos, citamos um teorema sobre transversalidade [17]

Theorem 27 Sejam $M, N$ variedades suaves, $M$ variedade compacta, e $f_{0}: M \rightarrow N$ um mapa suave. Para qualquer $Z$ subvariedade mergulhada de $N$ temos os seguintes resultados:

Gen.: Existe homotopia $f_{t}: \Lambda \rightarrow N$ de $f_{0}$ tal que para $\epsilon>0$ suficientemente pequeno, $f_{c}$ é transversal a $Z$. Em outras palavras, podemos perturbar a função para que se torne transversal. Essa propriedade é chamada de genericidade.

Est.: Se $\int_{0}$ é transversal a $Z$, para qualquer homotopia suave $\int_{t}$, nós temos que $\int_{\epsilon}$ é transversal a $Z$ para $\epsilon$ suficientemente pequeno. Ou seja, ela se mantém transversal por pequenas perturbaçôes. Esta propriedade é chamada de estabilidade.

Cont.: Se $\int: M I \rightarrow N$ é suave e transversal à subvariedade mergulhada $Z$ em $p \in \Lambda I$, então existe aberto em torno de $p, U \subset M$ onde $f$ permanece transversal a $Z$.

Agora podemos enunciar a proposição chave:

Proposition 24 Se $M$ é compacto, então qualquer fibrado vetorial com fibra típica isomorfa $a \mathbb{R}^{k}$ é isomorfo a algum subfibrado do fibrado produto $\mathbb{R}_{M}^{l}:=M \times \mathbb{R}^{l}$, onde e $\operatorname{dim}(M)+k \leq l$.

Dem: Nós temos que $\pi_{L}: L\left(E, \mathbb{R}_{M}^{l}\right) \rightarrow M$ é um fibrado vetorial. Um isomorfismo de $E$ com um subfibrado de $\mathbb{R}_{M}^{l}$ pode ser visto então como uma seção $\Psi: M \rightarrow L\left(E, \mathbb{R}_{M}^{l}\right)$, no sentido que $\pi_{L} \circ \Psi=I d$ e tal que $\Psi_{x}: E_{x} \rightarrow \mathbb{R}^{l}$ é uma transformação linear de posto máximo, $k$, para todo $x \in M$. É uma tal seção que tentaremos construir.

Consideremos o fibrado vetorial $L\left(E, \mathbb{R}_{M}^{l}\right)$. Em uma trivialização

$$
U_{\alpha} \times\left. L\left(\mathbb{R}^{k}, \mathbb{R}^{l}\right) \stackrel{\phi_{\alpha}}{\simeq} L\left(E, \mathbb{R}_{M}^{l}\right)\right|_{U_{\alpha}}
$$

Agora tomamos a seção nula, que é uma seção global suave $\Psi_{0}: M \rightarrow L\left(E, \mathbb{R}_{M}^{l}\right)$. Sobre $U_{\alpha}$, nós tomamos o mergulho (das transformações de posto $i$ sobre $\alpha$ ):

$$
\phi_{\boldsymbol{\alpha}}\left(U_{\alpha} \times Q^{i}\right):=Z_{\alpha}^{i} \subset L\left(E, \mathbb{R}_{M}^{l}\right)
$$

Agora, por genericidade, existe homotopia de $\Psi_{0}$, que chamaremos de $\Psi_{\alpha}^{0}: M \times[0,1] \rightarrow$ $L\left(E, \mathbb{R}_{M}^{l}\right)$ que é transversal à subvariedade mergulhada $Z_{\alpha}^{0}$ em um dado $\left.\left.\epsilon_{0} \in\right] 0,1\right]$.

Agora tomamos uma homotopia de $\left(\Psi_{\alpha}^{0}\right)_{\epsilon_{0}}$, que chamamos de $\Psi_{\alpha}^{1}: M \times[0,1] \rightarrow L\left(E, \mathbb{R}_{M}^{l}\right)$. Por genericidade esta homotopia é transversal a $Z_{\alpha}^{1}$ para algum $\left.\left.\epsilon_{1} \in\right] 0,1\right]$ suficientemente pequeno para que, por estabilidade, continue sendo transversal a $Z_{\alpha}^{0}$. Procedendo dessa forma $k$ vezes obtemos uma seção global, transversal a todos os $Q^{i}$ sobre $U_{\alpha}$ (para $i<k$ ). Logo pela proposição anterior, esta seção tem posto $k$ sobre $U_{\alpha}$. 
Então, como $M$ foi assumido compacto, utilizamos exatamente o mesmo método (atentando para mantermos as perturbações sobre o domínio da estabilidade) um número finito de vezes, obtendo uma seção $\Psi$ de $L\left(E, \mathbb{R}_{M}^{l}\right)$ de posto $k$, ou seja, nós temos uma imersão de $E$ sobre uma $k$-distribuição em $M \times \mathbb{R}^{l}$.

Notemos que o único caso em que este teorema permite que trivializemos $E$, é para $l=k$ e portanto $\operatorname{dim} M=0$, ou seja, para $M=\{p\}$.

Agora, suponhamos que $E$ seja um fibrado sobre $N$, e $M$ seja subvariedade mergulhada fechada de $N$. Seja $\imath^{*}(E)=\left.E\right|_{M}$ o fibrado sobre $M$, e suponha que já temos uma seção $\Psi: M \rightarrow L\left(E, \mathbb{R}_{M}^{l}\right)$ tal que $\Psi(x)$ tenha posto máximo para todo $x \in M$.

Proposition 25 Existe seção $\zeta: N \rightarrow L\left(E, \mathbb{R}_{N}^{l}\right)$ de posto máximo tal que $\Psi=\left.\zeta\right|_{M}$.

Dem: Agora, ao invés da seção nula que tomamos no início da demonstração acima, tentaremos construir uma seção suave $\zeta$ tal que $\left.\zeta\right|_{M}=\Psi$, i.e.: $\zeta$ é uma extensão suave qualquer de $\Psi$.

Como $M$ é subvariedade mergulhada de $N$, temos ao redor de cada ponto $p \in M$ um aberto $U_{p}$ (pequeno suficiente para que seja domínio de uma trivialização de $E$ ), domínio de coordenadas cúbicas $\eta: U_{p} \rightarrow \mathbb{R}^{n}$ centradas em $p$ tal que $M \cap U_{p}=S_{p}$ é uma única fatia. Logo podemos tomar uma projeção suave $\pi_{U_{p}}: U_{p} \rightarrow S_{p}$ (podemos definir $\pi_{p}=\eta^{-1} \circ \mathrm{pr}_{m} \eta$ ) e assim definimos

$$
\bar{\zeta}_{p}:=\Psi \circ \pi_{p}: U_{p} \rightarrow L\left(\mathbb{R}^{k}, \mathbb{R}^{l}\right)
$$

Agora, a coleção $\left\{U_{p} \mid p \in M\right\} \cup(N-M)$ forma uma cobertura aberta de $N$, logo podemos tomar partição da unidade $\left\{\sigma_{i} \mid i=1,2, \cdots\right\}$ subordinada a essa cobertura. Tomando a subsequência $\left\{\sigma_{i} \mid \operatorname{supp} \sigma_{i} \cap M \neq \emptyset\right\}$, para cada tal $i$, como trivialmente $(N-M) \cap M=\emptyset$, existe ponto $p_{i}$ tal que $\operatorname{supp} \sigma_{i} \subset U_{p_{i}}$. Então construímos a seção suave em $N$

$$
\zeta=\sum_{i} \sigma_{i} \bar{\zeta}_{p_{i}}
$$

tal que $\left.\zeta\right|_{M}=\Psi$

Utilizando o fato que $\zeta$ é suave, pela propriedade Cont. acima, ao redor de cada ponto $p$ de $M$, onde sabemos que $\zeta$ é transversal a $Z$, existirá um aberto de $N, V_{p}$, onde $\zeta$ permanece transversal a todos os $Q^{j}$. A coleção $\left\{U_{p} \cap V_{p} \mid p \in M\right\} \cup(N-M)$ ainda é cobertura aberta de $N$. Agora tomando qualquer cobertura de $N-M$ por domínios de trivializações podemos proceder exatamente como na construção de $\Psi$, sendo que sobre $M$, i.e.: $\left.\zeta\right|_{M}$ estará a salvo das perturbações necessárias para deixá-la transversal a $Z$ em $N-M$.

Finalmente chegamos ao Teorema de Classificação de Fibrados

Theorem 28 Qualquer que seja $E, G$-fibrado vetorial sobre $M, E \simeq f_{0}^{*} \xi_{G}$ para alguma função suave $f_{0}: M \rightarrow B_{G}$. Se além disso $f_{1}^{*} \xi_{G} \simeq E$, então $f_{0}$ e $f_{1}$ são homotópicas .

Dem: Nós provaremos para o caso $G=\mathbf{G L}(k)$. Tomamos $B_{G}$ como sendo a veriedade Grassmaniana $G(k, l)$, i.e.: os pontos de $B_{G}$ são os subespaços $k$-dimensionais de $\mathbb{R}^{l}$. Definimos ainda

$$
\xi_{G}=\left\{(p, v) \in G(k, l) \times \mathbb{R}^{l} \mid v \in p\right\}
$$


o que significa que a fibra sobre o ponto $p$ da variedade base $G(k, l)$ ć justamente o subespaço de $\mathbb{R}^{l}$ correspondente a $p$. Agora, pela proposição anterior, nós sabemos que existe um isomorfismo fo de $E$ com uma $k$-subfibrado do $M \times \mathbb{R}^{l}$. Portanto nós definimos um mapa

$$
\begin{aligned}
f_{0}: & M \rightarrow G(k, l) \\
& x \mapsto \varphi_{0}\left(\pi_{E}^{-1}(x)\right)
\end{aligned}
$$

Lembramos que como $\varphi_{0}$ ć injcção lincar (tom posto máximo $k<l$ ), ou sc̣ja, ć isomorfismo linear sobre a imagem. Logo nós temos que

$$
\begin{gathered}
f_{0}^{*} \xi_{G}=\left\{(x, v) \in M \times \xi_{G} \mid v \in f_{0}(x)\right\} \simeq\left\{(x, v) \in M \times \xi_{G} \mid v \in \varphi_{0}\left(\pi_{E}^{-1}(x)\right)\right\} \\
\therefore \quad f_{0}^{*} \xi_{G} \simeq\left\{(x, v) \mid x \in M \text { e } v \in \pi_{E}^{-1}(x)\right\}=E
\end{gathered}
$$

Agora suponhamos que $\varphi_{1}: E \rightarrow M \times \mathbb{R}^{l}$ seja tal que temos

$$
f_{1}^{*} \xi_{G}=\left\{(x, v) \in M \times \xi_{G} \mid v \in \varphi_{1}\left(\pi_{F}^{-1}(x)\right)\right\} \simeq F
$$

Tomamos a subvariedade $(\Lambda \times\{0\}) \cup(M \times\{1\}) \subset M \times I$. Então pela extensão que construímos na Prop.25, e utilizando a Prop.19, temos então um isomorfismo $\varphi$ entre $E \times I$ e um subfibrado de $\mathbb{R}_{M \times I}^{l}$ tal que $\left.\varphi\right|_{M \times\{0\}}=\varphi_{0}$ e $\left.\varphi\right|_{M \times\{1\}}=\varphi_{1}$. É claro então que

$$
\begin{aligned}
F: & M \times I \rightarrow G(l, k) \\
& (x, t) \mapsto \varphi\left(\pi_{E \times I}^{-1}(x, t)\right)
\end{aligned}
$$

é uma homotopia entre $\int_{0}$ e $\int_{1}$. Ou seja, além de existência estabelecemos unicidade (em classe de homotopia)

\section{Classes e Números Característicos}

Classes e números característicos são justamente os invariantes globais que associam à cada fibrado vetorial uma classe de cohomologia da variedade base.

Dado $f: N \rightarrow M$, nós obtemos os mapas induzidos

$$
\begin{array}{ll}
f_{\text {vect }}^{*}: & \operatorname{Vect}_{G}(M) \rightarrow \operatorname{Vect}_{G}(N) \quad \text { e } \\
f^{*}: & H^{*}(M) \rightarrow H^{*}(N)
\end{array}
$$

onde $H^{*}(M)$ é o anel de cohomologia de Rham de $M$.

Assumindo que $f^{*}$ também só depende da classe de homotopia, nós temos que ambos os mapas só dependem da classe de homotopia de $f$. Uma classe característica $c$ (para G-fibrados) associa a $E$ uma classe de cohomologia $c(E)$ em $H^{*}(M)$ de uma forma natural.

Definition 21 Uma classe característica $C$ para G-fibrados é um mapa ${ }^{6}$ :

$$
C: \operatorname{Vect}_{G} \rightarrow H^{*}
$$

tal que dado um G-fibrado vetorial sobre a variedade suave $M$ e um mapa suave $f: N \rightarrow M$, onde $N$ é também suave, então

$$
f^{*}(c(E))=c\left(f_{\text {vect }}^{*}(E)\right)
$$

\footnotetext{
${ }^{6} \mathrm{Na}$ linguagem de teoria das categorias, $C$ é uma transformação natural do funtor Vect $G$ ao funtor $H^{*}$, ambos considerados como funtores contravariantes na categoria de variedades suaves.
} 
O sub-índice "vect" é na verdade desnecessário, e o suprimiremos de agora em diante. Denotaremos por Char $(G)$ o conjunto das classes características de G-fibrados.

Proposition 26 Seja $\xi_{G}$ o $G$-fibrado universal sobre o espaço $B_{G}$, então

$$
c \mapsto c\left(\xi_{G}\right)
$$

é isomorfismo entre Char $(G)$ e $H^{*}\left(B_{G}\right)$.

Dem: Seja $\sigma \in H^{*}\left(B_{G}\right)$, definimos

$$
\begin{aligned}
c: H^{*}\left(B_{G}\right) & \rightarrow \operatorname{Char}(G) \\
\sigma & \mapsto c_{\sigma}
\end{aligned}
$$

onde

$$
\begin{aligned}
& c_{\sigma}: \operatorname{Vect}_{G} \rightarrow H^{*} \\
& E=f_{E}^{*} \xi_{G} \mapsto f_{E}^{*}(\sigma)
\end{aligned}
$$

onde agora $c_{\sigma}$ é um funtorial de Vect ${ }_{G}$ em $H^{*}$ que leva $E$ em $f_{E}^{*}(\sigma)$, onde $f_{E}: M \rightarrow B_{G}$ é tal que $f_{E}^{*}\left(\xi_{G}\right)=E \in \operatorname{Vect}_{G} M$, logo existe um único (a menos de classes de homotopia) tal $f_{E}$ pelo Teo.28.

Resumidamente poderíamos definir

$$
\begin{aligned}
c_{E}: H^{*} B_{G} & \rightarrow H^{*} M \\
\sigma & \mapsto f_{E}^{*} \sigma
\end{aligned}
$$

Isto é, $c_{E}=f_{E}^{*}$, ou seja $c_{E}(\sigma)=c_{\sigma}(E)$. Nós temos então que $c_{E}=c_{F}$ se $f_{E}$ é homotópica a $f_{F}$, ou seja, se $F \simeq E$. Logo, $c_{\sigma}$ está bem definido.

Antes de mais nada precisamos mostrar que $c_{\sigma}$ é uma transformação natural de Vect $_{G}$ em $H^{*}$. Isto é, se $g: N \rightarrow M$ é liso, então temos:

$$
c_{\sigma}\left(g^{*}(E)\right)=c_{\sigma}\left(g^{*} f_{E}^{*} \xi_{G}\right)=c_{\sigma}\left(\left(f_{E} \circ g\right)^{*} \xi_{G}\right):=\left(f_{E} \circ g\right)^{*}(\sigma)=g^{*} f_{E}^{*}(\sigma)=g^{*} c_{\sigma}(E)
$$

Colocando de outra forma $f_{g^{*}(E)}(\sigma)=g^{*} f_{E}$. Provamos, então, que para todo $\sigma \in B_{G}^{*}$ existe $c_{\sigma} \in$ Char $G$, tal que $c_{\sigma}\left(\xi_{G}\right)=\sigma$, ou seja, $c \mapsto \xi_{G}$ é sobrejeção de $\operatorname{Char}(G)$ em $H^{*} B_{G}$.

Além disso, sejam $c_{1}, c_{2} \in \operatorname{Char}(G)$ tal que $c_{1}\left(\xi_{G}\right)=c_{2}\left(\xi_{G}\right) \in H^{*} B_{G}$ Então

$$
f^{*} c_{1}\left(\xi_{G}\right)=f^{*} c_{2}\left(\xi_{G}\right)=c_{1}\left(f^{*} \xi_{G}\right)=c_{2}\left(f^{*} \xi_{G}\right)
$$

Portanto, como qualquer que seja $E \in$ Vect $_{G} M$ existe $f: M \rightarrow B_{q}$ tal que $f^{*} \xi_{G}=E$ obtemos que $c_{1}(E)=c_{2}(E)$ para todo $E \in \operatorname{Vect}_{G}$. Ou seja, $c_{1}=c_{2}$.

Apesar de ter grande importância conceitual e de ser indiscutivelmente uma bela construção teórica, esta descrição de classes características não nos fornece uma maneira prática de calcular classes características.

Números característicos são definidos da seguinte forma: se $\lambda \in H^{m}(M)$, com $M$ fechado, então os números

$$
\int_{M} c(E) \quad \text { onde } \quad c \in \operatorname{Char}(G)
$$

são chamados de números característicos de um $G$-fibrado sobre $M$. Eles são claramente invariantes (por classes de isomorfismo de fibrados), portanto, como um fibrado trivial é induzido por um mapa constante, todas suas classes características e números característicos são nulos, o que nos fornece uma boa maneira de testar não trivialidade. Estudemos agora um caso particular de classes características. 


\section{Classes de Chern}

As classes de Chern fornecem um teste siples de verificar se dois fibrados vetoriais sobre a mesma base não são isomorfos (nada garante que se tiverem a mesma forma de Chern serão isomorfos). É muitas vezes importante " contar" quantas seções linearmente independentes um fibrado vetorial possuí, as classes de Chern fornecem informações sobre isso através do Teorema de Riemann-Roch e o do Teorema do Índice de Atiyah-Singer.

A classe de Chern de um fibrado em $M$, pelo Teorema de Classificação de Fibrados Vetoriais, pode ser dada pelo pull-back das classes de Chern no fibrado universal (que podem por sua vez serem descritas explicitamente por ciclos de Schubert [21]), mas não será essa a nossa abordagem.

De qualquer forma, o significado intuitivo das classes de Chern (e outras classes características) concerne os "zeros necessários" de uma seção (não nula) de um fibrado vetorial, ou obstruções para a construção de certas seções ${ }^{7}$. Daremos a seguir um tratamento informal e pouco algébrico de formas de Chern, em acordo com o resto da seção. Para um tratamento mais formalizado indicamos [9].

Uma propriedade interessante do operador tr que necessitaremos agora, é que se $\lambda$ é uma p-forma a valores em $\operatorname{End}(E)$, utilizando a derivada covariante exterior $D$, por (1.117):

$$
D \lambda=d \lambda+\omega \wedge \lambda-(-1)^{p} \lambda \wedge \omega
$$

portanto em coordenadas temos $\lambda=\lambda_{j}^{i} e_{i} \otimes e^{j}$ onde $\lambda_{j}^{i}$ é uma $p$-forma,

$$
D \lambda=d \lambda_{j}^{i} e^{j} \otimes e_{i}+\omega_{i}^{k} \wedge \lambda_{j}^{i} e^{j} \otimes e_{k}-(-1)^{p} \lambda_{i}^{j} \wedge \omega_{k}^{i} e^{k} \otimes e_{j}
$$

e portanto

$$
\operatorname{tr}(D \lambda)=d(\operatorname{tr} \lambda)+\omega_{i}^{j} \wedge \lambda_{j}^{i}-(-1)^{p} \lambda_{i}^{j} \wedge \omega_{j}^{i}=d(\operatorname{tr} \lambda)
$$

Voltamos ao fato que o funcional de Yang-Mills

$$
Y M(D):=\left\langle\left\langle\Omega_{D}, \Omega_{D}\right\rangle\right\rangle=\frac{1}{2} \int_{M} \operatorname{tr}\left(\Omega_{D} \wedge * \Omega_{D}\right)
$$

só depende da mótrica através de *. Nós poderíamos tentar escrever uma ação que não envolve a métrica, por exemplo em 4D:

$$
S_{4}(D)=\int_{M} \operatorname{tr}(\Omega \wedge \Omega)
$$

É claro que se $\Omega=\Omega_{+}$então $* \Omega=\Omega$ e nós temos que então $Y M(D)=S_{4}(D)$. É interessante notar que nossa nova ação está intimamente relacionada com dualidade e com a teoria de Yang-Mills.

Comecemos calculando os pontos críticos de $S_{4}$. Seja $\tilde{D}=D+t \gamma$ onde $\gamma \in \Gamma\left(\Lambda^{1}(T M) \otimes\right.$ $\operatorname{Ad}(E)$ ). Então, como sabemos $\Omega_{D+t \gamma}=\Omega_{D}+t D \gamma+t^{2} \gamma \wedge \gamma$, portanto

$$
\begin{aligned}
& \left.\frac{d}{d t}\right|_{t=0} \Omega_{D+t \gamma} \wedge \Omega_{D+t \gamma}=2\left(\Omega_{D} \wedge D \gamma\right) \\
& \therefore \delta S_{4}(D)=2 \int_{M} \operatorname{tr}\left(\Omega_{D} \wedge D \gamma\right)=2 \int_{M} \operatorname{tr}\left(D \Omega_{D} \wedge \gamma\right)=0
\end{aligned}
$$

\footnotetext{
${ }^{7} \mathrm{Um}$ exemplo de tais obstruções que podem surgir é dado pelo teorema da "bola peluda", que nos diz ser impossível construir um campo vetorial global não nul o sobre $S^{2}$.
} 
Onde usamos a identidade de Bianchi na última igualdade e na penúltima

$$
\begin{gathered}
D \gamma \wedge \Omega_{D}=D \gamma \wedge *\left(* \Omega_{D}\right)=\left\langle D \gamma, * \Omega_{D}\right\rangle \\
\therefore \quad\left\langle\left\langle D \gamma, * \Omega_{D}\right\rangle\right\rangle=\left\langle\left\langle\gamma, * D *\left(* \Omega_{D}\right)\right\rangle\right\rangle=\left\langle\left\langle\gamma, * D \Omega_{D}\right\rangle\right\rangle=\int_{M} \operatorname{tr}\left(D \Omega_{D} \wedge \gamma\right)
\end{gathered}
$$

Outra forma de obter este resultado ${ }^{8}$ é utilizar Bianchi

$$
D \gamma \wedge \Omega_{D}=D\left(\gamma \wedge \Omega_{D}\right)+\gamma \wedge D \Omega_{D}=D\left(\gamma \wedge \Omega_{D}\right)
$$

e (3.15) juntamente com Stokes:

$$
2 \int_{M} \operatorname{tr}\left(\Omega_{D} \wedge D \gamma\right)=2 \int_{M} \operatorname{tr}\left(D\left(\gamma \wedge \Omega_{D}\right)\right)=2 \int_{M} d \operatorname{tr}\left(\gamma \wedge \Omega_{D}\right)=0
$$

Ora, mas nós obtemos então que qualquer conexão $D$ é ponto crítico de $S_{4}$ !

Ainda assim, vamos prosseguir mais um pouco nessa linha e generalizar:

$$
S_{2 n}(D)=\int_{M} \operatorname{tr} \Omega^{n}
$$

onde $M^{2 n}$ e $\Omega^{n}=\underbrace{\Omega \wedge \cdots \wedge \Omega}_{n}$. Tomando a variação, obtemos, empregando (3.15):

$$
\begin{aligned}
\left.\frac{d}{d t}\right|_{t=0} \Omega_{D+t \gamma}^{n} & =n\left(\Omega_{D}^{n-1} \wedge D \gamma\right)=n D\left(\Omega^{n-1} \wedge \gamma\right) \\
\left.\therefore \operatorname{tr} \frac{d}{d t}\right|_{t=0} \Omega_{D+t \gamma}^{n} & =n d\left(\operatorname{tr}\left(\Omega_{D}^{n-1} \wedge \gamma\right)\right)
\end{aligned}
$$

Logo:

$$
\delta S_{2 n}(D)=\int_{M} \operatorname{tr}\left(\left.\frac{d}{d t}\right|_{t=0} \Omega_{D+t \gamma}^{n}\right)=n \int_{M} \operatorname{tr}\left(\Omega_{D}^{n-1} \wedge D \gamma\right)=n \int_{M} d \operatorname{tr}\left(\Omega_{D}^{n-1} \wedge \gamma\right)=0
$$

Chamamos $\operatorname{tr} \Omega^{n}$ a $n$-ésima forma de Chern.

Ainda por (3.15), obtivemos que $d\left(\operatorname{tr} \Omega_{D}^{n}\right)=\operatorname{tr}\left(D \Omega_{D}^{n}\right)=0$. Portanto as formas de Chern são fechadas. Isso significa que a $k$-ésima forma de Chern define uma classe de cohomologia, em $H^{2 k}(M)$. Agora, a forma de Chern realmente depende da conexão $\omega$, mas sua classe de cohomologia não, isto é, se mudarmos a conexão $\omega$, a forma de Chern se desloca por uma forma exata. Para ver esse resultado, tomemos $\omega^{\prime}=\omega+\gamma$ e $\omega_{s}=\omega+s \gamma$, então nós temos (utilizando (3.20)):

$$
\operatorname{tr}\left(\Omega^{\prime k}\right)-\operatorname{tr}\left(\Omega^{k}\right)=\int_{0}^{1} \frac{d}{d s} \operatorname{tr}\left(\Omega_{s}^{k}\right) d s=k \int_{0}^{1} d \operatorname{tr}\left(\gamma \wedge \Omega_{s}^{k-1}\right) d s=k d \int_{0}^{1} \operatorname{tr}\left(\gamma \wedge \Omega_{s}^{k-1}\right) d s
$$

que é, portanto, exata.

\footnotetext{
${ }^{8}$ Lembramos que $M$ é uma variedade sem bordo.
} 
Utilizando o Teo. 26, outra forma de percebcrmos que a forma de Chern só depende da classe de homotopia de $M$, isto é, que duas formas de Chern diferem por uma forma exata (estão na mesma classe de cohomologia) (Milnor):

Dadas duas conexões $\nabla^{0}$ e $\nabla^{1}$ em $E$, nós usamos $\mathrm{pr}_{1}: \Lambda \times I \rightarrow M$ para trazê-las para

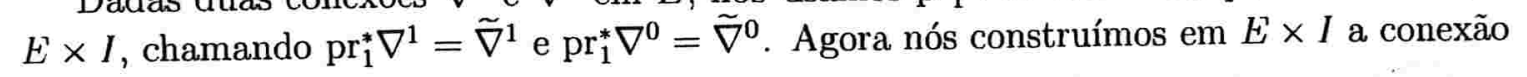

$$
\widetilde{\nabla}=\left(\mathrm{pr}_{2}\right)\left(\widetilde{\nabla}^{1}\right)+\left(1-\mathrm{pr}_{2}\right)\left(\widetilde{\nabla}^{0}\right)
$$

onde a função $\operatorname{pr}_{2}: M \times I \rightarrow \mathbb{R}$ é a projeção $M \times I \rightarrow I \subset \mathbb{R}$. Agora seja

$$
\begin{aligned}
\varepsilon_{t}: & M \rightarrow M \times I \\
& x \mapsto(x, l)
\end{aligned}
$$

Então $\varepsilon_{t}^{*}(E \times I)=E$ e portanto $\varepsilon_{0}^{*} \widetilde{\nabla}=\nabla^{0}$ e $\varepsilon_{1}^{*} \widetilde{\nabla}=\nabla^{1}$.

$$
\begin{aligned}
& \therefore \quad \operatorname{tr}\left(\Omega_{\iota}\right)=\operatorname{tr}\left(\varepsilon_{t}^{*} \Omega_{\tilde{D}}\right)=\varepsilon_{t}^{*} \operatorname{tr}\left(\Omega_{\tilde{D}}\right) \quad \text { então } \\
& \operatorname{tr}\left(\Omega_{0}\right)=\varepsilon_{0}^{*}\left(\operatorname{tr}\left(\Omega_{\tilde{D}}\right)\right) \quad \text { e } \operatorname{tr}\left(\Omega_{1}\right)=\varepsilon_{1}^{*} \operatorname{tr}\left(\Omega_{\tilde{D}}\right)
\end{aligned}
$$

Onde utilizamos que $f^{*} \operatorname{tr}(\lambda)=\operatorname{tr}\left(f^{*} \lambda\right)$, resultado válido para qualquer $\lambda p$-forma a valores em $\operatorname{End}(E)$. Isto pode ser visto lembrando que se $\left\{c_{i}\right\}$ ć basc do $E_{f(x)}$, então $\left\{f^{*} c_{i}\right\}=\left\{c_{i}\right\}$ é base de $\left(f^{*} E\right) x$, agora escrevendo escrevendo em coordenadas o resultado é trivial.

Logo, obtcmos $\operatorname{tr}\left(\Omega_{1}\right)$ e $\operatorname{tr}\left(\Omega_{0}\right)$ são pull-backs da mesma forma fechada $\operatorname{tr}\left(\Omega_{\tilde{D}}\right)$ por dois mapas diferentes mas homotópicos, $\operatorname{logo} \varepsilon_{0}^{*}\left(\Omega_{\tilde{D}}\right) \simeq \varepsilon_{1}^{*}\left(\Omega_{\tilde{D}}\right)$ isto é, são co-homólogas.

Logo podemos definir a $k$-ésima classe de Chern $c_{k}(F)$ do fibrado vetorial $F$, sobre $M$ como a classe de cohomologia de $\operatorname{tr}\left(\Omega^{k}\right)$, onde $\Omega$ é a forma de curvatura de qualquer forma de curvatura em $F$. Estes invariantes são ferramentas importantes na classificação de fibrados vetoriais. Utilizando uma definição de caráter mais topológico para as formas de Chern é possível mostrar que, quando apropriadamente normalizados, suas integrais sobre uma variedade compacta orientada $\Lambda$ são números inteiros. A normalização requerida é

$$
c_{k}(E)=\frac{(i / 2 \pi)^{k}}{n !} \int_{M} \operatorname{tr}\left(\Omega^{k}\right)
$$

A integralidade destas classes são de extrema importância na Teoria de Chern-Simons. Mostraremos uma aplicação destas classes aos monopólos magnéticos, quando estudarmos o eletromagentismo como teoria de gauge. Estritamente falando, classes de Chern se aplicam à fibrados vetoriais complexos, sendo seu análogo para fibrados reais as classes de Pontryagin, mas não adentraremos nesse ponto aqui. $\mathrm{Na}$ verdade os fibrados que realmente nos interessam, devido à quantização (a função de onda ter valores complexos), são os complexos. 


\section{Chapter 4}

\section{Aplicações}

Os maiores matemáticos, como Archimedes, Newton, e Gauss, sempre uniram teoria e aplicações em igual medida - Felix Klein.

\subsection{Eletromagnetismo}

As equações de Maxwell tiveram maior impacto sobre a história humana do que quaisquer dez presidentes americanos. - Carl Sagan

E a continuidade de nossa ciência não foi afetada por todos estes turbulentos acontecimentos, assim como as teorias antigas sempre são inclusas como casos limítrofes nas novas. - Max Born, referindo-se à relatividade restrita e à mecânica quântica.

\section{Equações de Maxwell}

Poderíamos adicionar à citação de Born que não só são as teorias antigas inclusas nas novas teorias, sendo a ciência uma forma progressiva de conhecimento, mas que são elas muitas vezes a pedra fundamental na compreensão da teoria mais evoluída, os ombros dos gigantes sobre os quais todos subimos ${ }^{1}$. A teoria eletromagnética de Maxwell por exemplo serviu como inspiração tanto para a relatividade geral quanto para a teoria de gauge. Nesta seção, partindo das equações de Maxwell, construíremos uma teoria de gauge em um U(1)-fibrado sobre a variedade 4-dimensional $M$.

As equações de Maxwell descrevem o comportamento de dois campos vetoriais, o campo elétrico $\vec{E}$ e o magnético $\vec{B}$. Estes campos são funções do tempo, que é um parâmetro real $t$, e são definidos sobre todo o espaço que (em sua forma usual) é tido como $\mathbb{R}^{3}$. Os campos dependem ainda da densidade de carga elétrica $\rho$ e da densidade de corrente $\vec{\jmath}$, que é um campo vetorial dependente do tempo em $R^{3}$.

As quatro equações, na notação vetorial devida a Hertz (forma em que é mais conhecida),

\footnotetext{
1 "Se eu enxerguei mais longe, foi porque me apoiei em ombros de gigantes." Isaac Newton.
} 
em unidades apropriadas para que a velocidade da luz seja $c=1$ são:

$$
\begin{aligned}
\nabla \cdot \vec{B} & =0 \\
\nabla \times \vec{E}+\frac{\partial \vec{B}}{\partial t} & =0 \\
\nabla \cdot \vec{E} & =\rho \\
\nabla \times \vec{B}-\frac{\partial \vec{E}}{\partial t} & =\vec{\jmath}
\end{aligned}
$$

A semelhança entre as equações (4.1), (4.2) às equações (4.3), (4.4) respectivamente, é intrigante. No entanto há uma disparidade: o operador divergente em $\mathbb{R}^{3}$ é associado a um operador diferencial de primeira ordem sobre 2-formas, enquanto o operador rotacional atua sobre 1-formas, mas queremos aplicar ambos sobre $\vec{E}$ e $\vec{B}$. Com um auxílio do operador estrela de Hodge podemos transformar um no outro, quem sabe estabelecendo uma relação entre o primeiro par de equações e o segundo.

Na relatividade, $M$ é uma variedade suave lorentziana, i.e.: equipada com um produto bilinear simétrico não-degenerado, mas que não é positivo definido, tem assinatura $(3,1)$. Para facilitar a notação, vamos considerar o caso de uma métrica de Minkowski ${ }^{2}$, que chameremos de $\eta$.

Comecemos analisando as equações (4.1) e (4.2). Para escrever o campo elétrico como uma 1-forma, precisamos compatibilizar grandezas vetoriais, usualmente utilizadas no eletromagnetismo, com a descrição em formas que estamos perseguindo. Para isso basta usar o operador $\sharp$ (definido na Sec.1.3). No entanto, como estamos na métrica de Minkowski, isso simplesmente significa fazermos $\partial_{i}^{\sharp}=d x^{i}$. E portanto tomamos $E=\vec{E}^{\sharp}$. Para o campo magnético, que queremos encarar como uma 2-forma ${ }^{3}$, faremos $B=*_{S}\left(\vec{B}^{\sharp}\right)$, onde $*_{S}$ é o operador de Hodge restrito ao subespaço puramente espacial (com a métrica euclidiana). É fácil ver que

$$
(\nabla \times \vec{v})^{\sharp}=-*_{S} d_{S}(\vec{v})^{\sharp} \text { e }(\nabla \cdot \vec{v})^{\sharp}=*_{S}\left(d_{S}\left(*_{S}(\vec{v})^{\sharp}\right)\right)
$$

A forma natural de produzirmos uma 2-forma a partir de $E$ (sem utilizar *) seria tomar $E \wedge d t$, e agora unimos os dois em uma única 2-forma chamada de campo eletromagnético:

$$
F=B+E \wedge d t=*_{S}\left(\vec{B}^{\sharp}\right)+(\vec{E})^{\sharp} \wedge d t
$$

E agora, separando a diferencial exterior em sua parte espacial (que opera com diferenciação só nos índices espaciais) e sua parte temporal, i.e.: $d=d_{S}+d_{\iota}$ temos

$$
\begin{aligned}
d F & =d B+d E \wedge d t \\
& =d_{S} B+\partial_{\iota} B \wedge d t+\left(d_{S} E+\partial_{\iota} E \wedge d t\right) \wedge d t \\
& =d_{S} B+\left(\partial_{t} B+d_{S} E\right) \wedge d t
\end{aligned}
$$

\footnotetext{
${ }^{2} \mathrm{Na}$ verdade, o Princípio de Equivalência de Einstein afirma que localmente um referencial inercial é equivelente ao espaço de Minkowski, o que seria o análogo de tomarmos um gauge quase-canônico em TM Sec.1.6.

${ }^{3}$ Poderíamos colocar os dois campos como 1-formas, deixando o opererador * cuidar da ordem necessária. à aplicação da derivada exterior adequada, ao prosseguir por essa linha no entanto não encontramos equações de Yang-Mills, e as equações se tornam menos elegantes.
} 
Notemos que o primeiro termo da última expressão não contém $d t$, logo os dois termos são linearmente independentes, portanto a equação $d F=0$ equivale a:

$$
\begin{aligned}
d_{S} B & =0 \\
\partial_{t} B+d_{S} E & =0
\end{aligned}
$$

E obtemos de (4.8) e 4.9), em uma forma comparável às equações (4.1) e (4.2):

$$
\begin{aligned}
& (\nabla \cdot \vec{B}) \operatorname{vol}_{S}=0 \\
& *_{S}\left(\partial_{t} \vec{B}\right)^{\sharp}+*_{S}(\nabla \times \vec{E})^{\sharp}=*_{S}\left(\left(\partial_{t} \vec{B}+\nabla \times \vec{E}\right)^{\sharp}\right)=0
\end{aligned}
$$

Portanto claramente equivalem a (4.1) e (4.2). Uma vantagem da linguagem de formas diferenciais é sua generalidade. Podemos tomar nosso espaço-tempo como sendo qualquer variedade $M$, definindo o campo eletromagnético como uma 2-forma $F$ em $M$, as primeiras equações de Maxwell dizem simplesmente que

$$
d F=0
$$

A nossa divisão de espaço-tempo em espaço $e$ tempo é que foi de alguma forma arbitrária, já que raramente podemos tomar $M=S \times \mathbb{R}$, onde $S$ é uma variedade riemanniana de três dimensões. Somente quando fizermos uma tal separação (localmente) é que podemos falar de campo eláetrico e campo magnético, separadamente.

Utilizando a não degenerescência da métrica de Minkowski podemos definir o operador * de Hodge normalmente (lembramos que definimos por (1.38) uma métrica em $\Lambda^{p}(V)$ dada uma métrica em $V$ ). Não é difícil mostrar que se $M$ for uma variedade semi-riemanniana de dimensão $n$ e assinatura $(s, n-s)$ nós temos $*_{n-p} *_{p}=(-1)^{p(n-p)+s}$. É relativamente fácil perceber que $*\left(*_{s}\left(\vec{v}^{\sharp}\right)\right)=\vec{v}^{\sharp} \wedge d t$, já que, se o $*_{s}$ leva $\vec{v}^{\sharp}$ em formas a ele ortogonais espacialmente, o operador * seleciona as duas direções ortogonais a esses dois vetores, uma é a direção temporal, logo a outra é no sentido inicial do vetor, restando checar os sinais apropriados (orientação), o que deixaremos a cargo do leitor. Da mesma maneira é fácil verificar que $*\left(\vec{v}^{\sharp} \wedge d t\right)=*_{S}\left(\vec{v}^{\sharp}\right)$. Obtemos então:

$$
\begin{aligned}
& *(E \wedge d t)=*_{S}\left(\vec{E}^{\sharp}\right) \\
& * B=*\left(*_{S}\left(\vec{B}^{\sharp}\right)\right)=(\vec{B})^{\sharp} \wedge d t \\
\therefore \quad & * F=-*_{S}\left(\vec{E}^{\sharp}\right)+(\vec{B})^{\sharp} \wedge d t
\end{aligned}
$$

Ou seja, fizemos a mudança

$$
\vec{E} \rightarrow-\vec{B} \text { e } \vec{B} \rightarrow \vec{E}
$$

Que é uma (de duas) das diferenças entre as equações (4.1), (4.2) às (4.3) e (4.4). Escrevemos (4.7) na forma

$$
d F=d_{S} B+\left(\partial_{t} B+d_{S} E\right) \wedge d t=d_{S} *_{S}\left(\vec{B}^{\sharp}\right)+\left(*_{S} \partial_{t}\left(\vec{B}^{\sharp}\right)+d_{s}\left(\vec{E}^{\sharp}\right)\right) \wedge d t
$$

Logo podemos escrever (utilizando que $*(A \wedge d t)=*_{S} A$, e $\left(*_{S}\right)_{2} \circ\left(*_{S}\right)_{1}=-1$ na passagem da segunda para a terceira linha e $*_{1} *_{3}=1$ na passagem da primeira para a segunda):

$$
\begin{aligned}
d * F & =d_{S} *_{S}\left(\vec{E}^{\sharp}\right)+\left(*_{S} \partial_{t}\left(\vec{E}^{\sharp}\right)-d_{s}\left(\vec{B}^{\sharp}\right)\right) \wedge d t \\
& \left.=(\nabla \cdot \vec{E}) \operatorname{vol}_{S}+*\left(*\left(*_{S} \partial_{t}\left(\vec{E}^{\sharp}\right)-d_{s}\left(\vec{B}^{\sharp}\right)\right) \wedge d t\right)\right) \\
& \left.=*\left((\nabla \cdot \vec{E}) d t-\partial_{t}\left(\vec{E}^{\sharp}\right)-*_{S} d_{s}\left(\vec{B}^{\sharp}\right)\right)\right)
\end{aligned}
$$


E obtemos então que:

$$
\left.* d * F=(\nabla \cdot \vec{E}) d t-\partial_{\iota}\left(\vec{E}^{\sharp}\right)-*_{s} d_{s}\left(\vec{B}^{\sharp}\right)\right)
$$

A outra diferença entre os dois pares de equações, é que as últimas contém termos não homogêneos, a carga e a corrente. Mas carga é simplesmente a corrente que está, em relação ao nosso referencial, parada, i.e.: é um vetor sem projeção espacial, cuja única componente não nula é a temporal. Escrevemos pois, tomando coordenadas $\left\{x_{\mu}\right\}_{\mu=0}^{3}$ :

$$
\vec{J}=\rho \partial_{0}+j^{1} \partial_{1}+j^{2} \partial_{2}+j^{3} \partial_{3}
$$

E assim unimos a carga e a corrente em uma ínica 1-forma

$$
J=-\rho d x^{0}+j_{i} d x^{i}
$$

Agora, simplesmente aplicando b em (4.13), e lembrando que $\left(d x^{0}\right)^{b}=-\partial_{0}$, chegamos ao outro par das equações:

$$
* d * F=J
$$

Historicamente, foi Faraday que descobriu, em 1831, que um campo magnético variando no tempo induziria um rotacional não-nulo no campo eletrico (4.2). Em 1861 Maxwell percebeu que a lei de conservação de cargas (elétricas) poderia ser introduzida automaticamente na Lei de Biot-Savart:

$$
\nabla \times \vec{B}=\vec{\jmath}
$$

Simplesmente adicionando-se a ela um termo $-\frac{\partial \vec{E}}{\partial t}=\vec{\jmath}$ e tomando o divergente chegava-se à cquação de continuidade

$$
\frac{d \rho}{d t}=\nabla \cdot \vec{\jmath}
$$

É interessante notar que Einstein fez uma generalização muito parecida para chegar à sua equação:

$$
R_{\mu \nu}=\kappa T_{\mu \nu} \rightarrow R_{\mu \nu}-\frac{1}{2} g_{\mu \nu} R=\kappa T_{\mu \nu}
$$

que dessa forma automaticamente incorporava a conservação infinitesimal ${ }^{4}$ do tensor de energia-momento através da identidade de Bianchi contraída ${ }^{5}$ que na notação de " índices abstratos" [10]é :

$$
\nabla^{c}\left(R_{c \cdot d}-\frac{1}{2} g_{c \cdot d} R\right)=0
$$

Na verdade essa similaridade têm uma raíz comum, a identidade de Bianchi (1.72). Isso pode ser visto no nosso caso escrevendo-se a (4.15) na forma

$$
d * J=0
$$

\footnotetext{
${ }^{4}$ Ver [5] Cap.19, ou [1.5] Cap.4 para uma explicação das dificuldades de passarmos de uma equação de conservação infinitesimal para. uma. lei de conservação de carga e momento.

${ }^{5}$ Havia ainda o agravante de que sem esse termo, como se pode notar pela identidade abaixo, que a curvatura escalar é uma constante .
} 
agora, simplesmente aplicando $d *$ em ambos os lados da equação (4.14) obtemos o resultado. A disparidade entre as duas equações para $F$ pode ser atribuída a inexistência (pelo que até hoje se sabe) de monopólos magnéticos. Qualquer semelhança com as equações de Yang-Mills não é mera coincidência.

Utilizando o Lema de Poincaré, pela equação $d F=0$, se $U$ for uma região contrátil ${ }^{6}$ existe uma 1-forma $A$ sobre $U$ tal que $\left.F\right|_{U}=d A$. Logo a segunda equação fica

$$
* d * d A=0
$$

Claramente, qualquer transformação $A \rightarrow A+d f$ onde $f: U \rightarrow \mathbb{R}$ não afeta as equações. Por outro lado, suponhamos que nós tenhamos um U(1)-fibrado vetorial, $E$. Para simplificar, assumimos que $E=M \times \mathbb{C}$. Pelo Teo.8 uma conexão $\nabla$ em $E$ pode ser então descrita por uma forma de conexão, que aqui chamaremos de potencial vetor, que é uma 1-forma a valores em $\operatorname{End}(E)$. Mas como $\operatorname{End}(\mathbb{C}) \simeq \mathbb{C}$ canonicamente, isto equivale a dizer que temos uma forma a valores complexos. Tomamos a representação fundamental de $\mathbf{U}(1)$ sobre $\mathbb{C}$. A álgebra de Lie de U(1) é dada por

$$
\mathfrak{u}(1)=\{i x \mid x \in \mathbb{R}\}
$$

$\mathrm{O}$ que significa que as componentes de $A$ são funções puramente imaginárias, o que pode causar certo disconforto, mas que em todo caso pode ser consertado meramente estipulando que essa conexão seja $i$ vezes a conexão real. Agora suponhamos que apliquemos uma transformação de gauge a este potencial vetor. Como $E$ é trivial nós podemos pensar na transformação de gauge como uma função $g: M \rightarrow \mathrm{U}(1)$. E obtemos então, lembrando que $\mathrm{U}(1)$ é abeliano:

$$
\widetilde{A}=g A g^{-1}+g d g^{-1}=A+g d g^{-1}
$$

logo se pudermos escrever $g=e^{-f}$ para alguma função a valores imaginários $f$, nós recuperamos

$$
\widetilde{A}=A+d f
$$

Claramente agora a curvatura é simplesmente o campo eletromagnético e as equações de Maxwell no vácuo são as equações de Yang-Mills para $G=\mathrm{U}(1)$. Lembramos que se o grupo não for abeliano, a própria derivada exterior covariante envolve termos do potencial , deixando as equações de Yang-Mills não lineares.

Ainda não comentamos um assunto importantíssimo, na verdade, o que dá sentido a toda a discussão precedente. Precisamos discutir a forma como o campo eletromagnético interage com a matéria, as equações de movimento, i.e.: a força de Lorentz. É claro que uma carga situada em um campo não só é sujeita à ação do campo como também age sobre o campo, transformando-o. Claramente, consideraremos aqui uma carga pequena suficiente para que sua retroação seja insignificante. A fórmula usual para a força de Lorentz $\vec{F}$ sobre uma partícula com carga $q$ com velocidade $v$ é dada por

$$
\vec{F}=q(\vec{E}+\vec{v} \times \vec{B})
$$

\footnotetext{
${ }^{6}$ Lembramos ainda que pela Prop 20 todo fibrado vetorial sobre uma variedade contrátil é isomorfo a uma variedade trivial, ou seja, todas as suas seções podem ser vistas como funções, elementos de $C^{\infty}(U \rightarrow V)$ onde $V$ é a fibra típica.
} 
no entanto, cssa forma não ć abrangente o suficiente para descrever o caso rclativístico. Por exemplo, levando o tempo em consideração da equações de Euler-Lagrange deve emergir também a cncrgia da partícula no campo.

Como definimos o campo eletromagnético, não seria a forçẻsultante simplesmente a ação de $F$ sobre uma dada partícula, i.e.: se v é a velocidade da partícula de carga $q$, na variedade que estamos considerando, deveríamos ter $q(F(\mathbf{v}))^{b}$ para a força exercida na partícula.

Isto de fato se dá, ${ }^{7}$ é possível mostrar de uma forma extraordinariamente simples [14], que no caso relativístico, cm coordenadas, denotando a quadri-velocidade da partícula de carga $q$, por $\mathbf{v}^{\sigma}$, a equação de movimento (em unidades tal que $c=1$ ) toma a forma:

$$
m \frac{d v^{\sigma}}{d s}=F^{\sigma \beta \beta} q v_{\beta}=q F_{\beta}^{\sigma \sigma} v^{\beta}=q(F(\mathbf{v}))^{b}
$$

onde chamamos a atenção que $s$ representa o parâmetro afim da curva da partícula.

Agora escrevendo os (co-)campos em coordenadas temos

$$
\begin{aligned}
& B=B_{x} d y \wedge d z+B_{y} d z \wedge d x+B_{z} d x \wedge d y \\
& E=E_{x} d x+E_{y} d y+E_{z} d z
\end{aligned}
$$

Agora, por (4.6), temos:

$$
F(\mathbf{v})=\left(B_{x} d y \wedge d z+B_{y} d z \wedge d x+B_{z} d x \wedge d y+E_{x} d x \wedge d t+E_{y} d y \wedge d t+E_{z} d z \wedge d t\right)(\mathbf{v})
$$

Escrevendo $\mathbf{v}$ coordenadas, não é difícil calcular que a componente espacial de $q(F(\mathbf{v}))^{b}$ nos dá exatamente a equação de Lorentz, e que sua parte temporal nos fornece $q E_{i} v^{i}=q E(\vec{v})$ que é produto da velocidade pela força que o campo elétrico exerce na partícula, ou seja, a potência inscrida na partícula pelo campo. Terminamos essa seção com uma definição que será ampliada para Kaluza-Klein na próxima seção.

Definition 22 A co-força de Lorentz sobre uma partícula com quadri-velocidade $\mathbf{v}$ é dada por $F(\mathbf{v})$.

\subsection{Kaluza-Klein}

De agora em diante, espaço por si só e tempo por si só, descenderão a meras sombras, e somente uma união dos dois deve sobreviver. - Hermann Minkowski.

\section{História}

Essa frase, dita por Minkowski em seu discurso na 80th Assembléia de Ciência Natural Alemã, é uma das mais famosas citações da física. Ela representa a mudança de paradigma advinda da relatividade: o tempo deixou de ter seu caráter absoluto, mesclou-se às outras

\footnotetext{
${ }^{7}$ Tomando coordenadas $\left\{x_{\mu}\right\}_{\mu=0}^{3}$, onde como sempre: índices romanos significam somente os campos espaciais.
} 
dimensões espaciais, e juntas perderam seu caráter absoluto, rigído, para fornecer uma bela explicação da gravitação ${ }^{8}$.

Em 1919, a teoria eletromagnética de Maxwell estava bem estabelecida, Einstein havia há pouco formulado sua teoria generalizada, enquanto as forças nucleares ainda não eram compreendidas. Era natural portanto, procurar unificar as forças conhecidas pelo aparato teórico da relatividade geral, ou seja através da geometria do espaço-tempo. Theodor Kaluza alcançou essa unificação através de um surpreendente artefato: postular uma dimensão extra. Apesar de atraente, a idéia de Kaluza tinha dois graves defeitos: a dependência da quinta coordenada era suprimida por nenhum motivo aparente, e uma quinta dimensão jamais havia sido observada. Essas duas críticas foram sanadas por Oscar Klein, que postulou uma topologi circular para a quinta dimensão. Ele mostrou que se o raio fosse pequeno o suficiente era possível manter a dependência na quinta coordenada, justificar sua não observabilidade e preservar os resultados de Kaluza. A interpretação deste formalismo seria um pouco distinta da interpretação das teorias de gauge com espaço interno, as partículas realmente percorreriam todas essas dimensões, sendo que o eletromagnetismo emergiria da projeção em nosso espaço-tempo quadri-dimensional dessa dinâmica em 5 dimensões.

Muito anos mais tarde, generalizações de Kaluza-Klein para grupos de dimensẽos maiores deram luz à supergravidade, e ainda depois, como argumenta M.J. Duff [19], a revitalização da teoria de supercordas deveu mais a supergravidade de Kaluza-Klein do que ao modelo de ressonância dual, ao contrário do que revisões históricas normalmente comentam. Faremos um tratamento matemático de um caso mais geral do que Kaluza-Klein original, considerando um fibrado principal com grupo estrutural arbitrário .

\section{Fundamentos Matemáticos}

Ao longo desta seção, $P$ será um $G$-fibrado principal sobre $M$, variedade (pseudo)riemanniana $m$-dimensional. $\left\{X_{i}\right\}_{i=1}^{m}$ será um referencial ortonormal sobre um aberto $\theta \subset M$ e $\left\{\lambda^{i}\right\}_{i=1}^{m}$ seu co-referencial. Novamente $G$ denota um grupo de Lie compacto $k$ dimensional, $\mathfrak{g}$ sua álgebra de Lie dotada de produto interno Ad-invariante $(K)$, como mencionado na Sec $\mathbf{2 . 3}$ e explicitado na Sec. 3.1. O dotaremos de base $\left\{e_{\sigma}\right\}_{\sigma=m+1}^{n}$ (onde $m+k=n$ ) e base dual para $\mathfrak{g}^{*},\left\{e^{\sigma}\right\}_{\sigma=m+1}^{n}$. De uma forma geral os índices latinos variam de 1 a $m$, e os gregos de $m+1$ a $n$. Denotaremos ainda $C_{\sigma \beta}^{\gamma}$ as constantes estruturais de $\mathfrak{g}$ :

$$
\left[e_{\sigma}, e_{\beta}\right]=C_{\sigma \beta}^{\gamma} e_{\gamma}
$$

É claro que $C_{\sigma_{\beta}}^{\gamma}=-C_{\beta_{\sigma}}^{\gamma}$. Ademais, lembrando que como Ad : $G \rightarrow \mathbf{S O}(\mathfrak{g})$ implica $a d:$ $\mathfrak{g} \rightarrow \mathfrak{s o}(\mathfrak{g})$, que são as matrizes anti-simétricas, escrevendo ad $\left(e_{\sigma}\right) e_{\beta}=\left[e_{\sigma}, e_{\beta}\right]$ nós obtemos também que $C_{\sigma \beta}^{\gamma}=-C_{\sigma \gamma}^{\beta}$. Dada uma métrica $h$ em $M$, e uma conexão $H$ (associada a forma $\omega$ ) em $P$, lembramos que, por (2.48) podemos induzir uma única métrica $\alpha$ em $P$ $G$-invariante, tal que a decomposição $T P=\mathrm{H} \oplus \mathrm{V}$ seja ortogonal, que $\left.d \pi\right|_{\mathrm{H}}$ seja isometria, e que o produto interno de vetores verticais seja $K$ :

$$
\alpha=\pi^{*} h+K \circ \omega
$$

\footnotetext{
${ }^{8}$ Segundo o legendário Lev Landau [14] " [a teoria da relatividade geral] representa provavelmente a mais bela de todas as teorias físicas existentes."
} 
Para facilitar a notação, denotarcmos como $c^{i}$ a 1-forma sobre TP dada por $\pi^{*} \lambda^{i}$ (note que os índices são latinos, não gregos) i.e.: $\pi^{*} \lambda^{i} \rightarrow e^{i}$, e identificaremos também $e^{\beta} \circ \omega \rightarrow e^{\beta}$, obtendo uma 1-forma em V. Portanto, deixando as letras latinas maíusculos correrem sobre todos os índices, temos que $\left\{e^{A}\right\}_{A=1}^{n}$ é um co-referencial em $P$. Denotamos o referencial dual a $\left\{e^{A}\right\}_{A=1}^{n}$ pela mótrica $\gamma$ de $P$ por $\left\{c_{A}\right\}_{A=1}^{n}$. É fácil ver que $d \pi\left(e_{i}\right)=X_{i}$. Nós temos tambóm que $\left\{e^{A}\right\}_{A=1}^{n}$ é de fato um co-referencial, já que $e_{\sigma} \in \mathrm{V}$ por definição, e portanto

$$
e^{i}\left(e_{\sigma}\right)=\lambda^{i}\left(d \pi\left(e_{\sigma}\right)\right)=0=\gamma\left(e_{i}, e_{\sigma}\right)=e^{\sigma}\left(e_{i}\right)
$$

Portanto obtemos um pouco mais, que é um referencial ortonormal, e como H é o subfibrado ortogonal a $\mathrm{V}$, nós temos que

$$
\operatorname{span}\left[\left\{c_{\sigma}\right\}_{\sigma=m+1}^{n}\right]=\left.\mathrm{V}\right|_{\theta} \quad \text { e } \quad \operatorname{span}\left[\left\{c_{i}\right\}_{i=1}^{m}\right]=\left.\mathrm{H}\right|_{\theta}
$$

Ternninada essa parte preliminar, o nosso objetivo será calcular a conexão de Levi-Civita em $P$ : a única compatível com a nossa métrica e sem torsão. A partir daí, descobriremos fatos surpreendentes ligados à $P$ e sua relação com $M$. Devemos, em alguns momentos, tentar pensar em $P$ mais como uma variedade riemanniana do que como um fibrado principal.

Seja $\Omega \in \Gamma\left(\Lambda^{2}\left(T P^{*}\right) \otimes \mathfrak{g}\right)$ a forma de curvatura em $P$, que pelo Teo.23 é dada por

$$
\Omega=d \omega \circ \widehat{H}
$$

onde a forma de conexão $\omega$ é nada mais que a projeção no subespaço vertical, seguida do isomorfismo entre este e $\mathfrak{g}$. Como temos um referencial local em V, podemos definir as 2-formas reais $\Omega^{\sigma} \in \Gamma\left(\Lambda^{2}\left(\mathrm{H}^{*}\right)\right)$ :

$$
\Omega^{\sigma} \otimes e_{\sigma}:=\Omega
$$

onde $\Omega^{\sigma}=e^{\sigma}\left(\left[e^{i} \otimes e_{i}, e^{j} \otimes e_{j}\right]\right)$. Ou podemos tomar a descrição ainda mais desmembrada, definindo funçốes reais $F_{i j}^{\sigma}$ :

$$
\left(\frac{1}{2} F_{i j}^{\sigma} e^{j} \wedge e^{i}\right) \otimes e_{\sigma}:=\left(\Omega^{\sigma}\right) \otimes e_{\sigma}
$$

Estamos ficando sem letras para denotar todos os objetos que queremos, então vamos denotar a forma de conexão riemanniana, de Levi-Civita ${ }^{9}$ para $P$, relativa ao referencial que construímos $e_{A}$, como $\widetilde{\omega}_{B}^{A}$. Ou seja

$$
\nabla e_{B}=\widetilde{\omega}_{B}^{A} e_{A} \quad \text { ou equivalentemente } \quad d\left(e^{A}\right)=\widetilde{\omega}_{B}^{A} \wedge e^{B}
$$

Sendo de Levi-Civita, temos que $\widetilde{\omega}_{B}^{A}=-\widetilde{\omega}_{A}^{B}$. Como $\omega=e^{\sigma} \otimes e_{\sigma}$. nós temos que ${ }^{10}$

$$
d \omega=d\left(e^{\sigma}\right) \otimes e_{\sigma}=\widetilde{\omega}_{B}^{\sigma} \wedge e^{B} \otimes e_{\sigma}
$$

E portanto, por (4.19):

$$
\Omega=\widetilde{\omega}_{i}^{\sigma} \wedge e^{i} \otimes e_{\sigma}
$$

\footnotetext{
${ }^{9}$ Que não deve ser confundida com a forma de conexão 's de $P$ como fibrado principal, por exemplo, aqui $\tilde{\tilde{u}^{\prime}} \in \Gamma\left(\Lambda^{1}\left(T P^{*}\right) \otimes \operatorname{End}(T P)\right)$ e portanto não tem valor na álgebra de Lie.

${ }^{10}$ Lembremo-nos que aqui $e_{\sigma}$ éum elemento fixo da álgebra de Lie (ver Teo.23).
} 
O que é um fato curioso, sendo que a priori a conexão de Levi-Civita não precisaria estar relacionada a curvatura da conexão de $P$. A relação existe porque a curvatura da conexão, (sendo a derivada covariante exterior da própria conexão) mede o quanto estes campos variam, o que é claramente relacionado à conexão Levi-Civita.

Comparando (4.22) com (4.20) obtemos

$$
\widetilde{\omega}_{i}^{\sigma} \wedge e^{i}=\frac{1}{2} F_{i j}^{\sigma} e^{j} \wedge e^{i}
$$

Denotando por $\Gamma_{j}^{i}$ a forma de conexão em $M$ relativas ao referencial $X_{i}=d \pi\left(e_{i}\right)$. Chamaremos o pull-back de $\Gamma_{j}^{i}$ de $\bar{\Gamma}_{j}^{i}:=\pi^{*} \Gamma_{j}^{i}$. Nós temos que

$$
d \lambda^{i}=\Gamma_{j}^{i} \wedge \lambda^{j}
$$

Aplicando $\pi^{*}$ em ambos os lados temos:

$$
\begin{aligned}
d\left(e^{i}\right) & =d\left(\pi^{*} \lambda^{i}\right)=\pi^{*} d\left(\lambda^{i}\right)=\pi^{*}\left(\Gamma_{j}^{i} \wedge \lambda^{j}\right) \\
& =\bar{\Gamma}_{j}^{i} \wedge e^{j} \\
& =\widetilde{\omega}_{B}^{i} \wedge e^{B}=\widetilde{\omega}_{j}^{i} \wedge e^{j}+\widetilde{\omega}_{\sigma}^{i} \wedge e^{\sigma}
\end{aligned}
$$

Nós temos ainda, escrevendo explicitamente $d \omega=\Omega-\omega \wedge \omega$ :

$$
\begin{array}{ll}
\left(\widetilde{\omega}_{B}^{\sigma} \wedge e^{B}\right) \otimes e_{\sigma} & =\left(\frac{1}{2} F_{i j}^{\sigma} e^{j} \wedge e^{i}\right) \otimes e_{\sigma}-\frac{1}{2} e^{\beta} \wedge e^{\nu}\left[e_{\beta}, e_{\nu}\right] \\
\therefore \widetilde{\omega}_{B}^{\sigma} \wedge e^{B} & =\frac{1}{2} F_{i j}^{\sigma} e^{j} \wedge e^{i}-\frac{1}{2} C_{\beta \nu}^{\sigma} e^{\beta} \wedge e^{\nu} \\
& =\widetilde{\omega}_{\beta}^{\sigma} \wedge e^{\beta}+\widetilde{\omega}_{i}^{\sigma} \wedge e^{i} \quad \text { mas por (4.23): } \\
\widetilde{\omega}_{i}^{\sigma} \wedge e^{i}=\frac{1}{2} F_{i j}^{\sigma} e^{j} \wedge e^{i} & \Rightarrow \widetilde{\omega}_{\beta}^{\sigma} \wedge e^{\beta}=-\frac{1}{2} C_{\beta \nu}^{\sigma} e^{\beta} \wedge e^{\nu}
\end{array}
$$

Agora, as contas já estão maduras o suficiente para introduzirmos os análogos dos símbolos de Christoffel, definindo:

$$
\widetilde{\omega}_{B C}^{A}:=\widetilde{\omega}_{B}^{A}\left(e_{C}\right) \Longrightarrow \widetilde{\omega}_{B}^{A}=\widetilde{\omega}_{B C}^{A} e^{C}
$$

Introduzindo nas duas últimas equações de (4.26), como temos uma base completa de 2formas, obtemos sem maiores esforços que

$$
\widetilde{\omega}_{i j}^{\sigma} e^{j} \wedge e^{i}=\frac{1}{2} F_{i j}^{\sigma} e^{j} \wedge e^{i}
$$

portanto $\widetilde{\omega}_{i \beta}^{\sigma}=0=\widetilde{\omega}_{\sigma \beta}^{i}$ e finalmente

$$
\widetilde{\omega}_{i}^{\sigma}=\frac{1}{2} F_{i j}^{\sigma} e^{j}
$$

Da mesma forma obtemos que $\widetilde{\omega}_{\beta i}^{\sigma}=0 \mathrm{e}$

$$
\widetilde{\omega}_{\beta}^{\sigma}=-\frac{1}{2} C_{\nu \beta}^{\sigma} e^{\nu}
$$


Substituindo (4.27) em (4.25) obtemos:

$$
\widetilde{\omega}_{j}^{i} \wedge e^{j}=\bar{\Gamma}_{j}^{i} \wedge e^{j}+\frac{1}{2} \sum_{\sigma} F_{i j}^{\sigma} e^{j} \wedge e^{\sigma}=\bar{\Gamma}_{j}^{i} \wedge e^{j}-\frac{1}{2} \sum_{\sigma} F_{i j}^{\sigma} e^{\sigma} \wedge e^{j}
$$

e estamos prontos para enunciar o primeiro resultado obtido:

Proposition 27

$$
\left\{\begin{array}{l}
\widetilde{\omega}_{j}^{i}=\bar{\Gamma}_{j}^{i}-\frac{1}{2} \sum_{\sigma} F_{i j}^{\sigma} e^{\sigma} \\
\widetilde{\omega}_{i}^{\sigma}=\frac{1}{2} F_{i j}^{\sigma} e^{j} \\
\widetilde{\omega}_{\beta}^{\sigma}=-\frac{1}{2} C_{\nu \beta}^{\sigma} e^{\nu}
\end{array}\right.
$$

Agora, suponhamos que $\gamma: I \rightarrow P$ seja uma geodésica e $\bar{\gamma}$ sua projeção em $M$. Definimos uma função $q: I \rightarrow \mathfrak{g}$ chamada de carga específica por $q(t)=\omega\left(\gamma^{\prime}(t)\right)$. Note que esse nome garboso nada mais significa que a projeção da velocidade em V (ou $\mathfrak{g}$ ), e tentaremos provar que a projeção da velocidade da partícula nas dimensões extras é sua carga, e que portanto deve ser mantida constante para geodésicas. Lembremos também que no modelo considerado, as partículas sempre percorrem geodésicas, pois não estamos considerando forças não inclusas em nossa geometria (i.e.: forças externas).

Já que $\omega=e_{\sigma} \otimes e^{\sigma}$ então para qualquer vetor $v$ em $P$ nós temos que

$$
\omega(v)=\left\langle v, e_{\sigma}\right\rangle e^{\sigma}
$$

Para provar que para uma geodésica $\gamma(t)$ em $P$ a carga específica $q(t)=\omega\left(\gamma^{\prime}(t)\right)$ é constante, basta mostrar então que

$$
\left\langle\gamma^{\prime}(t), e_{\sigma}\right\rangle=\text { cte }
$$

Mas a métrica que escolhemos para $P$ é invariante pela ação de $G$, então como $e^{\sigma}$ são os campos tangentes à órbita do grupo, eles são campos de Killing. Logo a constância de $\left\langle\gamma^{\prime}(t), e_{\sigma}\right\rangle$ é um caso especial do seguinte teorema:

Theorem 29 Se $X$ é um campo de Killing em uma variedade riemanniana $N$ e $\gamma$ é uma geodésica em $N$ então o produto interno de $X$ com $\gamma^{\prime}(\iota)$ independe de $l$.

Dem: Necessitaremos da primeira fórmula da variação:

Seja $\gamma: I \rightarrow N$ uma curva suave e

$$
\begin{aligned}
& F:[-\epsilon, \epsilon] \times I \rightarrow N \\
& (s, t) \longmapsto F(s: t)
\end{aligned}
$$

uma variação de $\gamma$, i.e.: tal que $F(0, t)=\gamma(t)$ exatamente como construímos para a prova do Teo. 14. tal que, para $s$ fixo: $F(s, t)=F_{s}(t)=\gamma_{s}(t)$ Agora sejam

$$
{\frac{\partial}{\partial s \mid\left(s_{0}, t_{0}\right)}}_{:=F_{*}} \frac{d}{d s \mid\left(s_{0}, t_{0}\right)} \quad ; \quad{\frac{\partial}{\partial t \mid\left(s_{0}, t_{0}\right)}}:=F_{*} \frac{d}{d t \mid\left(s_{0}, t_{0}\right)}
$$


Como não temos torsão, e $s, t$ são coordenadas,

$$
\frac{D}{d s} \frac{\partial}{\partial t}=\frac{D}{d t} \frac{\partial}{\partial s}
$$

Para um dado $s$ temos:

$$
E(F(s, t))=\int_{a}^{b}\left\langle\frac{\partial}{\partial t} \mid(s, t), \frac{\partial}{\partial t}{ }_{\mid(s, t)}\right\rangle d t
$$

Então, temos

$$
\begin{aligned}
\frac{1}{2} \frac{d}{d s} E(F(s, t)) & =\frac{1}{2} \frac{d}{d s} \int_{a}^{b}\left\langle\frac{\partial}{\partial t_{\mid(s, t)}}, \frac{\partial}{\partial t_{\mid(s, t)}}\right\rangle d t=\int_{a}^{b}\left\langle\frac{D}{d s} \frac{\partial}{\partial t_{\mid(s, t)}}, \frac{\partial}{\partial t_{\mid(s, t)}}\right\rangle d t \\
& =\int_{a}^{b}\left\langle\frac{D}{d t} \frac{\partial}{\partial s_{\mid(s, t)}}, \frac{\partial}{\partial t_{\mid(s, t)}}\right\rangle d t=\left.\left\langle\frac{\partial}{\partial s_{\mid(s, t)}}, \frac{\partial}{\partial t_{\mid(s, t)}}\right\rangle\right|_{a} ^{b}-\int_{a}^{b}\left\langle\frac{\partial}{\partial s \mid(s, t)}, \frac{D}{d t} \frac{\partial}{\partial t_{\mid(s, t)}}\right\rangle d t
\end{aligned}
$$

Em particular,

$$
\left.\frac{1}{2} \frac{d}{d s} E(F(s, t))\right|_{s=0}=\left.\left\langle\frac{\partial}{\partial s}{ }_{\mid(0, t)}, \gamma^{\prime}(t)\right\rangle\right|_{a} ^{b}-\int_{a}^{b}\left\langle\frac{\partial}{\partial s}{ }_{\mid(0, t)}, \frac{D}{d t} \gamma^{\prime}(t)\right\rangle d t
$$

Se $\gamma$ é geodésica então:

$$
\left.\frac{1}{2} \frac{d}{d s} E(F(s, t))\right|_{s=0}=\left.\left\langle\frac{\partial}{\partial s \mid(0, t)}, \gamma^{\prime}(t)\right\rangle\right|_{a} ^{b}
$$

Agora suponhamos que $\Phi_{s}$ seja o subgrupo a 1-parâmetro de isometrias gerado por $X$, fazemos então

$$
F(s, t)=\Phi_{s}(\gamma(t))
$$

Agora

e como $\Phi_{s}$ é isometria:

$$
F_{*} \frac{d}{d t \mid(s, t)}=\left(\Phi_{s}\right)_{*}\left(\gamma^{\prime}(t)\right)=\frac{\partial}{\partial t}_{\mid(s, t)}
$$

$$
\left\langle\left(\Phi_{s}\right)_{*}\left(\gamma^{\prime}(t)\right),\left(\Phi_{s}\right)_{*}\left(\gamma^{\prime}(t)\right)\right\rangle \text { não depende de } s
$$

portanto

e finalmente

$$
\left.\frac{1}{2} \frac{d}{d s} E(F(s, t))\right|_{s=0}=0
$$

$$
\left.\left\langle\frac{\partial}{\partial s_{\mid(0, t)}}, \gamma^{\prime}(t)\right\rangle\right|_{b}=\left.\left\langle\frac{\partial}{\partial s_{\mid(0, t)}}, \gamma^{\prime}(t)\right\rangle\right|_{a}
$$

para todo $[a, b] \subset I$. Ou seja, o produto interno de $X$ com $\gamma^{\prime}(t)$ independe de $t$.

Uma forma mais direta de obtermos este resultado seria assumindo que um campo de Killing $X$ obedece, para quaisquer campos $Y$ e $Z$ :

$$
\left\langle\nabla_{Y} X, Z\right\rangle+\left\langle\nabla_{Z} X, Y\right\rangle=0
$$


e portanto como $\gamma$ ć gcodésica

$$
\frac{d}{d t}\left\langle X, \gamma^{\prime}(t)\right\rangle=\left\langle\nabla_{\gamma^{\prime}(t)} X, \gamma^{\prime}(t)\right\rangle=0
$$

nós fizemos a primeira prova porque a consideramos mais geométrica, menos algébrica (além do quê, simultâneamente mostra que $X_{\gamma}$ é um campo de Jacobi, i.e.: $e^{\sigma} \mid \gamma$ são campos de Jacobi, o que pode vir a ser útil).

Agora definimos um funcional linear sobre a projeção da nossa curva $\bar{\gamma}(t)=\pi(\gamma(t)$, ou seja, sobre $T_{\bar{\gamma}(t)} M I$, da seguinte forma

Definition $23 A$ co-força de Lorentz, $\eta(\gamma(t))$ é definida por:

$$
\begin{aligned}
\eta(\gamma(t)): & T_{\bar{\gamma}(l)} M \rightarrow \mathbb{R} \\
& v \mapsto K\left(\Omega_{\gamma(t)}\left(\widetilde{v}, \hat{H}\left(\gamma^{\prime}(t)\right)\right), \omega\left(\gamma^{\prime}(t)\right)\right)
\end{aligned}
$$

onde $K$ é o produto interno em $\mathfrak{g}$, $\widetilde{v}$ é o levantamento horizontal de $v$ e $\widehat{H}$ é a projeção no subfibrado horizontal.

Notemos que

$$
\Omega_{\gamma(t)}\left(\widetilde{v}, \widehat{H}\left(\gamma^{\prime}(t)\right)\right)=\Omega_{\gamma(t)}\left(\widetilde{v}, \gamma^{\prime}(t)\right)
$$

Totemos ainda que esta expressão fica um pouco mais complicada devido ao uso do levantamento horizontal do vetor $v$, que é necessário já que queremos ver os efeitos em $M$ e não em $P$. Se definíssemos $v$ como projeção de um vetor qualquer $X$, teríamos simplesmente $\Omega_{\gamma(t)}\left(X: \gamma^{\prime}(t)\right)$.

Agora lembramos que provamos que a carga específica é uma constante, e portanto a forma de Killing $K$ aqui é só uma maneira de multiplicar pela carga. Finalmente, associando a curvatura da conexão com o campo, assim como fizemos na seção anterior, temos que a força de Lorentz aqui é a projeção da força eletromagnética na partícula sobre a direção $X$, ou melhor sobre a direção $d \pi(X)$. Se retirarmos a direção $X$ (ou $v$ ), nós temos que (substituindo a notação $K$ por ".")

$$
\eta\left(\gamma^{\prime}(t)\right)=\Omega\left(\gamma^{\prime}(t)\right) \cdot q
$$

é uma 1-forma, equivalente a co-força de Lorentz, que nos fornece também a direção com que o campo eletromagnético atua sobre a partícula. Escrevendo em termos de nossa base $\left\{e_{\Lambda}\right\}$, temos,

$$
\gamma^{\prime}(t)=u^{i} e_{i}+q^{\sigma} e_{\sigma}
$$

onde claramente ${ }^{11}$

$$
q^{\sigma} e_{\sigma}=\omega\left(\gamma^{\prime}(\iota)\right)=q \quad \text { e } \quad \bar{\gamma}(l)=u^{i} X_{i}
$$

Agora, por (4.20), $\Omega=\frac{1}{2} F_{i j}^{\sigma} c^{i} \wedge e^{i} \otimes e_{\sigma}$, portanto, como $\left\{e_{\sigma}\right\}$ ć basc ortonormal (cm rclação a $K$ ) para $\mathfrak{g}$, temos:

\footnotetext{
${ }^{11}$ Agora que nossa compreensão física do assunto já se aprofundou minimamente: vale a pena apontar que $\gamma(t)$ tem $n-m$ "cargas", distintas, que não se misturam e não se alteram. Humm....
} 


$$
\begin{aligned}
\Omega\left(\gamma^{\prime}(t)\right) \cdot q & =\frac{1}{2} \sum_{\sigma} F_{i j}^{\sigma} u^{i} q^{\sigma} e^{j} \\
\therefore \quad\left(\Omega\left(\gamma^{\prime}(t)\right) \cdot q\right)^{b} & =\frac{1}{2} \sum_{\sigma, j} F_{i j}^{\sigma} u^{i} q^{\sigma} e_{j}
\end{aligned}
$$

E cuja projeção corresponde a

$$
F\left(\bar{\gamma}^{\prime}(t)\right) \cdot q=\frac{1}{2} \sum_{\sigma, j} F_{i j}^{\sigma} u^{i} q^{\sigma} X_{j}
$$

De fato, qualquer que seja $v \in T_{\bar{\gamma}(t)} M, v=v^{i} X_{i}$, e portanto $\widetilde{v}=v^{i} e_{i}$ e nós temos que tanto faz aplicarmos a força de Lorentz como definida em $M$, o que chamamos de $F$, ou no levantamento horizontal, em $P$ (além disso é claro que, como as bases são duais pela métrica, as ccomponentes da descrição como formas ou como campos são as mesmas).

É claro que ainda não provamos o principal, i.e.:

Theorem 30 Há uma discrepância entre a equação da geodésica em $M$ e a projeção da geodésica em $P$ que é exatamente a força de Lorentz:

Dem: A demonstração consiste em calcular a projeção de

$$
\frac{D \gamma^{\prime}(t)}{d t}=\nabla_{\gamma^{\prime}(t)}^{P} \gamma^{\prime}(t) \text { e compará-la a } \frac{D \bar{\gamma}^{\prime}(t)}{d t}=\nabla_{\bar{\gamma}^{\prime}(t)}^{M} \bar{\gamma}^{\prime}(t)
$$

Escrevendo $\gamma^{\prime}(t)=u^{i} e_{i}+q^{\sigma} e_{\sigma}$, temos calculando e em seguida projetando no referencial espacial $^{12}$ :

$$
\begin{aligned}
\nabla_{\gamma^{\prime}(t)}^{P} \gamma^{\prime}(t) & =u^{j} \nabla_{e_{j}}^{P}\left(u^{i} e_{i}+q^{\sigma} e_{\sigma}\right)+q^{\beta} \nabla_{e_{\beta}}^{P}\left(u^{i} e_{i}+q^{\sigma} e_{\sigma}\right) \\
& =u^{j}\left(d u^{i}\left(e_{j}\right) e_{i}+u^{i} \widetilde{\omega}_{i}^{A}\left(e_{j}\right) e_{A}\right)+u^{j} q^{\sigma} \widetilde{\omega}_{\sigma}^{A}\left(e_{j}\right) e_{A}+q^{\beta} u^{i} \widetilde{\omega}_{i}^{A}\left(e_{\beta}\right) e_{A}+q^{\beta} q^{\sigma} \widetilde{\omega}_{\sigma}^{A}\left(e_{\beta}\right) e_{A} \\
e^{i}\left(\nabla_{\gamma^{\prime}(t)}^{P} \gamma^{\prime}(t)\right) & =u^{j} d u^{i}\left(e_{j}\right)+u^{j} u^{k} \widetilde{\omega}_{k}^{i}\left(e_{j}\right)+u^{j} q^{\sigma} \widetilde{\omega}_{\sigma}^{i}\left(e_{j}\right)+u^{j} q^{\beta} \widetilde{\omega}_{j}^{i}\left(e_{\beta}\right)+q^{\beta} q^{\sigma} \widetilde{\omega}_{\sigma}^{i}\left(e_{\beta}\right)
\end{aligned}
$$

Agora utilizando (4.30), e a anti-simetria de $\widetilde{\omega}_{A}^{B}$ temos que

$$
\begin{aligned}
u^{j} u^{k} \widetilde{\omega}_{k}^{i}\left(e_{j}\right) & =u^{j} u^{k} \bar{\Gamma}_{k}^{i}\left(e_{j}\right) \\
u^{j} q^{\sigma} \widetilde{\omega}_{\sigma}^{i}\left(e_{j}\right) & =-\frac{1}{2} \sum_{\sigma} u^{j} q^{\sigma} F_{i j}^{\sigma} \\
u^{j} q^{\beta} \widetilde{\omega}_{j}^{i}\left(e_{\beta}\right) & =-\frac{1}{2} \sum_{\beta} u^{j} q^{\beta} F_{i j}^{\beta} \\
q^{\beta} q^{\sigma} \widetilde{\omega}_{\sigma}^{i}\left(e_{\beta}\right) & =0
\end{aligned}
$$

\footnotetext{
${ }^{12}$ Note que não são necessariamente campos coordenados, então deve-se resistir ao hábito de usar este termo ao longo dessa seção. Utilizaremos fortemente a notação de soma de Einstein, muitas vezes mudando índices repetidos (i.e.: que são mudos) de nome.
} 
E finalmont.c, substituindo

$$
\begin{aligned}
e^{i}\left(\nabla_{\gamma^{\prime}(t)}^{P} \gamma^{\prime}(t)\right) & =\left(u^{j} d u^{i}\left(e_{j}\right)+u^{j} u^{k} \bar{\Gamma}_{k}^{i}\left(e_{j}\right)\right)-\sum_{\sigma} u^{j} q^{\sigma} F_{i j}^{\sigma} \\
=\pi^{*} \lambda^{i}\left(\nabla_{\gamma^{\prime}(t)}^{P} \gamma^{\prime}(t)\right) & =\lambda^{i}\left(u^{j} d u^{i}\left(X_{j}\right)+u^{j} u^{k} \Gamma_{k}^{i}\left(X_{j}\right)\right)-\sum_{\sigma} u^{j} q^{\sigma} F_{i j}^{\sigma} \\
& =\lambda^{i}\left(u^{j} \nabla_{X_{j}}^{M}\left(u^{k} X_{k}\right)\right)-\sum_{\sigma} u^{j} q^{\sigma} F_{i j}^{\sigma} \\
& =\lambda^{i}\left(\nabla_{\bar{\gamma}^{\prime}(t)}^{M} \bar{\gamma}^{\prime}(t)\right)-\sum_{\sigma} u^{j} q^{\sigma} F_{i j}^{\sigma}
\end{aligned}
$$

Este resultado significa que habitantes de $M$ que não soubessem da existência de outras dimensões, não saberiam que na verdade as geodésicas que percorrem estão em um espaço com maior dimensionalidade. Encontrariam certas características das partículas que perceberiam permanecer constantes, e as chamariam de carga, cor, charme, ou qualquer outro nome estranho que escolhessem. Perceberiam também que a discrepância entre as trajetórias que as partículas deveriam percorrer segundo suas inércias (geodésicas), era proporcional a essas cargas de uma maneira específica, de acordo com uma lei que não envolvia explicitamente outras dimensões.

No entanto, o objetivo da Teoria de kaluza-Klein, é a de derivar as equações de Einstein e de Yang-Mills a partir das equações de Einstein em uma dimensionalidade maior. Logo nós precisamos derivar estas equações de campo a partir da extremalização da integral do escalar de curvatura ${ }^{P} R$.

Agora, $P$ tem uma curvatura escalar ${ }^{P} R$ que pela invariância da métrica é constante $\mathrm{cm}$ órbitias de $G$, e portanto pode scr calculada como função rcal sobre $M$. Alóm disso, a existência de uma métrica Ad-invariante em $\mathfrak{g}$ é equivalente à existência de uma métrica bi-invariante em $G$ (por translação), logo $G$ tem uma curvatura escalar que é claramente, constante [20]; e também bem definida sobre cada ponto de $M$. Finalmente, como tanto $M$ quanto $\mathfrak{g}$ tem métrica, e $\Omega$ é uma 2 -forma em $M$ a valores em $\mathfrak{g}, \Omega$ tem uma norma bem definida

$$
\|\Omega\|^{2}=\sum_{\sigma, i, j}\left(F_{i j}^{\sigma}\right)^{2}
$$

que também é função escalar em $M$. Acharemos uma relação entre a curvatura do espaço total, ${ }^{P} R$, a do espaço base, ${ }^{M} R$, a do grupo, ${ }^{G} R$, e a do cammo eletromagnético $\|\Omega\|$.

Theorem 31 A relação entre as curvaturas escalures é dada por ${ }^{13}$ :

$$
{ }^{P} R={ }^{M} R-\frac{1}{2}\|\Omega\|^{2}+{ }^{G} R
$$

\footnotetext{
${ }^{13}$ Notemos que mudamos livremente índices para cima e para baixo, ou seja, não mantemos a lei de conservação dos índices. Isso ocorre porque estamos em um referencial ortonormal, cujo dual é feito também pela métrica, ou seja $\left\langle\cdot, e_{A}\right\rangle=e^{A}$, onde obviamente violamos a conservação.
} 
Dem: Nós sabemos que

$$
{ }^{P} \Omega\left(e_{A}, e_{B}\right) e_{C}={ }^{P} R_{A B C}^{D} e_{D} \text { ou ainda }{ }^{P} R_{A B C D}=\left\langle{ }^{P} \Omega\left(e_{A}, e_{B}\right) e_{C}, e_{D}\right\rangle
$$

onde ${ }^{P} \Omega=d \widetilde{\omega}+\widetilde{\omega} \wedge \widetilde{\omega} \in \Gamma\left(\Lambda^{2}(T P) \otimes \operatorname{End}(T P)\right)$, e que, a exemplo das conexões $\omega$ e $\widetilde{\omega}$, não deve ser confundida com $\Omega$. Agora vejamos:

$$
\begin{aligned}
{ }^{P} R: & =\sum_{A, B}{ }^{P} R_{A B A B}=\sum_{A, B}\left\langle{ }^{P} \Omega\left(e_{A}, e_{B}\right) e_{A}, e_{B}\right\rangle \\
& =\sum_{A, B}\left\langle d \widetilde{\omega}\left(e_{A}, e_{B}\right) e_{A}, e_{B}\right\rangle+\left\langle\left(\widetilde{\omega} \wedge \widetilde{\omega}\left(e_{A}, e_{B}\right)\right) e_{A}, e_{B}\right\rangle
\end{aligned}
$$

Primeiramente ${ }^{14}$, escrevemos $\widetilde{\omega}=\widetilde{\omega}_{D}^{C} e^{C} \otimes e_{C}$, portanto $d \widetilde{\omega}=\left(d \widetilde{\omega}_{D}^{C}\right) e^{D} \otimes e_{C} \operatorname{assim}^{15}$ temos

$$
\left\langle d \widetilde{\omega}\left(e_{A}, e_{B}\right) e_{A}, e_{B}\right\rangle=\left(d \widetilde{\omega}_{D}^{C}\right)\left(e_{A}, e_{B}\right) e^{D}\left(e_{A}\right)\left\langle e_{C}, e_{B}\right\rangle=\left(d \widetilde{\omega}_{A}^{B}\right)\left(e_{A}, e_{B}\right)
$$

Similarmente $\widetilde{\omega} \wedge \widetilde{\omega}=\widetilde{\omega}_{D}^{C} \wedge \widetilde{\omega}_{F}^{E} e_{C} \otimes e^{D}\left(e_{E}\right) \otimes e^{F}=\widetilde{\omega}_{D}^{C} \wedge \widetilde{\omega}_{F}^{D} e_{C} \otimes e^{F}$, assim como explicitado em (1.30). Claramente

$$
\left\langle\left(\widetilde{\omega} \wedge \widetilde{\omega}\left(e_{A}, e_{B}\right)\right) e_{A}, e_{B}\right\rangle=\widetilde{\omega}_{D}^{B} \wedge \widetilde{\omega}_{A}^{D}\left(e_{A}, e_{B}\right)
$$

Antes de mais nada, afirmamos que a curvatura de $G$, equipado com métrica bi-invariante é $\frac{1}{4} C_{\alpha \beta}^{\sigma} C_{\alpha \beta}^{\sigma}$. Um caminho das pedras para a demonstração, é, assumindo que quando temos uma métrica bi-invariante em $G$ vale ${ }^{G} \nabla_{X} Y=\frac{1}{2}[X, Y]$, encontrar ${ }^{G} R(X, Y) Z=\frac{1}{4}[[X . Y], Z]$ e daí segue que ${ }^{G} R=\frac{1}{4}\left\langle\left[e_{\alpha}, e_{\beta}\right],\left[e_{\alpha}, e_{\beta}\right]\right\rangle$ de onde segue nossa proposição.

Continuando, nós temos, a partir de (4.35) e (4.34):

$$
\begin{aligned}
\widetilde{\omega}_{D}^{B} \wedge \widetilde{\omega}_{A}^{D}\left(e_{A}, e_{B}\right) & =\widetilde{\omega}_{D}^{B}\left(e_{A}\right) \widetilde{\omega}_{A}^{D}\left(e_{B}\right)-\widetilde{\omega}_{D}^{B}\left(e_{B}\right) \widetilde{\omega}_{A}^{D}\left(e_{A}\right) \\
d \widetilde{\omega}_{A}^{B}\left(e_{A}, e_{B}\right) & =e_{B}\left[\widetilde{\omega}_{A}^{B}\left(e_{A}\right)\right]-e_{A}\left[\widetilde{\omega}_{A}^{B}\left(e_{B}\right)\right]-\widetilde{\omega}_{A}^{B}\left(\left[e_{A}, e_{B}\right]\right)
\end{aligned}
$$

Agora, a curvatura de $M$ será dada por uma expressão idêntica a de $P$, substituindo $\widetilde{\omega}$ por $\Gamma$. Para (4.36), pela anti-simetria em $A$ e $B$, precisamos considerar três casos, $A, B$ espaciais, $A, B$ "gregos" e $A$ espacial, $B$ grego. Substiuindo $A, B$ e $D$ em (4.36) por índices espaciais, nós obtemos a equação análoga para $M$ :

$$
\widetilde{\omega}_{k}^{j} \wedge \widetilde{\omega}_{i}^{k}\left(e_{i}, e_{j}\right)=\bar{\Gamma}_{k}^{j} \wedge \bar{\Gamma}_{i}^{k}\left(e_{i}, e_{j}\right)=\Gamma_{k}^{j}\left(X_{i}\right) \Gamma_{i}^{k}\left(X_{j}\right)-\Gamma_{k}^{j}\left(X_{j}\right) \Gamma_{i}^{k}\left(X_{i}\right)
$$

Nos restaram da somatória os termos para $D$ grego, por (4.30), lembrando que $F_{i j}^{\sigma}$ é antisimétrico em $i, j$ :

$$
\begin{aligned}
\widetilde{\omega}_{\sigma}^{j} \wedge \widetilde{\omega}_{i}^{\sigma}\left(e_{i}, e_{j}\right) & =\widetilde{\omega}_{\sigma}^{j}\left(e_{i}\right) \widetilde{\omega}_{i}^{\sigma}\left(e_{j}\right)-\widetilde{\omega}_{\sigma}^{j}\left(e_{j}\right) \widetilde{\omega}_{i}^{\sigma}\left(e_{i}\right) \\
& =\widetilde{\omega}_{j}^{\sigma}\left(e_{i}\right) \widetilde{\omega}_{i}^{\sigma}\left(e_{j}\right) \\
& =-\frac{1}{4} F_{i j}^{\sigma} F_{i j}^{\sigma}
\end{aligned}
$$

\footnotetext{
${ }^{14} \mathrm{~A}$ partir de agora não colocaremos mais explicitamente o sinal da somatória quando índices se repetirem à mesma altura.

${ }^{15}$ Note que a derivada exterior não se aplica em $e^{D}$, isso ocorre porque a derivada exterior nesse caso, tem um significado específico, como mostramos na equação (1.70), onde tínhamos uma forma em $M$ a valores no fibrado vetorial, e a derivada exterior só atuava na forma em $M$. Isso não muda se o fibrado for $T M$ (ou , no nosso caso, $T P$ ).
} 
Faltam dois casos para terminarmos de analisar (4.36). Para $\Lambda, B$ e $D$ gregos nós t.cmos que

$$
\begin{aligned}
\widetilde{\omega}_{\beta}^{\sigma} \wedge \widetilde{\omega}_{\alpha}^{\beta}\left(e_{\alpha}, e_{\sigma}\right) & =\widetilde{\omega}_{\beta}^{\sigma}\left(e_{\alpha}\right) \widetilde{\omega}_{\alpha}^{\beta}\left(e_{\sigma}\right)-\widetilde{\omega}_{\beta}^{\sigma}\left(e_{\sigma}\right) \widetilde{\omega}_{\alpha}^{\beta}\left(e_{\alpha}\right) \\
& =\widetilde{\omega}_{\beta}^{\sigma}\left(e_{\alpha}\right) \widetilde{\omega}_{\alpha}^{\beta}\left(e_{\sigma}\right)=\frac{1}{4} C_{\alpha \beta}^{\sigma} C_{\sigma \alpha}^{\beta} \\
& =-\frac{1}{4} C_{\beta \alpha}^{\sigma} C_{\beta \alpha}^{\sigma}
\end{aligned}
$$

Para $D$ espacial, é fácil verificar que :

$$
\widetilde{\omega}_{i}^{\sigma} \wedge \tilde{\omega}_{\alpha}^{i}\left(e_{\alpha}, e_{\sigma}\right)=0
$$

Para $A$ espacial $B, D$ gregos temos

$$
\begin{aligned}
\widetilde{\omega}_{\sigma}^{\beta} \wedge \widetilde{\omega}_{i}^{\sigma}\left(e_{i}, e_{\beta}\right) & =\widetilde{\omega}_{\sigma}^{\beta}\left(e_{i}\right) \widetilde{\omega}_{i}^{\sigma}\left(e_{\beta}\right)-\widetilde{\omega}_{\sigma}^{\beta}\left(e_{\beta}\right) \widetilde{\omega}_{i}^{\sigma}\left(e_{i}\right) \\
& =-\widetilde{\omega}_{\sigma}^{\beta}\left(e_{\beta}\right) \widetilde{\omega}_{i}^{\sigma}\left(e_{i}\right)=0
\end{aligned}
$$

Pela anti-simetria de $C_{\alpha \beta}^{\sigma}$. Para $A, D$ espaciais e $B$ grego obtemos assim como em (4.39)(4.41):

$$
\widetilde{\omega}_{j}^{\sigma} \wedge \widetilde{\omega}_{i}^{j}\left(e_{i}: e_{\sigma}\right)=\widetilde{\omega}_{j}^{\sigma}\left(e_{i}\right) \tilde{\omega}_{i}^{j}\left(e_{\sigma}\right)-\widetilde{\omega}_{j}^{\sigma}\left(e_{\sigma}\right) \widetilde{\omega}_{i}^{j}\left(e_{i}\right)=-\frac{1}{4} F_{i j}^{\sigma} F_{i j}^{\sigma}
$$

Agora, seguindo para a equação (4.37). Para $A, B$ gregos temos:

$$
\begin{aligned}
d \widetilde{\omega}_{\alpha}^{\beta}\left(e_{\alpha}, e_{\beta}\right) & =e_{\beta}\left[\widetilde{\omega}_{\alpha}^{\beta}\left(e_{\alpha}\right)\right]-e_{\alpha}\left[\widetilde{\omega}_{\alpha}^{\beta}\left(e_{\beta}\right)\right]-\widetilde{\omega}_{\alpha}^{\beta}\left(\left[e_{\alpha}, e_{\beta}\right]\right) \\
& =-\widetilde{\omega}_{\alpha}^{\beta}\left(\left[e_{\alpha}, e_{\beta}\right]\right)=-C_{\beta \alpha}^{\sigma} \widetilde{\omega}_{\alpha}^{\beta}\left(e_{\sigma}\right) \\
& =\frac{1}{2} C_{\beta \alpha}^{\sigma} C_{\beta \alpha}^{\sigma}
\end{aligned}
$$

Onde utilizamos a constância e a anti-simetria de $C_{\alpha \beta}^{\sigma}$ e (4.30). Para $B$ grego e $A$ espacial temos

$$
\begin{aligned}
d \widetilde{\omega}_{i}^{\beta}\left(c_{i}, c_{\beta}\right) & =d\left(\frac{1}{2} F_{i j}^{\beta} c^{j}\right)\left(c_{i}, c_{\beta}\right)=\frac{1}{2}\left(d F_{i j}^{\beta} \wedge c^{j}+F_{i j}^{\beta} d c^{j}\right)\left(c_{i}, c_{\beta}\right) \\
& =-\frac{1}{2} d F_{i i}^{\beta}\left(e_{\beta}\right)+\frac{1}{2} F_{i j}^{\beta}\left(\widetilde{\omega}_{A}^{j} \wedge e^{A}\left(e_{i}, e_{\beta}\right)\right) \\
& =\frac{1}{2} F_{i j}^{\beta}\left(\widetilde{\omega}_{\beta}^{j}\left(e_{i}\right)-\widetilde{\omega}_{i}^{j}\left(e_{\beta}\right)\right) \\
& =-\frac{1}{2} F_{i j}^{\beta} F_{i j}^{\beta}
\end{aligned}
$$

Onde utilizamos anti-simetria para vermos que $d F_{i i}^{\beta}=0$.

Finalmente, para os bravos que conseguiram suportar essa infinitude de contas, chegamos 
ao caso final: $A, B$ espacial.

$$
\begin{aligned}
d \widetilde{\omega}_{j}^{i}\left(e_{j}, e_{i}\right) & =d\left(\bar{\Gamma}_{j}^{i}-\frac{1}{2} F_{i j}^{\sigma} e^{\sigma}\right)\left(e_{j}, e_{i}\right) \\
& =\left(d \bar{\Gamma}_{j}^{i}-\frac{1}{2} d F_{i j}^{\sigma} \wedge e^{\sigma}-\frac{1}{2} F_{i j}^{\sigma} d e^{\sigma}\right)\left(e_{i}, e_{j}\right) \\
& =d \bar{\Gamma}_{j}^{i}\left(e_{i}, e_{j}\right)-\frac{1}{2} F_{i j}^{\sigma}\left(\widetilde{\omega}_{A}^{\sigma}\left(e_{i}\right) e^{A}\left(e_{j}\right)-\widetilde{\omega}_{A}^{\sigma}\left(e_{j}\right) e^{A}\left(e_{i}\right)\right) \\
& =d \bar{\Gamma}_{j}^{i}\left(e_{i}, e_{j}\right)-\frac{1}{2} F_{i j}^{\sigma}\left(\widetilde{\omega}_{j}^{\sigma}\left(e_{i}\right)-\widetilde{\omega}_{i}^{\sigma}\left(e_{j}\right)\right) \\
& =d \bar{\Gamma}_{j}^{i}\left(e_{i}, e_{j}\right)+\frac{1}{2} F_{i j}^{\sigma} F_{i j}^{\sigma}
\end{aligned}
$$

Agora, somando (4.55) a (4.60) obtemos $d \bar{\Gamma}_{j}^{i}\left(e_{i}, e_{j}\right)$ que somado a (4.38) nos fornece justamente ${ }^{M} R$. Agora, somando (4.44) a (4.51) obtemos ${ }^{G} R$, e finalmente, somando (4.41) a (4.48) obtemos $-\frac{1}{2}\|\Omega\|^{2}$.

Agora, como vimos na derivação da equação (2.48), há uma bijeção entre as métricas de fibrado (bundle metrics) para $P$ e $(h, \omega)$ onde $h$ é métrica de $M$ e $\omega$ é forma de conexão em $P$. Logo, ao invés de variarmos o funcional de ação em relação a métrica, podemos tomar a variação independente de $h$ e $\omega$. Então temos :

$$
S(\alpha)=S(h, \omega)=\int_{P}{ }^{P} R \operatorname{vol}_{P}=\int_{M}\left({ }^{M} R-\frac{1}{2}\langle\Omega, \Omega\rangle+{ }^{G} R\right) \operatorname{vol}_{M}
$$

Lembramos que métrica $H$ entra sorrateiramente em $\langle\Omega, \Omega\rangle$ através do operador $*$ de Hodge. É possível mostrar [23] que, ao variarmos independentemente $h$ e $\omega$, i.e.: tomando $h_{t}=h+t \mu$ e $\omega_{s}=\omega+s \gamma$ onde $\mu$ é um (0,2)-tensor simétrico em $T M$ e $\gamma \in \Gamma\left(\Lambda^{1}(T M) \otimes \mathfrak{g}\right)$, obtemos, para a variação de $h$ :

$$
\left.\frac{d}{d t}\right|_{t=0} S\left(h_{t}, \omega\right)=\int_{M}\left({ }^{M} R_{i j}-\frac{1}{2}{ }^{M} R h_{i j}-T_{i j}\right) \mu^{i j} \operatorname{vol}_{M}
$$

onde $T_{i j}$ é o tensor de energia momento relativo ao campo de Yang-Mills. Claramente (4.61) resulta na equação de Einstein tendo o campo de Yang-Mills como fonte. A demonstração de (4.61) em si envolve longos e tediosos cálculos e pode ser encontrada em qualquer bom livro de relatividade geral, por exemplo [15] e [8], ou como mencionamos, em [23] de forma mais completa. A variação em relação a $\omega$, como somente o termo $\langle\Omega, \Omega\rangle$ depende de $\omega$, já foi calculada em (3.6) e fornece justamente a equação de Yang-Mills:

$$
D^{*} \Omega=0
$$

Portanto obtivemos que os campos obedecem as equações corretas para ambos a relatividade geral e campos de Yang-Mills, ou seja, obtivemos toda a dinâmica resultante das duas teorias a partir de uma métrica de fibrado em $P$. Dessa forma, é possível não só unificar as duas teorias, mas ao alcançarmos esta unificação, os campos de Yang-Mills, assim como o gravitacional, desaparecem como forças e são descritos por pura geometria, no sentido que partículas percorrem geodésicas de uma geometria riemanniana apropriada. 


\section{Bibliography}

[1] H. WEYL Gravitation and Electricity Sitzungsber. Preuss. Akad. Berlin, 1918, 465

[2] L. O'Raifeartaigh The Dawning of Gauge Theory, Princeton University Press, 1997

[3] W. Bоотнву An Introduction to Differentiable Manifolds and Riemannian Geometry, Academic Press, 2002

[4] R.P. Feynman The Feynman Lectures in Physics, Addison Wesley, 1963

[5] R. Penrose The Road to Reality, Knopf, 2004

[6] F. WARner Foundations of Differential Manifolds and Lie Groups, Spinger, 2000

[7] R. PALAIS The Geometrization of Physics, Lecture Notes, Tsing Hua University, 1981

[8] J. BAEz Gauge Fields, Knots and Gravity, World Scientific Publishing, 1994

[9] S. MoRITA The Gcomctry of Diffcrential Formus, Translations of Mathematical Monographs, AMS Press, 1997

[10] G.L. NABER Topology Geometry and Gauge Fields: Interactions, Spinger, 2000

[11] M.F. АтіYAн Geometry of Yang-Mills Fields, Scuola Normale Superiore, Pisa, 1979

[12] L.M. Sibner , R.J. Sibner, K.K. UhlembeCK Solutions of Yang-Mills equations which are not self-dual., Proc. Natl. Acad. Sci. USA 86, 8610 (1989)

[13] G. Bor Yang-Mills Fields which are not self-dual., Commun. of Math. Phys. 145, 393-410 (1992)

[14] L. D. Landau, E.M. Lifshitz The Classical Theory of Fields, Butterworth Heinemann, 1999

[15] R. WALD, General Relativity, University of Chicago Press, 1984

[16] S. Helgason, Differential Geometry, Lie Groups and Symmetric Spaces, Academic Press, 1978

[17] V. Gulllemin, A. Pollack, Differential Topology, Prentice-Hall, Englewood Cliffs, NJ., 1974. 
[18] R. Engelking , General topology, Heldermann Verlag, Berlin, 1989

[19] M.J. DufF, Kaluza-Klein Theory in Perspective, arXiv:hep-th/9410046

[20] J. Lafontaine, D. Hulin, S. Gallot , Riemannian geometry, Universitext, Springer-Verlag, Berlin, 1990

[21] F. Zheng , Complex Differential Geometry, International Press, 1989

[22] M. SpIVAK , A Comprehensive Introduction to Differential Geometry, V.2, Publish or Perish, Inc., Boston, 1970

[23] D. Bleecker , Gauge Theory and Variational Principles, Dover Publications, 2005 